\title{
Reflexivity, Internal Conversation and Societal Influences on Higher Education Students' Consideration of Values and Priorities during their Citizenship Studies
}

Linda Marie Rowan

\author{
A thesis \\ submitted to Victoria University of Wellington \\ in fulfilment of the requirements for the degree of \\ Doctor of Philosophy
}

Victoria University of Wellington

2020 



\begin{abstract}
My thesis examines the reflexive processing of knowledge, beliefs, values and personal priorities in the internal and external conversations of students during a period of university study. In higher education, learners encounter the values and views of knowledge prioritised by political, institutional, departmental and academic discourses; beliefs, values and dispositions which may differ from their own. Currently there is little understanding of how university students examine and act on new understandings of knowledge in light of their existing reference points and priorities. I use structure-agency and reflexivity theory as lenses to understand individuals' agentic responses to the personal, social and structural enablements and constraints encountered in their university studies and daily lives.
\end{abstract}

Using reflexivity methods drawn from Margaret Archer's work, I investigated students' responses to citizenship concepts presented in three compulsory courses at one Aotearoa/New Zealand university. My research involved a unique application of framework analysis methods to draw themes from the 31 participants' stories while retaining the integrity of each narrative.

In a new application of Archer's work, I found that some participants demonstrated controlled reflexivity in containing their reflexive thought processes in response to situational changes such as family trauma or mental health. Controlled reflexivity ensured the actor balanced their concerns against their projects and goals to manage and contain both their internal and external deliberations. This research challenges Archer's idea that the disruptions of late modernity removed people from their natal contexts, increasing their need for higher levels of reflexivity. While reflexivity shifts when students' values and concerns are challenged, I found that technological developments have allowed individuals to retain more and deeper connections with their natal context than in Archer's work. Furthermore, I argue that Archer's claim of a reflexive progression in dominant modes due to increased education is too simplistic and fails to acknowledge that students' reflexive practices are highly contextual (such as living in a bicultural country like Aotearoa/New Zealand) and strongly influenced by personal circumstances. Internal conversations for my research participants were complemented with external conversations to build reflexivity. Single, dual or multi modes of reflexivity were revealed in study-work life as students' personal priorities shifted. The specificity of reflexive 
processing means reflexivity typologies need to be robust to be applied across cultures and contexts.

This work is a reminder to policy developers, universities, teachers and employers that the "invisible" personal characteristics and attributes that society seeks to see in new graduates are neither easy to assess nor to confirm using typologies. Academics need to remain open to understanding the multiple intersections of the study world with individuals' wider social worlds and circumstances.

Keywords: Higher education students, reflexivity, structure-agency, internal conversation, Archer's mental activities, diverse students, citizenship 


\section{Acknowledgments}

I am grateful for and appreciative of the support I have had over the extended period of study required to complete my PhD degree. I have relished both the intellectual stimulation and the social connections I have made through this research.

In particular, I want to acknowledge the commitment and willingness of my participants to step into the world of this research - with a complete stranger. Their wisdom and generosity continues to amaze me. I admire their dedication and determination to complete their studies despite complex lives and the curveballs that came their way. The bounty of their lives and thoughts provides the layers of richness in this research.

My supervisors - Dr Kathryn Sutherland, Centre for Academic Development, and Dr Stephanie Doyle, School of Education, of Victoria University of Wellington - are the greatest! They have challenged my thinking, passed on their given insights, kept me on the hop, and provided the right level of encouragement, advice, and support that I needed over the past five years. I know I sometimes left them baffled, but the breadth and depth of their knowledge and experience has been an invaluable resource. Thank you.

Thank you, Linda P. and Philippa B. for your eagle eyes and support.

My own priorities and values remain family and people centred. My family - Daryl, Stefan and Dani (and Luna), and Errin - have been my amazing, quiet, and steadfast support team. Their humour, encouragement and belief that I could finish this marathon kept me going and smoothed the journey. I do not expect them to read the whole thesis, but they know I owe them heaps - for those moments that have been put on hold which would usually create our family memories. They will not let me forget that. Now, it is time for us to catch up and create more moments. 


\section{Table of Contents}

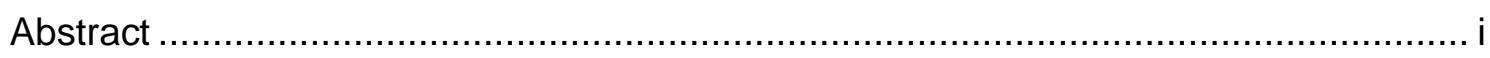

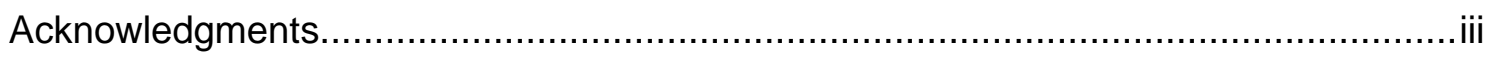

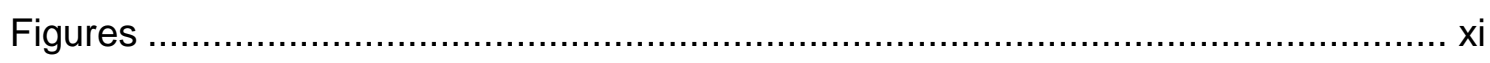

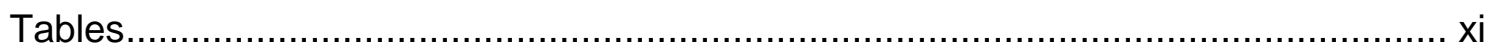

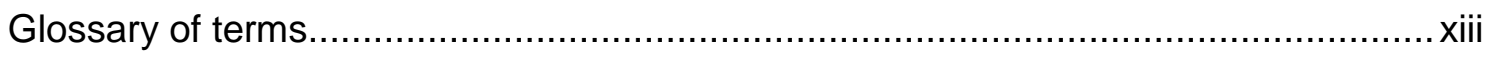

Chapter 1. Introduction, context and brief literature review ................................ 1

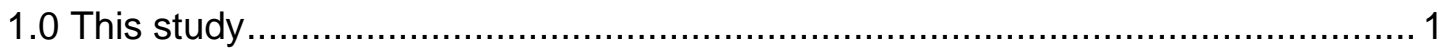

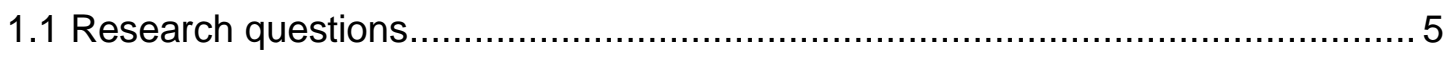

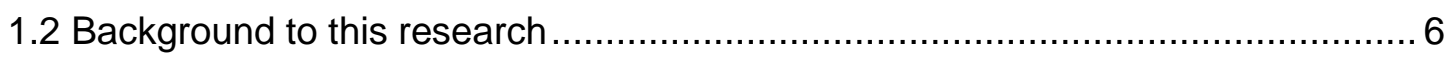

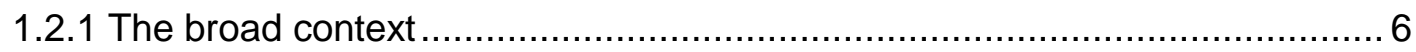

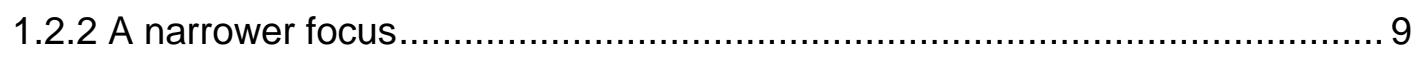

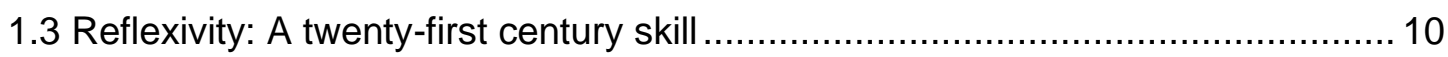

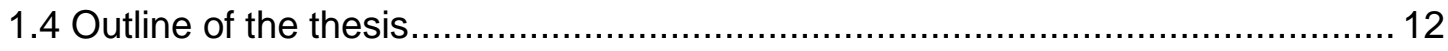

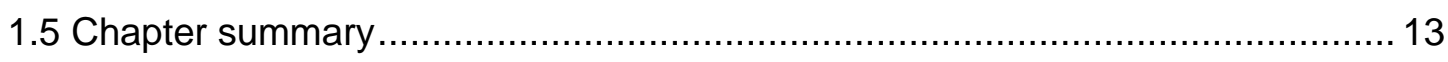

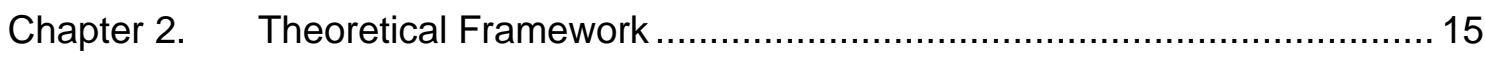

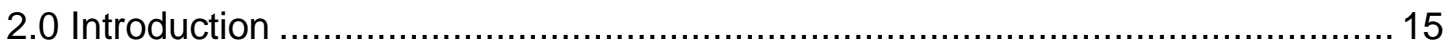

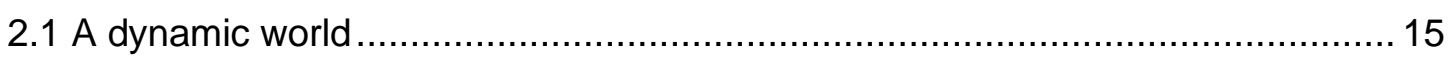

2.2 Critical realism, structures, agents, agency and reflexivity ............................. 20

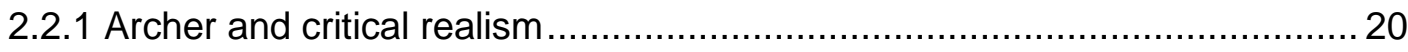

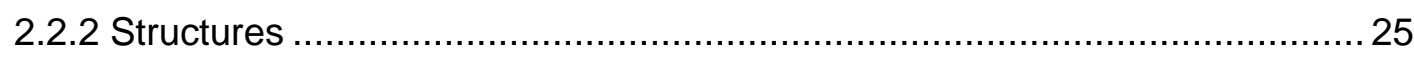

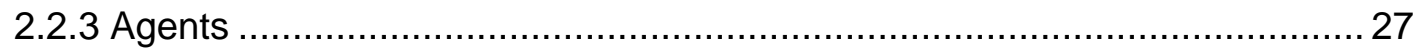

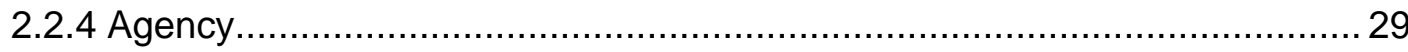

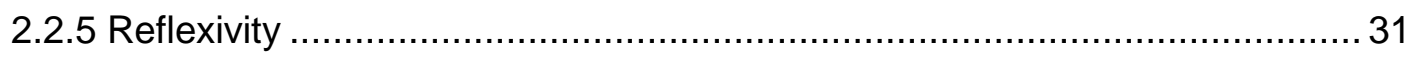

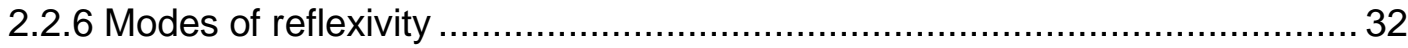

2.3 Internal conversation and determining internal conversation .......................... 37 


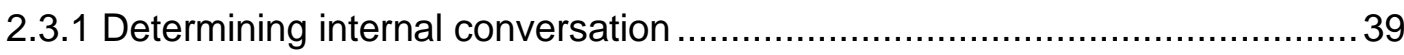

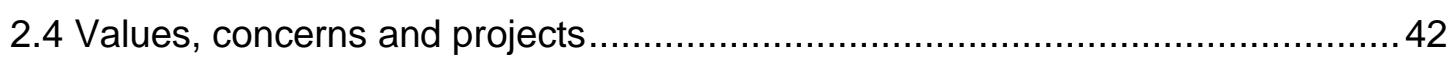

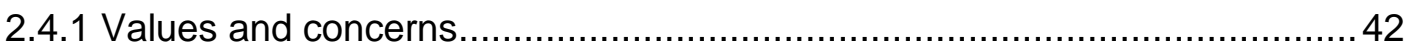

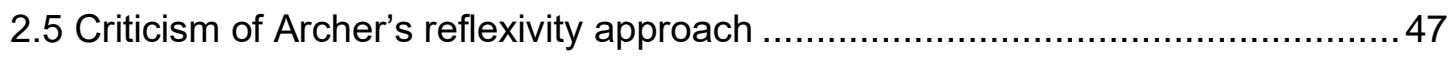

2.5.1 Archer's reflexivity - criticisms and responses .................................. 47

2.5.2 Empirical studies using Archer's reflexivity approach ............................51

2.5.3 Adaptations to Archer's reflexivity and internal conversation .....................53

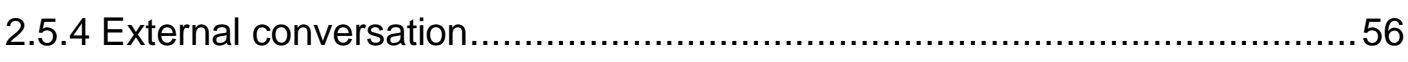

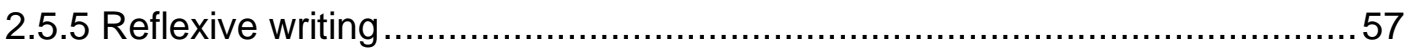

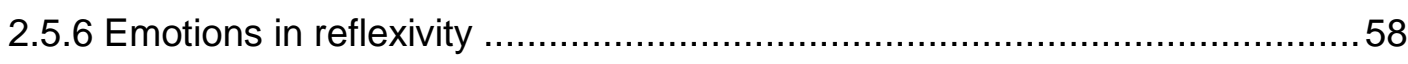

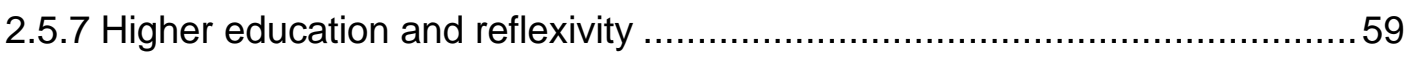

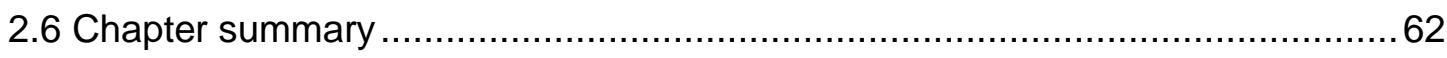

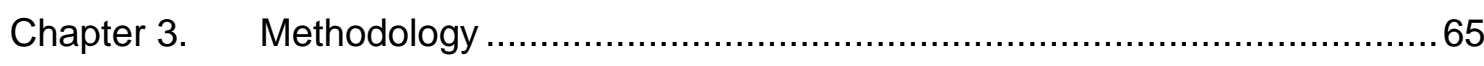

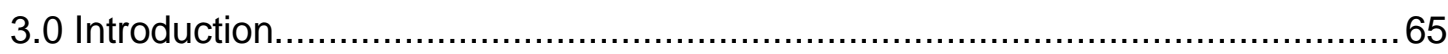

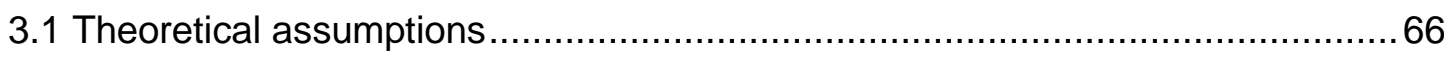

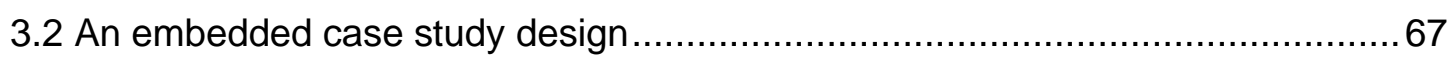

3.3 Qualitative longitudinal research (QLR) methodology .................................69

3.4 Manaakitanga - culturally and socially responsible research ..........................71

3.5 Research processes and data collection .............................................. 72

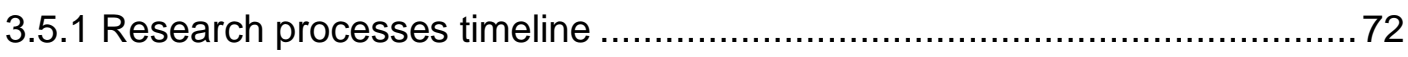

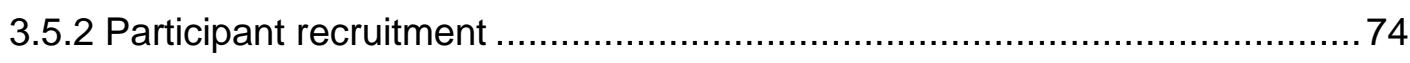

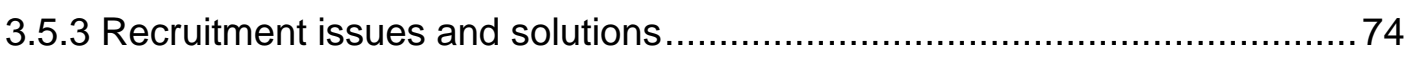

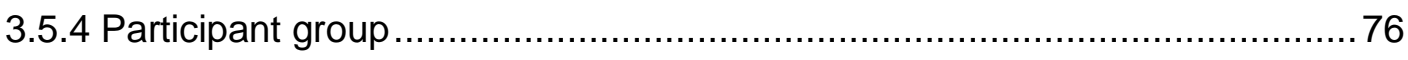

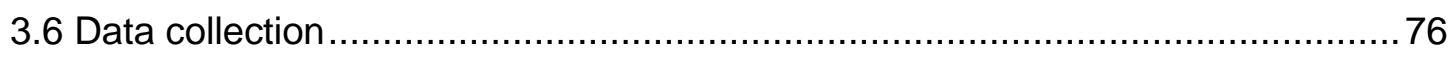

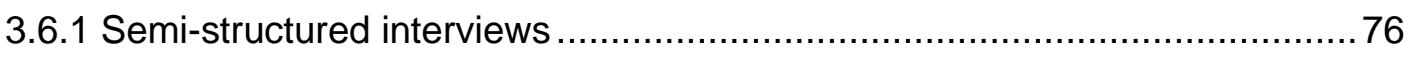

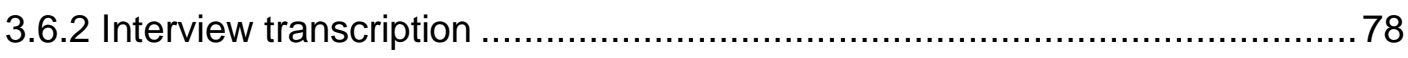

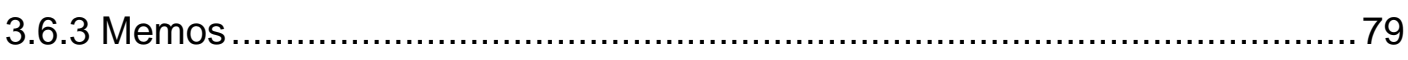

3.6.4 Reflective writings and discussion forum posts ................................... 79

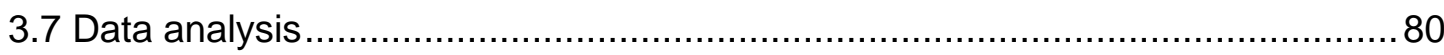




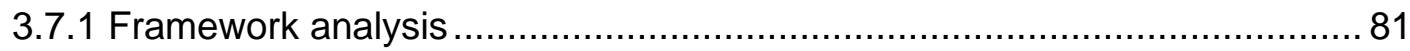

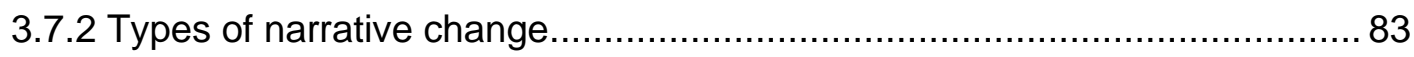

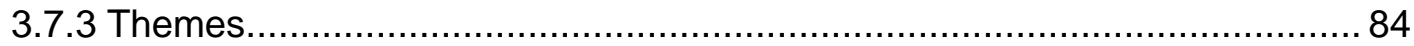

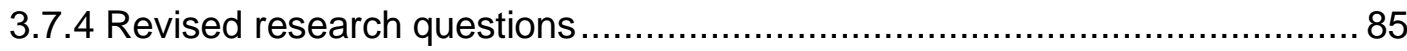

3.8 A note on the codes used to identify participant quotes ................................. 85

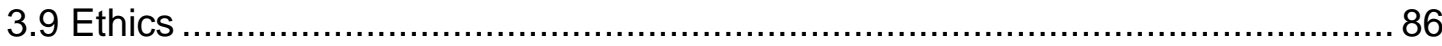

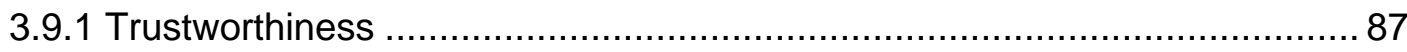

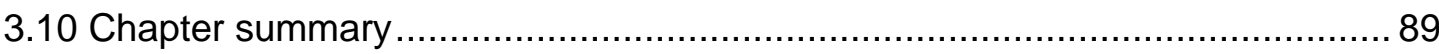

Chapter 4. Findings from framework analysis............................................... 91

4.1 Participants' contexts and commitments.................................................... 91

4.2 Citizenship as an external reflexive conversation .......................................... 93

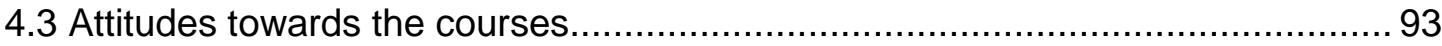

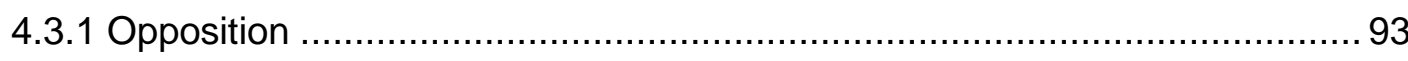

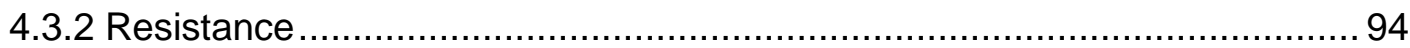

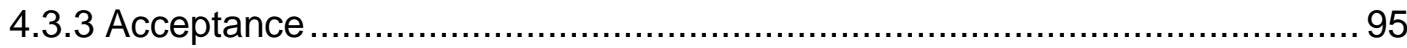

4.4 Reference points for processing citizenship knowledge ................................ 95

4.4.1 Personal identity and citizenship - 100-level ..........................................96

4.4.2 Global citizenship and responsibilities - 200-level ..................................98

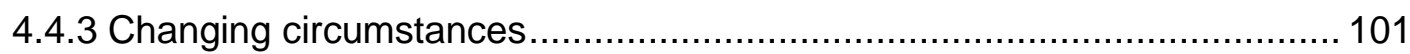

4.4.4 Active citizenship - 300-level ......................................................... 102

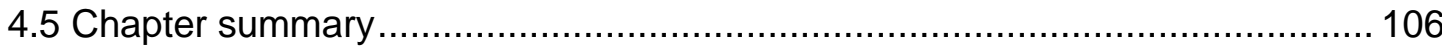

Chapter 5. Participant vignettes and analysis ......................................... 107

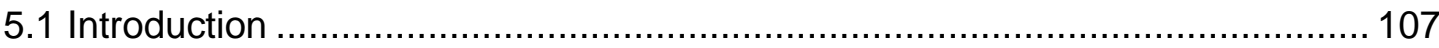

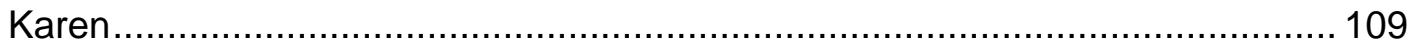

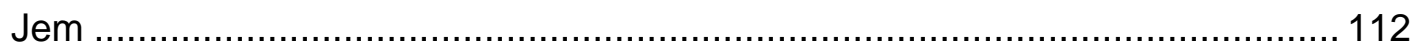

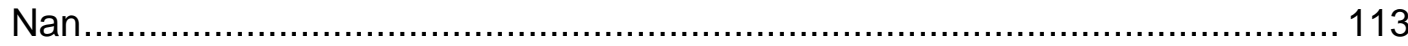

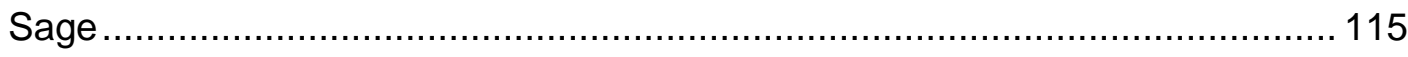

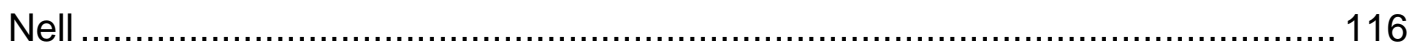

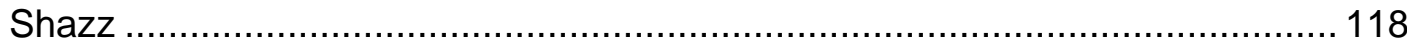


Elsie

Kane 121

Deedee 122

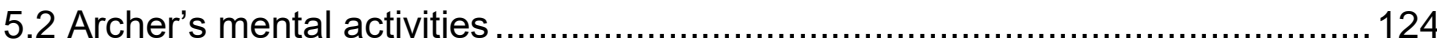

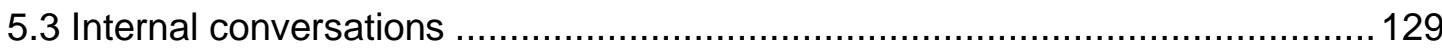

5.3.1 High-on-going-use of internal conversation ........................................ 130

5.3.2 Episodic-for-a-purpose internal conversation ..................................... 133

5.3.3 Low or restrained use of internal conversation. ...................................... 134

5.4 Comparison with Archer's modes of reflexivity.......................................... 135

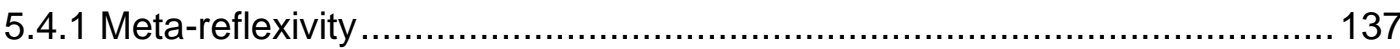

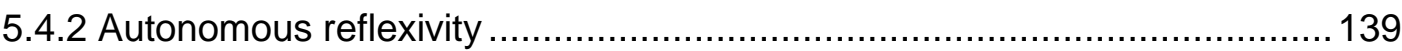

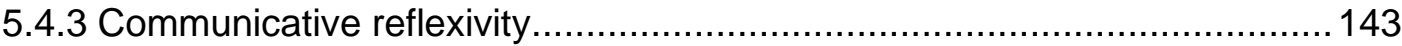

5.4.4 Fractured reflexivity moves to communicative reflexivity ........................ 146

5.4.5 Fractured reflexivity and meta-reflexivity ........................................ 147

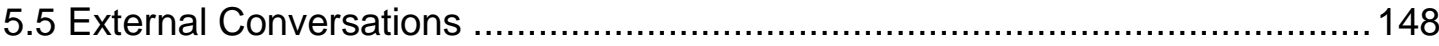

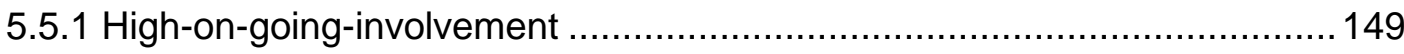

5.5.2 Episodic-for-a-purpose ................................................................. 151

5.6 Course assessment as written external conversation ................................. 152

5.7 Discussion forums as written external conversation.................................... 153

5.8 Social media as written external conversation ........................................... 155

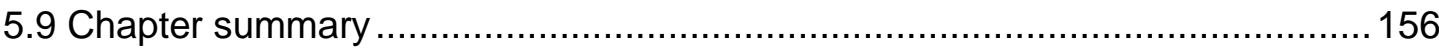

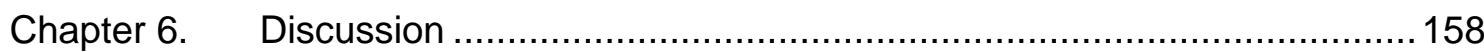

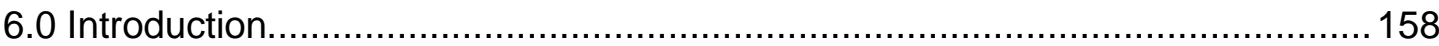

6.1 Reflexivity and contextual continuity and discontinuity ............................... 160

6.1.1 On-going contextual discontinuity and agency ..................................... 165

6.1.2 Changes in circumstances and modus vivendi ..................................... 166

6.2 Internal conversation and social interactions ............................................. 167

6.2.1 Individual or communal needs and concerns....................................... 168

6.2.2 Prioritising others' needs first ......................................................... 169 


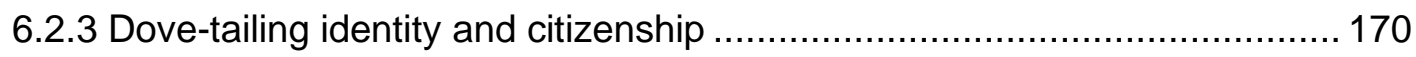

6.2.4 Confluences in personal reflexivity and citizenship............................... 172

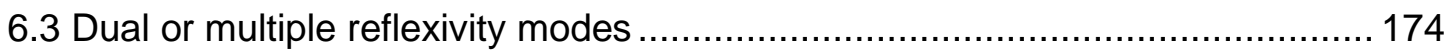

6.3.1 Cross-checking reflexivity modes................................................... 177

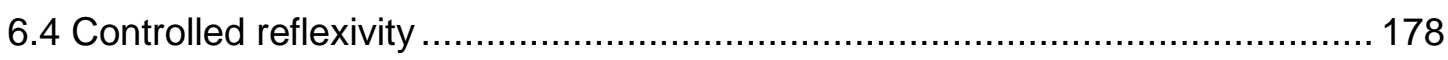

6.5 Writing extends the internal conversation ................................................ 179

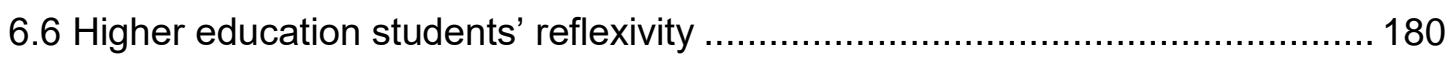

6.6.1 Structure-agency in individual reality.......................................... 181

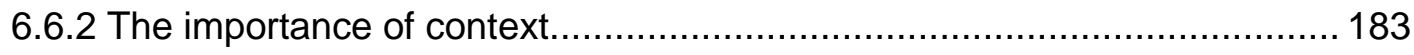

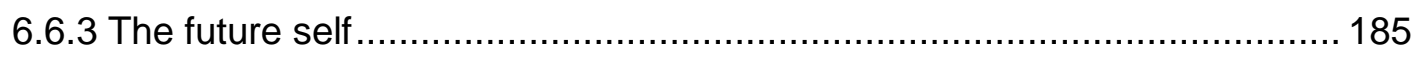

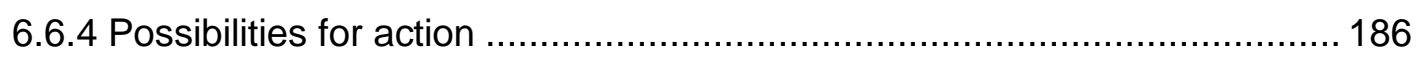

6.6.5 Project choice and implementation .............................................. 189

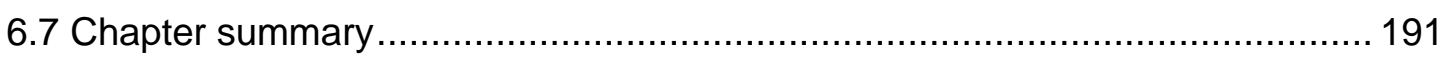

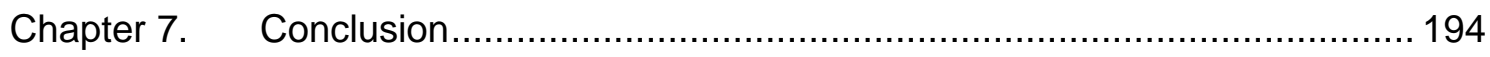

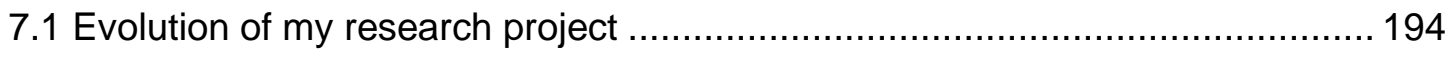

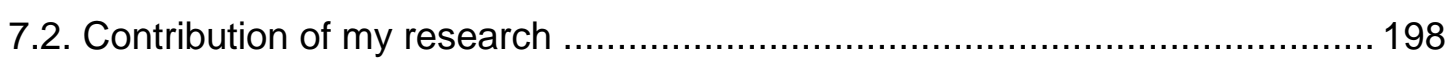

7.2.1 Reflexivity and internal conversation theory ......................................... 198

7.2.2 Higher education students' development of invisible skills ....................... 200

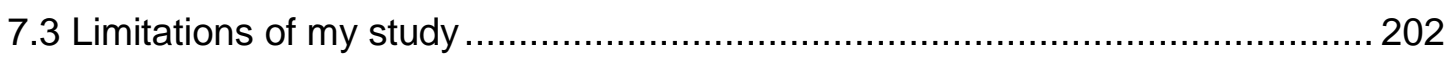

7.4 Future directions for research.............................................................. 203

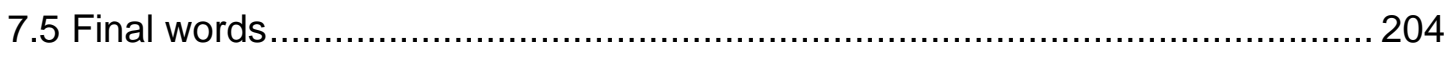

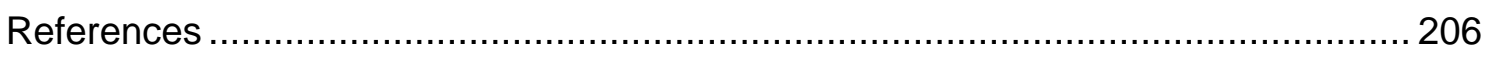

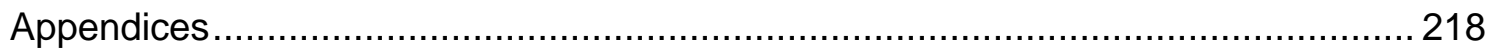

Appendix A: Recruitment phases and recruitment outcomes............................ 220

Appendix B: Participant contexts and change over time .................................. 222

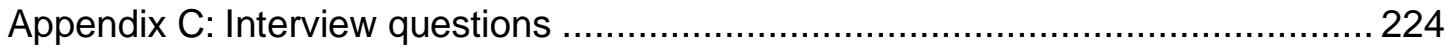

Appendix D: Additional questions for interviews: Archer's 10 mental activities of

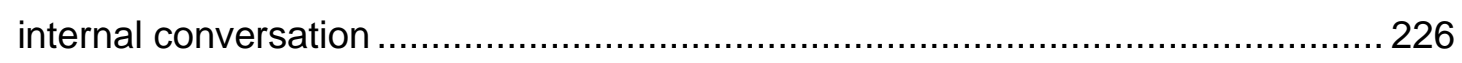

Appendix E: Framework analysis worked chart: Elsie...................................... 228 


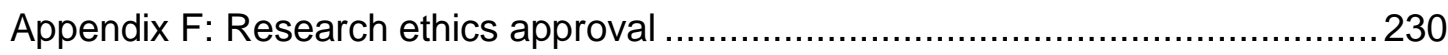

Appendix G: Memo prepared from interview one transcript for interview two ......... 232 


\section{Figures}

Figure 3.1 Timeline and overall design of the study 73

Figure 3.2 Framework data analysis charts for this study (based on Lewis, 2007) ...... 82

\section{Tables}

Table 2.1 Primary concerns of University of Warwick students (adapted from Archer, 2012)

Table 3.1 Analytical themes generated from participant data. 84

Table 3.2 Identification codes used with quotes 86

Table 4.1 Participant age, work-study status and ethnicity 92

Table 5.1 Participant completion and interview stages. 109

Table 5.2 Summarised from Archer's (2007) list of mental activities 125

Table 5.3 Participants use of internal conversation and Archer's mental activities 127

Table 5.4 Frequency, focus and use of internal conversation 130

Table 5.5 Participant alignment with Archer's modes of reflexivity (2007). 136

Table 5.6 Frequency, focus and use of oral external conversation. 148 


\section{Glossary of terms}

Aotearoa - Māori name for New Zealand.

Internal conversation - an internalised personal reflexive practice where an individual considers in a questioning, speculative manner the interplay between their environment and themselves. It is interior, subjective, and reasoned. Synonyms: inner deliberation, inner dialogue, inner conversation, inner deliberation, internalised considerations, contemplation, to consider inside one's head dialogue (Archer, 2003, 2007).

Koha - (Māori) gift, present, offering, donation, contribution - especially one maintaining social relationships and has connotations of reciprocity (Moorfield, 2019).

Kōtahitanga - (Māori) unity, togetherness, solidarity, collective action (Moorfield, 2019).

Kaitiakitanga - (Māori) guardianship, stewardship, trusteeship, trustee. To care for the environment and its people (Moorfield, 2019).

Manaakitanga - (Māori) hospitality, kindness, generosity, support - the process of showing respect, generosity and care for others, their cultures and values (Moorfield, 2019).

Māori - (Māori) indigenous New Zealander, indigenous person of Aotearoa/New Zealand.

Modus vivendi - a set of practices which represents and privileges the matters which are important to the individual in order to create a way of life (Archer, 2007, p 148).

Mokopuna - (Māori) descendant, grandchildren, grandchild - child or grandchild of a son, daughter, nephew, niece, etc (Moorfield, 2019).

Morphogenesis - "those processes which tend to elaborate or change a system's given form, structure or state" (Archer, 2012, p. 50.).

Morphogenetic approach - Archer's explanatory means for examining the structureagency interplay and their outcomes.

Morphostasis - those processes in a complex system that tend to preserve the form, structure or state of the system (Archer, 2012). 
Natal context - traditional or childhood background or location.

Pākehā - (Māori) New Zealander of European descent - originally applied to Englishspeaking Europeans living in Aotearoa/New Zealand (Moorfield, 2019).

Reflexivity - "the regular exercise of the mental ability, shared by all normal people, to consider themselves in relation to their social contexts and vice versa" (Archer, 2012, p. 1).

Tauiwi - (Māori) foreigner, European, non-Māori, colonist (Moorfield, 2019).

Tangata whenua - (Māori) local people, hosts, indigenous people - people born of the whenua (land), i.e. where the people's ancestors have lived and where their placenta are buried. (Moorfield, 2019).

Te Reo - Māori language

Tūrangawaewae - (Māori) standing, place where one has the right to stand - place where one has rights of residence and belonging through kinship and whakapapa (Moorfield, 2019).

Whakamana - (Māori) to give authority to, give prestige to, confirm, enable, authorise, legitimise, to empower (Moorfield, 2019).

Whakamaranga - (Māori) to uphold, lift up, esteem (Moorfield, 2019).

Whakapapa - genealogy, to recite genealogies in proper order (Moorfield, 2019). 


\section{Chapter 1. Introduction, context and brief literature review}

\subsection{This study}

My thesis looks at how social changes in reflexive understanding take place in individuals through participation in three citizenship courses at university. Students bring their own sets of beliefs and the embedded values of their social networks when studying in higher education (Harland \& Pickering, 2011; Mayhew, Wolniak, Rockenbach, Bowman, \& Seifert, 2016). They have complex work-study-life contexts and understandings of their personal situations. They are not blank sheets; there are social relationships and networks which are as important, or more important, to them than their studies. A period of study in university is considered an opportunity for learners to build their professional understandings (Ashwin, 2014), their social identity (Kaufman, 2014) and their workplace skills (Ahier, Beck, \& Moore, 2003; Ministry of Education, 2014; UNESCO World Conference on Education, 1998). Yet, in higher education learners encounter the knowledge, values and attitudes prioritised by political, institutional, departmental and academic discourses; beliefs and values which may differ substantively from their own. These values are encapsulated in the attributes and capabilities described in graduate profiles as indicators of students' work-readiness (Barrie, 2006; Kensington-Miller, Knewstubb, Longley, \& Gilbert, 2018; Spronken-Smith et al., 2013). What policy makers, institutional leaders, teachers and students do not understand is how learners work through the values and beliefs presented during their university studies, in their discipline knowledge and associated social exchanges, and how students consider these ideas to make decisions about their place in their thinking and actions.

The thesis considers the relationship between individuals and society in how decisions on citizenship beliefs, values and concerns are considered and made. Accepted knowledge constitutes a set of ideas or beliefs consistently held within society to be true. Values based on these beliefs indicate the worth the accepted knowledge has for the group (Berger \& Pullberg, 1965) and form society's structures (Archer, 2003; Berger \& Luckmann, 1966). Attitudes towards the things valued by social groups affects behaviours and actions (Buissink-Smith, Mann, \& Shephard, 2011). While attitudes 
towards social values and knowledge values may shift, beliefs have a more permanent nature. At the individual level, values and goals, or priorities, are also shaped by personal concerns and contexts as a part of wider socialisation processes (Archer, 2003; Berger \& Luckmann, 1966). Thus, the values and priorities a person holds form the landscape of the individual's reality, inform their identity and influence their actions. When a person encounters new ideas, they consider this knowledge in light of their current reality to determine if and how they will act on it in relation to their current values and understandings. The freedom or ability to act is an indicator of an individual's agency (Archer, 2000, 2003).

My research project followed a cohort of students through three levels of undergraduate study in core citizenship courses to look at how shifts in their reflexive thinking occurred, the reference points they used in their decision-making and subsequent actions. The course themes and topics were intentionally provocative, intending to challenge current thinking. I used critical realist Margaret Archer's theories of morphogenesis, reflexivity and internal conversation (Archer, 2003, 2007) to understand how individuals made decisions about their beliefs and concerns within the wider social world. Archer argues that, as individual agents, people hold powers and characteristics that set them apart from the powers and properties of society (Archer, 2003). She believes if researchers separate the individual from the structural world it is possible to look at the influences of social structures on people's agency, through understanding the structural enablements and constraints (Archer, 2003).

There has been considerable debate and discussion about Archer's structure-agency theory and its place in social theory (see Elder-Vass, 2007; Farrugia \& Woodman, 2015; King, 2010 for example). In particular, arguments have centred on the individualisation of the person in separating out and treating agents as detached from societal structures and their resources (Farrugia \& Woodman, 2015; Mutch, 2004) and the downplaying of social interactions (Burkitt, 2016; Caetano, 2015b) - and individualisation. The structureagency debate continues and remains unresolved. However, less consideration has been given to Archer's reflexivity methodology and methods for determining internal conversations.

My qualitative longitudinal research design extends applications of Archer's methods to consider the effects of learning and social interaction on the lives and values of distance learning university students who are actively engaged within multiple study, social and work environments. The novel use of the framework analysis matrix system (Lewis, 2007; J. Smith \& Firth, 2011) enabled the exploration of thematic changes in individual's 
thinking on citizenship within their dynamic social contexts. The findings remind policy makers, universities and teachers of the complexity of teaching and assessment of the development of affective graduate attributes in a changing world.

In my study, I make the assumption that there is a difference between the influences of compulsory or formal education and adult or higher education environments on personal understandings and attitudes based on their social circumstances. In formal education, learning is believed to play a role in individuals achieving agency (Biesta \& Tedder, 2007). This view sees agency as something that is acquired or generated through participation in learning activities, rather than a personal characteristic that is developed over time. In contrast, higher education is a learning context which acknowledges the individual as an agent and provides opportunities for cognition, as agents intellectually and socially engage as students. A societal belief is that over a student's time at university various institutional, moral and ethical values are accumulated to positively shape a person's democratic and personal values through their interactions with academics and their peers (Ahier et al., 2003; Biesta, Bouverne-De Bie, \& Wildemeersch, 2014; Kuh \& Bridges, 2010). Yet little research has been conducted on how these values are considered by students against their existing values and beliefs. Research on student learning has predominantly focused on engagement within traditional face-to-face study environments and students cognitive and behavioural development (Haggis, 2009; Mayhew et al., 2016). While there has been a recent shift to consider the socio-cultural contexts of learning through student experience and engagement lenses (e.g., Kahu, 2013), little research attempts to bridge the gap in understanding of a student's agency, their wider life and the values presented in study.

In addition, I believe that in the globalised world, higher education cohorts are becoming more diverse and reflect changes in social cohesion. In addressing political, social equity and economic goals, universities have developed policies and strategies which open their doors to wider participation from within their own communities and other countries (Biesta \& Simons, 2009). As a consequence, the characteristics that distinguished between traditional and non-traditional students are blurring (Kahu, Stephens, Zepke, \& Leach, 2014; McInnis, 2004; Pascarella \& Terenzini, 2005). Full-time or part-time enrolment status obscures the fact that many students will be working and studying at the same time (Poskitt, Rees, \& Suddaby, 2011). Consequently, the higher education experience is no longer confined to a one-off event for traditional student groups. People return to study through-out their lives to up-grade their qualifications, supplement professional development or engage with current knowledge (Kahu et al., 2014). 
Distance students have values, outlooks and behaviours that more align with the society in which they live and work, not just the university they attend. In addition to education being a primary external influence on student lives, wider technological developments have made other social networks and their influences accessible.

Higher education values the development of new knowledge and critical thinking. It could be expected a period of study at university would encourage the reconsideration of personal and societal values and goals. However, in a review of higher education research conducted between 2000 to 2015, Mayhew et al. (2016) found the main investigation of values had been on socio-political, civic and community values, or religious or spiritual attitudes. The research evidence from predominantly northern hemisphere, western higher education institutions suggested that the university or college structure and culture had little influence in bringing about changes in students' attitudes towards racial or ethnic matters. Likewise, reports on higher education's effects on the development of civic values were conflicted. Attitudes towards racial attitudes and ethnic and cultural diversity were indirectly, but positively, influenced by students mixing with other students (Mayhew et al., 2016). However, little research considers how beliefs are challenged and reflexive commitments changed through these social interactions. My research project begins to address this gap in understanding.

To understand the wider contexts of social interactions on university students' reflexivity, I wanted to explore the structural and social influences that contour students' life values and concerns. Archer $(2007,2012)$ applied her theory of reflexivity and internal conversation to describe four reflexive modes that are used by people in considering their values and goals towards social change. Her methodological approach and tools provide a means to look at the structure-agency interactions, as new understandings or circumstances are discerned and possibilities for responding to these are considered. As each person's reality is both socially located and each view is individualised (Berger \& Kellner, 1981; Cousin, 2009; Denzin \& Lincoln, 2000), tapping into students' thinking about their social realities in their internal conversations would help to understand their reflexive considerations of their course knowledge and their own values.

To do so, I also needed to consider how the configurations of students' social contexts, norms and interactions were operating (Crotty, 1998) over the period of their study. I used qualitative longitudinal research methodology, narrative approaches and adaptations on Archer's internal conversation methods to collect the stories of a group of diverse higher education students participating in three citizenship courses core to their undergraduate degree. As a sole researcher, I used framework analysis procedures 
in a novel manner to feature each participant's overall story while looking for themes and variations in thinking within levels of the citizenship courses and across their studies.

\subsection{Research questions}

The key question I wanted to find answers to was - how do diverse higher education students reflexively process and act on value-laden knowledge presented within their studies?

To answer this complex question required a finer understanding of the knowledge, values and concerns that are important in each individual's identity and their reflexive processing, and the place their social interactions have in considering new ideas. Recent developments in reflexivity studies exposed a foundational debate over the place of external and written dialogues in reflexive deliberations (Caetano, 2017b; Chalari, 2007). I wanted to incorporate both external and written aspects, alongside the internal conversations because university students are called to participate in discussions and generate self-reflective documents as evidence of their academic development. Finally, in considering these various configurations of and influences on reflexivity, I wanted to find out how much agency individuals felt they had to act out their reflexive processing of the knowledge they had encountered in their university studies, in light of their personal values. These exploratory refinements formed the sub-questions to answer the investigative question.

The sub-questions were:

- What knowledge, values and concerns are important in individuals' reflexive processing during their citizenship courses?

- What social interactions are important in considering new ideas from the citizenship courses?

- How do these internal and external conversations work?

- How much agency do individuals feel they have to act out their reflexive processing?

In the next section, I present a description of the higher educational panorama and a brief sketch of my place as researcher in this study. 


\subsection{Background to this research}

\subsubsection{The broad context}

The research presented in this thesis is situated within the field of higher education, and within a dynamic post-compulsory, higher education system. Multiple layers of political, social and academic conditions influence participation and teaching in higher education at the macro-level and, ultimately, how society benefits from individuals' educational development. In the postmodern world, massification, globalisation, internationalisation and widening access to education have changed the landscape for universities, academics and students (Ahier et al., 2003; Jarvis, 2007). The changing national and global economy and demand for highly skilled and educated employees, has seen the focus for higher education shift imparting knowledge to preparing graduates who can meet the expectations of the twenty-first century global environment.

Higher education has become a space not only for intellectual development, but also for the advancement of political goals. Goals include economic development, social change, social justice and the empowerment of specific groups, and democratic or nationalistic education in an increasingly connected world (Colby, Ehrlich, \& Beaumont, 2003; Rhoads \& Szelenyi, 2011; G. Smith, Ottewill, Jubb, Sperling, \& Wyman, 2008; Veugelers, de Groot, Llomovatte, \& Naidorf, 2017). For over two decades western universities have prioritised meeting employer demands for a highly educated and skilled workforce. While the focus has been on growing national economies, other significant global shifts in the social world (e.g., mass movements of people) and technological world (e.g., availability of knowledge and media communication platforms) have had significant impacts on national demographics and social systems. The increased diversity of people and cultures both within and between nation-states, challenges to current economic and democratic systems from within and beyond national borders, and declining participation in civic processes (Biesta \& Simons, 2009; Klemenčič, 2018; UNESCO World Conference on Education, 1998) has led to calls for universities to also address these changes. Institutional policies, approaches and practices have adapted to meet these political priorities; universities have taken on the role of 'fixers', geared towards improving individuals or systems (Case, 2013). Improvements include providing opportunities for students to develop democratic citizenship attitudes and their associated responsibilities.

As a result, academic departments have incorporated citizenship skills and capabilities into the graduate profiles used to assess learning (Ahier et al., 2003; Arthur \& Bohlin, 2004; Case, 2013). Adult educationalists Usher, Bryant, and Johnston (1997) argued 
where educating for citizenship had once just been associated with development of the knowledge, skills and attitudes which allowed citizens to meaningfully participate in democratic society, in postmodernism citizenship education has become tied to wider social, economic and cultural rights and values. University graduate profiles now also prioritise employer values as well as discipline and pedagogic knowledge.

Student development is assessed against agreed profiles of desired graduate outcomes - skills, knowledge and characteristics (Barrie, 2006; Spronken-Smith et al., 2013). Graduate outcomes are value propositions and include the development of affective attributes of learning which are difficult to assess (Buissink-Smith et al., 2011; Kensington-Miller et al., 2018). Any set of values is specific to the person or group who holds them, open to interpretation by others, not always obvious to the self or others, and subject to change (Harland \& Pickering, 2011). It can be expected, then, that the values and characteristics presented in graduate profiles, their associated courses, and by course teachers may differ from those of the diverse student cohort currently studying in higher education. How students contend with the differences between their own values and concerns and those presented in their university courses or by their peers is unknown, and the focus of this study.

My study draws from sociology to frame the interactions between higher education students and the social structures at play in students' lives as they consider value-laden concepts during a course of citizenship study. Societal views of knowledge, the structures, beliefs and goals of higher education underpin academics' teaching philosophies and practices, and concepts of learning (Giddens \& Sutton, 2014; Jarvis, 2007). These beliefs affect what is taught, how it is taught and what and how it is assessed (Harland \& Pickering, 2011). They affect how students are viewed - whether as objects, consumers, agents or actors - and consequently, the role students have in learning and constructing knowledge (Felten et al., 2019; Williams, 2012). Societal views affect beliefs about students' wider social circumstances and the interaction between personal and study life (Kahu et al., 2014; Weidman, DeAngelo, \& Bethea, 2014). In turn, the confluence between personal and study life influences learner engagement and outcomes, their fit to graduate profiles, opportunity for employment, and contribution to civic and social life.

As the twenty-first century student demographic profile continues to change, students of all backgrounds and ages will continue to bring the complexities of their cultural, economic and social contexts, beliefs, work experiences and educational goals into the learning space. In September 2017, global participation rates in higher education were 
predicted to rise from $14 \%$ to $25 \%$ by 2025, and above $40 \%$ by 2100 (Cuthbert, 2017). The diversity within the student population, now, forms the 'new majority' of learners (Felten \& Lambert, In press). As Felten and Lambert note, the 'traditional' student, who was once a white, middle class, young male is no longer the majority in USA universities. There, $40 \%$ of university and four-year college undergraduate students are over twentyfour years of age, $55 \%$ are women, $50 \%$ live at home, only one-fifth of attendees were first-in-the-family in higher education (Felten \& Lambert, In press). The statistics are similar in the southern hemisphere, with $43 \%$ of students over twenty-four years of age in Australia and $50 \%$ in Aotearoa/New Zealand universities. Women outnumber men in both Australia (56\%) and Aotearoa/New Zealand (62\%). Extramural or distance learning is similar at $16 \%$ (Australia) and $18 \%$ (Aotearoa/New Zealand) (Australian Government Ministry of Education, 2018; Ministry of Education, 2018). As well as students being more diverse in terms of age, ethnicity and gender, they are also more likely to engage in their studies in diverse ways. For example, rather than higher education being a 'one-off' event in learners' lives, it has become an on-going part of the development of skills for employment, social engagement, personal agency and lifelong learning, and is undertaken at a variety of stages in people's lives.

On-going discussions within higher education have called for more consideration of these diverse students' situations, their place in higher education and their development of agency in the teaching and learning relationship. Inclusive academic approaches (such as staff-student partnerships and building academic success in minority groups) work towards understanding learner agency (Ashwin, 2008; Baker, 2019; Case, 2015). Yet these approaches continue to take a western view of students as individualistic, autonomous agents rather than socially connected, relational, interdependent people who are responsive to others and the situations around them (Biesta \& Tedder, 2007; Burkitt, 2016). Little is known about the relationship between social agency and learning in higher education (Biesta \& Simons, 2009). What is needed are approaches which incorporate consideration of the effects of wider social and relational conditions influencing knowledge growth and personal development.

This 'new majority' reflects the undergraduate and postgraduate university students I encounter in my teaching in Aotearoa/New Zealand. They already have work experiences, life histories and study experiences that often surpass those of the tutors and lecturers they encounter in university. They have firm values, priorities and goals and little time for tasks that do not challenge their thinking. Yet, as teachers, we know little about the wider structural, cultural and social factors that confront these students as 
agents in their daily study. We do not know how macro-, meso- and micro-level structures and temporal changes affect their personal agency - challenging or reinforcing identities and beliefs. Nor do we know how these structures and changes help, or restrict, these students to position themselves to act on their new knowledge and values. To understand how individuals' social contexts and their concerns are changed by participation in higher education, teachers need greater understanding of how structural influences affect people's choices (Clegg, 2005).

\subsubsection{A narrower focus}

Aotearoa/New Zealand university cohorts consist of domestic and international students, internal students on local campuses, and distance learning students spread around the country and the globe. Students of all ages, with different cultural and educational backgrounds, existing occupations, experiences and commitments study in full- or parttime modes (Ministry of Education, 2018).

In $2017,18 \%$ of all students in post-compulsory education were studying by distance in Aotearoa/New Zealand, with the number rising to 21.9\% in 2018 (Ministry of Education, 2018). Of these distance students, $54 \%$ ethnically identified as Pākehā/European, $19 \%$ Māori, 8.5\% Pacific Peoples, 24.5\% Asian and 5.8\% other ethnicities. More women $(62 \%)$ were in study than men (42.4\%) (Ministry of Education, 2018). In a 2011 study of Massey University distance learning students' characteristics, $66.6 \%$ of students spent more than one hour per week caring for dependents compared with $36.8 \%$ for oncampus students. Sixty-three percent were in paid employment for more than 17.5 hours a week compared with $57 \%$ of campus-based learners who averaged 12.9 work hours. Over $17 \%$ of distance students worked full-time or over 30 hours per week (Poskitt et al., 2011). They reflect the new student majority who are likely to enrol in study in higher education for the first time, or return to retrain, upgrade skills or study for pleasure, as the nature of employment and leisure changes.

My interest lies in how students from such diverse backgrounds navigate their way through value-laden academic ideas encountered within the social interactions of learning, in light of their own beliefs or concerns. As a first-in-the-family student at university myself, and a higher education teacher for the past two decades, I am conscious of the tensions and complexities of merging societal and individual beliefs and goals with those of higher education.

In 2014-5, I was a member of an academic team planning a new citizenship curriculum to help Bachelor of Arts students develop the skills, capabilities and characteristics of 
twenty-first century work-ready graduates (Massey University, 2014). Five core courses, focused on developing academic and public citizenship skills and capabilities, were incorporated into the degree in 2017 . Three interdisciplinary citizenship courses focus on exploring diverse and opposing disciplinary concepts of personal citizenship identity (at 100- or first-year level); the encounters and relationships between local, national and global communities (at 200-or second-year level); and what it means to be active citizens (at 300- or third-year level) (Kahu \& Gerrard, 2018). Teaching for the development of citizenship capabilities is an intervention, an intentional action towards a particular goal, challenging each student's understanding of themselves, their values and their concepts of citizenship.

The citizenship courses are set within Aotearoa/New Zealand, a bicultural country where citizens are ethnically identified as indigenous Māori, Pākehā/non-Māori of European decent, and Pacific Peoples, Asian and other ethnic groups (Ministry of Social Development, 2016). Learners' mixed characteristics, and social and economic demands influence their values, time and management of priorities.

Recognising the diverse pool of learners in the courses, I wanted to understand how Aotearoa/New Zealand higher education learners worked though the concepts, values and knowledge presented in the three citizenship courses in light of their own realities. Although restricted access to detailed course materials limited an investigation of the relationship between citizenship constructs and learner responses, I still wanted to know if learners felt they had agency to act on their concerns. To understand this, I turned to the concept of reflexivity.

\subsection{Reflexivity: A twenty-first century skill}

The break-down of the traditional industrial norms and social systems is considered part of reflexive modernisation and a movement towards more fluid ways of being and operating (Beck, Giddens, \& Lash, 1994). Postmodern sociological debates have shifted from looking at the powers of structures over people to look at the relationship between structures and agency. Of particular concern was how free individuals were to decide on their own actions and how much they were constrained or enabled by their social circumstances. Anthony Giddens proposed that to move into the new globalised society people would need reflexive skills to consider the choices available to them (Giddens, 1991). Critical realist Margaret Archer felt reflexive modernisation theories now placed too much emphasis on socialisation, elevating the powers of the individual over structures. Doing so obscured the emergent personal powers of the individual and how 
they might act as free agents (Archer, 2000). Instead she proposed a morphogenic system based on reflexivity to bring balance to the structure-agency debate and enable the understanding of decision-making in the individual-social interaction. In reflexive deliberation, the individual recognises the enablements and constraints faced and what they are able to do in pushing back against structures (Archer, 2003). How free they feel to act on this thinking is an indication of their agency or agential power.

Archer defines reflexivity as "the regular exercise of the mental ability, shared by all normal people, to consider themselves in relation to their social contexts and vice versa" (Archer, 2012, p. 1). Reflexivity is needed to be able to choose how to act in the absence of traditional guidelines or habitus (Bourdieu, 1984). Reflexivity occurs as an internal conversation and therefore is difficult for the researcher to observe.

In internal conversation, concerns are formed as a part of personal identity and consideration of the kind of life the individual wants to live. Reflexivity helps a person to evaluate their "social contexts in light of [their] concerns" and to form projects to act towards the modus vivendi (way of living) they want to achieve (Archer, 2012, p. 1). To access the internalised processing of the structure-agency, Archer developed a qualitative methodological approach based on three modes of reflexivity communicative reflexivity, autonomous reflexivity, and meta-reflexivity. A fourth fractured reflexivity - was discovered in Archer's application of her model, as well as ten mental activities of internal conversation used by her working class participants (Archer, 2003). Mode descriptions were refined following further testing on defined social class groups (working class, university students, and university staff within the United Kingdom) (Archer, 2007, 2012). Archer believed people were either passive agents or active agents.

Archer's reflexivity theory and internal conversation methodology have attracted considerable debate for the perceived lack of attention to socio-cultural and social construction theories of socialisation and relationships in the transfer of knowledge (Akram \& Hogan, 2015; Farrugia \& Woodman, 2015; Mutch, 2004). The rigidity of the critical realist approach is seen to decontextualize and reduce the importance of social interactions outside of structural-agency processes (Burkitt, 2016; King, 2010) and the place of emotions in reflexive thought (Burkitt, 2012; Holmes, 2010). Despite these criticisms, her methods offer great insights and Archer's modes of reflexivity and internal conversation methods have been applied in biographical studies in Portugal (Caetano, 2017b), South Africa (Case, 2013; Luckett \& Luckett, 2009) and Poland (Mrozowicki, 2011) and a small number of life stories and decision-making studies in higher education 
(for instance, Baker, 2019; Barratt, Appleton, \& Pearson, 2019; Dyke, Johnston, \& Fuller, 2012). These studies looking at internal conversation are based on naturally occurring events or life decisions within countries with historically long traditions and dominant monocultures. What has not yet been considered is how Archer's methodology and modes of reflexivity apply in countries with younger and more dynamic histories, nor where an intervention challenging an individual's values and concerns has occurred. My thesis offers a study which applies Archer's method and modes with a diverse group of higher education students and with a deliberate intervention in the form of a core set of citizenship courses.

\subsection{Outline of the thesis}

The layout of this thesis is as follows. In chapter 2, I present the theoretical and conceptual frameworks which I use throughout this thesis. This framework is informed by sociological theory of reflexivity and Archer's critical realist concepts of the relationship of structure-agency in reflexivity and the internal conversation (Archer, 2003, 2007, 2012). I also explain how I incorporate Berger and Luckmann's (1966) "social construction of reality" theory of how reality appears to individuals and groups and reflect on Archer's disagreements with Bourdieu's habitus and social constructionist approaches, to understand the importance of social contexts on reflexive practices.

I explain the methodology and modifications I applied in this research in Chapter 3. I describe the need for a qualitative longitudinal research methodology and how I used strategies reflecting Mātauranga Māori (Māori ways of knowing and being) to overcome recruitment and retention issues. The sequence and nature of the repeat narrative-style, semi-structured interviews used and the reasoning behind the collection of written artefacts are presented. I reveal how I used Archer's mental activities in interviews and modes of reflexivity in analysis. In this chapter, I also justify the novel use of framework analysis as a lone researcher and detail ethical considerations of the research.

In Chapters 4 and 5, I report on the findings from my research. My focus in Chapter 4 is on the how the participants' contexts and commitments affected their attitudes towards their study in three core citizenship courses. I follow this with description of the participants' experiences of study at each level (100-/first-year level, 200-/second-year, and 300/third year-level), and how their changing circumstances affected their ensuing involvement in the project. In Chapter 5, I present vignettes or case studies for nine participants, seven who completed all three citizenship courses in the study time frame and two whose life-projects challenged their thinking of citizenship. My findings, from 
applying Archer's modes of reflexivity in my study, indicate that Archer's claim of a reflexive progression in dominant modes (from communicative reflexivity to autonomous reflexivity to meta-reflexivity) as a consequence of increased education is too simplistic. In these two chapters, I incorporate sections of students' narratives to allow their words to demonstrate their reflective considerations.

In Chapter 6, I elaborate on the discoveries that emerged from my research project findings. In particular, I consider how past life experience influences the presence of communal or individual values and how social interactions are used in conjunction with internal conversation. I propose that there is no 'normal' reflexive mode associated with education level for these diverse university learners, but personal events and social contexts are stronger determinants in reflexive processing of value-laden citizenship knowledge.

In the second half of Chapter 6, I present a tentative layered framework of the important characteristics of higher education students' reflexive deliberations in considering citizenship values. In Chapter 7, I review and draw together the findings from this research to conclude the thesis and to offer suggestions for future research in this area.

\subsection{Chapter summary}

Post-compulsory education is occurring in a changing world, and researchers have not yet paid enough attention to how higher education students' process the knowledge and embedded values taught in university courses in light of their own lives and personal beliefs. Archer's theory of the structure-agency relationship offers a useful starting point for considering this gap in understanding. In the next chapter, I develop my argument for using Archer's theory of reflexivity and internal conversations to understand higher education students' ability to act on new knowledge in considering their social reality. 


\section{Chapter 2. Theoretical Framework}

\subsection{Introduction}

In this chapter I locate the rise of reflexive thinking within critical realist sociological theory, my considerations of reflexivity theory and social construction theory, and its applications in higher education research. I start by establishing reflexivity's place in sociology and in a postmodern world. I explore Margaret Archer's theorisation of reflexivity and individuals' internal conversations and deliberations. In doing so, I make the connections between Archer's critical realism and social construction in understanding how individual realities are formed and change through socialisation. My intent here is to position how reflexivity has been and can be used to explore individuals' realities and possibilities for change. Looking at an individual's reflexivity can expand our understanding of the complexities of the intersection of students' personal realities and learning in higher education. This is pertinent in a globalised and rapidly changing world where new ideas, values and priorities are introduced into peoples' lives from multiple sources daily. Within higher education, policy makers and teachers need to be attuned to the shifts in societal realities.

\subsection{A dynamic world}

Sociological theories recognise the social world (humanity and its environments) as interconnected and continually undergoing change. Such changes affect concepts of, interactions with, and the nature of the relationship between people and the world $(A$. Elliott, 2014; Giddens \& Sutton, 2014). In western and non-western societies, the social institution of higher education has been and is considered by many as a place for promoting and enabling the transmission and debate of knowledge and the development of skills for employment (Ashwin, 2014; Jarvis, 2007). Internationally, a higher, university or post-compulsory, education qualification has become accepted as a necessity for selfprogression and societal advancement (Ministry of Education, 2014; UNESCO World Conference on Education, 1998). Similarly, within an increasingly globalised world, sociological concepts of work, family, citizenship, gender and their meanings are being reassessed. Globalisation, virtual learning and the internationalisation of education are being socially defined and accepted as norms within education (Klemenčič, 2018). Sociological theories seek to understand how society works and understandings of social 
phenomenon are developed, understood (Giddens \& Sutton, 2014) and incorporated in people's lives. Social theory provides a means for researchers to examine how wider social changes affect individuals' understandings of themselves, their social world, their values and concerns, and to explain how able individuals feel they are to adapt to a changing world.

In modernity, traditional structures and national boundaries of peoples, cultures and their associated knowledge were clearly defined and easily replicated to maintain the status quo (Giddens, 1991). People knew their place and roles in society. However, since the mid-twentieth century, technological advancements, new ways of working, and social and geographic mobility have increased rapidly, leading to a period of extended reflexive thinking in people (Archer, 2003, 2012) in an era of 'reflexive modernization' (Beck, Bonss, \& Lau, 2003; Beck et al., 1994). Rather than change in society being absorbed by the collective, the risks associated with change become an individual concern (Beck, 1994). Instead of knowledge, values and norms being passed from one generation to another receptive generation, new cultures and ideologies emerge and are examined, and new ways of thinking and being are integrated into society. People are exposed to such new ideas which they evaluate and weigh up against their existing values and life concerns. As a result, there has been a shift in the nature, dynamics and interpretation of knowledge, particularly within traditional western societies (Gergen, 2015), and changes in the reference points used in people's decision making.

The breakdown of traditional boundaries, and challenges to the western meta-narrative of a common and shared collective knowledge, has seen researchers seek to understand the new connections between open and inclusive reasoning, personal knowledge and societal action (Archer, 2000, 2003; Crotty, 1998; Tsekeris \& Lydaki, 2011). Within social theory, increased consideration of reflexive thinking seeks to understand or make sense of the macro- and micro-interactions of social life and individual agency (Archer, 2007, 2010a; Clegg, 2005, 2015; Giddens, 1994). The main concerns of sociology are understanding social dualisms (Archer, 2000): the individual and their relationships with society (Archer, 2007); human action and social structures and which has priority; social and systems integration (where individuals or actors fit within different parts of society) and the effects of capitalism and socialism (Crotty, 1998), and how self-knowledge is developed and expressed (King, 2010; Susen, 2016). In the 1980s, British sociologist Margaret Archer joined discussions on the nature of the self and reality (including Berger \& Luckmann, 1966; Bourdieu, 1998; Bourdieu \& Wacquant, 1992), the relationships and interactions between social knowledge, structures, and agency (Archer, 2000) and the 
reflexive nature of people as agents (Archer, 2003, 2010b; Beck, 1994; Giddens, 1984, 1994; Lash, 1994)

Archer's key criticism of the turn in social theory was that the elevation of individual action continued to skew the relationship between structures and agents. In seeking to bring back balance, Archer's arguments focused on a range of theoretical positions including the works of symbolic interactionists (Mead), Bourdieu's structuralist account of human behaviour, and social constructionists' view of reality (including Gergen and Berger and Luckmann). Her reasoning targeted the merging of action and social structures, and selfhood (identity) and self. Fundamental to Bourdieu's cultural structuralism and Berger and Luckmann's social constructionism arguments were that social history, culture, language and experience frame peoples' on-going knowledge of reality (Berger \& Luckmann, 1966; Bourdieu, 1998). These epistemological positions, influenced by the earlier works of symbolic interactionist Mead, structuralist Durkheim and rationalist Marx and others, considered there to be a gradualism or intertwining in the structure - agency relationship. Neither Bourdieu nor Berger and Luckmann made ontological statements on reality; external reality was accepted as existing in physical form and in the knowledge and values held in social structures associated with groups of people. It is the social interaction with reality that forms our understanding of what is real. However, Archer's principal objections to these theories were ontological: Archer believed that reality exists externally to people and their perception of reality, and the social world was the result of interactions in the dualisms of structure and agency (Archer, 2000, 2003). By holding the separation of reality from personal knowledge of reality, Archer could elaborate on the duality of structure and agency. Thus, the key debate is ontological dualism against epistemological gradualism or constructionism.

The dualism of structure and agency is critical in Archer's reflexive approach to the interaction between the two. Archer argued that Gidden's structuration, Berger and Luckmann's social construction of reality and Bourdieu's cultural capital and habitus theories do not make sufficient separation between the powers and properties of people, and the powers and properties of structures. "Ontologically, 'structure' and 'agency' are seen as distinct strata of reality, as the bearers of quite different properties and powers. Their irreducibility to one another entails examining the interplay between them" (Archer, 2003, p. 2). However, I believe Archer's arguments against social construction's framing of socialisation as reinforcing structure and agency at an ontological level are misguided, her focus is the ontological objectivity of dualism. Bourdieu and Berger and Luckmann's discussions of reality, its agents and arrangements are epistemological, objectivity is the 
result of 'habitus' and 'cultural capital' (Bourdieu, 1984) 'habitualization' and 'institutionalization' (Berger \& Luckmann, 1966; Berger \& Pullberg, 1965). They are concerned with how individuals know what reality is and interpret it, rather than what reality is (Crotty, 1998). In taking this position, Archer limits the possibilities for including different levels (that is, individual or group) of personal understandings of reality in her own analysis of the interactions between structures and agency.

Archer set out to address the failure of theorisation on the interplay between structures as external reality, how agency worked, and the influence of these interplays on agents' decision-making and actions. Archer argued these earlier theorists had taken short cuts in "downwards conflation" - determining that structures dominated the acts of people or in "upwards conflation" - attributing agents with too much power in shaping the parts of reality (Archer, 2000). Holding structure-agency as distinct entities with similar power is critical to Archer's explanations of how individuals respond to social change.

To incorporate this distinction, Archer proposed a morphogenetic approach as an explanatory framework for examining the effects of structure and agency on each other and the social changes that result (Archer, 2010a). In the morphogenetic approach, ideas or beliefs are held as habits, reflexively affirmed within society, consciously and subconsciously, through individuals' shared understandings of how the properties and powers of structures produce particular social outcomes. Morphostasis occurs when the existing social order preserves or replicates structures through social conditioning. However, morphogenesis occurs when parts of society push back against structural entities and their powers and thus brings about change or development in structures over time (Archer, 2003, 2010a). Archer's position is that morphogenesis is a necessary social process that enables individuals and social groups to cope in an increasingly globalised world.

I agree with those social researchers who argue Archer's morphostasis is similar to Bourdieu's concept of habitus (Akram \& Hogan, 2015; Elder-Vass, 2007; Farrugia \& Woodman, 2015). Habitus captures the stability of embodied understandings and practices and how they result in a person socially engaging without having to think about what they are doing (Bourdieu \& Wacquant, 1992). This can be either an intentional or an unconscious means of coping with the complexities of social interactions. Archer argued in a postmodern world or changing society, the reflexive agent is actively advocating for change in social thinking or for the continuation of structures. For Archer, habitus and Bourdieu's agent is passive in conforming or reproducing what exists in society. In contrast, Archer's agent is continuously and intentionally moving towards 
change and in the process they and their social groups are undergoing transformation or double morphogenesis (Archer, 2003). Morphogenesis can account for micro-, mesoor macro-change in the individual or society (Archer, 2003, 2010b).

Archer's argument on cultural morphostasis, the result of the stability of ideas within a population - where existing structures have power and status (Archer, 2010a) - is similar to social constructionists' Berger and Pullberg's concept of reification (1965). Both refer to the objectification of structures, where structures are named by people, characteristics are ascribed, and understandings remain constant over time. For example, higher education is understood to be a process of instruction or the passing on of knowledge, within a university or some other formal system. To Archer, entities are viewed as existing externally to society through morphostasis and continue to perpetuate. Similarly, in social construction, power is given to an entity through alienation (or distancing from the self) and in reification social forms become a standard object of reality; however, reified entities are also nested and referenced in the individual's consciousness or understandings of their current social context (Berger \& Pullberg, 1965). This link to active, conscious (and therefore current) individualised reinforcement of accepted social patterns and structures provides an alternative analytical point to Bourdieu's habitus in considering how individuals make their way through and contribute to wider social change. It also captures Archer's notion of "social conditioning; this is where the prevailing structural and cultural contexts shape the situations or social worlds that agents find themselves in (Archer, 2000, p. 55).

Archer's epistemological framework offers possibilities for understanding the interplay of structure and agency within learning contexts that may not be evident using other approaches. Ontologically Archer's critical realism and Berger and Luckmann's social constructionism appear to be at odds. Although not evident in a first reading of the theories, it could be argued, however, that there are areas where they complement. At an individual level, a reflexive (Archer, 2003) or conscious (Berger \& Luckmann, 1966) 'sense of self' is produced in socialisation and is needed to challenge ideas, and to bring about morphogenesis. They both present arguments that much of human social life appears to be a part of normal and natural reality. Individuals and society don't construct reality but construct beliefs about reality, which are then sustained through social interaction. In everyday life, people unconsciously and consciously interpret and evaluate "what is real, how reality works, which factors cause what outcomes, and what truth claims can be trusted" (C. Smith, 2010, p. 167). Physical and social structures are a part of social and personal reality. Collectively, the personal power generated through 
reflexivity may be used to bring about individual or group courses of social action. While Berger and Luckmann do not offer a way to examine the entwined structure and agents, Archer's separation of structure-agency and internal conversation methodology provides the means to examine the interactions between the two which generate or limit agency.

\subsection{Critical realism, structures, agents, agency and reflexivity}

In the previous section I introduced Archer's keys concerns on the theoretical positioning of the structure and agency relationship within sociology. In this section, I present the arguments from critical realism which underpin the relationship between structure, agents and agency revealed in reflexivity. I then introduce Archer's modes of reflexivity - her methodological approach to understanding the structure-agency relationship.

\subsubsection{Archer and critical realism}

Archer's work on reflexivity extending the modernisation argument was influenced by the philosophical and sociological works of American pragmatists Peirce, Dewey and James, British sociologist and philosopher Giddens, and critical realist Bhaskar (Archer, 2003). From the view of pragmatism, no absolutes exist; the meaning and truth of an idea is revealed in its outcome. This position countered the dominant view of absolute truths of scientific positivism. Archer followed Giddens' position that society's structures were products of and reproduced by human action and human agency (Giddens, 1984, 1991) or as King described "a virtual order of differences and rules" (King, 2010, p. 253). Giddens's structuration theory depicted "a duality of structure" where individuals reinforced institutional structures through their actions (Giddens, 1984, p. 19). The theory was a means of accounting for the global social forces at work in social change at an individual level - as traditions of culture, gender, family, community, work and other aspects of the social world were open to redefinition (Archer, 2000). Giddens's view of collective social action as dependent on an individual's agency, where the rules and norms of social structures would enable or constrain agency, fit within Archer's developments on personal and collective agency (Archer, 2000). Social change would affect one's daily actions and self-identity. Consequently, individuals would need to view their self-identity to be "a reflexive project of the self" open to construction:

Self-identity, then, is not a set of traits or observable characteristics. It is a person's own reflexive understanding of their biography. Self-identity has continuity - that is, it cannot easily be completely changed at will - but that 
continuity is only a product of the person's reflexive beliefs about their own biography (Giddens, 1991, p. 53)

Likewise, Beck et al.'s (1994) discussion of 'reflexive modernization' corresponded with the view that the social structures encountered in individual life experiences were ordered by knowledge. Beck considered self-knowledge to be set within wider state and political systems which influenced people's daily experiences of them. The risks of system change in globalisation would alter social structures and individuals would have to calculate how they responded to that (Beck, 1994). Giddens's interest was on challenges to such structures in the development of a global society and how the individual would respond to these changes (Giddens, 1994). Beck (1994) and Giddens (1994), however, agreed people needed agency to deal with on-going biographical change forced on them by structural and social change. Although Beck saw structures as having greater power over individuals, Giddens saw the individual as being able to be responsive to structural enablements and constraints - pushing back on them in reflexive monitoring of their actions. Giddens focused on reflexive consciousness and action, arguing some human actions are unintended and can have unforeseen consequences which shape future actions (Giddens, 1994); thus, wider societal interactions can influence the individual's life path.

Critical realists (including Bhaskar, Sayer) recognised the known world was changing and new ways of thinking about the world were required. Replication of existing ways of thinking about the world were insufficient and reductionist (Bhaskar, 2014). Meanwhile, Sayer (2010) believed insufficient consideration of people's internal conversations was made in the social sciences, despite society's position that a person is responsible for their actions. The critical realist's approach then was to understand the causes of experiences or events by focusing on interpretations in description and hermeneutics, rather than explanation and causality. Archer and colleagues (2016) acknowledged that there was no one set of defining beliefs, methodology, principles or characteristics, rather critical realists have developed different strands of thinking coiled together. This is reflected in Archer's theory development where she drew on, critiqued and challenged a range of sociological theories. However, Giddens and Sutton (2014) also described critical realism as "a research method that, its advocates argue, is capable of getting below the surface of observable events to gain access to the underlying causes or 'generative mechanisms' of real-world phenomena" (pp. 33-34). Thus, critical realism is considered within sociology as both a means of looking at generative mechanisms of reflexivity and development on the work of classical theorists to bring in contemporary 
understandings of reality (Giddens \& Sutton, 2014). Key to critical realism are ontological realism and epistemic realism.

\section{Archer and social reality}

Within ontological realism, there is a view that much of reality exists and operates independently of our awareness or understanding. Many features of the world cannot be empirically observed or quantified or fully explained. Instead, critical realists seek to characterise the nature of social reality by combining explanation (theory) and interpretation in observation of cultures, social structures, people and human interactions and actions (Archer, Bhaskar, Collier, Lawson, \& Norrie, 1998; Archer et al., 2016; Bhaskar, 2014). In doing so, the nature of the internal connections between cause and effect, structures and processes, and complexity and diversity within the social world, is revealed independently through the perceptions and interpretations of individuals (Ritchie, Lewis, McNaughton Nicholls, \& Ormston, 2014). In critical realism, society is an open but stratified system of structures which people, as agents, inhabit (Archer, 2003) and have their own properties and powers "which include a reflexivity towards and creativity about any social context which they confront" (Archer et al., 1998, p. 190). To exercise this power within the enablements and constraints of society is to have agency.

Archer was clear on separating realist ontology from epistemology and interpretations; it is this stance which plays a part in her opposition to social constructionism as a means of understanding reality. Archer's ontology is that reality exists externally to the person and there are no absolute truths. Critical realism's epistemic realism where "[o]ur knowledge is context-, concept-, and activity-dependent" (Archer et al., 2016, p. 6), was similar to Berger and Luckmann's epistemological position except they elevated the role of the social in the development of knowledge. Both Archer and Berger and Luckmann believed knowledge and a person's understanding is "always historical, perspectival, and fallible, entailing ... the necessity of methodological pluralism" (Archer et al., 2016, p. 6). What is missing from Archer's thesis is how social relationships influence understandings of structures.

The temporal and spatial nature of the social is a strength and weakness of critical realism. If reality takes the form of structures that exist externally to the individual or agents and form internalised reference points or beliefs (Archer, 2000; Archer et al., 1998), then the context of present time and social conditions needs to be considered as well as historical influences. An individual's perspective of what is real is not framed alone but formed with a myriad of others over the current and distant passages of time. 


\section{Archer and individualism}

Ontological realism and the approaches used by Giddens (1994) and Archer (2000, 2003) have been criticised for their emphasis on individualism (Burkitt, 2016; Caetano, 2015a). For instance in earlier responses to this claim, Archer further developed her notions of primary and corporate agency (2000) describing them as relational or collective agency, which requires individuals to work together (Archer, 2012). However, to participate in collective agency she proposed the individual must convince and take others with them to address their own concerns (Archer, 2012). Archer argued that postmodernity's changes affected each person in their pursuit of their ultimate concerns. However, as Gergen (2011) debated, the self and the concerns one holds may be developed in collective reflexive deliberation and within social traditions and conveniences, as well as in isolation. Beck and Gidden's contemporary, Lash (1994) was concerned with structuration detaching the person from their social contexts, interactions and influences of external reference points. The argument on separation of the individual from their context continues to be levelled at Archer's theory and methodologies (Caetano, 2014; Chalari, 2013).

Archer's morphogenetic theory was a response to Giddens's focus on structuralisation. Her goal was to add more emphasis on the role of the individual in order to support a fuller picture of the agent. As criticisms of her theory were made, Archer (2008, 2012, 2010b) responded reflexively, pushing back in the debates, resisting or modifying her work to demonstrate her position as an active participant in the sociological debate. Archer argued that Giddens conflates structure and agency elevating the social over structures, but her morphogenetic approach restored balance as it referenced a person's temporal contexts (Archer, 2000, 2003); however, her critics (including Caetano, 2015a; King, 2010) disagreed. While explanations of individuals' reflexive practice within contexts at one point-in-time provides a snapshot of the structure-agency interaction, social contexts and exchanges change over time independent of the individual (King, 2010). Portuguese sociologist Caetano (2015a) argued that time effects on reflexivity can tell us more about how understandings and priorities are deliberated on, but these need to be considered within the different features of their social contexts. Hence, it is important the contextual conditions around a person's reflexive deliberations are considered when looking at structure-agency effects.

In more recent writings, Archer has joined with Italian sociologist Donati to elaborate on the critical realist position of 'collective reflexivity' (Archer, 2013) and 'relational reflexivity' (Donati \& Archer, 2015) to define the 'relational subject' (Donati, 2016). Similar to Berger 
and Luckmann's (1966) socialisation, in relational realism the subject is "'relationally constituted' ... in as much as they acquire qualities and powers through their internal and external social relations" (Donati, 2016, p. 355). Thus, relational reflexivity extends the idea of a single person's reflexivity to their social group. Reflexivity is consciously used by the group to work out how their network is influenced by the relations with 'others', or those outside the group (Donati, 2016). It is the quality of the relationship which influences reflexive thought on the relationship, thus the relationship itself is a 'relational good' that is acknowledged (Archer, 2013). This proposition of a differentiation between individual or subjective reflexivity and collective reflexivity extends Archer's theory of reflexivity; however, there is an ongoing tension about how the relationship between relational reflexivity and agency at a collective level works.

\section{Archer and Bourdieu}

Other critics focused on Archer's rejection of Bourdieu's habitus, (Akram \& Hogan, 2015; Elder-Vass, 2007; Farrugia \& Woodman, 2015) and the elevation of inner conversation over other social exchanges (Sharrock \& Tsilipakos, 2013). Elder-Vass (2007) argued that not all responses are part of conscious thought, but are engrained in cultural, practical or social responses, routines and habitualised behaviours through social conditioning. Akram and Hogan (2015) emphasised the importance of using heuristics to consider the patterns of language and mental short-cuts used in reflexive thought; to look at how people saw themselves in relations to others. Greek scholars Tsekeris and Lydaki (2011) argued sociological discussions based on reflexivity, self-identity and selfawareness were a part of western or Eurocentric traditions of phenomenological individualism and agency. Tsekeris and Lydaki (2011) proposed Mouzelis's apophatic reflexivity as a more flexible and responsive theory of change which incorporated spiritual elements, another feature ignored in Archer's reflexivity. This argument moves the discussion towards greater consideration of the subconscious influence of culture and emotion in individuals' reflexive processing. In keeping with their arguments, Archer's development of an individualist concept of reflexivity based on social isolation from the predicted effects of technological and social global change seems over-reactive. Archer's stripped-down version of the nature and place of reflexivity in individuals requires augmentation. What is evident is that the debate over Archer's theory of the structure-agency interaction continues and is being extended to include elements that are important in other cultural systems such as faith and allegiance.

Despite these criticisms and Archer's judgments on the works of other sociologists, the use of the dualisms of structure and agency does allow for an unravelling of institutional 
and social webs of influences in personal reflexivity deliberations. In the next section, Archer's perspective of structures, agents and agency are presented in preparation for more in-depth discussion on reflexivity's modes and the reference points of reflexive thinking - values and concerns, and the dialogues of internal conversations.

\subsubsection{Structures}

As discussed earlier, the reality of structures and the experience of agency are considered two important entities in critical realism; Archer's focus was on the interactions between the two. If structures are objective and agency involves some degree of subjectivity they are, therefore, ontologically separate (Archer, 2003). Structures represent society's influences on individuals and agency - the freedom and the constraints that individuals have to act and shape (Giddens \& Cassell, 1993) or bring change to society. American pragmatist Parsons proposed social structures have normative expectations of the individual and their behaviours (Giddens \& Cassell, 1993). Social structures like the economy, identity, family and social class are the result of social interactions over time and influence individual actions. As discussed previously, Archer (2003) positioned her morphogenetic model of social transformation against the conflation of structure-agency in Giddens' (1984) structuration theory. In doing so, she was trying to separate the properties of structures from their powers or enablements and constraints. Archer saw structures as pre-existing individuals, but agents could act independently of structures whereas for Giddens, structures and agency coexisted and agents shaped structures over time. Nevertheless, knowledge of the nature of structures is necessary to understand agency and how individuals, or agents, are affected by, reproduce or transform structures over time.

Structures have powers that are nested in their characteristics or properties (Archer, 2003). The way in which entities are organised, distributed and have roles form their structural emergent properties. The ideals, beliefs and principles which define their existence are cultural emergent properties. The enablements and constraints of these properties are encountered by agents, or people, when they set out to undertake a particular action or to work on a project towards a specific goal. For the power of enablements and constraints to be exercised, there needs to be compatibility or incompatibility between the structures and the proposed project, and agents have to respond (Archer, 2012). In responding, the agent exercises their personal powers. While Archer positioned structures and their properties as existing externally, others believed society plays a part in creating, naming, and recognising structures' powers through cultural conditioning and reproduction (Bourdieu, 1984), in their social processes 
(Gergen, 2015), or in intersubjective shared understandings (Berger \& Luckmann, 1966). How external and internal references to structure can be integrated into considerations of structure-agency remains under debate.

Unlike the social and cultural construction positions where structures and agents are intertwined in social interactions (Berger \& Luckmann, 1966; Bourdieu, 1984; Gergen, 2015), Archer's critical realism placed structures and agency as dualistic and the social as the interaction between these distinct entities. Archer's works $(2000,2003,2007)$ outlined the interplay between structures, as objectified reality, and independent agents. The interaction influences agents' ability to act. If too much emphasis is placed on the power of structures, or the power of the individual, the role of reflexivity in agency is conflated. Conflation privileges the power or influence to one aspect or entity over the other (Archer, 2000, 2003). Thus, social construction was seen to elevate the powers of the social too much over structures, and structuralism elevated structures over people.

People assume structures (such as gender and class) exist through social conditioning (Archer, 2012) or socialisation (Berger \& Luckmann, 1966) where social resources and beliefs are passed on. Where socialisation was concerned with relationships and social understandings, Archer's social conditioning looked at the interplay between structural power and cultural power, and how these impinge on agents who have personal power to act one way or another (Archer, 2003). Archer attributed both structure and agency with equal powers of cause and effect. She argued that if social and personal concerns align and values were integrated this would represent contextual continuity. Contextual discontinuity occurs when individuals no longer hold the same values as their natal or traditional context, and contextual incongruity occurs when an individual's values are in conflict with those around them (Archer, 2003, 2007, 2012). The interplay between social conditioning and agential power reveals social enablements and constraints of structures which act on the individual's activities (Archer, 2007). Through reflexivity, the individual examines what is happening around them and makes decisions about actions or activities to continue, to change, or to cease.

Some critics (Akram \& Hogan, 2015; Elder-Vass, 2007; Farrugia \& Woodman, 2015) deliberated on Archer's social conditioning as another version of Bourdieu's structural habitus at an individual level, while Archer (2003) argued habitus is deterministic in reproducing the existing social orders and therefore unable to respond to rapid change. Others believed habitus describes where people fit in terms of their social origins (ElderVass, 2007), social and personal identity or positions (Caetano, 2014; Farrugia \& Woodman, 2015) and their social roles (Caetano, 2014); knowledge of habitus is 
reasoned to be critical to understanding current thinking and responses. Akram and Hogan (2015) suggested Archer had gone too far in attempting to address the balance between the agent and structure, resulting in the privileging of reflexivity above individuals' agency or responsiveness to change. Archer disputed this as she saw structures and agency as entwined where they "emerge, intertwine and redefine one another" over time in "structural elaboration (morphogenesis)" rather than the "structural reproduction or (morphostasis)" of habitus (Archer, 2012, p. 52).

Farrugia and Woodman (2015) addressed Archer's point that habitus does not provide an account for how people live out their ultimate concerns in periods of disruption, which is at the heart of Archer's structure and agency debate. Instead, Farrugia and Woodman (2015) present Bourdieu's habitus as an encompassing condition of humanity, and reflexivity takes place within habitus. If habitus accounts for structure, it is through habitus people work out their ultimate concerns. Archer does not identify the origin or foundations of ultimate concerns. If ultimate concerns are not derived from socialisation or habitus as Archer argues, then Farrugia and Woodman (2015) claim internal conversations on structures must be referenced on previous internal conversations. This produced a shallower concept of reflexivity than using Bourdieu's habitus. Along with Elder-Vass (2007), these critics' concerns were that not all human responses to structures are conscious and intentional. Social existence and history leaves traces in individuals' memory which become reference points for on-going reflexive thought. This may well be the case in Archer's autonomous reflexivity (Chapter 2.6) where internal conversations act out in response to disruptions or negligence in their childhood upbringing. Incorporating the historical influence of structures on individuals' current responses would bring further depth to understanding the intersections of structureagency.

\subsubsection{Agents}

The structure and agency interaction is dependent on the presence of agents. Archer (2000) defined agents as human, relational beings with "forms as Selves, Persons, Agents and Actors, and thus set limits to their variability" (p. 17). Central to critical realism is the argument of how much an agent is a neutral being or societal artefact. A Marxist reductionist approach would present action as hard wired in agents' brains devoid of choice. The opposing holism position of Durkheim would place the subjective self as a full construct of society, the result of societal interactions (Giddens \& Sutton, 2014). Archer followed Giddens (1984) in locating her theory in the middle ground between these positions, presenting the agent as a subjective individual with choices but 
conditioned by objective properties of society (Archer, 2000). While social context is important, Archer limits understanding of the social to free agents or individuals' subjectivity in determining their plans before acting, thus ignoring the role of habitus. They have subjectivity, a "personal interior property, with a first-person subjective ontology, and with the powers that can be causally efficacious in relation to [themselves] and to [their] society" (Archer, 2003, p. 14). The individual recognises there is a disjuncture between the properties and powers of the social world and their own; these social features enable or constrain their choices of action. Archer did not focus on selfknowledge or societal knowledge, but on how structures impinge on agents and how an agent's power affects their reception in society.

Consequently, the biographical, relational and emotional reference points of self, and the immediate interactions of individuals acting within their wider society, are according to Archer, diminished by social forces. So while the reflexive deliberation of social agents is the strength of Archer's approach in examining structure agential tensions, it is unclear when a person becomes an agent or whether they always exist as agents (Jarvis, 2007). Nor how views of themselves and enablements and constraints may change over an individual's life-course (Cieslik, 2006). In ignoring the complexities and the uniqueness of an agent's situation and their understanding of the contours which shape their concerns and agency, Archer presents a partial picture of the structure-agency relationships. In a similar fashion, using a biographical analysis of reflexivity over a lifetime (for example Caetano, 2015b) can miss the nuances of specific or focused events on reflexivity and agency.

Agents may be strategically responsive, but not always knowledgeable in their actions or have intentional outcomes in mind (Burkitt, 2018; Caetano, 2019). In considering the usefulness of Archer's dualism of structure-agency in the higher education context, South African educationalist Williams (2012) maintained that it was not clear if university students believed they are agents and are able to act back on the structures around them to bring about changes to structures and their associated cultures. Teaching practices using reflective exercises in the "performative environment" of higher education were seen by Macfarlane (2017) as limiting or supressing the powers of agents, as specific social and employment values and attitudes were being taught and assessed. For example, in response to the growing use of reflective writing in assessment, students strategically picked the stories they presented to meet assessment criteria (Macfarlane, 2017). Consequently, reflective writings alone are not necessarily indicators of personal development, or agency. 
For social identity theorist Burkitt (2018), the biggest flaw in Archer's argument was that it places agents as existing and acting alone in their reflexivity. Agents deal with the enablements and constraints of external structures by themselves. Instead, by taking a relational view of the powers of structures as products of socialisation and social interrelations, an individual's agency can be considered as interdependent and influenced by others (Burkitt, 2016). Using the duality of the structure-agency relationship and Archer's concept of reflexivity through internal conversation as the medium for analysis of the effects of structures on agents and agents on structures, it should also be possible to identify the social positions and relationships which influence reflexive processes. However, it is also important that multiple methods are used to collect data to reduce reliance on evidence that may have been generated to meet performance criteria.

\subsubsection{Agency}

As agents encounter structures their agency is revealed. Relational sociologists Emirbayer and Mische (1998) see the focus on agency as a move from reflective thinking on the past to focusing on future possibilities in the interplay of structures and agency. They consider both the focus on human agency (as used by Bourdieu and Giddens) and alternatives (such as rational choice and phenomenology) as one-sided. Archer (2000) summarised her critical realist position on agency as the condition of being able to act for oneself. Rational thought is seen in active agents having concerns or values which they care about and use in their considerations of the possibilities of actions. It is how these concerns or values were formed that Archer and Bourdieu disagree on. As discussed earlier, for Bourdieu, the relationship between the subjective and objective is embedded in the power of the individual in everyday life.

Archer proposed that reflexive people are all agents and given the right social conditions have agency. Influenced by Marx, both Archer and Bourdieu saw agents as occupying spaces where embodied, objectified and institutional capital influence people's place in society (Archer, 2000; Bourdieu, 1984). Life is ordered within the natural, practical and social worlds which are separate and intersect with personal subjectivity as the person moves through the world. With the social world broken into distinct fields (Bourdieu) or structures (Archer) neither these nor agents are independent entities. Bourdieu considered social agents as products and producers of acts within particular contexts or fields and "knowledge of the social world has to take into account a practical knowledge of this world that pre-exists it" (Bourdieu, 1984, p. 467). Archer considered everyone as an agent - an involuntary collective position relative to the resources available in society. 
The agent, by choice, aims to take a role in society where they can act upon their values. (Archer, 2007). However, for Bourdieu cultural capital formed in traditional social structures of habitus - family and community - is the foundation for determining agency not individual action. In the social world where traditions are broken, the individual struggles.

Archer's main argument was that habitus was reliant on a "pre-constructed" world (Bourdieu \& Wacquant, 1992, p. 235) - if society is undergoing change, how can habitus as the embodied social structures and history of people present in their dispositions (Bourdieu, 1984) keep up? If the way in which the individual perceives the world, and acts on it, was contingent on having a traditional store of knowledge of pre-existing structures - then these reference points diminish the sort of reflexivity Archer believed was required to actively respond to change (Archer, 2007). She also argued that routine behaviours do not provide answers for every contingency and action (Archer, 2010a). Although Archer raises these important issues, dismissing the influence of habitus from primary and secondary socialisation altogether reduces the opportunity to explore these underlying characteristics on individual agency. During early and later adulthood, family history and wider social experiences remain reference points for on-going reflexivity. Some behaviours (e.g., young students returning home between university study periods) are likely predispositions towards family expectations and emotional ties rather than rationalised behaviour. Indeed, in Archer's (2007) analysis of the internal conversations of her participants, she gives occupations as an indicator of their presence within a particular British social class. Archer expects, presumably through habitus rather than social conditioning in a rapidly changing situation, her reader to understand the link.

The contextual and conditional nature of agency is elaborated on by other writers. Emirbayer and Mische (1998) view agency as entangled within a situational context. It is this context which shapes the way in which people are able to control their responses. Contexts affect how agents interpret their situations or construct their responses, the choices they perceive they have and the possibilities for action. Agential capability in shaping responses may be different, enabled or constrained in situations which are temporarily constructed with others (Biesta \& Tedder, 2007). Opportunities for action are not devoid of emotion as often emotions are an integral part of some social interaction (Burkitt, 2016). Conflicts between individuals in their understandings of situations and different priorities and possibilities may leave an agent in a void unable to act. This makes it important to consider the agent's perspective of how they are able to respond. Some form of biographical method of collecting perspectives is important to understanding how 
people evaluate and reconstruct the possibilities for their agency (Biesta \& Tedder, 2007; Caetano, 2015b). Agency has historical, temporal and social references, and these are precursors to actions which change social reality. Their presence in internal conversation is central to Archer's reflexivity and its associated modes.

\subsubsection{Reflexivity}

Reflexivity is a characteristic of human agents. The term reflexivity has been used at a personal level in the conscious and sub-conscious processes at work in the mind, in which a person considers themselves in relation to their society (Archer, 2003; Caetano, 2014; Giddens, 1991, 1994). For Archer (2003), to be reflexive was to engage cognitively in the social world. Reflexivity "is the regular exercise of the mental ability [by people] to consider themselves in relation to their social contexts and vice versa" (Archer, 2007, p. 4). Archer (2000) and Giddens (1984) reasoned that in traditional societies people as passive subjects were controlled by society's structures. With globalisation, traditional social structures are believed to have broken down under influences of market forces and political and social interaction. Critical realists' concerns were that the entangled and infused nature of the external and social worlds in late modernity would stop people from acting for their individual and collective futures unless they developed their reflexive capacities (Archer et al., 1998). Increased reflexivity would be necessary to actively participate in a globalised world.

Archer proposed, defined and developed her theory and modes of reflexivity in four key texts. In Being Human: the problem of agency (Archer, 2000), Archer laid out her disagreement with humanist's emphasis on social explanation rather than the encounter of the individual and the collective. She also rejected reliance on semiotics to explain social relationships. Instead, she proposed agency as a precondition of all human activity where a person would act on their concerns and commitments regardless of wider social change (Archer, 2000). In the first book of her trilogy - Structure, Agency and the internal Conversation (Archer, 2003), Archer addressed the lack of consensus on the nature and place of structures and agency. She argued that each individual has the personal capacity to reflect on themselves and their interests in relation to their social circumstances. She proposed this reflection taking place as an internal conversation with a dominant reflexivity mode. She defined three reflexivity modes which provided indications of agency as individuals encounter new structural forms. Archer then documented an emergent methodological approach to test her propositions.

In Making our Way through the World, Archer continued to develop and lay out her explanation of reflexivity and its characteristics - to fill the gap in sociological 
understanding of reflexivity, an "under explored", "under theorised" and "undervalued" social process (Archer, 2007). She posed that to be reflexive requires a sense of self and ingenuity to respond to change. In a shifting world, existing norms do not provide suitable reference points for some people to act on. Archer then tested these propositions on a segment of British social class that she was familiar with - educated university students and staff.

In the final book of her trilogy, The Reflexive Imperative in Late Modernity (Archer, 2012), Archer worked with the thesis that reflexivity is only seen in the "most developed parts of the world" (p. 1). This builds on her premise "it is social deconstruction that enables reflexive, narrative, serial and kaleidoscopic self-reconstructions by newly 'individualized' people" (Archer, 2012, p. 3). She presents this twenty-first century group of individualised people as the result of morphogenetic changes that have occurred in the United Kingdom since the 1980s. Overall, the trilogy emphasised the presence of internal or inner conversation, internal or inner dialogue, inner deliberation, internalised considerations, contemplation, or consideration inside one's head. Internal conversation is seen as the 'interplay' between structure and agency, a mediation process. It takes place within the privacy of the mind and is the key to understanding reflexivity. It is active, personal and deliberates on concerns and values (Archer, 2003, 2007, 2012). The key characteristics are it is interior, is subjectively directed and has an effect, i.e. causality. These characteristics are reflected at different levels in her modes of reflexivity.

\subsubsection{Modes of reflexivity}

Central to Archer's argument was that a changing or morphogenetic society has taken the place of morphostatic society as globalisation and technology have advanced. The stability or cultural reproduction of society provided 'contextual continuity' for individuals as they knew their place and roles in society. In a changing society, different forms of knowledge are passed between nations and people, and novel situations are experienced by individuals leading to 'contextual discontinuity' as people are distanced from the traditions of society (Archer, 2003, 2007). As a consequence, people have had to become more educated, flexible in thinking and are moving to meta-reflexivity, the peak of consciousness (Archer, 2012). Archer identified three main forms or modes of reflexivity in her typology, progressing in consciousness from communicative reflexivity to autonomous reflexivity and peaking at meta-reflexivity (Archer, 2003, 2007). A fourth - fractured or displaced reflexivity - was put forward (Archer, 2007) and explored in Archer (2012). Archer (2007) described ten mental activities of internal conversations that typified each mode, with the activities occurring at different levels of intensity. If 
university students are progressing towards meta-reflexivity as a result their education, this should be apparent in their shifts in mental activities and mode of reflexivity over an extended period of study.

\section{Communicative Reflexivity}

Communicative reflexivity is seen in relational people who participate in external dialogue in preference to the solitary nature of internal conversation. They are relational people who seek to check-out and validate their interpretations with their social peers. They want to confirm their viewpoints, choices and decisions are in keeping with those of their social groups and circles. They place a high degree of trust in the people with whom they engage in 'thought and talk processes', often family members. Their own views, or their dialogical partner's views, may be accepted or rejected as they work to find a shared understanding. The quality of relationships within their micro-world or natal/childhood context is important as the individual's concerns can be understood by their peers. Talking with others is for the purposes of completing and confirming thoughts already held, before following a course of action (Archer, 2003, 2007). Those who practice communicative reflexivity may develop new ideas and social networks as a result of new thinking, or their current thinking will be refined and reinforced (Archer, 2003). Agents would rather talk, consequently the mental activities of communicative reflexivity are reduced or limited. Plans are only made for the short term.

Employment, age and occupation do not appear to be critical but contextual continuity being known and belonging with similars and familiars - does (Archer, 2007). A sample $(n=10)$ of Archer's (2003) communicative reflexives from 128 case studies (which intentionally excluded university students and staff) included an equal mix of males and females aged from 18 to 69 years. Occupations included the unemployed, those retired from low skilled jobs and a retired teacher, a senior scientist and a banking manager. They demonstrated that the enablements and constraints of their reliance on their known group were not a focus of decision making, but they were involved in voluntary activities which addressed the concerns of their group and built the inter-personal relationship. They did not pursue their own advancement outside of their group. In another 2003 study of university students, Archer found family and close childhood friends were the most important informants for the communicative reflexives. Although surrounded by friends studying at university, it was the people the student grew up with and with whom they shared a common background that they discussed their lives (Archer, 2012). 
The concerns of those exhibiting communicative reflexivity features are refined and reinforced within their social networks. The purpose of holding the conversations is to reduce the load and extent of internal conversations. Their internal conversation may flow into the external world. This group likes the security of working with known relationships and contexts and this assists in maintaining the status quo. Those engaging in communicative reflexivity are "working at staying put" (Archer, 2007, p. 158).

\section{Autonomous Reflexivity}

Individuals demonstrating autonomous reflexivity have self-contained internal conversations which lead to direct action. They rely on their own mental resources, displaying confidence in their own ideas. They may consult experts for independent information but feel no obligation to accept their views (Archer, 2003). Their inner dialogue is comprehensive and encompassing. No other person is required. If constraints are identified they will work their way around them as their concerns and priorities are their business (Archer, 2003). Unlike the thought and talk processes of communicative reflexivity, people working in the autonomous reflexivity mode spend great periods of time in their inner dialogue considering topics, revisiting them, looking at them from multiple directions, exploring all the options. Even if they cannot reach a resolution, they feel no need to discuss their ideas with others.

A key characteristic is self-reliance. For some, it is simply that their natal contexts do not offer sufficient possibilities for the type of projects they wish to engage in and they move on from those who they might have consulted, had they wished to do so (Archer, 2007). There is no link between age, occupation or gender, but Archer (2012) found young "autonomous reflexives" often had broken or difficult childhood backgrounds where independence had been forced upon them, or they had chosen to move beyond the experiences of those around them (Archer, 2007). Their natal backgrounds did not provide stable relationships, normative traditions nor values they wished to follow. Contextual discontinuity occurs as the lack of connections means autonomous individuals can experiment in their thinking and actions without worrying about others. They are the "masters of contingency" (Archer, 2007, p. 286) and projects may be "unusual, innovative or considered to be risky" (p. 287). They spend time mulling-over often unrelated ideas only to return with certainty about a decision. Decisions are made and actions are well-planned towards outcomes.

Once a decision is made, they will execute their project to a high standard. If the outcome is not right, the process will be revisited. They are self-contained rather than concerned 
about others' opinions. They can position themselves both objectively and subjectively in social contexts and activities, working strategically. Archer also found this group represents the single-minded approach of those who are career-orientated, establishing their careers, or income focused, who limit social distractions and relationships as they pursue their goals (Archer, 2012). Relationships will be confined to partners, close family and children. They see their responsibility as citizens is in keeping social order. In this manner, they are making their own way through the world (Archer, 2007).

\section{Meta-Reflexivity}

Meta-reflexivity is seen in agents whose internal deliberations are critical of their own and others effective action in society. "Far from the social order being internalized or normalized, it is peculiarly problematized" (Archer, 2012, p. 207) as they are open to following the logic of change. They are aware of their inner dialogue being continuous, intense, or difficult to turn off (Archer, 2003, 2007). They are socially aware of circumstances which might hinder or advance ideas in their self-evaluation. They attempt to balance actions of self-interest against social good, considering and reconsidering possibilities. They hold strong ideals and will work around constraints or enablements to live out their ideal, foregoing social norms. They enjoy the situated logic in exchanges of ideas and have "an exploratory outlook towards the social" (Archer, 2012, p. 208).

Holding onto and wanting to act on their values is typical of meta-reflexive thinking and behaviours. Some will hold the values and concerns of their family and their upbringing while others are looking to develop their own priorities and social advantages of new contexts (Archer, 2012). Archer describes these individuals as having intense emotions over their ability or inability to act on their concerns, feeling that they are letting society down if they cannot make a difference (Archer, 2007). In their deliberations, they are looking for opportunities to act. They may disregard constraints but recognise the limitations of external circumstances on their internal decision making. Young individuals demonstrating meta-reflexive characteristics can appear critically detached from their natal backgrounds as they look to advance on their parents' circumstances.

Older individuals with meta-reflexivity features will be looking at ways to work on their dreams. When they encounter constraints that restrict or stop them following their goals they experience "contextual incongruity", where they cannot "idealise their ideals" (Archer, 2007, p. 244). They will often feel frustration at matters which are beyond their control. Archer describes the example of one person who requalified and moved 
sideways through multiple jobs, initially to provide an income and stability for family, but later to realise his dreams of helping people in deprived areas (Archer, 2007).

It is this value-orientation and their commitment to values which sets those manifesting meta-reflexive qualities apart from the other two modes of reflexivity. The sources of those values may be many and varied and their biggest concern is how to marry their commitments to their values with their careers. Their commitments may hold them back from advancing other areas such as financial advantage (Archer, 2007)

In seeking confirmation of her reflexivity modes, Archer found some individuals who did not clearly fit into these three modes (Archer, 2007). They appeared to be traumatically affected by some events or the immediate needs of planning for life and struggled with decision-making beyond the practice (Archer, 2012). She developed another type: fractured reflexivity.

\section{Fractured Reflexivity}

Archer defines those with the attributes of fractured or expressive reflexivity as people who as agents are not able to use their personal powers to create and act on projects, or to monitor "both self and society in the pursuit of their design" (Archer, 2003, p. 298). This is because they either do not believe they have the power to act or some other process or circumstance has intervened leaving them with reduced or no power, an "impeded reflexive" (Archer, 2012, p. 252). In this powerless state, they struggle to deal subjectively with the external environment, which in turn causes them further emotional distress and disorientation (Archer, 2003). Their internal conversation does not provide them with guidance; it can lead to emotional distress and uncertainty about their own goals and priorities.

Archer found three subgroups of fractured reflexivity which included those who had impeded reflexivity or were passive as agents due to circumstances (Archer, 2007). Archer termed these "displaced reflexives", as she imagined that should their situation change they would use a wider range of reflexive practice (Archer, 2012, p. 251). Those showing "impeded reflexive[s]" or under-developed reflexivity traits did not engage in thinking about themselves and society. They tended towards one mental activity of inner conversation, struggling to engage in the process of talking about their reflexivity (Archer, 2007). They struggled to relive their experiences, using phrases like "I don't know", “I worry a lot", "I want to get everything right", "I'm not confident", "I didn't have a good life", which intensifies their confusion and clarity of their concerns (Archer, 2007, 2012). The 
third group were those who seemed to have little or no internal conversation or self-talk, "near non-reflexive" (Archer, 2012, p. 252).

Archer used the case of Julie to demonstrate displaced fractured reflexivity. Growing up, Julie's parents held strongly different concerns. This sometimes generated a tense environment which undermined Julie's confidence and left her worried about others' views of her. In her first year away at university, Julie idealised the positive aspects of her relationships with family and close friends with her new acquaintances, marks of communicative reflexivity. In her second year, when flatmates argued with each other, she became withdrawn, less confident and passive - characteristics of fractured reflexivity. However, in her third year after she moved closer to home and reconnected with her family and friends, she once again showed communicative reflexive characteristics, and reaffirmed the positive aspects of her upbringing (Archer, 2012).

Archer believed with society morphogenesis, or rapid change, there would be an increase in the numbers of people with experiences of fractured reflexivity, as the diversity of values and concerns an individual would confront would be disruptive.

In this section, I have presented the foundations of Archer's concept of reflexivity in the relationship of structures, agents and agency. I also presented key debates around Archer's theory, agreeing with other commentators that in premising a strong ontological position on the nature of reality Archer limits the range of perspectives on what reality means, how it is known, and how that knowledge influences reflexive development and personal agency. Commentators suggest including aspects of social and cultural construction would bring more balance to Archer's morphogenetic and reflexive approach.

\subsection{Internal conversation and determining internal conversation}

In this section I present understandings of the internalised ways in which people consider the things they hold important against the widening range of views encountered in a technologically enhanced mobile world.

The processes of human reflexivity and agency are reliant on the presence of internal conversation, self-talk, inner dialogue or internal deliberation (Archer, 2007). Archer presents reflexivity as the means through which agents encounter the structures of social and cultural conditioning, consider them internally, and are able to act based on their concerns (Archer, 2000, 2003, 2007, 2008, 2012). The properties of structures and culture have power attributed to them by society, which influences the personal power 
individuals believe they have. Through the form of an internal conversation, people consider objects that concern them or society (Archer, 2007).

The ability to have important internal conversations (as opposed to everyday mundane thinking e.g., what shall I eat tonight?) is an "emergent personal power of individuals" as the embodied mind responds to the questions it puts to itself (Archer, 2007, p. 3). Inner dialogue is an active private conversation where ultimate concerns are deliberated (Farrugia \& Woodman, 2015). In internal conversation, people define and clarify their beliefs, values and goals and consider their social circumstances. "Internal dialogue is the practice through which we 'make up our minds' by questioning ourselves, clarifying our beliefs and inclination, diagnosing our situations, deliberating about our concerns and defining our own projects" (Archer, 2003, p. 103). It shapes the kind of modus vivendi that we want to have.

The life of our minds is always to some extent, taken up with the life we want to live. Because of human life, it will mainly be lived socially and many (although not all) of our concerns will be explicitly social in kind (Archer, 2012, p. 14).

Individuals who regulate the relationships between themselves and their society as they consider and develop projects based on their concerns, are active agents. Those whose personal powers are obstructed are passive agents (Archer, 2003; Caetano, 2017b). A difficulty for researchers wishing to observe internal dialogue lies in its nature - it is private, only observable to the individual. It is this challenge that Archer addresses in empirically capturing inner conversations through asking people what is going on in their heads: what they are saying to themselves and what it is about.

Internal conversation plays a part in an individual's construction and reconstruction of their thinking about their social world as they reflect on values, routines and habits in ongoing secondary socialisation (Berger \& Luckmann, 1966). Archer's use of the word conversation suggests a form of dialogue or use of language in inner deliberations, but it is a bigger "inner life" including free-form thoughts, imagery, intentional comparisons of ideas and revisiting discussions (Archer, 2007), in which the "intra-active process[es] ... of deliberation, evaluation and selection" occur (Archer, 2003, p. 63). Others, King (2010), Caetano (2015a) and Chalari (2013), claim Archer ignores the importance of the social and external exchanges on reflexivity. Caetano and Chalari make a clear distinction between external conversation (the verbal or expressed inter-action a person has with one or more individuals) and internal conversation (the non-vocal intra-action 
the individual has with themselves). However, Archer (2012) saw the two as integrated, albeit somewhat distanced:

To engage in inner dialogue is to activate our personal powers, but that does not make any of us individualistic monads. We all receive and use external information, we all engage in external as well as internal conversation and, above all, being human refers to a quintessentially relational being. Our human relations and the relationality between them form part of both our internal and external conversations". (Archer, 2012, p. 15)

Burkitt (2010) attempts to distinguish between inner conversation and the "dialogical unconscious" - listening to the voice of others in one's mind, in a manner which is semiautonomous, but conscious of the other within oneself. Inner conversation is described as a hidden or implicit voice, a micro-dialogue, to some extent unconscious or not recognised as the self-as-knower. The voice of the other can have an impact or influence upon the self. In clarifying what internal conversation is, Chalari (2007) places the emphasis on the "inner, private and intra-actional process" which may be non-linguistic and with an internalised response (Chalari, 2013, p. 69). Caetano suggests three main "elements: they are interior, in that they occur quietly in a mental space, are not directly observable from the exterior, and do not necessarily have any behavioural manifestation" (2017b, p. 68).

To demonstrate that internal conversation was observable, Archer interviewed people about the ideas they consider in their internal conversations and how they acted to determine if they are active or passive agents (Archer, 2003, 2007, 2012). Her premise was that people are involved in internal dialogue with variability and more intensity at different times in their daily lives. However, it is the quality of the conversation which differentiates internal deliberation from mundane or random thoughts, elevated through its dependence on individuals' "concerns" and "projects" which are important to them (Archer, 2007).

\subsubsection{Determining internal conversation}

Archer developed tools for drawing out and classifying internal conversations using deductive methods across a number of pilot and full studies. Using small scale studies of purposefully selected groups within the United Kingdom (UK) at the turn of the century (reported in Archer, 2003, 2007, 2012), Archer wanted to see if three types of reflexivity - communicative, autonomous and meta-reflexivity (Chapter 2.2.6) - were representative of people's agency. 
In the first study, propositions on the relationship between structures and agents were tested to see if the person's emergent personal powers were observable. A small "diverse" group of twenty interviewees, Archer's friends and personal contacts, were interviewed in-depth to establish if the reflexivity modes proposed covered the processes displayed by participants and measured variations in the intensity of internal conversations (Archer, 2003). Questions were presented in two parts.

The first set of questions covered reactions and thoughts about the notion of internal conversation. Interviewees were asked about their use of 10 mental activities derived from an earlier, but undocumented, pilot study (Archer, 2003). These activities were: planning (the day, the week, the future), rehearsing (practicing what you will say or do), mulling-over (focusing on a problem, situation or relationship), imagining (the future, including speculating), deciding (debating what to do, the best option), clarifying (sorting out what you think about an issue, person or problem), imaginary conversations (held with people you know or are known to you), budgeting (affordability in terms of money, time or effort), re-living (some period, event or relationship), and prioritising (working out what matters most, next, or at all) (Archer, 2003, p. 161). The degree of use of mental activities define the characteristics of the reflexivity modes (Chapter 2.2.6). Part two asked participants about their concerns (matters important in their life) and life-projects related to their "social class, status and power" to see how they internally deliberated about their future (Archer, 2003, p. 162). In her study, Archer found a fourth mode fractured reflexivity, where five individuals were unable to exercise their power or design and pursue projects (Archer, 2003).

Archer also found people's dispositions towards their experiences and actions were influenced by their view towards their wider social networks, personal contexts, and concerns over time. She defined three "different stances towards society and its constraints and enablements: the evasive, the strategic and the subversive" indicating the participant's ability to control interactions between them and their society (Archer, 2003, p. 342).

A subsequent quantitative and qualitative study, of a single social class in Coventry (UK), looked at 128 individuals' considerations of their social mobility in their internal conversations (Archer, 2007). A smaller group were questioned about the use of mental activities. "Quantitatively the vast majority of the forty-six subjects interviewed ... agreed that they engaged in at least half of those activities" with a few indicating they engaged in all ten (Archer, 2007, p. 91). Archer then trialled a 24-question Likert scale, multidimensional questionnaire (ICONI) designed around the mental activities found in her 
2003 study, in an attempt to find a quick and easy tool, to consistently identify her reflexive types (Archer, 2007, 2008). The ICONI questionnaire was administered to 64 university employees, and after refinement of the questions, to 130 first-year university sociology students. Findings suggested patterns of mental activities towards constraints depended on the structures participants considered (Archer, 2007). However, ICONIC did not appear to adequately discriminate between the modes.

A follow-up three year quantitative and qualitative study showed strong variability in reflexivity modes between groups and across disciplines (Archer, 2012). The longitudinal study of 126 undergraduate sociology degree students by Archer's PhD student Mark Carrigan, tested for consistency in the characteristics of the modes used represent internal conversations. The foci of these students' concerns were differentiated as: family, friend and social life for those with communicative reflexivity traits; careers and performance skills for autonomous reflexivity traits; with meta-reflexivity traits focused on socio-political and faith-based moral causes and issues (Table 2.1). Those with fractured reflexivity characteristics were just concerned with the here-and-now of daily existence or survival. Archer linked fractured reflexivity to lower educational levels and lower socioeconomic groups, rather than other social circumstances. She also attributed the reason for $49 \%$ of students showing no change in reflexivity modes over time was due to their stable socio-economic backgrounds (Archer, 2007, 2012).

Table 2.1 Primary concerns of University of Warwick students (adapted from Archer, 2012)

\begin{tabular}{|l|l|l|}
\hline Reflexivity Mode & Primary concerns & \multicolumn{1}{c|}{ Foci } \\
\hline Communicative & $\begin{array}{l}\text { Interpersonal } \\
\text { relations }\end{array}$ & $\begin{array}{l}\text { Family and friends, family, friend, } \\
\text { relationship (singular), university social } \\
\text { life }\end{array}$ \\
\hline Autonomous & $\begin{array}{l}\text { Careers/performative } \\
\text { skills }\end{array}$ & $\begin{array}{l}\text { University work, money, performative } \\
\text { interests (sport, music, drama) }\end{array}$ \\
\hline Meta-reflexivity & $\begin{array}{l}\text { Moral causes and } \\
\text { issues }\end{array}$ & $\begin{array}{l}\text { Socio-political causes (including social } \\
\text { movements, faith) }\end{array}$ \\
\hline Fractured & Immediate life & \\
\hline
\end{tabular}

Evident in this narrow range of studies is that Archer's reflexivity modes and tools to determine modes were insufficiently developed and tested to draw conclusions on their use to determine changes in agency in stable social groups in the UK. While a number of studies have directly applied these approaches to groups within single cultures and in response to particular events, no studies considering diverse cultures or contexts have been reported. However, Archer's mental activities, reflexivity modes and qualitative 
methods can provide a platform for further investigation of structure-agency considerations in a wider range of situations.

The approaches used in studies by Archer to determine internal conversation and reflexivity modes have been outlined. In trials, the various tools asked individuals what issues were most important in their lives. The grouping of concerns was distinct to each of her reflexive modes. A discussion of the differentiation of values, concerns and how projects are formed is presented in the next section.

\subsection{Values, concerns and projects}

\subsubsection{Values and concerns}

In Archer's work, traditional knowledge and beliefs and the values attributed to them by a social group or class are the glue which hold society together (Archer, 2003). Accepted norms of behaviours and expectations are integrated through socialisation and maintained by social stability. However, the breakdown of traditional social structures and their associated beliefs, and the entry of new knowledge and social encounters provides the opportunity for reconsideration of what is important and has value (Archer, 2007). Values become contested and more flexible, reconsidered by the individual rather than imparted by society. In Archer's structure-agency-agents relationship, concerns are what people value or care about the most (Archer, 2012; Caetano, 2019). Values are concerns connected to our perception or sense of self and our preparedness to commit at some level to them and to determine action (Caetano, 2019). Values are wide-ranging including fairness, justice, reciprocity (Ahier et al., 2003), meaningfulness, satisfaction in life, and study-life balance (Coetzee \& Oosthuizen, 2012), and are open to multiple interpretations.

Archer believed personal identity was developed through individuals monitoring and prioritising their values and concerns (Archer, 2003, p. 41).

[T]o have a personal identity is defined by our constellation of concerns and to have a concern is necessarily to be concerned about it. .... The life of our minds is always, to some extent taken up with the life we want to live. ...The prime social task of our reflexivity is to outline, ..., the kind of modus vivendi we would find satisfying and sustainable within society. What we are attempting to accomplish is to marry our concerns to a life that allows their realization, a way of life about which we can be wholehearted, investing ourselves in it with each personifying its requirements in our own and unique 
manner. Hence, we gain and maintain some governance over our own lives. This is a supremely reflexive task, entailing 'strong evaluation' of our social contexts in light of our concerns and adjusting these concerns in the light of our circumstances (Archer, 2012, pp. 14-15).

Concerns emerge from patterns of commitments in internal conversations as people reflect on their values. As individuals move through the world, they encounter different values. They must exert what Archer (2003) calls the "first causal power" of reflexivity in deliberating if they want to incorporate these features into their own schema.

Individuals prioritise their concerns and find a means to accommodate concerns that are subordinated (Archer, 2003) stated. They separate out their "first order concerns" (Archer, 2003, p. 27) - those most influential in defining their life activities - from those considered least important. This individualisation of priorities plays a part in defining individuals' identities in the way in which they work their concerns together against the values they wish to live out (Archer, 2003). Concerns are balanced or dove-tailed in light of immediate daily responsibilities, the interrelationships in micro-worlds of personal contexts (family, friends) and social contexts (careers and work). However, Archer argued that in late modernity's globalised and diverse environments these concerns may be balanced against a wider range of contexts, particularly in meta-reflexivity.

The forces of globalisation have brought another layer of more intricate concerns into people's lives. Individuals now consider their place, contribution to and responsibilities in structural realms wider than those previously considered, and in situations in which they have limited ability to act. However, Archer maintained that for some contextual continuity - geographic stability and continuity in schooling and social contexts, stable family relationships, and the availability of work in natal contexts - would sustain rather than disrupt their reflexive practices. These people would continue to consult family and close friends to discuss their concerns, and to confirm their thinking and actions fit within their social groups through "thought and talk" (Archer, 2007, pp. 145-146). The closeness of the connection between current contexts and structures of wider social networks (e.g., economic) would determine whether or not people acted on their concerns to create projects.

\subsubsection{Projects}

As identified in the last section, projects are intentional courses of action triggered by a concern (Caetano, 2019). The pursuit and implementation of a project within social environments depends on how much power individuals feel they have to make the 
change. Projects have structural or cultural properties and the power these properties have over the individual are "activated by agents" in considering them (Archer, 2007, p. 12). Using the command people have to consider what to do in a particular situation, individuals consider their values and define projects in keeping with values, but must do so by considering the enablements and constraints between themselves and the social world - the "micro-macro link" (Archer, 2003, p. 343). In doing so, they recognise the potential for different courses of action.

Archer's position was that individuals are not free to act, unrestrainedly. They are constrained or enabled by the structures, social and cultural contexts (or social conditions (Caetano, 2019) in which their projects sit. Structures can have power over choice and ability to act only if the individual sees there is agreement or difference between the structure and their project (Archer, 2007). Once identified, the person can then take a "strategic", "subversive" or "evasive" stance or approach towards their project in dealing with structural features. In doing so they demonstrate they are "active agents" (Archer, 2003, pp. 342-343). Despite this seemingly encompassing position, Archer's focus on rapid change meant she was only interested in an individual's reflexive experience within their immediate social and cultural contexts. While this may apply to isolated and mobile individuals living in highly differentiated urban environments, other people continue to live in areas that are more socially stable or linked together through culture or occupation (for example, suburbs with a particular ethnic population or farming communities). All people live in a rapidly changing world but how each person experiences it differs.

The forming of a strategy, development and activation of a project is affected by three things: an individuals' agential capability, belief in their ability to exercise their personal power to accomplish goals, and access to the resources available to them (Archer, 2007, 2012). As indicated above, enablements or constraints do not have to be enabled or disarmed, acknowledgment of their existence is sufficient (Archer, 2007). While Archer focused on the activation of projects in the present, other factors may come into play. In referencing their past socialisation and experiences, individuals can be open to a range of personal projects having learnt not only what they are capable of doing, but also what they are interested in achieving (Caetano, 2019). By taking a dualist approach Archer believed that it was possible to observe the power structures currently have over individuals as they consider the enablements and constraints of those structures, their options and actions. However, structural power and their enablements and constraints can precede the immediate structure-agent relationship. Therefore, to understand the 
on-going effect of structures on projects it is worth considering how projects evolve over time and the historical influences on their development.

It is at this point that Archer's proposition blurred with Bourdieu's field of possibilities. The relationship between concerns and projects is interwoven (Archer, 2003). Some projects take on a more permanent nature, but a person's concerns are generally dynamic and are often reworked and readapted, even if only partially (Caetano, 2019). Archer was not alone in considering the relationship between concerns and projects. Projects are not only based on an individual's agential abilities and reflexive competencies, as Bourdieu (1984) frames them within a field of possibilities delimited by an individual's sphere of action and their social and cultural capital. Experiences, prior opportunities and limitations within a subject's life contexts play a part in defining the possible options. Available resources and means, and possibilities and difficulties arising from people's backgrounds, can be consciously identified (Caetano, 2019), but can also be dependent on other personal characteristics and self-belief. Resources - time, finances and energy mentioned by Archer (2003) in the mental activity of budgeting - can affect choices. This is an area of the structure-agency interaction which requires further consideration.

Others, such as Caetano (2018) and Adams (2003), believed that Archer's work underplays the temporal dimensions, specific features and conditions which affect consideration of projects. Caetano (2018) argued that strategies to pursue personal goals take time to develop and become more concrete with time. The closer the project is to implementation, the clearer a person's understanding of the contexts and resources needed, and actions that must occur for a project to succeed becomes. Projects can

change over time in line with changes in the central concerns of individuals and in their prioritisation. Some projects take on a more permanent nature, but a subject's concerns are generally very dynamic and are often reworked and readapted, even if only partially (Caetano, 2019, p. 6).

Elster (2017), Adams (2003) and Tsekeris and Lydaki (2011) also noted the knowledge a person already had gained through developing projects is referred to and influences current reflexive thought.

In studying biographical life courses, Caetano (2015a) and Mrozowicki (2011) found participants reflexively filtered the potential for their projects by their social setting and by the responses of others to their projects. Projects were reworked, adjusted, negotiated, or suspended depending on how open or close the social contexts were considered to be by the individual (Caetano, 2017a, 2019). Consistent with Archer's (2007) social 
mobility studies, Caetano found respondents with higher levels of education and more socioeconomic resources designed more elaborate projects, had greater confidence, and autonomy in their ability to act on their circumstances to achieve their plans. Respondents from more disadvantaged socioeconomic positions and limited resources struggled to find alternative ways to achieve their goals and gave up or became fatalistic (Caetano, 2019). Not all projects have the same value to the individual. Projects can succeed, fail or lead to unexpected results, are interrupted by contextual changes, or may not able to be implemented.

Caetano (2019) also challenged Archer's and Giddens's assumptions that the reflexive individual is emancipated enough to use their personal powers to form projects which bring about change. Wider consideration of the social conditions (contexts and circumstances leading to change) making reflexive social action possible is needed, rather than just assuming that robust reflexivity always produces action (Caetano, 2019). Some people may plan towards aspirational projects which are outside of their current capacity, where congruence or incongruence between projects and immediate resources may not be as important. Concurrently studying reflexivity, changes in context, structures and resources helps the researcher to see how changes in people's values, priorities, and concerns affects project formation and realisation.

Project outcomes cannot be guaranteed. In her 2015 study, Caetano found changes in social conditions and personal commitments may result in a change in projects. People also lost interest in achieving the goal or their external circumstances changed (Caetano, 2019). Caetano's findings were consistent with Archer's (2007) finding that project implementation may appear to fail when projects do not lead to expected outcomes. Mismatches in expectations or resources means the proposal must be adjusted, reworked or abandoned.

There is also the possibility, despite reflexivity's central role in moving the person towards action, that "paralysis of action" may occur (Caetano, 2019, p. 8). The individual may feel incapable of making a decision. They may decide nothing further is to be done about a concern or they abandon the project. Paralysis of action may result from mismatches between personal characteristics and the project context, or occur when strategies for action are not fully developed. Internal conflicts in thinking on the issue, roles or reference points and disorientation by overthinking can occur in those who spend a lot of time in reflexive thinking. 
The concept of projects is critical to Archer's structure-agency interaction, yet as presented above external and internal social conditions and experiences can influence the outcome and therefore perceptions of agency. Where reflexivity modes are dependent on the achievement of projects, underlying conditions and influences also need to be considered.

\subsection{Criticism of Archer's reflexivity approach}

As indicated earlier, Archer's work has been scrutinised by a number of sociologists from different ontological positions. The main criticism of Archer's work on reflexivity accused her of the very conflation (privileging of power) she was trying to address - an overemphasis of individual autonomy (Akram \& Hogan, 2015; Caetano, 2015a). It appeared that Archer's reflexivity and Giddens's structuration swung so far away from the power of structures over agents that they now isolated the individual from their social contexts. King (2010) described Archer's agent as "an isolated figure [wandering], engaged in private conversation" (p. 257). Instead, King proposed social theory needs to move from thinking vertically about structure and individual agents to thinking horizontally to include investigation of how social networks and structures operate across multiple participants, who have different thoughts about structures as they interact and work with each other. While Archer argued that traditional social networks have broken down, there is a need to consider how new networks and relationships influence reflexivity and if old influences demonstrate the influence of habitus or something completely different.

\subsubsection{Archer's reflexivity - criticisms and responses}

A major criticism of Archer's work on reflexivity and internal conversation is that it lacked sufficient reference to the place of socio-cultural background (Bourdieu's habitus and life histories) and its influences on the self in internal conversation (Adams, 2003; Akram \& Hogan, 2015; Caetano, 2015a; Elder-Vass, 2007). Archer proposed that her analytical approach treats structures and agents separately allowing for greater understanding of interactions and outcomes at the individual level. Archer saw Bourdieu's view of habitus as a fixed system of ways, beliefs and values based in past experiences no longer appropriate in postmodernity. Under Bourdieu, individuals' agency is constrained when they "feel like the social construct is natural and therefore unchangeable" (Archer, 2007, 
p. 314). Rather, Archer argued for the recognition of the presence of personal habits and a more flexible view of social conditions.

Archer's reading of Bourdieu is seen as too narrow (Akram \& Hogan, 2015; Farrugia \& Woodman, 2015) leaving a lack of depth in her empirical studies of reflexivity and internal conversation (Caetano, 2014; Elster, 2017). Without reference to the embedded nature of habitus in a person's consciousness and responses, an important aspect of the mechanisms of reflexivity is lost (Elster, 2017). In particular, Gronow (2008) argued that habitus overrides reflexive thinking particularly in stressful times. "Reflexive deliberation is subordinate to habitual dispositions because the former is usually present in situations of crisis, where habitual action is the normal state of affairs" (p. 243). Caetano (2014) points to previous discussions on the absence of the role of resources in decision-making in the reflexive modernisation and individualization thesis. She continued on to argue that the use of social class to distinguish resources available in reflexivity would be better replaced with other considerations such as social inequalities, gender, lifestyles and cultural practices. Dismissing habitus outright leaves a gap in understanding pre-existing influences of the social on the individual's responses to new situations or ideas.

Many suggestions have been made for some form of integration of habitus into Archer's theory to account for other biographical, past and present influences on agency (Biesta \& Tedder, 2007; Emirbayer \& Mische, 1998). Akram and Hogan (2015) argued for this on the basis that "habitus is written deeply within us by multiple, layered, intersecting and at times conflicting social processes" ( $p$ 608-9). Agents do not have control over the processes. If Bourdieu's and Archer's works are treated as "emergent" theory and habitus as an evolving characteristic of life, then human action is the result of the continuous interplay between dispositions and reflexivity (Caetano, 2019, p. 102; ElderVass, 2007). Less conspicuous evidence of action, than Archer was looking for, may then be evident (Caetano, 2014). Archer (2012) countered Elder-Vass's (2007) suggestion claiming in modernity there is new social order. She suggested Elder-Vass characterised a reflexive personal identity only achieved at maturity and not by everyone. Archer had found that under morphogenesis young people demonstrated limited reflexivity as, even when they had the choice, they had not connected to habitus sufficiently to acquire its reference points (Archer, 2012).

Further tensions arose out of Archer's dismissal of social construction, for giving too much power to the individual's contribution to understandings of reality, and Mead's (1934) presentation of 'l', 'me', and 'you' in identity construction (Archer, 2000). She regarded social construction's views of the self, personal and social identity developing 
out of on-going socialisation experiences (Berger \& Luckmann, 1966; Elder-Vass, 2012; Gergen, 2011) as being too fixed or stable (Archer, 2003, 2012). As with Archer's view of Bourdieu, this also appears to be a narrow interpretation of Mead and social construction. Berger and Luckmann (1966) saw internal conversation as a part of society's conscious socialisation processes, with individuals as social beings from birth who become increasingly aware of their place in society. Emotional and affective belonging and ideas, values and attitudes of immediate people and environment are internalised into identity in primary socialisation during childhood (Mead, 1934). However, through secondary socialisation, the "process that inducts an already socialized individual into new sectors" of society continues, notions of identity and belonging change throughout life (Berger \& Luckmann, 1966, p. 150). Gronow (2008) considered that Archer's anti-Mead position, as part of her argument against social construction, leaves an "under socialised picture of self-hood and agency" (p.244). In doing so, Archer's personal identity is fixed to a point in time ignoring the input of changes between contexts at different points in life. This appears to be at odds with Archer's position of the requirement for individuals to develop the ability to reflexively respond in times of change. If the reference points of identity (e.g. family, work, nationhood) are undergoing change then identity changes. Consequently, there is a need to re-emphasise the importance of identity and changes of identity in structure-agency in times of social transformation.

Social construction theories place greater emphasis on socialisation and awareness of social processes in identity formation than does critical realism. On-going socialisation recognises the presence of Bourdieu's habitus, and elevates the importance of the changing and dynamics of social circumstances that people find themselves in, where different perspectives and ideas are shared. With Archer isolating individuals from their immediate and wider social contexts, she failed to recognise the subtleties of observable and unobservable structures which influence concepts of the self, priorities and thereby individual and joint concerns and projects (Elder-Vass, 2007; Farrugia \& Woodman, 2015; Mutch, 2004). Fellow critical realist, Mutch (2004) suggested Archer's emphasis on structure-agency would offer a more balanced understanding of individuals' reflexivity and agency if the situated nature of their social contexts and their processes is recognised.

Archer's approach towards agents is also considered too impersonal - objective and rational - ignoring the importance of feelings, emotions and moral consequences of reflexivity. Archer refers to ultimate concerns as sustaining internal conversation (2007), which links reflexivity "to our emotional commitments" (Akram \& Hogan, 2015, p. 607). 
Relational theorists Burkitt (2012) and Holmes (2010, 2015) argued that emotion is always present in our perception of ourselves, others, and the world around us. It affects engagement and interrelationships and therefore its presence in reflexivity should not be ignored. People are involved in many different relationships and understandings of structures at any one time and this affects how people respond. Social structures are never confronted as a single individual but in interpersonal or more impersonal relations which have their own power imbalances and therefore implications for agency (Burkitt, 2016). Instead, Burkitt (2016) suggested that using a relational or emotional reflexivity or social network approach (Elster, 2017; King, 2010) would consider attitudes and feelings of self, others and the world seen in social interactions. Expressed through self-feeling and self-reflection these can be recognised in dialogue as expressions of "I feel" "I felt" and "the feeling I" (Burkitt, 2012). However, Holmes (2010, 2015), cautions the researcher in that people may be unable to directly articulate the tangle of emotions present in their everyday interactions.

Archer, on the other hand, argued researchers need to be careful not to over emphasis socialisation. There is a difference between a psychological sense of self 'who I am' (personal identity) and a social concept of self - 'how I define myself' compared with others (social identity) (Kaufman, 2014; Mead, 1934; Stets \& Burke, 2012). For Archer, how the self is presented depends on the language used and it is an epistemic fallacy to confuse knowledge and what really exists (Archer, 2003).

Discussion continues on the symbolic interaction role and the importance of language in reflexivity. Mutch (2004) and Kaufman (2014) reasoned there was a need to recognise the social linguistics of structures, language shapes the conversations a person has about their identity and limits the type of projects they can pursue. Self-knowledge and internal dialogue require the use of language. In reflexivity an individual puts questions to the self in order to find answers to those questions (Elster, 2017; Tsekeris \& Lydaki, 2011). Language is historically and contextually located as well as providing the means for conversation; habitus provides the basis for shared meanings (Susen, 2016). In other words, reflexivity is a complex dialogical interaction that requires some language or discourse analysis to interpret its processes (D'Cruz, Gillingham, \& Melendez, 2007). Adams (2003) and Beck et al. (2003) also acknowledge reflexivity as a very Eurocentric concept that makes assumptions about the need for reflexive thought to "shape and control civilizational development" (Susen, 2016, p. 51), an approach that may have limitations beyond this cultural pool. 


\subsubsection{Empirical studies using Archer's reflexivity approach}

The number of empirical studies applying Archer's structure-agency theory and methodology of reflexivity as internal conversation is growing (for example, Baker, 2019; Case, 2013, 2015; Dyke et al., 2012; Kahn, Everington, Kelm, Reid, \& Watkins, 2017; Matthews, 2017). Archer provided varying levels of detail of the steps in her methods and analysis in text (Archer, 2003) and in methodological appendices (Archer, 2007, 2012). Hence, studies using her work are either varied in, adaptive of, or restricted to, what she has described. Studies considering modes of reflexivity across age groups (Caetano, 2015b; Mrozowicki, 2011), and those with students in higher education or transitioning into training (see Bovill, 2012; Ellery \& Baxen, 2015; Kahn et al., 2017; Matthews, 2017), take two approaches - the application of existing methods (e.g. Baker, 2019; Hung \& Appleton, 2016), or adaptations and expansions of methods to develop modes specific to the research context (Caetano, 2015b; Case, 2015; Mrozowicki, 2011).

Studies vary in topics, data collection approaches and participant numbers and are limited to predominantly European monocultures including the United Kingdom and Italy. Topics include: UK students' reflexivity in relation to social processes, transitions, projects and concerns in UK continuing education choices (Baker, 2019; Bovill, 2012; Cieslik, 2006; Dismore, 2016; Dyke et al., 2012), transitions between UK welfare structures (Barratt et al., 2019; Hung \& Appleton, 2016), international students' adjustments to UK university contexts (Matthews, 2017), Italian youth transitions to adulthood (Domecka, 2017), tutor professional development in medical disciplines (Kahn, 2013) and student engagement in online learning as the exercise of agency (Kahn et al., 2017). The exception to the European monocultures are studies from South Africa looking at socio-economic structures and cultural conditions that affect students' agency in university study (Case, 2013, 2015; Ellery \& Baxen, 2015; Luckett \& Luckett, 2009), and a recent study from Australia looking at vocational students' identity construction through reflexivity (Ogilvie, 2017). Two other more detailed, across age-group, biographical studies, studies look at participants' reflexive responses and agency in Poland (Mrozowicki, 2011) and Portugal (Caetano, 2015b, 2017b; Nico \& Caetano, 2015).

Qualitative and quantitative approaches are used for data collection and analysis; however, studies often do not specify if, or how, Archer's tools are used. Data collection methods include single interviews (Case, 2013, 2015; Hickey \& Austin, 2007), semistructured, in-depth interviews with thematic analysis (Chalari, 2007), multiple extended interviews (Caetano, 2015b; Hung \& Appleton, 2016), retrospective coached interviews 
(Cieslik, 2006), and reflective journals and questionnaires (Bovill, 2012; Hickey \& Austin, 2007). Rainford (2016) used auto ethnography to reflexively explore his doctoral experience of using new technology. The use of qualitative narrative or biographical interviewing limits participant numbers. Respondent numbers range from a single case (Ellery \& Baxen, 2015; Rainford, 2016), small groups of 5 -15 (Baker, 2019; Cieslik, 2006; Hung \& Appleton, 2016; Kahn et al., 2017; Matthews, 2017) and larger groups greater than 50 people (Bovill, 2012; Case, 2013; Luckett \& Luckett, 2009). Mrozowicki (2011) gathered biographical data from a large number $(n=166)$ of respondents in a mixed methods study of Polish workers responses to political change. The level of method and analysis detail is variable, dependent on the study scale, creating difficulties in interpreting and advancing understandings of internal conversation's role in reflexivity and agency.

Three empirical studies (Caetano, 2015b; Hung \& Appleton, 2016; Mrozowicki, 2011) and a thesis (Chalari, 2007) provide greater detail which allows for a closer examination of their findings. Hung and Appleton (2016) described the application of methods following Archer's (2007) approach. In the first extended interview, they asked nine purposefully selected participants (aged 19-24 years) for their thoughts on internal conversations and its mental activities. This required a high level of prompting to explain internal conversation and how it might occur. In the second interview, they asked about participants' concerns and plans for the future or life-projects. Huberman's interactive model and interpretive phenomenological analysis were used interactively and inductively. Hung and Appleton (2016) found their participants had varying levels of agency and reflexivity on moving from state care into training or work. Two of their three categories (emerging active agency, survival oriented reflexivity, or passive agency / fractured reflexivity) fit with Archer's work, while survival orientated agency was a new category (Hung \& Appleton, 2016).

In their study on postgraduate online learning students' engagement in their studies, Kahn et al. (2017) initially collected and analysed discussion board postings to access students' $(n=22)$ exercise of their agency in their concerns towards completing their qualification - their project. Follow-up interviews with eight purposively selected students (from four geographic regions) explored the nature of their reflexive practice on their study contexts (Kahn et al., 2017), rather than course content and its effects. The researchers found traits of several of Archer's reflexive modes present in all participants, with communicative reflexivity present in all but one, and no dominant modes present. They also found "significant overlap" between concerns in the communicative and 
autonomous reflexive modes. However, there was no discussion of external influences on or considered in the achievement of the project. Kahn et al. (2017) study provides some evidence of how higher education students reflexively consider their values, concerns and possibilities for acting. My study helps to extend the groundwork for approaches that can be taken in small-scale studies to consider the wider influences on online or distance learners' reflexive considerations of their agency.

\subsubsection{Adaptations to Archer's reflexivity and internal conversation}

Adaptations to Archer's reflexivity theory and internal conversation approaches and methods have been made in a small number of in-depth studies. Researchers have sought to elaborate and move beyond the limited range of critical theories and theory confirmation used by Archer to study social mobility in Britain to consider other sociological theories and cultural contexts.

\section{Chalari - inner and outer dialogue}

The connection between dialogues of the inner and outer person suggest that internal conversation is the means by which inner thoughts are moderated before being externalised (Chalari, 2007). In her thesis, Chalari reviewed and examined the theoretical arguments of internal and external conversations in the work of Archer, Giddens, Simmel and Goffman and the American pragmatists James, Peirce, Dewey and Mead. She proposed that an equal emphasis on both internal and external conversation, rather than foregrounding the inner as proposed by Archer, was needed (Chalari, 2007). In a later study, she collected 26 Greek adults' views of their responses to socio-political changes in Greece in semi-structured interviews and thematically analysed them (Chalari, 2013). Chalari captured and examined participants' internal conversation and external conversation - with external conversation being the "verbal interaction each person has with one or more individuals while internal conversation is non-vocal intra-action the individual has with self" (Chalari, 2013, p. 69). This work accounted for wider social interactions and external influences more than Archer's work and looked at internal resistance to events and changes in conditions, as an alternative to Archer's physical action outplaying of agency. Chalari found there were strong individual differences in the way internalised dialogue affected external triggers. The two forms of dialogue were interrelated and interconnected concepts, but it was the internal which mediated the balance between what is considered and what is spoken. This incorporation of the influence and interplay of both internal conversation and external conversation is an enhancement on Archer's reflexivity. 


\section{Mrozowicki - individual or community focus dialogue}

In his study on working-class individuals $(n=166)$ in industrial Poland, Mrozowicki (2011) looked at how they coped, adapted or resisted structural changes in post state-socialism systems. He considered how they used their diverse resources, culturally shaped beliefs, and their ability to reflexively define their life projects. Mrozowicki's study applied Archer's (2003) belief that people had evasive, strategic or subversives stances towards change. The central research question of the study looked at forms of collective ethos and individual reflexivity. Based on Archer's structure-agent-agency theory and Bourdieu's (1984) theory of habitus (ethos/character of beliefs and values), Mrozowicki used Schutze's (1983) biographical narrative interview and grounded theory methodology (Mrozowicki, 2011). He looked at the typical patterns workers used in their lifetime's stories to reinterpret the socio-political changes that had occurred. The study considered how workers' life strategies are influenced by social environments and beliefs. He found that workers had mixed feelings and ways of reinterpreting system change. Emotions associated with historical reflections affected current efforts to cope with the new system (Mrozowicki, 2011).

Using thematic analysis of experiences in working life and social environment compared against Archer's modes of reflexivity, Mrozowicki (2011) constructed a new set of modes. The main difference to Archer's typology, was the finding that whether reflexivity was community-focused or individual-focused affected respondents' understandings of their contexts and means to access resources. This was important in individuals' developing the ability to adjust to cultural change. Without access to resources, individual reflexivity alone was insufficient to enable people to act as they wished to, in response to societal change; they remained dependent on structures.

Mrozowicki's (2011) types emphasised agency-centred life strategies (integrating and constructing) and patterns of dependency and autonomy loss (getting by and embedding) which partially overlapped with other divisions within the typology. Individuals and families adapted or formed new ways to resist change regardless of their social position. The "development of hybridised ways of coping with social change" led Mrozowicki to conclude that the presence of values and resources alone was insufficient to "maintain biographical autonomy in the long run" (2011, p. 278). This finding challenged Archer's use of "contextual continuity" and "contextual discontinuity" and resources within social classes as keys to reflexive practice. 


\section{Caetano - personal reflexivity and possibilities}

Portuguese sociologists, Nico and Caetano (2015) developed Archer's understanding of reflexivity further, claiming the relationship between reflexivity, agency and social change could only be understood if the social conditions of possibilities are also understood. For reflexive action to occur, the social conditions must be right to allow reflexive deliberation to become creative action and produce change. This was a development on Archer's fractured reflexivity when in the activation of reflexive activities an individual decides they should do nothing about a concern. They may passively abandon projects, feel incapable of making a decision or actively pursue possibilities (Nico \& Caetano, 2015) but be unable to complete a project.

In an investigation of 20 individuals' personal reflexivity in their full biographies, Caetano (2015a) considered the practical dimensions of Bourdieu's habitus (social origins and socioeconomic resources) on internal conversation, using Lahire's external manifestations of reflexivity to examine reflexivity in external discourse (oral and written). According to Caetano, "personal reflexivity is understood as an internal mechanism of a person's mind, which is expressed through inner conversations" and mediated in external discourse (2017a, p. 35). Caetano looked at the choices people believed they could make, had made, and the decisions they acted on over time in light of political changes in Portuguese society. The assumption was that interaction with structures and institutions elicited a response in individuals and, in turn, the situation then determined an individual's response. She found the characteristics and purposes of reflexive thinking used were to think over a problem, contemplate the past, or to interpret a current situation (Caetano, 2015a).

Caetano's personal reflexivity typology took into account past contexts and events as well as present social conditions influencing reflexivity, based on life experiences (Caetano, 2015b, 2017a). Although asked about their individual experiences, in keeping with Archer's reflexivity and internal conversation concepts, all interviewees indicated they reflected on themselves and others while taking into account their wider social circumstances. The different ways or styles in which they exercised reflexivity led to Caetano's development of the reflexive modes of self-referential, pragmatic, functional, resistant and resilient reflexivity (Caetano, 2017a). The distinguishing characteristics of differences in socioeconomic background, sources of reflexive thinking, life concerns and projects, decision-making processes and dominant forms of exercise of reflexivity are presented in the typology. In a departure from Archer's claim of her reflexivity modes wider application, Caetano clearly identified her typology was specific to her study. 
In studies applying Archer's structure-agency dualism or adapting Archer's reflexivity and internal conversation methodology, none were found that examined direct challenges to students' values and beliefs. While there was consideration of habitus and contextual changes by some, only Matthews (2017) identified the discomfort felt by international students crossing cultural borders. Matthews also found the focus on student autonomy within UK higher education was at odds with international students prior learning experiences.

\subsubsection{External conversation}

In further development of Archer's reflexivity theory, following her empirical biographical study of Portuguese citizens (Caetano, 2015a, 2015b), Caetano (2017b) proposed a personal reflexivity perspective that draws more attention to and incorporates external conversations and discursive forms of reflexivity. These other forms of reflexivity are mediated by the interactions a person has with themselves and others in daily life. Rather than just looking at conversations as verification of ideas and adherence to the values of a social group, as Archer's communicative reflexivity uses, other interactions can be examined. Of particular interest are the conversations through one of the media Archer considered was breaking social groups apart - online social media formats. Social media and online discussion forums allow for an increase in communication of a different form and their role in reflexivity has yet to be explored.

Considering the exterior component of reflexivity alongside the interior brings more sociological depth. In social interactions, individuals define, negotiate and rework their goals and projects. These social contexts trigger and promote mental processes in wider conversations and for some people in writing. Caetano questioned 20 socially-diverse participants - 10 male and 10 females aged between 21 and 80 years (Caetano, 2015b) - about their writing habits, and their internal and external conversations of daily living. She found internal conversations activating reflexivity preceded most social exchanges. The focus of this reflexivity was on existing reality which was shared by others in their external dialogue (Caetano, 2017b). Certain social circumstances triggered reflexivity more than others; speakers could transpose their thoughts into speech or reflect on their own thinking during the interactions. In doing so, individuals observed themselves as a subject and their social conditions as objects (Caetano, 2017a).

Other people can be used as confidants, counsellors or listeners. They may be approached for advice, or to clarify thinking and sharing concerns. "Looking to family and friends with a reflexive purpose is a way to validate options and rationalisations" (Caetano, 2017b, p. 74). Often these ideas have been considered in internal thinking. 
"However, the exercise of reflexivity through external conversations does not occur homogeneously for all respondents and is crossed by axes of social differentiation" (Caetano, 2017b, p. 75). Caetano found external conversation as reflexive exercise varied across individuals depending on their educational, qualification and occupation level. Internal conversations prevailed in those with higher qualifications and salaries; externalisation of thoughts, ideas and evaluations was restricted to a few trusted people, including therapists. Those from less privileged socio-economic backgrounds valued and used conversations with a wide range of people as opportunities to reflect on issues, events, and relationships. Caetano attributed this to the importance of individuals' social networks for sharing their concerns and in seeking advice from others; the lack of material resources necessitated the use of emotional support systems (2017b). However, this social differentiation in use of opportunities for reflexive discussion based on economic and educational level may be particular to the context and focus of her study.

\subsubsection{Reflexive writing}

Writing as an additional means of reflexive processing allows the writer to take a distanced view of action. Caetano (2015a), drawing on Lahire's $(2008,2011)$ research on writing practice, saw the relationship between internal, external and written conversation was inextricably combined. "Writing generates reflexivity, stimulating new reflections, exploring existing ideas in more depth or approaching them in a new way. It could be said that writing reworks the reflexivity produced by internal and external conversations" (Caetano, 2015a, p. 68). Caetano (2017b) identified writing as an extension of internal dialogue, giving rise to new thinking and reflection or deepening pre-existing ideas. Autobiographical writing of lived situations allows the processing of thoughts and perceptions beyond past incidents and acts as a source of learning for future context. Formal writing can require ordering of thinking to support wider oral conversation. Organisational writing of lists, notes and schedules are identified as selfcontrol tools (Caetano, 2017b).

Written and external dialogue are normal actions and processes used in the social situations and interactions people engage with daily in contemporary society.

Both internal and external conversations are means of exercising reflexivity, ... are socially constituted, can occur simultaneously, are deeply connected to people's social backgrounds and action contexts, and express subjects' concerns and views of themselves, others and the world. This means that the external manifestation of reflexivity plays the same role as inner dialogues in the mediations of structure and agency. Structural constraints and enablements are 
received, filtered, and mobilised by subjects according to their concerns in the subjective definition and reflexive implementation of their personal projects through internal conversations, external dialogues, and writing practices. The activation of one or the other modality of reflexivity anchors in social differentiation processes and interaction dynamics that make individuals find specific answers in each way of exercising reflexivity (Caetano, 2017b, p. 84).

Like Archer's (2007) discussion of her reflexivity modes, Nico and Caetano (2015) also associated the intensity of internal reflexivity with higher education levels, qualifications and socioeconomic status. They claimed that individuals in these social contexts were not in the habit of sharing their concerns, as they can usually solved the dilemma in their own internal conversations. If they do discuss it with another person when they cannot solve a dilemma, they do not want to share their reflexive thoughts with familiars but will go to someone unknown (Nico \& Caetano, 2015).

If it is possible to understand how individuals' process knowledge, the social exchanges and structures they reference in their decision making, then it may be possible to recognise how much agency or freedom to act individuals feel they have to change their own immediate or wider circumstances. The process of their deliberations also indicates how open and flexible individuals are to new ideas, values and cultures encountered in globalised society. Caetano (2015a) argued that internal conversation is exercised discursively externally as a normal part of social existence to be able to collaborate with others. If it was not important in social interactions people would not do it.

\subsubsection{Emotions in reflexivity}

Two characteristics of internal conversation that emerged from two studies using Archer's reflexivity modes (Caetano, 2017b; Hung \& Appleton, 2016), but which are not developed by Archer, are the intensity of reflexivity and the emotion in reflexivity (Burkitt, 2016; Holmes, 2010, 2015). For something to be intense, it is a deeper, rigorous or more powerful encounter, implying some level of emotionality or responsiveness. Archer's definition of internal conversation is worded in a neutral manner which acknowledges emotion exists in the push-back effect against structures (Archer, 2007); however, this is not her focus.

Descriptions of the relationship between an individual's values and concerns and passive or active agents indicates a level of intensity. Active agents have a clear sense of self and place in the world, values they are prepared to stand by, goals and directions. To have a clear sense of self suggests an emotional investment in the meanings a person 
attributes to their identity and the social world, and in the selection of the self-presented to others (Burkitt, 2012, 2013). Meanwhile, passive agents experience disorientation and are unable to plan for themselves (Archer, 2007, 2012). Disorientation or confusion can be both intense and frustrating.

The intersection of the role of life concerns and socio-economic status in reflexivity is poorly understood. In a UK study of young people (18-24 years), in the care of the social welfare system, planning for their future outside of the state system, Hung and Appleton (2016) found those who were active participants with "rich and inventive" internal conversations had clear concerns and were fully engaged in projects consistent with their beliefs and values. Practical and "survival orientated" young people were self-reliant but with complex and flexible reflexive processes. They were ambivalent, aware that thinking made them anxious, or not fully perceptive of their situation. The researchers also found passive agents with "thin internal dialogue" found their internal conversations annoying or led to panic (Hung \& Appleton, 2016, p. 47). Caetano (2017a) found her Portuguese participants with resilient personal reflexivity or resistant personal reflexivity expressed motivational and emotional responses to their circumstances. Resilient individuals were still responding to the historical emotions developed from their experiences of warfare and these emotions inhibited their agency. Likewise, life contexts and concerns about socio-economic stability impacted on the reflexivity and action of resistant individuals. The emotional impact of reflexivity as a consequence of social circumstances is emerging as a new area of study in understanding the mechanism of the structure-agentagency relationship.

\subsubsection{Higher education and reflexivity}

The development of theory connecting Archer's structure-agency and reflexive processing modes and the morphogenesis of agency in higher education is relatively recent (see Case 2015, Kahn 2017 and Williams 2012). Higher education is both an institutional and social world, made up of visible and invisible structures, individuals and groups, at the interface of postmodern change. World-wide, students move between countries for educational opportunities bringing with them their cultures, experiences, values and concerns that may not match their new study context. In turn, local students bring their own sets of cultural and personal values and concerns. In universities, policies are developed in conjunction with government strategies and disseminated amongst staff and students (for example, the New Zealand Tertiary Education Strategy 2014-2019 (Ministry of Education, 2014)). Changes at policy level, such as the inclusion of graduate citizenship characteristics, are interpreted and applied by lecturers in the development 
of learning outcomes and changes to teaching practice to meet those requirements (Ahier et al., 2003; Arthur \& Bohlin, 2004). These macro-micro relationships affect every area of university study. Yet little is known about how macro-level changes affect the exercise of agency at the micro- or student level (Ashwin, 2008; Clegg, 2005; Williams, 2012). Nor, how free individuals are to decide on their own actions, how constrained they are by their own social contexts or the social setting in which they are participating (Ashwin, 2008). Indeed, Kahn (2017) argued that the current economic, or performative (Macfarlane, 2017), model dominating higher education "fails to satisfy the full range of students' aspirations, responsibilities and needs" (Kahn, 2017, p. 368). The impact of macro-level changes on individual agency can be understood through looking at agents and their responses and resistance to structures and changes.

Greater understanding of higher education students' contexts and their concerns is needed to recognise how they reflexively make sense of their studies and act on their new understandings. The possibilities for action and the changes they make at the microlevel (Clegg, 2005) are not just affected by the teaching and learning interaction (Ashwin, 2008) but by their personal, employment and study contexts. The assumption here is that people actively work to make sense of their world, rather than acquiesce to everything that is happening. "A strong understanding of agency is central to all forms of learning" as learning is shaped through interactions between social context and the individual, but the individual has a higher agential role (Williams, 2012, p. 32). However, while all experiences take place in the present, the present and agency in the present are enriched or shaped by the past (Emirbayer \& Mische, 1998). Only by understanding how people act in response to their learning can the extent of changes, the enablements and constraints of their social circumstances and the possibilities of new forms of action be understood (Case, 2013). This is of particular importance when considering diverse students in higher education.

The introduction of learning goals of citizenship and the implementation of courses focused on developing citizenship attributes make a number of assumptions about student learning contexts and cultures that may not recognise the diversity of circumstances and experiences which predate, allow or impact on the development of agency in citizenship. If indeed, humans are to have the capacity to "flourish" then they need the opportunity to exercise agency (Kahn, 2017). Rather than participation in study being just a performative task, the development of citizenship characteristics needs to be seen as a "morphogenesis of student agency" (Case, 2015, p. 140). Where agency is reflected in the way in which students "selectively recognise, locate, and implement" 
(Emirbayer \& Mische, 1998, p. 975) the attitudes, characteristics and behaviours they wish to incorporate into their identity.

Analytical dualism allows researchers to think through the relationships between structure and agency over time, and observe "the way in which personal identity and social identity come together" in learning (Case, 2015, p. 141). To act and shape society, students need to be able to "recognise ourselves as agents with the potential for collective agency" (Clegg, 2005, p. 160). However, as Bauman and Vecchi (2004) argued, the ability to express self-agency may be limited by the social setting, influences of past experiences, thinking about the future and engagement with the present. In this case, agency is contextualised both temporally and relationally (Burkitt, 2016; Emirbayer \& Mische, 1998).

If reflexivity on socialisation is accepted as a normal and on-going process, then the values and concerns that are a part of identity are also changeable.

Belonging and identity are not cut in rock, ... they are not secured by a lifelong guarantee, $\ldots$ they are eminently negotiable and revocable; and ... one's decisions, the steps one takes, the way one acts and the determination to stick by all that are crucial factors of both (Bauman \& Vecchi, 2004, p. 11).

The question of identity then arises when an individual is exposed to communities that are welded together by principles or ideas that are new, different or the same as their own. Higher education can have a physical or virtual social location, a place where social identities and personal identities are reconsidered in light of current social interactions, and other social contexts of family, work and extended community. All social locations bring their own sets of influences on the on-going shaping of the self and possibilities of the self for the future (Cross \& Markus, 1991; Markus \& Nurius, 1986; Rossiter, 2007). Therefore, the types of interactions which take place and the messages transferred are important in the construction of the self as a citizen.

A critical realist approach to student learning in higher education offers a means to critically consider how engagement in higher education affects social outcomes. Archer's mental activities of internal conversation and modes of reflexivity provide reference points for studies of reflexivity where values and concerns are challenged. However, the role of social, temporal and historical contexts in shaping ultimate concerns and priorities needs to be incorporated to understand agency within the structure-agent relationship. 
The contribution of Archer's reflexivity for understanding learning in higher education is that it involves "the whole person engaging with the world" (Williams, 2012, p. 319) allowing for a fuller understanding than cognitive reflective practice. Archer (2012) argued that reflexivity is the 'medium' used by agents to react to and make decisions in voluntary situations where there are different possibilities for moving through the world. It is how they make a place for themselves in their social contexts. While Archer focuses on future progression through social mobility and social class, progression from current social position and circumstances need not be restricted to social mobility. Instead, individuals exercise their personal capacity for reflexivity to deliberate about their self in relation to circumstances and their own contexts in order to plan future actions. They do not have to put plans into action immediately to demonstrate agency but must consider possibilities and decide on a course of action.

\subsection{Chapter summary}

In this chapter, I started by locating reflexivity's place in sociology and critical realism theory and its applications in post modernity. I then discussed how reflexivity operates within individuals' internalised deliberation and how individual realities are formed and change through socialisation. My intent was to demonstrate how reflexivity has been and can be used to explore individuals' realities, responsiveness to changes and challenges, and possibilities for change. I argued that in understanding individuals' reflexive processes we can expand our understanding of the complexities of the intersection of students' personal realities and learning in higher education, in a globalised and rapidly changing world where new ideas are introduced.

Social theory's increased consideration of reflexive thinking seeks to understand or make sense of these macro- and micro-interactions of social life and individual agency. Archer set out to address the lack of theorisation on the interplay between structures as external reality, how agency worked, and the influence of these interplays on an agent's decision making and actions. She proposed a morphogenetic approach as an explanatory framework for examining the effects of change on structures and agency. At an individual level, Archer argued a higher level of reflexivity is needed to challenge ideas, and to meet the challenges of morphogenesis.

Archer's use of the dualisms of structure and agency does allow for an unravelling of institutional and social webs of influences in personal reflexive deliberations. 
Understanding of individuals' values and concerns plays an important part in interpreting the dialogues of internal conversations and how people respond to structural and social change. Weaknesses in Archer's approach can be overcome by considering the wider social contexts, effects of socialisation and culture in responses to change. Looking at how reflexivity changes over time also offers opportunities to look at how personal history is consulted in reflexive thought.

I found no studies that examined students' reflexive thinking processes when their values and beliefs are directly challenged. Understanding how individuals' process new knowledge and the social contexts and exchanges they reference in doing so should provide an indication of how much agency or freedom individuals feel they have to act on their values and beliefs. Using the duality of the structure-agency relationship and Archer's reflexivity through internal conversation, as the medium for analysis of the effects of structures on agents and agents on structures, it should be possible to identify how university students respond when intentionally presented with values and ideas that differ from their own.

What has not been considered in current reflexivity studies and theory is how the processes of reflexivity works with individuals from diverse, bicultural and multicultural contexts. Intentionally presenting learners from different social backgrounds in a higher education context to rich, value-laden concepts (such as those considered in discussing citizenship) offers an opportunity to look at the reflexive processes of learners as they examine and critique presented ideas. Doing so opens reflexivity's processes to consider personal foundational values, the effects of past and current socialisation that influence personal priorities and concerns, and the influence of new understandings in triggering or endorsing personal projects. Understanding the reflexive deliberations and processes students use as they progress through a course of study provides an indication of the evolution of the invisible characteristics of graduate development (e.g. adaptability, resilience, tolerance, and openness) students want to demonstrate and society seeks to find in twenty-first century citizens. 


\section{Chapter 3. Methodology}

\subsection{Introduction}

In this chapter, I describe the methodology and methods used in this research. This research was designed as a qualitative longitudinal research study of the reflexive processes higher education students used to consider value-laden ideas encountered in their university courses. This formed the outer layer of a multi-layered embedded case study. The next case layer was a group of citizenship courses set within bicultural Aotearoa/New Zealand that intentionally introduced challenging concepts of citizenship attributes and values. I wanted to know how students processed these ideas and how much agency they felt they had to act on new understandings. The stories of the students as cohorts, and individual stories formed the units and sub-units of analysis.

A key assumption influencing my choice of study was that I believe higher education's diverse pool of learners do not leave their values, life concerns, social contexts and identities behind when they come to university. Through enrolling in a program of study, regardless of whether they study on- or off-campus, students engage with systems, cultures and people that are new or different from them. As a normal part of learning they consider the new ideas they encounter against their own contexts, personal reference points and prior knowledge. They make decisions about their present and future actions based on their considerations in reflexive thinking. In this chapter, I first discuss the ontological and epistemological viewpoints of Archer's (2003) critical realism and Berger and Luckmann's (1966) social construction. I then explain how I drew these two seemingly disparate theories together to use Archer's tools to determine students' reflexive and internal conversations through the separation of the dualism of structureagency.

Following this, I outline the study context, justify why I chose qualitative longitudinal research methodology and detail the research processes I used. After a discussion of the data collection methods, I describe why and how I used Lewis's (2007) framework analysis as a means of containing the various levels of analysis and charting of information. I did not initially set out to implement Archer's methodology, so I explain the modifications I made including the reasonings behind incorporating external 
conversations and written discourse in the data collection and analysis. In the final section, I introduce the citizenship courses and the contexts and commitments of the nine participants whose stories are analysed in-depth and presented in the findings and discussion chapters.

\subsection{Theoretical assumptions}

The goal of my research was to understand how university students deliberated in their internal and external reflexive processes, about their existing understandings and the new knowledge that challenged their thinking. I was interested in the social structures (family, culture, work, life) and influences (values, beliefs, concerns, priorities) they considered in responding to course knowledge, and their agency to act on their understandings. Qualitative educational researcher, Michael Crotty wrote:

Justification of our choice and particular use of methodology and methods is something that reaches into the assumptions about reality that we bring to our work. To ask about these assumptions is to ask about our theoretical perspective (1998, p. 2).

My theoretical perspective in this thesis merges Archer's critical realist ontology and epistemology with Berger and Luckmann's (1966) social construction epistemology; a prospect which Archer (2003) considered impossible.

As explained in Chapter 2, Archer wanted to explain how morphogenesis in reflexive thinking took place. Her focus was on the individual and the individual's changing view of reality in a period of rapid change. At an ontological level, Archer and Berger and Luckmann had similar ideas on the presence of external reality. That "as human beings, we necessarily live out our lives in all three orders of reality, natural, practical and social" with a "continuous sense of self" (Archer, 2000, p. 2). For Archer, physical objects existed and were described and accounted for using scientific process, likewise non-physical structures appeared real and are accepted by the individual. Berger and Luckmann agreed on the presence of external objects, or "“reality" as a quality appertaining to phenomena that we recognize as having a being independent of our own volition" (Berger \& Luckmann, 1966, p. 1). However, they attributed knowledge, "the certainty that phenomena are real and they possess specific characteristics" (Berger \& Luckmann, 1966 , p. 1) of phenomenon to social interaction and personal experience. Our understanding of the social world is made up of both what people tell us and what we experience. Thus, both acknowledged, entities existed as realities in our minds forming 
a part of our ordered reality, with the social world holding common understandings (Berger \& Pullberg, 1965). They differed in the importance placed on social interpretation of reality and the influence of the practical order and its structures on the self.

The differences and similarities in understandings of the self are important. A continuous sense of self is formed - by "our practical activity in the world" (Archer, 2000, p. 3). Archer believed the self was positioned between the power of structures and the influences of the social world. A "continuous sense of self " was ontologically stable; however, "our personal and social identities are epistemologically vulnerable" as people move through the world (Archer, 2000, p. 2). Personal reality was contained in internal thinking inaccessible to others. As an epistemological position Berger and Luckmann (1966) understood the practical world and the sense of self was continuously constructed and deconstructed in the social world through socialisation and reflected in the language used (discussed in Chapter 2). While Archer felt this gave too much power to the social world and agents (downward conflation), the social construction perspective was that the relationship of practical and social structures and agency was intertwined. It is this difference, and Archer's epistemological and methodological separation of the influences of structure-agency, that make her methods a powerful tool for unravelling how the sense of self and reality changes over time. It allowed me to open a window into the complex relationships between diverse higher education students' social and study worlds, their reality and the possibilities that exist for them to act.

In the remainder of the chapter I detail how I moulded Archer's methods and included variations from other researchers (e.g., Caetano) to gather, analyse and present the data.

\subsection{An embedded case study design}

In this research I used an embedded case study design. A case is defined "as a thing, as single entity, a unit around which there are boundaries" (Merriam, 1998, p. 27). Case study method suits investigations of real-life incidents or events while retaining a holistic understanding of the wider context and its characteristics and those of the event (Yin, 2009). In selecting the case, I was aware of Yin's (2009) caution of disagreement between researchers in the use of case study for descriptive exploratory research, particularly with issues of validity in comparing cases. However, I did not intend to make comparison between multiple cases, but I wanted to treat the case as an "integrated system with boundaries and working parts" (Stake, 1995, p. 2). This allowed me to observe the reflexive processes of learners within a specific higher education context. 
In complex scenarios such as this study, an embedded case study design can be used to encapsulate the various levels of investigation. Embedded case studies use either quantitative, qualitative or mixed methods and involve multiple levels, objects or units of analysis (Scholz \& Tietje, 2002; Yin, 2009). At the top level, this research is a case study about the internal and external deliberations of higher education students' considerations of knowledge in light of their personal realities. As such, it was an intrinsic study with my motivation for the study being interest in exploring the topic. At an epistemological level it is both exploratory and descriptive (Scholz \& Tietje, 2002) as I wanted to define what the reflexive processes were that individuals used, describe their reference points (structures, people and circumstances) and see how these aspects influenced individuals' agency.

To observe these characteristics, I selected a set of three citizenship courses in the Bachelor of Arts (BA) programme at one university over the period 2017-2019. This formed the bounds for the sub-case (Stake, 1994, 1995). The three courses focused on the development of students' public citizenship skills and capabilities. They were first incorporated into the BA degree in one Aotearoa/New Zealand university in 2017. All existing and new BA students were required to pass the courses to complete their undergraduate degree. The courses explored diverse and opposing cross-disciplinary concepts of personal citizenship identity on: individuals' sense of belonging and place within local and national spaces (at 100-level/first-year); the encounters and relationships between local, national and global communities (at 200-level/second-year); and what it means to be active citizens (at 300-level/third-year) (Kahu \& Gerrard, 2018). Together, these three courses form the sub-case of this research.

\subsubsection{The citizenship courses}

The citizenship courses are contextualised within Aotearoa/New Zealand, a bicultural country where citizens are ethnically identified as indigenous Māori, Pākehā/non- Māori of European descent, Pacific Peoples, Asian and other ethnic groups (Ministry of Social Development, 2016). The citizenship courses are provided in two modes: as an oncampus, face-to-face course and as a distance or online course. Distance students can study from their own home, workplace or on the go, with flexibility for their personal schedules. No campus attendance is required. Access to course learning materials and videos, activities (news, discussion forums and assessment platforms) and resources (library access and student support systems) are provided through the learning management system (LMS). Distance students taking the three citizenship courses formed the next level of my embedded case study. 
At 100-level, distance students were encouraged by the lecturer to talk to the people around them and to join in online discussion forums on the course content. Regular critical reflections of the course readings formed a part of course assessment. The 200level course required students to negotiate a small project (not implemented) with peers to consider a global issue and Aotearoa/New Zealand's obligations under international policy. Students were provided with access to software tools to complete group activities and assessment tasks. At 300-level, students canvased more widely to identify opportunities for participatory action, identified an action and worked with key stakeholders. These three cohort levels formed units of analysis, and the participants interviewed form the sub-units for analysis.

\subsubsection{Initial research questions}

Initially, I set out to look at the social influences on higher education students' deconstruction and reconstruction of their understanding of citizenship revealed in their internal deliberations, as they participated in a compulsory programme of study on citizenship.

Sub-questions I asked were:

- What knowledge, values and attitudes of citizenship are important to individuals' identities and sense of belonging?

- What social interactions contribute to participants' consolidation or change in knowledge, values, attitudes and beliefs over time?

- What are their internal and external reference points and how much agency do participants feel they have to change, or choose a course of action?

During data analysis, I became aware of the role of reflexive thinking in students' processing ideas of citizenship and refined my research question to focus on this (see Chapter 3.7.4. Revised research questions). Restricted access to the citizenship course materials and participant groups required a change in the study design and data analysis.

\subsection{Qualitative longitudinal research (QLR) methodology}

The goal of my research was to explore each participant's view of their reality and its influence on their learning context. As reality is socially located and each view is individualised (Berger \& Kellner, 1981; Cousin, 2009; Denzin \& Lincoln, 2000), to understand how reflexive knowledge was formed I needed to consider the social contexts, norms and interactions operating in students' lives over the period of their study (Crotty, 
1998). I needed to be able to recognise changes in individuals' understandings, their personal contexts and the influences these had on reflexive thoughts over time. The methodology used would inform how I gathered and reflected on the stories, experiences and voices of individuals over time.

I used Qualitative Longitudinal Research (QLR) to observe changes in individuals over time, to look for patterns of change and to understand the nature of time effects (J. Elliott, Holland, \& Thomson, 2012; Krathwohl, 2009; Saldana, 2003). Rather than looking for the causes and effect of change, I looked at what Saldana (2003) calls the "influences and affects" (p. 82) of change. Using QLR allowed me to look for contextual and commitment changes that occurred over the period of time students were engaged in study, using multiple data collection periods to produce rich descriptions of interactions at the individual or 'micro-' level (Bazeley, 2013; Saldana, 2003). The QLR approach also fits with my epistemological framework, underpinned by Archer's critical realism and Berger and Luckmann's social construction, as it draws on multiple methods to explore and analyse individuals' lived perceptions of reality.

A cohort-based QLR provides a basis to understand how individuals or groups may change over time. Specific contextual situations, events and personal characteristics can be examined to see if they contribute to the change (Krathwohl, 2009; Saldana, 2003). However, with the need for continuity between phases of data collection, Menard (2011) and Saldana (2003) advised the sample starting numbers needed to be higher than final numbers to reduce the effects of attrition. J. Elliott et al. (2012) also cautioned about the effects of participant conditioning from repeated interviewing or observation. However, I believed repeating elements within the interview designs would act as an effective way of understanding change in perspectives over time, and a more conversational approach to interviewing would counter conditional effects.

Other disadvantages of QLR which I also needed to consider were that longitudinal studies are resource intensive, and subject to participant drop-out and other changes from external forces (Krathwohl, 2009; Saldana, 2003). This is why there is a scarcity of 'real-time', small, low-funded qualitative longitudinal educational and social science studies reported in the literature that follow the same individuals or group over time (McLeod \& Thomson, 2009). Resource funding and reporting requirements can affect the alignment of interviewers with participants and restrict the type and frequency of the data collected. Participant drop-out can leave important gaps in data (Saldana, 2003). Therefore, the choices I made needed to be balanced with what could be achieved to maintain the credibility and integrity of the research and in reporting of findings. Tolich 
(2016) suggested that the actions researchers take to overcome the rough terrain of ethics, access, funding, recruitment and attrition should be recorded in notes of reflexive considerations, which I have done in this QLR project and included in reporting my findings.

\subsection{Manaakitanga - culturally and socially responsible research}

This research took place within Aotearoa/New Zealand, a country with a commitment to its bicultural, Māori-Pākehā context. Higher education in Aotearoa/New Zealand has commitments under the Te Tiriti o Waitangi/Treaty of Waitangi and the Tertiary Education Strategy (Ministry of Education, 2014) to move towards full biculturalism. I am a higher education teacher and researcher and Tauiwi (descendant of Pākehā colonialists). My family have two separate (paternal and maternal) generational waves of immigration, social and cultural change, and resettlement experiences. I am conscious of the impacts this has on identity and values, finding one's place and knowing where one belongs. On one side of my family I am a seventh generational Kiwi or Aotearoa/New Zealander and on the other I am a second-generation Kiwi. As a researcher, I am committed to manaakitanga/to show respect, generosity and care for others, in conducting culturally and socially responsible research. In developing my research recruitment and data collection strategy, I incorporated the values of the Ako Aronui Framework (Buissink, Diamond, Hallas, Swann, \& Sciascia, 2017) and the Ministry of Education's commitment to acknowledge Māori knowledge and perspectives within teaching and learning (Ministry of Education, 2014). Key values and principles which I hold and followed in this research are:

- Ensuring tika - my research is truthful and accurate in building knowledge and in acknowledging my participants' contribution to the common good (Austin-Wells, McDougall, \& Becker, 2006; Massey University, 2017b).

- I would build relationships/whanaungatanga with the participants through showing manaakitanga/respect for their cultures and values, and adapt my research processes to suit where necessary (Buissink et al., 2017).

- I would place people first to empower/whakamana them in upholding/whakamaranga and developing their understanding of their contribution to this research in affirming the expertise of their experience (Tracy, 2013).

In this section I have outlined why I used qualitative longitudinal research and planned for difficulties associated with this methodology. I also discussed my cultural, social and 
ethical responsibilities in conducting this research (ethics processes are detailed in Chapter 3.8). In the next section, I discuss the research processes and participant recruitment.

\subsection{Research processes and data collection}

The research was conducted over a four-year (2016-2019) period. Data were collected over several phases in 2017-2019 (Figure 3.1). Data collection, transcription and analysis processes set out in the research plan were modified and adapted as participants' circumstances, access to course artefacts and the format of the 300 -level course changed over time.

Initial analysis showed strong variability in the nature of students' processes of working through complex concepts of citizenship. As a consequence, the focus of the study moved from phenomenographic or interpretive research on students' development of understandings of citizenship to consider their reflexive deliberations on citizenship and its values.

\subsubsection{Research processes timeline}

The processes used in this QLR project are indicated in Figure 3.1. Issues with access delayed the first phase of recruitment until the second delivery of the 100-level citizenship course in 2017. Interviews and data collection began after the conclusion of the course delivery. 


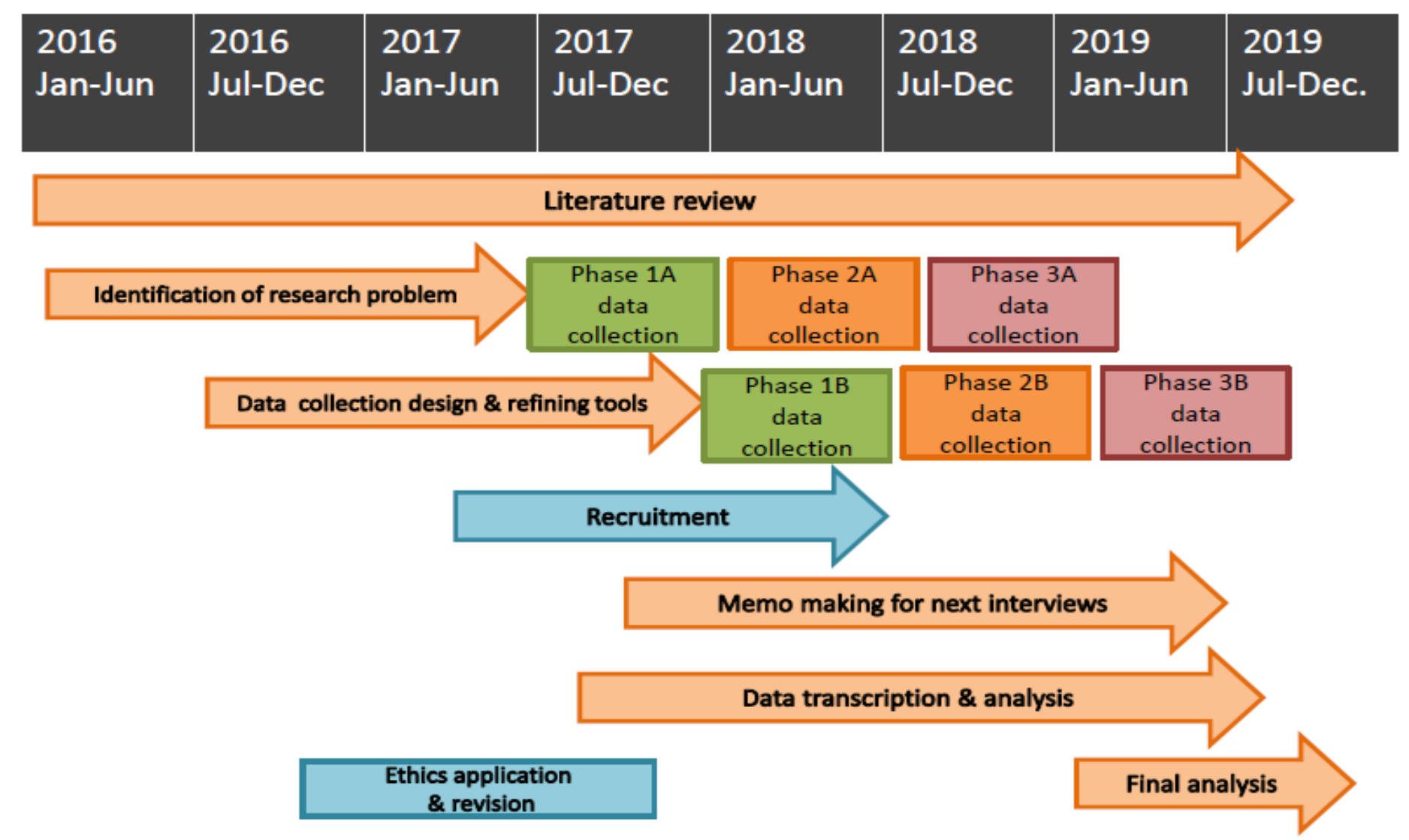

Figure 3.1 Timeline and overall design of the study 


\subsubsection{Participant recruitment}

Recruitment is the process of establishing dialogue between the researcher and people who meet the study participation specifications. In this study, the criteria for participant selection were students enrolled in a BA, Humanities and Social Sciences undergraduate degree, taking the citizenship courses by distance delivery and willing to commit to the research across all three courses during the period 2017-2019.

The number of participants was determined based on other studies and practicalities of the QLR research context. Adamson, Young, and Byles (2007) and Ribisl et al. (1996) note that studies rarely mention maximum and minimum numbers of participants required to meet academic rigor in longitudinal studies. My review of studies found articles, theses and dissertations of small-scale QLR studies on university students that report between 5 and 26 participant cases (Barnes, 2017; James, Taylor, \& Francis, 2014). Following discussions with my supervisors a goal of 30 initial participants was considered sufficient for this study. This would allow for variations in study patterns, participant drop-out, and management and analysis of the breadth of data that would be gathered.

I developed a recruitment and retention strategy in keeping with manaakitanga for initiating and following up on contacts made with participants. Seminal work on retention and attrition in QLR by Ribisl et al. (1996) showed inattention by researchers in followingup contacts with respondents plays a part in the loss of data. To build rapport and demonstrate my commitment and care for participants, I sent regular emails on the research process and progress and sought funding for giving koha/a gift for participants. Participants received supermarket, petrol or outdoor supplies vouchers. Attrition or the loss of participants can also be due to time constraints and changes in personal circumstances (McLeod \& Thomson, 2009; Saldana, 2003). Consequently, I took a flexible approach when scheduling interviews to fit into each student's work-life-study commitments. Interviews were conducted face-to-face using Zoom freeware. Interviews took place across various hours of the day and week while participants were at home, work, travelling and waiting on children.

\subsubsection{Recruitment issues and solutions}

Initial recruitment into the research did prove to be a problem. Participants were being asked to commit to this research for 18-24 months with a person unknown to them. I followed the recommendations of other QLR researchers to include information explaining the nature and importance of the research, requirements of participants, and 
ethical processes in place to protect their interests, confidentiality and privacy (Adamson et al., 2007; Ribisl et al., 1996; C. M. Sullivan, Rumptz, Campbell, Eby, \& Davidson II, 1996). I outlined how the research would proceed; the independence of the research from the BA programme, and how I would communicate with participants to build trust in the research and myself. I was also mindful of McGregor et al's (2010) advice that the research interaction should be an enjoyable and personalised social experience from the start to motivate participants to take part.

Multiple recruitment phases were needed to recruit the final total of 31 participants (Appendix A). A third-party administrator not connected to the course sent recruitment information by email to 317 distance students enrolled in the 100-level course prior to 2017 semester one commencement. Just two respondents sent in consent forms. A review of mail-out procedures and responses found the email was sent at the peak period of automatic emails from the university and LMS system. It may have been overlooked, diverted to junk mail, or blocked by the spam filters of the email provider (Toledano, Smith, Brook, Douglass, \& Elliott, 2015). To counter spam filters, the same administrator resent the email. After receiving no replies, the administrator provided me with LMS student email addresses and three weeks later I sent personalised emails. A further five respondents sent in consent forms. Thus, in total I had seven participants.

Adamson and Chojenta (2007) identified that specific research tactics are needed to recruit young people, mobile, vulnerable or hard to reach populations, like these distance learners. Toledano et al. (2015) used social media tools (Facebook and e-newsletters) to recruit, maintain contact with and keep research participants up to date with their study. Adamson and Chojenta (2007) also reported successful recruitment and retention approaches including creating a research identity, face or image that was presented to participants regularly. I decided the promotion and authentication of the project would need to be established through a person already known to the students and using social media. I created a Facebook page, with a logo for the research, included a photograph of myself, and incorporated these on all email correspondence.

Relying on the course lecturer's existing relationship with the students, I asked for the lecturer's assistance. They posted a notice about the research, its independence from the course, and details of the Facebook page and recruitment email on the course forum. This resulted in a further 17 students responding to bring the total to 24 at the end of phase 1A. At the commencement of semester one, 2018, I recruited a further 7 students from 151 enrolled students, bringing the final number of participants to 31 at Phase 2A. 


\subsubsection{Participant group}

The participant group was a diverse cohort of university students studying by online learning in an undergraduate Bachelor of Arts degree (Appendix B). In 2017, 40\% of 95,800 Aotearoa/New Zealand university students enrolled in either internal or distance delivery of the bachelor's degree were over 24 years of age (Ministry of Education, 2018). At the participating university in that same year, 13,796 (45\%) of the 30,883 students at the participating university were enrolled as distance students. Of all distance students, $54 \%$ were over the age of 24 years, $4 \%$ were Pacific Peoples and 10\% Māori. Sixty-three percent were female and 37\% male (Massey University, 2017c).

\subsection{Data collection}

Data was collected from student interviews, course assessments, online discussion forums, emails and researcher notes at the end of each of the citizenship courses in semester 1 2017, semester 1 and 22018 and semester 12019 semester. As outlined below, I used a combination of Reissman's narrative interviewing (1993) Josselson's relational interviewing methods (2013), and the participants' assistance to overcome issues with access to students' course artefacts.

\subsubsection{Semi-structured interviews}

I interviewed the participants face-to-face by video conference at the conclusion of each course. Using narrative questioning and relational interviewing methods, I gathered information on each individual's contexts, and their experiences of learning and their social processes (Appendix C). Josselson (2013) views the interview as a dance in establishing a relationship between the interviewee and interviewer. Both measure what can be said, how it is said, how much will be revealed through the engagement (Riessman, 1993). In interviewing I used pauses to allow interviewees time for reflection and framing of their answers. I was careful to use appropriate tones, gestures and expressions to make the communication as interactive as possible. Between sessions I reviewed my interview techniques and technical problems encountered to smooth the interview process for the next session.

Video conferencing by Zoom freeware allowed participants to pick the place and time of the interview. Zoom offered a simple no-cost tool (including video and audio recording) for face-to-face conversation across space and time zones (Zoom Video Communications, 2020). Participants could use it on smart phones, tablets or computers. While this was planned to give individuals some measure of control with the process and 
circumstances, to ensure they would be comfortable, this was not always the case. The interview space was occasionally shared or central to other activities in participants' homes, or a work break-out space. With participants' permission the video conference was recorded for transcription. Poor internet connections required perseverance or occasionally resulted in poor audio capture. Anticipating this, I jotted down notes as memory prompts for listening to the audio later.

I conducted interviews (45 to 80 minutes duration) with 31 participants at 100 -level to gather their perceptions of citizenship, to identify the influences on their deliberations, and how those deliberations occurred. I then followed these 100-level interviews with two more sets of interviews, after participants completed the 200-level and 300-level courses respectively. Due to course scheduling conflicts and changes in circumstances, participant numbers dropped at each stage from 31 at 100-level to 24 at 200-level. I ended up with nine full sets of interviews at the end of the data collection.

In the initial stages of the research, I wanted to find out how people made sense of citizenship and their own reality (Josselson, 2013; Ritchie et al., 2014). In one-to-one interviews I used 'grand tour' open-ended exploratory questions to create a conversational collaborative approach (Cousin, 2009; Josselson, 2013) in allowing students to talk about their experiences of learning. Follow-up questions requesting examples of 'experience' generated wider discussion of their involvement (Appendix C). Semi-structured interviewing techniques helped participants to focus as they described the constructed reality of their situation, exploring the meanings they had made and explaining them (Ritchie et al., 2014; Ritchie \& Spencer, 1994).

Semi-structured interviews allowed me "to develop more in-depth accounts of experiences and perceptions with individuals" (Cousin, 2009, p. 71). In treating the indepth interview as a "special kind of conversation" (Josselson, 2013, p. 180), I used open-ended guiding questions to investigate students' simple and complex experiences. I responded to the delicate balance between letting the participant discuss the ideas important to them and my seeking answers to particular research questions (Ritchie et al., 2014). Listening carefully, I would draw the discussion back to connect with the interview questions. For example, after a participant mentioned changes in their family circumstances, I would use bridging questions asking them how the changes in circumstances had affected their studies or thinking on citizenship.

I designed the interview as a conversation around a set of questions (Appendix $\mathrm{C}$ ) focused on my three main areas of research. The first focused on the participants' 
contexts, including their work-life-study circumstances, why they were studying, and the influences on their lives and values. In the second set, on the course content, I asked for their thoughts on their identity, citizenship and changes in understanding. The third set considered their citizenship actions and projections of future citizenship activity.

The same set of questions was asked of all participants; however, the sequence differed. I followed the natural flow of individual conversations, thoughts and directions using the questions to probe deeper and re-direct where necessary. Additional research questions recorded on memos made during transcription of previous interviews (discussed further in Chapter 3.5.3) were introduced into subsequent interviews. In the second or third interview, questions on Archer's definition of internal conversation and list of mental activities (2007) were incorporated (Appendix D) after initial data analysis revealed reflexive processing of ideas was an important part of the individual's learning.

The interviews, as a conversation between the participant and myself, were socially constructed at a point in time, "historically, politically and contextually bound" (Denzin \& Lincoln, 2013, p. 115). My role was not neutral, I played a part in the production of the narrative supplied by focusing, or placing boundaries around a particular topic or the question asked (Crotty, 1998; Denzin \& Lincoln, 2008; Josselson, 2013). I considered power an issue in these exchanges as I was both an outsider to participants' lives and course of study and a researcher. In several instances, interviewees sought to attribute me with the position of a course teacher which could have stopped them from speaking freely. I made adjustments in the interview style to introduce less formality and to reintroduce myself in each interview as an external observer of their engagement in their course (Denzin \& Lincoln, 2008).

\subsubsection{Interview transcription}

To understand an individual's construction of ideas I needed to foreground their words and explanations. To "enter the world of the participant and try to understand how it looks and feels from the participant's point of view" (Josselson, 2013, p. 80) required an empathetic ear during interviewing and responsiveness in preparation for further encounters. I made verbatim transcriptions of participant interviews as soon as practical, cross-checking the audio wording and noting word emphasis and emotions. Transcriptions included pauses, repetitions and idiomatic expressions (e.g., 'you know' and 'yeah'). These natural breaks in conversation (Cousin, 2009) placed emphasis on words or meanings, or participants used them to gather their thoughts. A benefit of checking the transcript with the recordings was that I could hear the nuances and 
emotions expressed in stories again and again and make notes of story segments requiring more clarity.

\subsubsection{Memos}

I prepared memos for subsequent interviews summarising a participant's context, interviewing conditions and my thoughts on ideas presented during the interview. Memos included the interviewee's verbatim quotes and my questions encouraging clarification or development of ideas for the next interview. Quotes from the memos were read back to participants with the qualifier "this is what you said in the last interview". This retrospective approach to interviewing and interim data analysis allowed each participant to clarify or elaborate on their comments. Participants were often surprised to hear what they had said, built on their comments, or referenced the dialogue as their thinking at a particular time. It also built a form of triangulation (discussed in Chapter 3.8). Using memos helped to build the researcher-participant relationship as they reflected empathetic responsiveness, manaakitanga/respect (Buissink et al., 2017), and a genuine interest in engaging with the participant in examining "the layers of experience" (Josselson, 2013, p. 84). Each participant's transcripts were later examined fully using framework data analysis methods discussed in Chapter 3.6.1.

\subsubsection{Reflective writings and discussion forum posts}

Documentary data from course activities, discussion forum postings and course assessments (critical reflections, short answers of key terms, essays and project reports) were requested from the LMS manager. Students' wrote their course assessments as responses to academic course requirements and they represent one form of voice directed to a tutor. Denzin and Lincoln (2000) identify voice as a multi-layered 'problem' representing the ideas of the writer, the responses of other voices within the data, and a portrayal of the self. Students' contributions to their peers in online discussion forums provided a secondary source of 'voice', audience, purpose and formality. To protect the privacy of other students, I was provided with only the postings of students who gave their consent rather than the whole conversation thread. As situated materials, they were developed in a particular genre and for academic purposes with a targeted audience in mind (Ritchie et al., 2014). However, they provided an indication of the student's thinking at particular points in time and I included them in the analysis.

Delays, incomplete information and difficulties in gathering 100-level online submissions from the LMS manager in semester one and two 2017, meant I used a different approach to gather this information in the 200 - and 300 -level courses. In subsequent interviews, I 
asked participants if they would provide me with an original (pre-marking) copy of the assessment materials they had submitted as coursework. This provided me with full sets of their written work. However, discussion forum posts (now described by students as more functional exchanges of group work or questions about course requirements) were not gathered at 200- and 300-level.

\subsection{Data analysis}

The component of analysis in this study is the cohort experience and the sub-unit the participant (Yin, 2009). I did a preliminary analysis at the cohort level and at each data collection stage of ideas or themes that related to the research sub-questions on personal values, concerns and priorities, and social interactions. I also identified the types of social interactions people used to contemplate ideas and made memos of discussion points. Thomson and Holland (2003) identify data analysis in QLR as an adaptive and evolving process as the researcher responds to their reflexive processing. While narrative analysis would allow retention of individual stories to be told as a whole (Riessman, 1993; Sparkes \& Smith, 2015), in my study the whole required all three stages of data collection to be completed. Meanwhile, I wanted to identify and explore ideas presented with participants in subsequent interviews.

Abundant advice exists in the literature on the handling of qualitative data (Lewis, 2007; Thomson \& Holland, 2003) and on thematic analysis (Guest, MacQueen, \& Namey, 2012), but I found little on managing data, on change over time in QLR, which incorporated recording researcher reflexivity at each stage. Research database software tools (e.g., NVivo, Q and MAXQDA) are recommended to store, code and retrieve information quickly (Bazeley, 2013; Guest et al., 2012; Ritchie et al., 2014). Initially, I tried to work within the machinations of NVivo but felt the processes created a barrier, distancing my reflexive thinking, and I sought an alternative.

To identify a participant's world view or experience I needed to use a context-based system analysis (Ritchie \& Spencer, 1994). A substantial volume of data existed from multiple interviews per person, participant generated writings, discussion forum posts, researcher-participant emails, and researcher notes and observations. A risk with QLR is it can reveal different types of change, but unless clearly planned these may be overlooked (Lewis, 2007). Each analysis approach privileges a particular understanding of change at the expense of other perspectives (Thomson \& Holland, 2003). Any interpretation I made would thus be provisional and temporal. I chose framework analysis method to manage the data for multilevel analysis - by theme across time without 
reducing the story over time, so each participant's voice, contexts and changes over time were retained.

\subsubsection{Framework analysis}

Framework analysis provides a structure for analysis based on a matrix system where data is ordered and summarised (Lewis, 2007, p 550). Developed by Ritchie and Spenser (1994), to look at the impact of UK health policy on patients' lives, Lewis's (2007) first stage of charting records the participant's personal backgrounds and a researcher summary for each participant interview. Like Thomson and Holland (2003), I found interviewees disclosed more background details and changes in contexts and thinking in each interview or piece of written work, so I adapted Lewis's levels of analysis worksheets to record those and to suit the goals of my research (Figure 3.2).

My cycles of analysis were:

1. Read and summarise transcripts, create memo for next interview - 100-level (T1), 200-level (T2), and 300-level (T3) interviews.

2. Read transcripts and written work to determine and compare themes and subthemes. Summarise themes and change over time.

3. Compare and summarise change between interviews and over study period.

4. Look for responses to Archer's mental activities and examples of internal and external conversation

5. Compare with Archer's modes of reflexivity (2007) and Caetano's (2017a) personal reflexivity modes. (This process and findings are discussed in Chapter $5)$.

Key themes from the research questions were identified. As I became familiar with the data, sub-themes from transcripts, written work and discussion forums were entered into charts (Figure 3.2 and Appendix E). Separate charts were drawn up to record themes and participants' accounts relevant to themes. Rows represent interviews or written documents and discussion forums at each time period of data collection. The columns represent themes (knowledge, attitudes, values, etc) and sub-themes (Figure 3.2, Cell 1-3). A sub-unit summary (the overall narrative and change in themes across time in personal accounts) was completed after the last interview and entered in Cell 4. This summary signposts back to detailed accounts from previous interviews, assessment and other data and includes significant quotes from participants. Thus, I was able to quickly recognise changes in context. 


\begin{tabular}{|c|c|c|c|c|c|c|c|c|}
\hline Participant\# & \multicolumn{8}{|c|}{ PARTICIPANT NAME } \\
\hline Subject Area & \multicolumn{8}{|c|}{ Citizenship } \\
\hline Theme & Knowledge & Attitudes & Values & $\begin{array}{c}\text { Social } \\
\text { Interactions }\end{array}$ & $\begin{array}{l}\text { Internal } \\
\text { reference }\end{array}$ & $\begin{array}{l}\text { External } \\
\text { reference }\end{array}$ & Agency & $\begin{array}{c}\text { Summation \& } \\
\text { memo }\end{array}$ \\
\hline Subtheme & & & & & & & & \\
\hline $\begin{array}{l}\text { 100-level (T1) } \\
\text { Interview }\end{array}$ & & & & & & & & $\begin{array}{c}\text { Summation \& } \\
\text { memo T1 }\end{array}$ \\
\hline \multicolumn{9}{|l|}{ T1 Other Text } \\
\hline $\begin{array}{l}\text { 200-level (T2) } \\
\text { Interview }\end{array}$ & & & & & & & & $\begin{array}{c}\text { Summation \& } \\
\text { memo T2 }\end{array}$ \\
\hline \multicolumn{9}{|l|}{ T2 Other Text } \\
\hline $\begin{array}{l}\text { 300-level (T3) } \\
\text { Interview }\end{array}$ & & & & & & & & $\begin{array}{c}\text { Summation \& } \\
\text { memo T3 }\end{array}$ \\
\hline \multicolumn{9}{|l|}{ T3 Other Text } \\
\hline \multicolumn{9}{|l|}{$\begin{array}{l}\text { Subthemes - } \\
\text { change over } \\
\text { time }\end{array}$} \\
\hline \multirow[t]{2}{*}{$\begin{array}{l}\text { Themes - } \\
\text { change over } \\
\text { time }\end{array}$} & & & & & & & & $\begin{array}{c}\text { Summary of } \\
\text { change over time }\end{array}$ \\
\hline & \multicolumn{3}{|c|}{ Cell 1} & Cell 2 & \multicolumn{2}{|c|}{ Cell 3} & Cell 4 & Cell 5 \\
\hline
\end{tabular}

Figure 3.2 Framework data analysis charts for this study (based on Lewis, 2007) 


\subsubsection{Types of narrative change}

I looked for four types of change - narrative change, participant reinterpretation, researcher interpretation and no change following Lewis's (2007) analysis guidelines. Narrative change is where an individual's stories developed over time. Stories are not always told in a linear manner (Lewis, 2007); rather people move forward, back and across events, ideas and time (Vogl, Zartler, Schmidt, \& Rieder, 2018). People may not reveal themselves and ideas to the researcher in the initial stages of research as research participation is not normal for individuals (Thomson \& Holland, 2003). During the second interview of my study, one participant explained how fragile she had been in the first interview due to a recent relationship break-up. This is an example of the interview as a co-construction between interviewee and interviewer (Josselson, 2013). The participant had screened what she presented. While I noted her distress in my research notes, I did not ask about her situation in the interview. Thomson and Holland (2003) observed that the interview can have an impact on a participant's thinking as they respond to and process questions. It may have been the interview itself which stirred up an emotional response.

Participants reinterpreted their own stories as events once meaningful become more or less so with time. Their understanding of experiences and the importance of, or meaning given to, interactions changed over the study. For example, in the second and third interview, one participant discussed how she now recognised her discussion forums posting in the first course as racist and had not recognised this earlier. Lewis (2007) calls this "implicit reinterpretation" (p. 549) where the reasons why stories are reframed may not be immediately obvious to either the teller or listener. In interview two, the weight of a family incident influenced and limited one participant's responses to questions. It was only in interview three that I understood the impact on interview two's distanced replies when the participant returned to their previous demeanour and chatty style. This change in my interpretation reflected my development of, and confidence in, my research skills to reconsider the earlier interview (Thomson \& Holland, 2003). I recorded this in my notes and report it in my retelling (in Chapter 4.1).

As people do not experience situations in the same way as others, I looked for what remained the same "or consistent over time" (Saldana, 2003, p. 114). The absence of change, or change in many subtle forms, is just as revealing as other forms of change (Lewis, 2007), an aspect disregarded by Vogl et al. (2018). This was important in my study as it recognised that mature students, and those who had lived or were living in other countries, had well-established concepts of citizenship and no obvious change in 
concepts took place. What did change were smaller details in understanding of themselves or interactions with others. This is where the intricate nature of the framework approach allowed me to weave back and forth across participants' stories, themes, and time to observe these subtle changes.

\subsubsection{Themes}

I used the first two participants' interview transcripts to deductively identify themes that related to the research questions and then applied these themes to my analysis of the other interview transcripts, discussion forum posts and assessment work. As I progressed through my analysis I refined and adapted themes where necessary (Table 3.1). For example, I added place/locality as a 'value' after two participants compared Aotearoa/New Zealand attributes (of openness to other cultures, encouraging voting in political elections, and care for the environment) with those in their country of residence. Place was also added under concerns and priorities after issues at global, national, regional and local levels were raised, for example moving Aotearoa/New Zealanders out of poverty before allocating aid overseas. These themes identified information directly answering my research questions and formed a common framework for comparison of each participant's interviews and writings to reveal changes over time. Themes provided a structure for textual evidence in story analysis. They are incorporated in the findings in my next chapter.

Table 3.1 Analytical themes generated from participant data

\begin{tabular}{|l|l|l|}
\hline Personal description: & Influences: & Values: \\
Identity roles & People & Environment \\
Identity as place & History & Family \\
Group identity & Current context & Culture \\
Work- study status & Religion-faith & Religion \\
Age & Culture & Upbringing \\
Gender & & \\
Change in identity descriptors & & Concerns and Priorities: \\
New Knowledge/New thinking: & Processing ideas: & Family \\
Trigger ideas from course & Talking to self & Economic \\
Triggers from social interaction & Talking to family & Study \\
Trigger from research & Peer Discussion & Work \\
Future self & Other Discussion & Relationships \\
& Writing & Place/locality \\
\hline
\end{tabular}

This section outlined data analysis approaches used and other aspects I considered by which to gain a coherent understanding of a participant's perspectives of citizenship. Interim findings from data analysis in 100-level interviews drew me to look at 
interviewees' inner and external processes and reference points that they used to consider the value-laden ideas of citizenship.

\subsubsection{Revised research questions}

As a consequence of my initial analysis, I revised my main research question from the broader scope of diverse higher education students' experiences and construction of ideas of citizenship to focus on the reflexive processing of ideas.

The research question became:

How do diverse higher education students reflexively process and act on value-laden knowledge presented in their studies?

To answer this question, my sub-questions were:

- What knowledge, values and concerns are important in individuals' reflexive processing during their citizenship courses?

- What social interactions are important in considering new ideas from the citizenship courses?

- How do these internal and external conversations work?

- How much agency do individuals feel they have to act out their reflexive processing?

Having laid out in this section the research objectives, the methods used to recruit individuals, gather and analyse data and shift in direction, in the next section I present two phases of the study.

\subsection{A note on the codes used to identify participant quotes}

In the next three chapters participant quotes are used to provide representation of an idea (Riessman, 1993). The quote is followed by the quote source - coded and enclosed by brackets - using the codes described in Table 3.2 The codes differentiate between course levels (e.g., Ch100, Ch200, Ch300), the more spontaneous comments from the interview (participant initials e.g., Ch, $\mathrm{Kr}$, or name), and more considered thinking posted on the discussion forums (DF) and in emails (EM) sent to me. The more purposeful and considered written assignment responses are indicated by WW. When presenting and discussing the 100-level and 200-level cohorts only initials are given for participants. At 300-level the pseudonyms Jem, Elsie, Nan, Kane, Karen, Nell, Deedee, Sage and Shazz are used rather than initials. 
In repeating participant quotes, the participant's wording is italicised throughout my thesis to differentiate these from excerpts from the literature. Natural breaks in conversation pauses, repetitions and idiomatic expressions (such as 'you know' and 'like') have been removed to provide a clearer presentation. As semiotics and structural analysis (Bazeley, 2013) are not a part of this research, participant tones, gestures and emotions have not been included.

Table 3.2 Identification codes used with quotes

\begin{tabular}{|c|c|}
\hline Code & Description \\
\hline $\begin{array}{l}\text { Te100 } \\
\text { Te100DF } \\
\text { Te100WW } \\
\text { Te100EM }\end{array}$ & $\begin{array}{l}\text { Participant Ch* 100-level Interview } \\
\text { Participant Ch 100-level Discussion Forum } \\
\text { Participant Ch 100-level Written Work } \\
\text { Participant Ch 100-level Email }\end{array}$ \\
\hline $\begin{array}{l}\text { Te200 } \\
\text { Te200WW } \\
\text { Te200EM }\end{array}$ & $\begin{array}{l}\text { Participant Ch 200-level Interview } \\
\text { Participant Ch 200-level Written Work } \\
\text { Participant Ch 200-level Email }\end{array}$ \\
\hline $\begin{array}{l}\text { Te300 } \\
\text { Te300WW } \\
\text { Te300EM }\end{array}$ & $\begin{array}{l}\text { Participant Ch 300-level Interview } \\
\text { Participant Ch 300-level Written Work } \\
\text { Participant Ch 300-level Email }\end{array}$ \\
\hline $\begin{array}{r}\text { * In the em } \\
\text { Elsie } \\
\text { are }\end{array}$ & $\begin{array}{l}\text { bedded sub-unit, the participant pseudonyms Jem, } \\
\text { used. }\end{array}$ \\
\hline
\end{tabular}

\subsection{Ethics}

As indicated in Chapter 3.4, I am committed to manaakitanga in conducting culturally and socially responsible research. I read widely, researched and consulted with Māori researchers on my approaches, actions and interactions with participants in recruitment and retention strategies, interview conduct, and in giving koha/gifts (e.g., vouchers) to acknowledge my participants' contributions. 
Following Victoria University of Wellington (VUW) Human Ethics Policy (Victoria University of Wellington, 2016) and using Cousins (2009), Denzin and Lincoln (2013), and Tolich (2016), I developed my ethical framework. Ethics consent was approved by Victoria University of Wellington (VUW) Human Ethics Committee (Appendix F). An amendment to the consent was subsequently approved to provide vouchers as koha/gift for participants to acknowledge their contribution to my research.

Consent was sought from the case study university to approach students enrolled in the citizenship courses. In initial discussions with university managers, concerns were raised regarding time pressures on teaching staff and the on-campus students in the new courses. This led to a decision not to include staff and on-campus students in the research. To maintain the independence of the research and my position as researcher, I decided all communication with the students would be made in conjunction with my role as a $\mathrm{PhD}$ research student at Victoria University of Wellington, which was not the research site. Other conditions were addressed after managers raised reservations about an external university having access to proprietary knowledge about the new courses. This was mitigated by my supervisors signing a confidentiality agreement. Subsequent negotiations resulted in my agreement not to access teaching and learning materials from the learning management system (LMS) and to restrict my observations to distance learning students. Agreement was also reached on the processes for gathering student artefacts submitted to the LMS.

\subsubsection{Trustworthiness}

Trustworthiness and integrity are important in my research. As discussed in Chapter 3.4 measures to conduct socially and culturally appropriate research were taken. To protect the identities and privacy of my participants and their families, I have used pseudonyms and generalised location and employment information in telling their stories. I have used an independent reviewer to check for potential lapses in the de-identified data. To provide assurance that my qualitative longitudinal research is credible, confirmable, dependable and to build trust in the research outcomes (Guba \& Lincoln, 1998), I have provided transparency in my management of research and analysis processes and kept notes of "critical self-reflection or reflexivity" over the research (Bazeley, 2013, p. 407).

In the study I focused on participants' changes in understandings and reflexive thinking over time. Multiple in-depth interviews provided opportunities for participants to tell and retell their experiences, the meanings they held and their meaning-making processes. I followed Yin's (2009) three principles of data collection that support the validity and reliability of evidence. I used multiple sources of data, organised and contained the 
different forms of data and my summaries using framework analysis tools and methods; I maintained the chain of evidence outlined in this chapter. Email exchanges, written course assessments and discussion fora contributions provided a form of triangulation of data sources to allow me to compare my interpretation of what I was seeing across the data (Bazeley, 2013; Ritchie et al., 2014). By comparing this data in analysis for confirmatory, contradictory or absence of statements (Lewis, 2007), I believe I have represented participants' views with accuracy, although, my interpretation is always conditional and open to other explanations.

Theoretical triangulation, the process of looking at data from different theoretical positions (Ritchie et al., 2014), was a part of my researcher reflexivity, data analysis and discussion of findings. This research sits at the convergence of sociological and higher education theory. Social constructionist theory (for example, Berger \& Luckmann, 1966; Elder-Vass, 2012; Gergen, 2011), interpretive social structuration theory (Giddens \& Cassell, 1993; Giddens \& Sutton, 2014) and critical realism structure-agency and reflexivity theory (including Archer, 2007; Archer, 2010a, 2010b; Caetano, 2014, 2015a, 2017b) provided the lenses through which participants' social contexts, reflexivity and agency were considered. Higher education theory of Biesta and Simons (2009), Kahu (2013), Kaufman (2014) and others provided further reference points.

I did not use member checking of interview transcripts and interpretation as a means to assure confirmability as this would also raise ethical issues of my obligation to change data if requested (Bazeley, 2013). Instead, I used multiple sources of data and asked follow-up questions during interviews and in emails, to confirm my interpretation of participants' thoughts and understandings with them. Lewis (2007) identified that changes in narratives or storytelling over time is normal as adjustments in participants' circumstances and reflections occur. I drew on relational interviewing techniques (Josselson, 2013) for detailing individuals' stories and clarification of my understandings, checking both within and between interviews. Follow-up questions for clarification or participant responses were 'reflected back' in interviews by me to check understandings (Josselson, 2013). Memos for subsequent interviews included my reflections of the interview and context, interviewee quotes and questions framed to follow-up on earlier responses (see Appendix $G$ for an example).

Framework analysis (discussed in Chapter 3.7.1) provided me with a strong foundation for ensuring consistency in data analysis (Kiernan \& Hill, 2018; Lewis, 2007; J. Smith \& Firth, 2011). These actions should reassure other researchers as they make judgments about the application of this study to other contexts. 


\subsection{Chapter summary}

In this chapter, I have presented the epistemological assumptions behind my study, the use of embedded case design and my qualitative longitudinal research approach. I have acknowledged my commitment and adherence to recognising Te Tiriti $\mathrm{O}$ Waitangi and Aotearoa/New Zealand's bicultural context in my research methods. I have described the recruitment and data collection processes, framework analysis and how I used it to analyse the collected data. My justification for and actions in conducting ethical, social and culturally reasonable research were explained. The steps I took to meet ethical requirements, recruit and retain participants and document the research process to establish a chain of evidence have been discussed. In the next two chapters, I present the findings of my research. 


\section{Chapter 4. Findings from framework analysis}

Diverse higher education students' reflexive considerations of their values and concerns and the enablements and constraints of their circumstances, provide an indication of their agency to act on new understandings. In this section, I present my findings from analysing distance students' stories, writings and discussion forum posts. First, I give an overview of the participant group and how the contexts and commitments affected their study programme. I then provide an overview of all the participants' attitudes towards the citizenship core courses. I evidence this with quotes from participants. Identification codes for quotes include initials of pseudonyms, interview number and type of data are provided in Table 3.2.

This is followed by descriptions of the 31 participants' learning experiences in a first-year course, intentionally designed to challenge their thinking about their identity and what it means to belong. I then describe the participation of the 24 students who continued on to study global citizenship in the 200-level course, and how changes in their study patterns and personal circumstances affected their subsequent participation in this project. In the third part, I present the experiences of nine participants at the 300 -level within the data collection period.

\subsection{Participants' contexts and commitments}

Thirty-one distance study BA students volunteered and consented for their stories and course contributions to be gathered in this study. Students studying by distance have complex work-study-life contexts (Appendix B). Participants ranged in age from late teenage years to those in their 50s. Six participants (19\%) were male, which is lower than that of the overall student population (37\%) at the participating university in 2017 (see Chapter 3.5.4). At the start of the research, 21 of the 31 participants identified as being employed full-time and studying part-time (13) or studying full-time (8) (Table 4.1). Six were studying part-time while in part-time employment and four were studying parttime. Occupations described in identity roles included: student, mother, unemployed, public service worker, factory worker, and teacher. The number of participants in this study who described their ethnicity as Māori (23\%) or Pacific Peoples (6\%) (Table 4.1 and Appendix B) indicated this cohort had higher representation of these ethnic groups 
than the overall student population who selected these categories on enrolment at the participating university.

Table 4.1 Participant age, work-study status and ethnicity

\begin{tabular}{|lc|}
\hline Participant Age Range & Percentage \\
\hline$<20$ years & $2 \%$ \\
$20-29$ years & $32 \%$ \\
$30-39$ years & $29 \%$ \\
$40-49$ years & $20 \%$ \\
$>50$ years & $16 \%$ \\
\hline Work-Study Status & Percentage \\
\hline Full-time work and Part-time study & $39 \%$ \\
Part-time work and Full-time study & $26 \%$ \\
Full-time study & $9 \%$ \\
Part-time study & $26 \%$ \\
\hline Ethnicity & Percentage \\
\hline Māori & $23 \%$ \\
Pacific Peoples & $6 \%$ \\
European immigrant & $10 \%$ \\
New Zealander/Pākehā & $61 \%$ \\
\hline
\end{tabular}

Major changes in individuals' circumstances affected their study experiences over the research, including changes in employment, locality, parenting, relationships, and completion of study. During data collection, seven participants' employment situations and locations changed. Another three experienced relationship breakdowns and three gave birth. Six participants ceased study due to health reasons or major lifestyle changes, including relocating to other cities or countries for employment. The complexity of these work-life-study situations is typical of distance learners in Aotearoa/New Zealand (Brown, Hughes, Keppell, Hard, \& Smith, 2015; Guiney, 2014; Kahu et al., 2014). Such situations affect study commitment, resources and the time available for knowledge development, completing tasks and progression through their studies.

Due to the staggered nature of full-time and part-time study, course prerequisites and variations in participants' personal circumstances, 31 participants completed the 100level course, while 24 completed the 200 -level and only seven participants completed all three courses in the 2017-2019 period. I also interviewed two others at the 300-level who wished to stay on in the research project, despite not yet having enrolled in the 300-level course. This brought the total number of 300-level participants to nine, and I describe the findings from those nine participants in much more detail in the next chapter. 
Meanwhile, in the next section, I present an analysis of all 31 participants' views of the citizenship courses as a required part of their studies.

\subsection{Citizenship as an external reflexive conversation}

The goal of my research was to understand how university students used reflexive processes in their internal and external deliberations of understandings of citizenship. Active participation in the citizenship courses encouraged a discursive component of reflexivity through asking learners to discuss core concepts with people in the wider community. To this end, I considered external discussions of citizenship as a reflexive conversation. External social conversations can reflect internal deliberations in oral, visual and written formats (Caetano, 2017b; Chalari, 2007). This exchange of ideas required a level of symbolic mastery of language and for students to translate their understandings of curriculum ideas into their everyday lives or course activities. This opened up the opportunity for students to consider ideas and values of citizenship beyond Aotearoa/New Zealand society and higher education contexts. I wanted to know the role played by knowledge, values and concerns in how individuals' reflexively processed ideas, and the role that others played in their consideration of new ideas.

\subsection{Attitudes towards the courses}

The three citizenship courses are core to the BA curriculum and students' development as citizens within the university environment. The goal was to build student capabilities as local and global citizens and as graduates for global employment (Massey University, 2017a). To complete their degree, students must pass all three core courses. The core courses were introduced mid-way through some students' study programme, without a transitional phase, and required all current and future students to complete the courses. This may have impacted on participants' study choices, engagement with learning and participation in course activities, all agentic activities. From my analysis of the interview data from 31 participants' reactions to the inclusion of the core courses into the BA, I identified three main themes: opposition, resistance and acceptance.

\subsubsection{Opposition}

The theme of opposition captured the reactions of those who expressed emotions of dislike or 'hate', annoyance and 'not-in-this-degree' attitudes. Opposition encompassed those who were resolutely against any change to their study programme and included those who resented the citizenship courses being imposed on them. Having to take a 
prescribed set of courses was viewed as a constraint on their progress in completing their degree and on the quality of the qualification. Six mature students, with clearly articulated identities, argued that the courses addressed concepts of which they already had a firm understanding. Along with two younger students who were mid-way through their degrees, these mature students resented having to limit their specialisation electives to add the courses into their study programme. They experienced a lack of agency through this in their lack of choice. While some acknowledged that opposition was their starting point and remained their position, they saw some value in everyone exploring citizenship ideas. Indeed, in the interview one mature student acknowledged the courses' usefulness in "creating a sense of belonging in a Bachelor of social sciences" and "a collective base to work from in the unknown of university" (Ka100). Initially annoyed, another mature student found "this course was dealing with some themes I am already fairly familiar with, but it was good for me" (Ja100). It prompted another student with the opportunity to discuss with their adoptive parents the impact their lack of knowledge of whakapapa/ancestry had on their identity.

\subsubsection{Resistance}

Nine other students also expressed their concern that they did not want to limit their course choices or to be told what to do. However, as they accepted the courses were now a part of their degree, I used the theme of resistance to represent their views. They sat on-the-fence, balanced in weighing up the restrictions placed on their choices without being able to clearly see the benefits of doing the citizenship courses. This group included both new students and those midway through their degrees. One young, firstin-the-family to university, student pointed out that having left compulsory education where they had restricted choice on subjects, they believed in and expected freedom of choice at university. The imposition of a mandatory course felt like an impingement on their rights.

Despite their lack of support for the courses being compulsory and restrictions on their choices, this group did not voice the resentment of the opposition group. Notwithstanding the restricted freedom, the course content was still considered "important for everyone, for society, for life skills, putting into words the things that connect us with society. It's applicable to students but you as a person in everyday life, society and culture" (Je100). Two European immigrants felt their fellow learners should have already developed understanding of their citizenship history through their schooling, as they had in their birth countries. However, they personally appreciated engaging with Aotearoa/New Zealand culture through the course. 


\subsubsection{Acceptance}

The theme that encapsulated the stance of just over half (16) of the students is acceptance; they acknowledged and accepted the citizenship core as a part of their studies. Overall, these students found the experience helpful. First-year students, those identifying as Māori or those familiar with core curriculum components, saw the nonelectives as enablements in preparation for life or a stepping-stone, preparing them for their degree. Another first-in-the-family at university student described the 100-level course as achievable and interactive, which gave them confidence for further study. The remaining students were enthusiastic about the course - summed up in the interviews of two respondents as "fabulous, brilliant, people are being forced to learn about who they are, about our country's identity" (Tr100) and "fundamental to anyone's higher education in New Zealand. They just really set the foundation for things that are important and not only for writing and inquiry and critical thinking but just the way they enter into other studies" (Da100). They didn't feel constrained or restricted in their choices but viewed the courses in a positive manner.

Overall, while half the 31 participants were enthusiastic about the course, some participants were strongly opposed to the substance of the course and resentful of having to participate. At this point I wondered if this might affect their consideration of citizenship knowledge across the three courses and engagement with course activities. However, the change in direction between study levels (from personal citizenship at 100-level to global citizenship at 200-level) added sufficient novel interest to maintain their attention. By the completion of all three courses, most of the nine 300-level students who had originally been opposed or resistant to the courses $(n=6)$ identified what they saw as personal or social benefits of having to engage and reflect on multiple perspectives of the values and concerns of citizenship. Three mature students remained resistant, still focused on the restrictions imposed on their study choices. However, they had chosen to volunteer in this research as an opportunity to express their thoughts.

Understanding the participants' attitudes towards the courses needed to be taken into account in looking at change in reflexive thinking over time.

\subsection{Reference points for processing citizenship knowledge}

Students' reference points and the processes they used to understand curriculum ideas in each course are presented in this section. The first-year/100-level course (31 students interviewed) explored personal identity and ideas of belonging, introducing students to a 
number of conceptual threads woven through all three courses. The 200 -level/secondyear course (24 students interviewed) moved from emphasising personal citizenship to national and global citizenship. Students worked in groups to consider global issues through universal principles presented in United Nations documents to which Aotearoa/New Zealand is a signatory. The final/300-level course (seven students interviewed) examined what it means to actively participate as a citizen. Students selected an issue for investigation, planned, researched and implemented an action; reporting on the action in their assessment. I interviewed a further two students, who would have completed the 300-level course at the time had circumstances not prevented them from doing so. These two students, and the seven who did complete, form the basis for the majority of the in-depth findings in the next chapter.

\subsubsection{Personal identity and citizenship - 100-level}

The first-year citizenship course starts with concepts of identity, belonging, participation, context, and citizenship. The course then has four modules: Faces - Māori as tangata whenua (indigenous), and the current make-up of New Zealand; Voices - how people express their identities through politics, protest, and art; Places - how identity is shaped by the places where we encounter others (university, home, and digital); and Stories - a critical look at three narratives that shape our national identity (egalitarianism, clean and green, and Anzac - a defining event in national identity) (Kahu \& Gerrard, 2018, p. 72) .

The courses were designed with threads that form linkages across all three courses. Students were expected to read the course text and other academic and popular readings. They submitted small (300-word) critical reflections weekly on a course idea, or thread, as well as essays. The 100-level course lecturer encouraged participants to talk with other people outside the university, in their workplace and social activities, and to join and contribute to online forums. I asked the participants what their personal values and priorities were, what insights they had gathered, who they discussed citizenship ideas with and what particular conversations they engaged in. Major themes from participant interviews then identified the people they consulted, and the processes they used to clarify their understandings of the course threads.

Time spent in internalised deep thinking about course ideas, while doing routine or mundane tasks, or in preparation for writing critical reflections was identified as normal for one third $(n=10)$ of the participants. In what Archer (2007) calls internal deliberation, they would mull over ideas which challenged their thinking. They felt deep rumination "let 
you move beyond the text" to really think (Ke100). Examination of the written critical reflections revealed half of these students related the readings to their own lives and experiences. For example, how being Māori, Polynesian, or a state beneficiary had affected their own lives or their parents' lives. Another three related ideas directly to their work in social services, in particular whether the general public they encountered reflected a truly bicultural or multicultural country. Connections made with lived experience helped understandings of how identity and belonging were formed.

Talking ideas over with others was important for half the participants to gather new ideas, clarify their own thoughts or test out their own thinking. These social discussions helped them come to a decision in their own thinking. The main people they consulted were close family (parents, siblings, children and partners), followed by friends (trusted friends and networks of friends) and workmates. As one student explained, talking with others "expands what I have heard and [gives me] their thinking" (Jc100). Others wove their conversation on citizenship topics into their everyday lives, talking with their children's friends and other parents at family and community activities.

Talking over the course ideas with others also allowed some participants to reconnect with their family history. While reading about Aotearoa/New Zealand protest movements, a male student, recognised a family name in the text. This led him to talk with a family member, which led to a snowball effect in contacting other extended family members to discover more information. In making the connections with this historical event and family connections to the ANZAC (Australian and New Zealand Army Corps), the student recognised how his family history and values had been passed to his own generation. Values he recognised in himself.

Some used their participation in local community activities to target specific demographic groups - senior citizens, young solo parents or first-time voters - to discuss specific topics. Authoritative people - authors, lecturers and respected family members - were approached for their thoughts. Two students emailed the authors of course readings; another two talked to educated and socially or politically active siblings. Five students working in the public sector gathered colleagues' opinions or tested their own thoughts with colleagues. However, two students refrained from talking to fellow contract and factory workers fearing knowledge of their studies might jeopardise their employment.

Social media platforms provided the discussion mechanism for socially isolated students (living in rural areas, living overseas, those housebound due to health, or where English is another language) to discuss ideas. People included personal friends, Facebook 
friends, acquaintances and other general contributors to social interest websites. Most participants were also active in the online course discussion forums or followed these silently. These types of interaction extended but did not change their thinking as expressed in one student's interview: "but it's made me open to others' views. Instead of doing the 'my-way or the highway' I now go, this is okay, you think this, but I think that and it's okay" (To100).

These internal deliberations and social exchanges resulted in some behavioural changes. Having examined and consolidated their thinking on identity and their place at local and community levels, three participants prioritised and quietly started to build relationships with new immigrants, solo parents or the disadvantaged in their local communities. Another seven students recognised and acknowledged the ways in which they already contributed to build their local communities, in particular to address inequality. Two commented they wanted to understand and fix things they saw as 'wrong'. To do so required them to be actively looking for solutions. Studying in the first citizenship course had generated a lot of self and inner reflection, and consideration of further action.

\subsubsection{Global citizenship and responsibilities - 200-level}

The 200-level course examined a series of global encounters selected to raise students' awareness of diversity, ethical responsibility, rights and responsibilities in the wider world through connecting their own lives to global issues. The second section of the course centred on ethical responsiveness to challenges to people's civic rights. In group work, students then identified their options and opportunities for collective agency and action. Course activities and assessment included a group project, and a reflective essay on the course. Twenty-four of my research participants completed the second course and agreed to be interviewed again. The remaining seven of 31 participants either stopped studying for personal reasons $(n=4)$ or were unable to take the 200-level course as programme restrictions or timetabling required them to complete other courses.

I was interested in how the personal contexts of those in the 200-level course affected their consideration of wider issues. Analysis of transcripts and written work revealed citizenship ideas were referenced by these students through three different lenses - local responsibilities, global experiences, and where local responsibilities were interwoven with their global experiences.

Personal responsibilities and commitment to local communities played a key part in thinking about the impacts of globalisation for parents and younger students. A third of the 200-level students identified as having parental responsibilities with strong 
connections to local communities. Three were younger students (aged 18-25 years) whose limited experiences may have restricted their world view to local or national citizenship. However, the academic ideas presented were seen to have relevance to their own contexts. This is demonstrated in an email from one student explaining

"working alongside Māori, it made me sensitive to their world-views as well as that of others, I engaged with, i.e. retired elderly. By being culturally sensitive and viewing everyone as equal, I found value in my relationships and the connections I made" (LoEM200).

In turn, this student wrote that she continued to share with her sister the "amazing connections I made with the course to real life, [in her workplace] which was profound and enlightening" (LoEM200).

Others felt that discussing the tensions of globalisation's impacts on local contexts had helped to inform their decision-making, personal commitments and actions. This is illustrated in how focusing on global refugee crises in the course pushed two participants to become involved with refugees in their local communities. Others considered making more ethical choices in their purchases. A Māori student connected "hurtful and racists" (Na200) comments previously read in the 100-level discussion forum posts with ideas of refugees' loss of identity through displacement. She offered her explanation as an alternative interpretation - that racist comments reflected a person's feelings of uncertainty in claiming their place in Aotearoa/New Zealand. This thinking aligned with another non-Māori students' comments of discomfort with biculturalism - feeling as if they had invaded someone else's country.

Intentionally moving on-campus to live with international students created opportunities for a young student to connect with a wider range of people. This student felt she had become more open-minded though gathering and exchanging ideas on global citizenship with people in their dormitory from other cultures and countries. Living on-campus also enabled them to informally meet up with students from across disciplines. As explained in her interview, outside class, these across-course interactions and discussions had proven to be helpful:

even out of class - talking to people that you would see. And you would go like - hey we've got this class together so let's talk about it. What do you know we've got another class together; we can mingle those ideas with another class ideas. That was quite a cool experience to mingle those ideas (To200). 
Training as an online support person had also helped this person to make connections between their world and others "where you have to put aside your biases and thinking of the bigger picture" (To200).

Past experience proved to be both an additional resource and a complication. Mature students with extensive experience of living in other countries and cultures compared their knowledge and global experiences with the course ideas. Three students wanted to share their understandings with others or to challenge the conceptualisations of globalisation presented, but they lacked the opportunity to do so. One participant, who had delivered humanitarian aid in international crisis incidents, was uncomfortable with what they considered a narrow coverage of topics. Others, who described themselves as immigrants and travellers, felt all students would have benefited from looking at a wider presentation of cultural and political constructions of global rights and responsibilities. There was a sense that they believed their vast experience would have enriched other students' understanding of the issues.

Consideration of global responsibilities had extended and braided together threads of personal identity with communal obligations to reveal new awareness. Three younger students found their focus shifted as they connected their own experiences of living or travelling overseas to global responsibilities. The course brought new perspectives in thinking, revealing the deeper meanings held by others, and in turn, generating new and deeper meaning in these students. One, a Māori student, related Māori understandings of colonisation and disenfranchisement to the experiences of global refugees - in refugees' loss of identity, land and language. She recognised this was similar to the experiences of disenfranchised Māori; these losses impaired refugees' access to public services and opportunities to establish a sense of belonging as local or global citizens. Three participants also projected the findings of their reflections forward. They felt there were more opportunities available in global interactions for Māori and for a "more bicultural' (Ke200) Aotearoa/New Zealand to share the advances made in repairing the impacts of colonisation through showing manaakitanga, or respect, towards other indigenous cultures.

Examining global citizenship and reflecting on personal choices made one male student, uncomfortable with the lack of connection between his own values and global actions. Even though he identified as a global citizen and valued prioritising collective rights, he felt he had not done enough. At the course end he had reached his own position: in the interview he described global citizenship as "[first] us individuals of the world community and, then, prioritise the world community of humans as a collective group" (Ka200). He 
now felt his individual citizenship responsibilities and global values and actions needed to be woven together to help other people retain their cultures and rights.

At 200-level, participants were less inclined or lacked the opportunity to discuss global citizenship topics with others. However, those with broad cross-cultural experiences recognised the depth of knowledge they held as a result of their own global experiences and connections were a resource that others lacked.

\subsubsection{Changing circumstances}

Changes in personal contexts and priorities impacted on some 200-level students' study focus and thinking space. Three students struggled to engage meaningfully as their personal concerns for family and economic situations escalated during this semester, occupying both their reflexive thinking and time. One felt financially compelled to take on extra weekly shifts at work, leaving little time for study. Family crises meant three others re-prioritised study to make way for the demands of intense and emotional changes. After working through relationship breakdowns, two participants' lives and routines settled sufficiently so that they could re-focus more clearly on their studies. Parental responsibilities continued to impact on the time available for study.

The different pressures of life, advanced level study and changes in course assessment formats also affected opportunities for reflexive thinking. As one student explained in the 200-level interview, the change from presenting shorter critical reflections written in response to a reading - to writing an essay reduced her opportunity to work through ideas. In turn, this impacted on her confidence:

I'm into the quicker reflections and they are to me a really good size. And we had to get them down quickly and I really like that. I like that more than the way bigger assignments. I generally like it if something that size is broken into smaller chunks. It could be just the way my brain works. If something is really big you tend to go, my brain just gets muddled up, and I'm thinking: how can I get to narrow it down? And, it's just like it's too much to do, and to do it once. And then, then, I feel like there's only one way of doing it, and if you don't do it their way, then you're not going to do so good (Em200).

These findings made me aware that shifts in the personal and learning contexts of these 24 students had an impact on their reflexive thinking of citizenship and their identities. While some were able to engage deeply and make connections between the different facets of their lives and study, personal histories and citizenship concepts, others 
struggled to find the headspace to consider the course contents. This drew me to Archer's critical realist idea of reflexivity - lives lived in the present and rapidly undergoing change require the person to develop new ways of thinking (Archer, 2003) and Caetano's personal reflexivity as a characteristic that evolves through biographical change (Caetano, 2017a). While critical realism focuses on the external institutional or structural change, these people's social and personal circumstances also meant they were responding to the structural reality of their social situations consciously and unconsciously. They were expressing their agency in terms of the priorities and concerns they focused on, which I will discuss in Chapter 6.

\subsubsection{Active citizenship - 300-level}

The third-year/300-level course presented citizenship as an activity where people came together to discuss and prepare collective action to address issues within civic society. It drew on the previous themes of identity and belonging and global awareness and responsibilities. The course premised civic engagement as personal and communal reflection and action amongst citizens, and between citizens and the state or institutions. To benefit from the course, students had to apply their accumulated academic and civic knowledge to collaborate as citizens on a citizenship project. They needed to draw on personal and social resources, including their knowledge, to plan and implement an action. The students in this study were involved in projects such as building public awareness of mental health issues, petitioning government ministries for more funding for school lunches, and initiating environmental conservation projects.

Seven of my research participants completed the third course and a course project within the time frame of this study. Two others did not take the 300-level course as intended but spent the semester in international cross-cultural contexts and wished to remain in the participant pool. I was interested in how individuals used their extended knowledge of citizenship in the approaches they took to engage in their active citizenship projects. I wanted to understand - how do higher education students work through their course projects in circumstances where their values and priorities were likely to differ from others? Participants were at different stages in their completion of their studies and their lives, and in their personal and social contexts. This is reflected in their individualised stories of group work, which I share below.

Nan found working on a joint civic action to be inspiring and empowering. Nan was excited at the prospect of finishing her studies and actively seeking employment opportunities in organisations contributing to social change. The group project enabled Nan to develop team skills and experience in working with disparate groups towards a 
common goal. She felt this would be beneficial in her future employment. As team leader of four young first-year group members, she consciously worked hard to build her understanding of and engagement with these students. She adapted her thinking to fit with their main concerns in completing the course work. Indeed, Nan moderated her own large-scale environmental priorities in the group work to engage with a variety of local and regional government stakeholders and community groups to form a small land restoration project. She dispersed tensions within her group and with collaborators by quietly listening and identifying differences in priorities, to bring the group to consensus. Nan believed the group project was successfully completed due to cooperative planning and implementation. She saw the project as one of many small actions that would build towards wider environmental goals (which she valued) with everyone having a place to contribute. The teamwork illustrated to her that civic action was "not the power of one but working together" (Nan300).

This contrasted with Jem's autocratic approach. As the self-appointed team leader Jem used her high-achiever 'A-student' status as the main reason for taking control, deciding on the actions that needed to be taken, and directing the group project. She planned everything carefully, allocating tasks to team members, filling in when work was not up to her exacting standards and drew on her individualistic strengths. The team project brought together health community and business representatives in a public forum to discuss university students' concerns about their access to health facilities, and to look for possible solutions. Jem asserted herself to ensure the project outcome would be at the standard she set herself. She was proud of the work she had accomplished and proudly acknowledged the feedback from her fellow group members on the quality of the project. For Jem, the civic project was an opportunity for self-development.

For the less confident the project focus brought a disparate group of people together to form an inclusive group of people with a common cause. Nell (who was consciously and subconsciously aware of what people thought of her) felt unjudged, accepted and comfortable in the group she belonged to as they worked on a submission to a parliamentary inquiry on public health services. They worked cooperatively without the need for a leader to complete their project. The group processes and support of each other, made Nell's experience enjoyable and built her confidence. Nell attributed the success of the project to the whole group. She gained confidence and learnt a new way to promote a cause, which Nell recognised in the interview was an opportunity to use her voice and experience to make a difference. Despite her feelings that the group's views, and hence their voice, had been dismissed in their submission to a government body, 
Nell had been empowered through working with others who understood her - her primary value. This experience had created a new passion in Nell towards planning positive action projects. As a consequence, Nell had extended her social networks and was continuing to work on her life projects.

Four other participants' experiences of practicing engagement were less successful (these are discussed in more detail in Chapter 5). A perceived lack of commitment by other students to the task impacted on Sage's engagement. Groups were allocated by a tutor and group members needed to work together to decide on a topic and an action. Sage had a full final-year workload and little time available. Delays in group members making contact, and what she interpreted as their lack of interest in civic action, meant Sage took a functional approach to the group work doing just what necessary to pass the course. Sage's focus continued to be on her family and close friends. While Sage understood individuals could become the catalyst for change in society, she recognised that issues of inequality, instability and disempowerment holding her and her friends back, were not a priority for her group members. In the interview, she expressed her frustration in the realisation that people like herself were not free to engage as citizens in society and the "injustice of it just drives me crazy" (Sage300) - Sage distanced herself from her peers and the possibility of social action.

Shazz's priority during the semester was her family and dealing with a family issue. The choice of the group work project - creating inclusive recreational environments - fitted within her priorities. This allowed her to engage with the task and provided a means to express her concerns. However, the emotional stakes of dealing with an overlap between the family issue and the project focus were high. This left her feeling that as a citizen "I can't save the world at the moment" (Shazz300). Consequently, she limited her interactions with the group and stakeholders. She saw herself as peripheral to the participatory action, explaining in the interview she was a "citizen sitting on the side-line, waiting until the conditions are right" (Shazz300). As her circumstances left her dipping in and out of group work, these reflexive moments left her frustrated - a feeling echoed in her inability to manage other situations outside her control.

Karen also found the mismatch in priorities of group members impacted on her opportunity for involvement in the civic action. Karen was in the final semester of study but was working part-time for an education provider. In starting their group work, Karen's group members differed in opinions on what their project topic and focus for action should be and continued to disagree. Karen applied her 200-level course knowledge, which examined how information was framed by the different stakeholders, to look at her group 
members different approaches to working towards a specific goal. In her view - "the same result can be achieved from different directions" (Karen300). Unfortunately, Karen found the group work schedules suited those members with fewer commitments. Group meetings often clashed with Karen's family's priorities and constrained her ability to engage with the group. Karen was very conscious of her low level of input into the project, but felt the group struggled to respect the differences in individual circumstances. Consequently, despite her personal view that the project could be achieved by disparate parties, her team did not complete their participatory action project.

Echoing her earlier experiences of participatory action where she did not see an immediate outcome, Elsie also felt little satisfaction from her participation in the group work. She had little interest in discussing the project and the group process. However, she had gained analysis tools from the course materials that would help her in the future. Elsie felt more informed on how to frame a project so she could promote her point of view. She believed making an action materialise was her responsibility and recent "experiences of structural inequality and how it plays out" (Elsie300) in her own life and community were now helpful to her as she sought work in the wider community. She was mentally planning a future project to tell the stories of marginalised people in society.

The two participants who did not complete the 300-level course still saw opportunities for collective action as a result of earlier course preparation. Kane, acting on his identification as a global citizen, took a work sabbatical to study international relations full-time in an overseas university. He was active in engaging with his peers, although very conscious of how his work in Aotearoa/New Zealand civil service and views differed from those people currently around him. While he would seek his peer's views, he was reluctant to share his own views in case he offended people. However, his engagement in his current courses and listening to culturally different peers had built his stock of knowledge. The outcome of this study excursion was he was looking for opportunities to take part in global participatory action as a career shift.

Study in the earlier citizenship courses had developed Deedee's understanding of her rights and had empowered her. Deedee, who was living overseas and had just given birth to her first child, decided to reduce her workload to part-time study and did not take the final citizenship course at this stage. Her child is by birth-right a citizen of the country she is living in. Having a child had opened up interactions with casual acquaintances in her community and provided a focus for learning more about the constraints and possibilities around her situation. She held intense discussions with expatriates on Facebook as she asked questions about how others had dealt with cultural and political 
restrictions on their movements as parents. Nevertheless, Deedee would not discuss her concerns with her partner out of fear of being misunderstood. However, being proactive in seeking solutions to her current context had also allowed her to revisit her environmental concerns and choices, and she had taken part in a small protest action.

In this section I have presented findings on the social interactions, ideas and values the participants in this study referenced in their social interactions and conversations over the period of study. When faced with planning and working collaboratively on a civic action task or acting alone as a global citizenship, there were considerable differences in approaches in dealing with or responding to their interactions with structures and other people's values.

\subsection{Chapter summary}

In this chapter, I have introduced the 31 participants, their contexts, external interactions and conversations over the different levels of participation in the three citizenship courses. I found participants' attitudes towards the required courses, as restraining or enabling, varied as did their appreciation of the opportunity for discussion on the course topics. The courses initiated some deep and on-going internalised reflections and externalised conversations on personal and others' opinions, obligations and responsibilities. Experiences of participatory action were impacted by personal and group values and commitments. These shaped the projects and their success. Finally, the pursuit of projects which aligned with values empowered and encouraged possibilities for further action. The next chapter presents a synthesis of findings from the nine 300-level participants' responses to research questions on the reflexive processes that learners used to make sense of the citizenship ideas that they had gathered. 


\section{Chapter 5. Participant vignettes and analysis}

\subsection{Introduction}

Understanding diverse higher education students' reflexive considerations of the enablements and constraints of their circumstances, values and concerns provides an indication of their agency or freedom to act (Archer, 2000) on new understandings. In this chapter, I present my findings from research with the nine 300-level participants. I conducted three semi-structured interviews (between 48-80 minutes each interview) with each of them, then transcribed and analysed all the interviews (described in 
Table 5.1 below). I also collected and analysed their coursework and discussion forum contributions. Of the nine stories told in this chapter only one is male. The remaining male participants did not complete the final course in the data collection period due to work, personal commitments, or the staggered nature of their overall progress through their degree. Such breaks in distance study are common, in particular for males who feel social pressure to financially provide for their families (Brown et al., 2015; Kahu et al., 2014; Poskitt et al., 2011). In part one, participants' stories are followed with the analysis of their internal conversations and external deliberations in considering citizenship concepts. 
Table 5.1 Participant completion and interview stages

\begin{tabular}{|c|c|c|c|c|c|}
\hline Participant & $\begin{array}{l}2017 \\
\text { Semester } \\
1\end{array}$ & $\begin{array}{l}2017 \\
\text { Semester } \\
2\end{array}$ & $\begin{array}{l}2018 \\
\text { Semester } \\
1\end{array}$ & $\begin{array}{c}2018 \\
\text { Semester } \\
2\end{array}$ & $\begin{array}{l}\text { Number } \\
\text { of } \\
\text { Interviews }\end{array}$ \\
\hline Nan & $\begin{array}{c}\text { Interview } \\
1\end{array}$ & $\begin{array}{c}\text { Interview } \\
2\end{array}$ & $\begin{array}{c}\text { Interview } \\
3\end{array}$ & $\begin{array}{c}\text { Interview } \\
4^{*}\end{array}$ & 4 \\
\hline Sage & 1 & 2 & 3 & & 3 \\
\hline Kane & 1 & 2 & & 3 & 3 \\
\hline Nell & 1 & 2 & & 3 & 3 \\
\hline Shazz & 1 & 2 & & 3 & 3 \\
\hline Deedee & 1 & 2 & & 3 & 3 \\
\hline Elsie & 1 & & 2 & 3 & 3 \\
\hline Karen & & 1 & 2 & 3 & 3 \\
\hline Jem & & 1 & 2 & 3 & 3 \\
\hline \multicolumn{6}{|c|}{$\begin{array}{l}\text { * A fourth, after-degree interview was held with Nan but due to timing } \\
\text { constraints was not able to be held with the other } 8 \text { participants }\end{array}$} \\
\hline
\end{tabular}

\section{Karen}

The complexity of Karen's life was reflected in the complexity of her reflexive processes and commitments. In her interview, Karen described herself a busy stay-at-home, mum of four, part-time school volunteer, active in her church and local community, living in a provincial town. She had decided she might become a teacher and started her part-time BA studies. Her plan was to continue on to a postgraduate certificate. Her own life had been shaped by family and family experiences, "your own kids shape you" (Karen100). Karen valued and prioritised her family, her children, modelling good relationships, treating people fairly, and commitment to her faith and church community.

Karen believed that people's actions and values should match. She questioned what she saw as a mismatch in Aotearoa/New Zealand, between individuals' concerns for themselves and the environment, and concerns for other people and their voting on issues. Before commenting on the wider world, she felt "[w]e need to look after our own backyard" (Karen100). Karen thought she was "not a bad citizen", stating that she sat on jury service. On the other hand, she felt she was not overtly visible in standing up for her 
community as her involvement in an earlier active protest had "left me feeling uncomfortable" (Karen100). Karen was a talker, listener and sharer in all her communities. A family issue, during the 200-level course, meant Karen concentrated on guiding her child through a difficult time. More importantly, this was a situation where Karen felt powerless as a parent. Although the situation had not been resolved, by the 300-level interview Karen had moved on to focus on wider concerns. To add to her skills and resources she had added a Te reo Māori (language) course into her busy schedule and increased her work hours.

The constraints of adding the citizenship courses had left her conflicted between her personal goals and social values. For Karen, the citizenship courses created a sense of belonging in the social science degree but, as explained in the first interview, were "a bit of a pain in the backside for me" (Karen100). However, the first course was enabling in that it helped her to develop more confidence in her identity and in building relationships. Exploring identity roles helped to push Karen beyond using the white-female-European descriptors provided on surveys and official documents to use other characteristics of her identity. In the third interview she described herself as a citizen who is aware of her community and knew what was required to bring about change:

far more aware of the impact of what is going on around me than what I was before, and aware that if we want to make changes then you are going to have to find a like-minded people you are motivated to work with, to start making those changes. But it is not going to be an easy process, it is not going to be a quick process. It is going to need to be long term and sustained to make any change (Karen300).

Working logically through ideas and concerns in the courses, Karen developed the reasonings behind and extended her understandings of her identity. In the first course the critical reflections and examination of her beliefs inspired and directed her thinking. She recognised a tension between the course's bicultural context and her own reference points for engaging in the material. There was a critical change in her understanding of bicultural values and historic actions over the citizenship courses. Discovering the gaps between the structural assumptions underlying political narratives used to explain the national identity (for example, 'a clean green New Zealand' and a country that was 'aging successfully') and her experiences of the reality of living in her local community had challenged her. The disparity alarmed her. 
This understanding of the logic behind and consequences of actions was demonstrated in Karen's written comments on citizenship as a combination of national and individuals' global responsibilities; she saw local and global actions as entwined:

New Zealand is a melting pot of cultures, ethnicities, backgrounds and country of origin. The population demographic means we are a global country. The concept of global citizenship to me is the understanding that we each have a place in this world, it may feel that we only live local, but globalisation effects are everywhere.... We need to stand up as global citizens and demand changes, just because we are from a geographically isolated nation does not excuse apathy. Actions taken here can have an impact on the other side of the world; an internet movement created in support of a population can inform and encourage support (Karen300WW)

Summarising the overall course effects on her citizenship in the third interview, Karen identified the turbulence of her thinking, on-going questioning, and the need for action:

it has brought a change in the way that I think of myself as a citizen ... I think it has changed my awareness of what we need to do to make a difference. To bring to the front of thinking - what it does it mean to be a part of this world? and what do we need to do? We can't all just sit in our little boxes .... We need to get out and make that change. We need to be aware of what's happening in our world and we need to be aware of what's happening in our community, our world and our society and close by, as it all impacts on the whole (Karen300).

Karen's agency was entwined with her family and community values and these are her primary commitments. In the interview at the course conclusion, her thoughts on citizenship were "our backyard is global. We are connected to the whole world and an impact here has impact elsewhere, and things happening elsewhere have an impact on what is happening in New Zealand too" (Karen300). She did not see herself as agentic or proactive, but that she acted on her values to build relationships in her family and communities. In talking about the future, she pondered

I certainly want to be involved in my community... in teaching I will be involved in my community ... And how we are raising our children... I would like to think that if we could make some community changes that would be really cool (Karen300). 
Karen's plans to help develop her communities were an incentive to completing a postgraduate qualification.

\section{Jem}

Jem's focus on her personal achievements contrasted with Karen's complex social commitments. Jem was a full-time BA student. She described herself as single, an intelligent high-achieving student, active in the community, creative and with strong options based on her life experience. Her privileged childhood had provided access to educational activities and life-style choices, allowing her to develop into a confident, skilled person. Goal-orientated and motivated, prior to her current studies Jem had created and successful run her own businesses. Jem's goals were to complete her undergraduate and postgraduate study to become an advocate for people who cannot speak for themselves.

Jem's confidence and strong views meant she spoke freely in social situations. At the start of the citizenship courses, Jem identified many threads in her identity as a Pākehā. She identified dualities and contradictions in her identity - from financially and socially privileged as a young child, to becoming a poor and homeless teenager, yet still a compliant taxpayer and worker. A child who dealt with family breakups and as an adultchild caring for her siblings. She considered herself to be a self-reflective, knowledgeable and idealistic critical thinker, a responsible and active citizen in advocating for the underdog; where "[my] voice is the most powerful tool I possess" (Jem100). Jem believed people gain citizenship in their communities by being active; membership is conditional on acting in the right manner.

Jem had little tolerance for, and was confident to confront, injustice or unfairness when witnessed in the community regardless of her own safety. The goal of her action was to make the community better for all. Engaging with the courses had strengthened Jem's position on her citizenship. At the 300-level interview, she defined citizenship "[as] engaging in the community, putting yourself out there. Now you have information, you put it into practice" (Jem300). She had gained tools to help advocate and discuss issues with people without being confrontational. She continued to see herself as an active citizen - contributing to food pantries, engaged with her local community, promoting wellness, empowering and giving to people sleeping rough. However, her attitude was changing

before I saw it as charity, but it's not ... about trying to improve our community and the way it is... not always feeling good... it's about people trying to make 
their little part of the world a better place and recognising that that is very empowering (Jem300).

Jem valued truthfulness in confronting ideas which did not match her own. She felt it important to challenge what was said about her country's history and her own knowledge of Māori, all the same she had not anticipated resistance to her interpretation of truth. She shared her views and was surprised to find that some of her ideas on the course discussion forums were seen as racist or condescending. As she wrote on the discussion forum, she felt "slammed by black \& white responses" (Jem 100DF) and disengaged for a while. In a period of self-examination, she identified that as a child she had integrated her parent's racism into her own beliefs and had now begun to recognise her own views as racist. Tension existed between Jem's value of truthfulness and her approach to social interactions to achieve her goals, but she was not overly concerned about this tension.

\section{Nan}

Nan, a solo mother, competed her fulltime study on her BA during the time of this research project. Born in New Zealand, she identified as Māori but grew up in another country. She had a strong sense of belonging and kōtahitanga/unity in her Aotearoa/New Zealand identity. She valued "sharing and caring" (Nan100) for the land and people, and the heritage of her parents fostering children during her own childhood. Nan lived rurally, was interested in the welfare of people and society and looked out for opportunities to improve her local community. In the first interview she described herself as optimistic looking for solutions to problems. For her as "tangata whenua [indigenous to the land], kaitiakitanga [care for the environment and its people ]" are part of her being, her identity and "the future of my moko [children and grandchildren] and future generations" (Nan100). She was concerned with how Māori were presented and present themselves and with how Aotearoa/New Zealand was presented and presents itself to the world.

Nan's views of herself and place in society were strengthened rather than changed over the citizenship courses. Nan's identity descriptors changed from roles and national identity to recognition of her personal citizenship characteristics. She discovered she was a team builder and could keep people focused on the task at hand. Her cultural identity had not changed, but she believed that through her learning her future self could make a difference as a citizen. As she explained at the third interview:

Success to me means making a difference. Success to me doesn't mean having two houses, ten cars, some boats and stuff. Success to me means making a difference, feeling good about some positive changes for people. 
Lifting people, empowering people. Empowering society, ... being part of change. ... I'm also interested in Māori development too. ... Everything, every job that you do, well I hope for me, every job I do will have some, will contribute to some change for the better (Nan300).

Nan took and responded to opportunities as they arose to look outward. Initially, Nan expressed her fears of loss of culture in examining broad concepts of citizenship, explaining "we need to look at our bicultural picture first... and then we can work on multicultural" (Nan100). She saw Aotearoa/New Zealand as a "host country" as "Māori are tolerant of other people's differences and our own" (Nan100). Nan spoke of tolerance in listening to people, to hear where they were coming from. She was hurt reading racist posts on the discussion forum. To try to understand why a person would write these things she talked to refugees and new immigrants about their experience. She read posts from other racists on social media. By stepping into other lives, Nan heard their anger as hurt and she located it as loss of tūrangawaewae/having a place to stand, something she could identify with.

Nan's views shifted over the courses to recognise Aotearoa/New Zealand as having a bicultural foundation, but a multicultural population open to refugees - "there is room here for more with the same values and care for the land" (Nan300.) Being the elder, with strong values and ideas, in an active participation group with younger students meant Nan adjusted her expectations to match the situation - to learn about "the other side" (Nan300). In doing so, she recognised she had learnt a lot of tolerance, to listen, respect and understand. She had learnt other people could be narrow-minded, but needed to learn where they fit, to understand themselves, and others and their values. To think about walking "in others' shoes”, experiencing without judgment or bias "learning how to take other people with you" (Nan300). For Nan, there was reconciliation in understanding that the comments made by others often had deeper, unexamined origins that people were not aware of.

Citizenship had taken on new meanings for Nan. Nan's national identity and self-identity were aligned with empowering people and communities to care for the land in meaningful ways for future generations. She recognised that active citizenship occurred through working together, not through the power of one person. Being a responsive citizen meant combining solutions to solve problems and allowed Nan to involve her passions and stick to her values. Nan saw herself as civically engaged, contributing to change in any way possible. Participation in the citizenship courses had given her the confidence to use her 
voice (including in online forums) and to take responsibility to act at community level to bring about change which would have an effect from local to global level.

\section{Sage}

Sage described herself as a life-long learner with computing and therapy qualifications and a single mother of four, now adult, children. She had started her degree 15 years previously with goals of "wanting to grow children who were conscious and able to make good decisions" (Sage100), to broaden her own perspectives and to meet her love of learning. As a single mother she struggled with getting the basics of life - feeding and caring for her family with little time to consider other things. In the first interview she described being "[c]onsumed by the smallest details" (Sage100) to make ends meet. Sage considered herself semi-political, conscious of inequality, instability and disempowerment, and mindful of the [previous] government's focus on individualism. She felt she needed to be more active, to use her voice and solo mother experiences to bring about change in the community. During the research, her circumstances changed with a new partner, completion of her studies, and looking for employment.

As a citizen, if Sage sees something wrong, she wants to understand it and fix it. To do so, she believed a person needed to be active about finding solutions. However, being responsive was tied to economic security. She wanted a more inclusive Aotearoa/New Zealand that recognised the strengths of all forms of diversity, in particular the poor. Exploring the concepts of identity and belonging in the 100-level course had impacted on Sage's consideration of future opportunities. Sage recognised and articulated the civic consequences of her own position. In the first interview, she explained she believed supporting individuals benefits society, but that people needed to learn how to use their voice and to empower each other.

[K]ids don't know what they want to do, and they are feeling just so disempowered and trying to talk with them and trying to encourage - get them involved and saying - what can we do? We've got no power. So, I push talk to them about getting out there and having a voice (Sage100).

Sage believed political voice and political decision-making affected individuals and citizens, and their ability to participate in society. If individuals could "get it right at home" then society could fix global issues. Key to her thinking in the second interview was that active citizens needed a secure base to work from, a "collective consciousness supporting the mind and spirit was needed in the absence of hope and religion" (Sage200). In the 200-level interview, Sage's enthusiasm from the previous interview 
was noticeably absent. Where she previously spoke about her dreams and possibilities for the future, the lack of freedom due to her personal situation had dampened her dialogue. By the 300-level interview, Sage had moved from owning ideas to just thinking about whether or not she had passed the course. In the final interview, there appeared to be a disjuncture between her own beliefs and actions in the citizenship course "you just get what you need to, to learn and understand the core concept of engagement with the community" (Sage300).

In an email to me after completing the course and knowing she had finished her degree, Sage summed up her change over time as:

I guess one of the key things I learnt was the idea that people need to learn the skills of what good citizenship is. That it is important to instill [sic] these ideas in our children... With the world becoming more and more connected (yet less connected to community) and with technology changing so many things these skills become increasingly important... Learning how to engage, question and participate with the world around us rather than the focus always being about how the world impacts on us (Sage300EM).

Although limited by finances and their associated social circumstances, Sage did not feel she lacked agency altogether, but she was constrained in the extent of what she could accomplish. In the first course Sage had demonstrated her resolve. During the 2017 Aotearoa/New Zealand political elections she discussed voting options with her family and friends, pushing them to vote on election day. Describing this action in her final reflective assignment, she wrote that in doing so, she had been fulfilling her own dreams of "sharing my knowledge and giving back to society. ... to help move us to a place where growing children is valued by our society rather than just another added pressure in people's lives" (SageWW300).

At the end of her degree (which she completed after fifteen years of part-time study), Sage saw herself as a citizen "as someone who's involved but who would like to be more involved" (Sage300).

\section{Nell}

Nell was living in a provincial city when I met her. At 16 she had left a broken home and gained an international studies diploma at a college that she now described as "accepting and understanding" (Nell100) of her. Now, in her mid-twenties and part-way through her BA, acceptance and being understood were important values for Nell, along with 
prioritising study, completing her qualifications, and maintaining relationships with family and friends. In her first interview, she felt she could "describe myself in different ways according to the situation, as I feel no one is really interested" (Nell100). Difference and acceptance as themes came throughout Nell's conversations - in considering citizenship she thought "about Asian people moving to New Zealand and being poorly treated - it was something that I could relate to and affected me" (Nell100). Nell had anxiety and depression and often felt misunderstood by those who have not experienced such illness.

By the time of her 300-level interview, Nell had moved cities to be with her fiancé and completed the final course of her degree. She appeared more confident, settled and outward focused. She was looking for permanent employment. Nell had joined and volunteered for a political party, "it's like it is its own community, everyone has similar thinking" (Nell300) and was promoting mental health awareness. She was active in online discussion forums debating issues important to her. Nell had participated in her group's social action (raising political awareness of anxiety as a mental health issue) and, even though she felt their voices had not been heard, she now knew how to construct a form of social action.

Nell saw herself as strongly opinionated about critical issues. The citizenship courses allowed her to draw from her personal, study and life experiences to critique and challenge ideas. In the 100-level interview, she saw citizenship as linked to national identity, to mutual sharing, and openness to others in the community - attributing this view to earlier cross-cultural experiences. This perspective was carried across time, as explained in the second and third interviews. She felt focusing on individualism, rather than collectivism, undercut indigenous rights and collective responsibility; however, citizenship's locality was engrained in her. As she explained in two interviews:

I've always tried to keep an open mind in relation to citizenship and I do tend to default back to citizenship as where you live all the time. ... One view [is] ‘'m a citizen of New Zealand'. I'm not a citizen of the world or whatever. People can be very, like, nationalist at times (Nell200).

And

I think I haven't really changed now. It's really hard to change the meaning of the designation in my mind, because whenever you hear the word it goes straight to having a New Zealand passport, and that is more often where we come across it, so it is hard to separate the government, the legal from it (Nell300). 
Having completed her group project, Nell felt strongly the voices of all citizens should be heard. However, in the last interview she felt "people are not listening to those of us who are having these problems. ... there is a disconnect between reality and the rules...[The] reality is citizens are disempowered by structures and power" (Nell300).

Nell had now connected ideas of citizenship with agency. She saw life-skills taught in childhood as preparation for adulthood and citizenship. She believed parental wealth and actions can impinge on future agency by removing children from the practical skills of citizenship and the social tasks expected of them. Nell's social commentary connected the simple tasks of living - knowing how to mow a lawn, clean an oven, and get a driver's licence - as tasks of active citizenship. She recognised her own agency was restricted by her mental health, but her social consciousness had grown to the extent that she had worked out strategies to get around this.

\section{Shazz}

Shazz, a full-time mother to two young children, lived in a large city with her partner of Pacific Peoples heritage. As a teenager growing up in 1990s Aotearoa/New Zealand she felt like she had invaded someone else's country. She wanted to be a part of Māori culture and valued as a global citizen. She now saw herself as "a white New Zealander" (Shazz100) with European heritage and a part of multicultural New Zealand. She valued family, having a voice, time with her close-knit extended European immigrant family, raising her children and living authentically.

As she explained in her first interview, as a new student, Shazz valued engaging with people that she would not have encountered earlier outside of the citizenship course. In the first interview, she elaborated on her feeling that

my world is very small because, I think because the kids are young. Look, it's got very small because I think my focus is so much on them and what's happening in our little world ... I am a citizen in that I am really interested in people and I want to understand people and people's point of view a lot more than I used to (Shazz100).

Exploring identity and belonging had provided Shazz with an understanding of her own tūrangawaewae/place to stand and cemented her idea of herself as a citizen in a relaxed and accepting multicultural community. She wanted "to just find a voice within that [member of a country, a community], a respectful voice" (Shazz100). She felt talking about citizenship and global citizenship in the 200-level course was better than ignoring 
it, telling me "even talking about it is good, and even debating it and having those uncomfortable, you know having that tension and acknowledging the tension, rather than shying away from it' (Shazz200). Showing manaakitanga/respect and hospitality towards refugees she saw as a right and a responsibility. This thought had developed further, in her now being conscious of the ways politics at a local level impacted on individual and global affairs. She felt issues that looked big in Aotearoa/New Zealand were small when compared with global issues.

Over the time of the research project, Shazz's circumstances changed with her children's growth, school attendance and other activities. In the 200-level interview Shazz was engaged in local activities helping to build her diverse community. She was on a local community board and looking at making her suburb more refugee friendly. At the 300 level interview Shazz was stressed as financial and personal pressures mounted. She was trying to balance a new full-time job, study and difficulties with her school-aged child. Her focus was on her immediate circumstances and the things that needed dealing with.

At the conclusion of her 300-level citizenship course, Shazz described herself in the interview as "not a good citizen at the moment" (Shazz300). Her world had shrunk as she struggled to focus on solving personal issues. She felt like a citizen on the side-line - with knowledge but in no position to work with it.

Sitting in the middle as a citizen, not doing anything huge to make changes, but at the same time trying to make sure that my kids are as healthy, socially aware as they can be. That's kind of my little gift to the world, that's all I can do right now (Shazz300).

Shazz started with enthusiasm - the citizenship courses had allowed her to explore and own her identity, this contrasted with her experience of growing up and feeling an alien. She could see opportunities to act as a citizen in every corner of her life and acted where she could. However, over time, her immediate family circumstances and full-time work become higher priorities and consequently her focus rather than her studies.

\section{Elsie}

Elsie lived rurally in an intentional community with her partner and children. The community described itself as a planned residential community with a higher degree of teamwork than other communities. Elsie held qualifications in alternative therapies. Her values lay in her community's beliefs and connections to environmental sustainability and land rehabilitation. She had a strong sense of self, place and connection to nature 
and others. She talked about belonging in community as "a rich way of living" (Elsie100). Elsie identified herself as a global citizen as national citizenship never "resonates with $m e "$ (Elsie100). Past activism in protest marches had left her disillusioned due to the lack of results of these actions.

Elsie's relationship broke-up during the 200-level citizenship course. Community members' attitudes towards her changed based on her relationship status. Her focus and direction moved towards providing for herself and children. Once firmly engrained in and attached to her community, Elsie's horizons lifted as personal circumstances and the limitations of employment opportunities within her community were realised. A sense of displacement and uncertainty in the final interview was not present in earlier interviews.

In her writings, Elsie viewed citizenship as her "membership of a country" with rights and responsibilities and "membership within an intentional community with rights and privileges"; citizenship provided access to resources (ElsieWW100). She described participation

in the community I live in decisions are made by full consensus. Rather than a democratic model. We ... have developed a strong internal 'counter' culture [in the] ... absence of common national heritage and a more communal approach to wealth and land management (ElsieWW100).

Emotional belonging in her community was important to her. There communication and relationships were fostered in "sharing circles" (ElsieWW100) which allowed people to disclose their emotions and feelings "in supportive, non-judgmental space" (ElsieWW100).

Elsie wrote about citizenship as "collective symbolism" which should be centred on "ecological sustainability, language" (ElsieWW100), later adding in "Māori cultural symbols" (ElsieWW200), to "promote a deeper sense of meaning and belonging for a group of super diverse people" (ElsieWW100). Elsie wrote of citizenship as identity and belonging as "idealistic... It ignores dependence on imported resources to maintain a certain lifestyle infrastructure necessary to self-sustain communities" (ElsieWW100). In her course assessment, Elsie disagreed with the 200-level presentation of global citizenship as "it is possible to be a member of nation state but not be treated equally as a citizen e.g. indigenous right. There are many ways to frame global citizenship depending on the agenda and cultural assumptions of the person looking" (Elsie200WW). 
In order to become global citizens, Elsie believed society needed to deal with issues of discrimination, fairness and injustice at local levels first. Elsie's argument centred on power and who holds power, arguing in her assignment how it can limit "rights to selfdetermination and nation-state responsibilities to protect and respect indigenous peoples, their territories and their resources" (Elsie200WW).

In the interview at the end of the three courses, Elsie's position had softened. Rather than being critical of society's actions she acknowledged herself as a member of a wider community of citizens attempting to change or shift the prevailing culture. She had participated in a citizenship project with fellow students and developed an understanding of how information is framed and "framing [is used] to manipulate people, how to get your cause seen in the right way" (Elsie300).

\section{Kane}

A part-time student, Kane began the citizenship courses part-way through his BA. Fulltime public sector employment kept him involved in the wider community. Kane grew up in an intentional community committed to building family relationships and engaging with environmental values. He has lived in New Zealand and overseas. He valued his family and upbringing, community and working with people. Although Kane felt responsible for the earth and its environment, he did not believe he acted on environmental values. $\mathrm{He}$ held his siblings and their work in indigenous rights in high regard. Kane took a work sabbatical to study full-time in an overseas university during the time of the research project. He returned to New Zealand, his regular work and study at the end of his sabbatical.

Kane's thoughts of citizenship at 100- and 200-level fluctuated in response to exposure to new understandings. His post-200-level sabbatical experience allowed him to see how polarised concepts of citizenship can be held by others compared with his own situation. As explained in the interview:

I feel like back home we have a really strong, whether we realise it or not, a strong belief in some responsibility to the world at large and I think people here do as well, but I think they don't realise as well the impact that they have on the rest of the world, potentially. Perhaps more responsibility than they realise here (Kane300).

Kane's ideas on his identity, citizenship, and rights and responsibilities had consolidated over time. He saw New Zealand as a place of melding of cultures (where birth-right 
citizenship is a privilege) where citizens have an opportunity and obligation to voice concerns for others' rights - particularly when their citizenship rights were at stake.

His values were tied to views of global citizenship and his New Zealand national identity. This, he believed, recognised everyone had a global right to access a country to live in. He discovered he could be active in ensuring people have a place to which they belong. He plans to work in the United Nations to ensure individual rights are upheld,

but within the framework of global community rights...working for an organisation with worldwide reach and involvement ... to deal with people as people. ...but if it's your job to be part of an international organisation on which, that is, it is the base line for dealing with every people of every culture and on every issue, huge issues. That's very attractive (Kane300).

Kane consulted his siblings for their thoughts after deliberating on issues or disparities between ideas presented in the course and reality. He acknowledged prior to studying citizenship, his internal conversations had not resulted in actions. Participation in the citizenship courses had provided a focus for action. His sabbatical was part of that action. Kane was agentic ally thinking about the present and planning his future direction as a global citizen. He was exploring wider possibilities for employment in the United Nations upon completion of his studies.

For Kane, the stimulation of thinking about what opportunities for citizenship provided, and his discussions with his siblings, were considered deeply in his internal conversations. These led to a change in future direction. Widest possibilities were being considered and Kane was actively planning his role in global citizenship to bridge the gaps he saw between his ideals and reality.

\section{Deedee}

The 100-level citizenship course was Deedee's first course at university. Deedee described herself as "a semi-nomadic welfare child of an alcoholic mother" (Deedee100). She had been financially independent since 16, with a successful career running businesses and teams in New Zealand. She was now living overseas in her partner's country and home city, with limited ability to communicate in the local language. She had discovered as a foreign resident without a university degree she was not employable, despite her extensive work experience. She had no income or rights as a female within the local culture and was dependent on her parents-in-law for accommodation and language learning. Her on-going identity crisis had shifted with an unplanned pregnancy. 
Being a student provided Deedee with an identity separate to that of wife of her partner. Her previous identity and values aligned with her work - "honesty and hard work". Now her goals were to communicate better, to look after her personal health and gain access to medical services, and to build her relationship with her partner as she explained in the interview "I don't have examples of men as role models or influences in my life" (Deedee100). Deedee held environmental responsibility as a high value but was struggling with how to enact this with limited finances in a country where the environment was not valued. This was an emotional struggle for her.

Deedee completed the first two citizenship courses and then reduced her studies following the birth of her child. She and her partner had moved to their own apartment. Deedee had discovered she had no legal right as a mother to take her child out of the country. To do so, she required a letter of permission from her husband to travel with her child.

The citizenship course had provided a focus for Deedee to examine her past, present and future. Deedee was uncomfortable with her lack of knowledge about her bicultural identity - wanting to wear a pounamu (Māori greenstone carving) to recognise her whakapapa/ancestry - but uncertain whether she could as she did not know her Māori genealogy. Likewise, she recognised the cultural differences and tensions of place and relationships were very real in her daily life as she witnessed examples of classism, sexism and racism in the local culture. Examples of injustice and unfairness aroused highly emotional responses in her. She battled with the concept of ontological security (as a sense of order and continuity) presented in the course, due to its lack of existence in her past and present circumstance, and her uncertainty about the future.

While Deedee was adapting to cultural norms, she held fears about her lack of agency for herself and her child. Her change in personal circumstances meant she looked at international conflicts with a different perspective, with a closer understanding of the plight of refugees and displaced peoples. At the final interview, she saw herself as a global citizen - before "I was from New Zealand now I would say I am a citizen, a global citizen, for sure" (Deedee300), a global citizen with more awareness of what was happening in Aotearoa/New Zealand.

Deedee compared her loss of rights in her current country with her home country's generosity in giving immigrants' rights to vote, and access to public services and employment. In the final interview, although struggling with her immediate family having different citizenship status and rights of residency, Deedee now had a sense of belonging 
in her child's birth country. Having a local child had opened access to opportunities to uphold her values, including joining a protest walk for the environment.

Deedee identified a tight connection between her internal conversations, lack of financial dependence and opportunity to act. The focus of these deliberations was on the constraints of her citizenship status and the lack of opportunities to connect into her local community due to her limited command of the language. Notwithstanding that, while having a child had constraints on her moving between countries, the presence of her child had provided a link to local social life. Deedee now understood her loss of rights and freedom to move between her home country and place of residence. She was mulling over the possibilities to counter these restrictions.

\subsection{Archer's mental activities}

In the previous section I introduced the nine participants, their contexts, external interactions and conversations during participation in the citizenship courses. I now present my synthesis of findings in answer to the research questions on the nature of the inner and external reflexive processes that learners used to make sense of their developing citizenship knowledge.

I used Archer's list of mental activities (Archer, 2007) directly with participants (Table 5.2 and Appendix D). This approach follows a small number of other researchers who have applied Archer's mode of reflexivity, in biographical reflexivity studies (Caetano, 2017b; Chalari, 2013; Hung \& Appleton, 2016) and transitions to higher education (Baker, 2019; Bovill, 2012; Dyke et al., 2012; Kahn, 2017; Matthews, 2017). As discussed in Chapter 2.2.6, the ten mental activities were derived from interview transcripts of members of a UK working class community, and later UK undergraduate sociology students, when Archer tested for the presence of her modes of reflexivity. 
Table 5.2 Summarised from Archer's (2007) list of mental activities

\begin{tabular}{|l|l|}
\hline \multicolumn{1}{|c|}{$\begin{array}{c}\text { Archer's Mental } \\
\text { Activities: }\end{array}$} & \multicolumn{1}{c|}{ Definition } \\
\hline Planning & $\begin{array}{l}\text { Mental preparation in the act or process of making a plan, course of } \\
\text { action or strategy, in advance. }\end{array}$ \\
\hline Rehearsing & $\begin{array}{l}\text { To practice through making a speech, relating facts or anticipating } \\
\text { actions, in private. } \\
\text { Internalised preparation for an external exchange. }\end{array}$ \\
\hline Mulling Over & $\begin{array}{l}\text { To think about carefully; to consider a particular object or thought; to } \\
\text { allow the mind to wander or ponder over unspecified thoughts. }\end{array}$ \\
\hline Deciding & $\begin{array}{l}\text { Settling a question, uncertainty or dispute, leading to a final decision } \\
\text { on which to act. }\end{array}$ \\
\hline Reliving & $\begin{array}{l}\text { To experience something again, with or without associated emotion. } \\
\text { Derived from thinking about your own history. }\end{array}$ \\
\hline Prioritising & $\begin{array}{l}\text { To arrange, order and reject, organise or deal with something } \\
\text { according to its importance, urgency or other priority. }\end{array}$ \\
\hline Imagining & $\begin{array}{l}\text { To form a mental image of (something not actually present to the } \\
\text { senses). To suppose, think or conjecture/imagine. } \\
\text { Imagining extends possibility beyond existing boundaries. }\end{array}$ \\
\hline Clarifying & $\begin{array}{l}\text { Sorting out what you think about some issue, problem or person, } \\
\text { performed internally for and conditional on the subject's own } \\
\text { satisfaction. } \\
\text { To make (an idea, statement, etc.) clear or intelligible; to be free from } \\
\text { ambiguity, confusion or uncertainty. }\end{array}$ \\
\hline Budgeting & $\begin{array}{l}\text { Where you run through a conversation or discussion with people you } \\
\text { know or have heard of. }\end{array}$ \\
\hline $\begin{array}{l}\text { To plan or allocate the distribution of resources - in terms of money, } \\
\text { time or effort. }\end{array}$ \\
\hline
\end{tabular}

In the second and third interviews, I presented the participants with verbal descriptions of Archer's definition of internal conversations (inner dialogue, inner conversation, inner deliberation, internalised consideration, contemplation, to consider inside one's head (Archer, 2007, 2012)) and 10 mental activities (Table 5.2 and Appendix D). I then asked if these activities were familiar to them. Those who said the sort of mental activity was familiar, then explained how each activity worked for them and provided examples. Their examples included descriptions of the intensity and emotions of internal conversations (for example, confusion, frustration or enthusiasm) or their conditional use of mental activities. For example, Nan used mulling over and deciding only when looking for solutions to a community concern. The use of mental activities is an indicator of the presence of internal conversation and may also be evidenced in external conversation and writings. 
Table 5.3 presents the participant descriptions of the mental activities they believed they used.

Some cells have no response. I did not presume participants to have said no or yes, if they did not give an affirmative or negative response to a particular mental activity. For example, in interview two Elsie commented that she believed she only dwelt in the present. She connected only planning, clarifying and prioritising to her writing processes. However, in interview three, Elsie discussed her contemplation in rehearsing for conversations with her ex-partner. Otherwise, she made no response when directly questioned. Other participants appeared to have struggled with the concept or believed they had covered the point earlier. Occasionally, the interview conversation circled around as participants returned to elaborate on an activity presented earlier.

At times it was difficult for the participants to differentiate between Archer's mental activities. Some overlap was present in Kane's example of planning (Table 5.3), where he worked out in his head what he would say in a later conversation and the activity of rehearsing. When questioned about this, Kane explained that his planning was haphazard, unstructured thoughts, not associated with a particular event or person. The purpose of planning was to look at his own words and how he might phrase or rephrase his comments, whereas, he felt Archer's definition of rehearsal implied knowing what one was going to say, to whom and when. 
Table 5.3 Participants use of internal conversation and Archer's mental activities

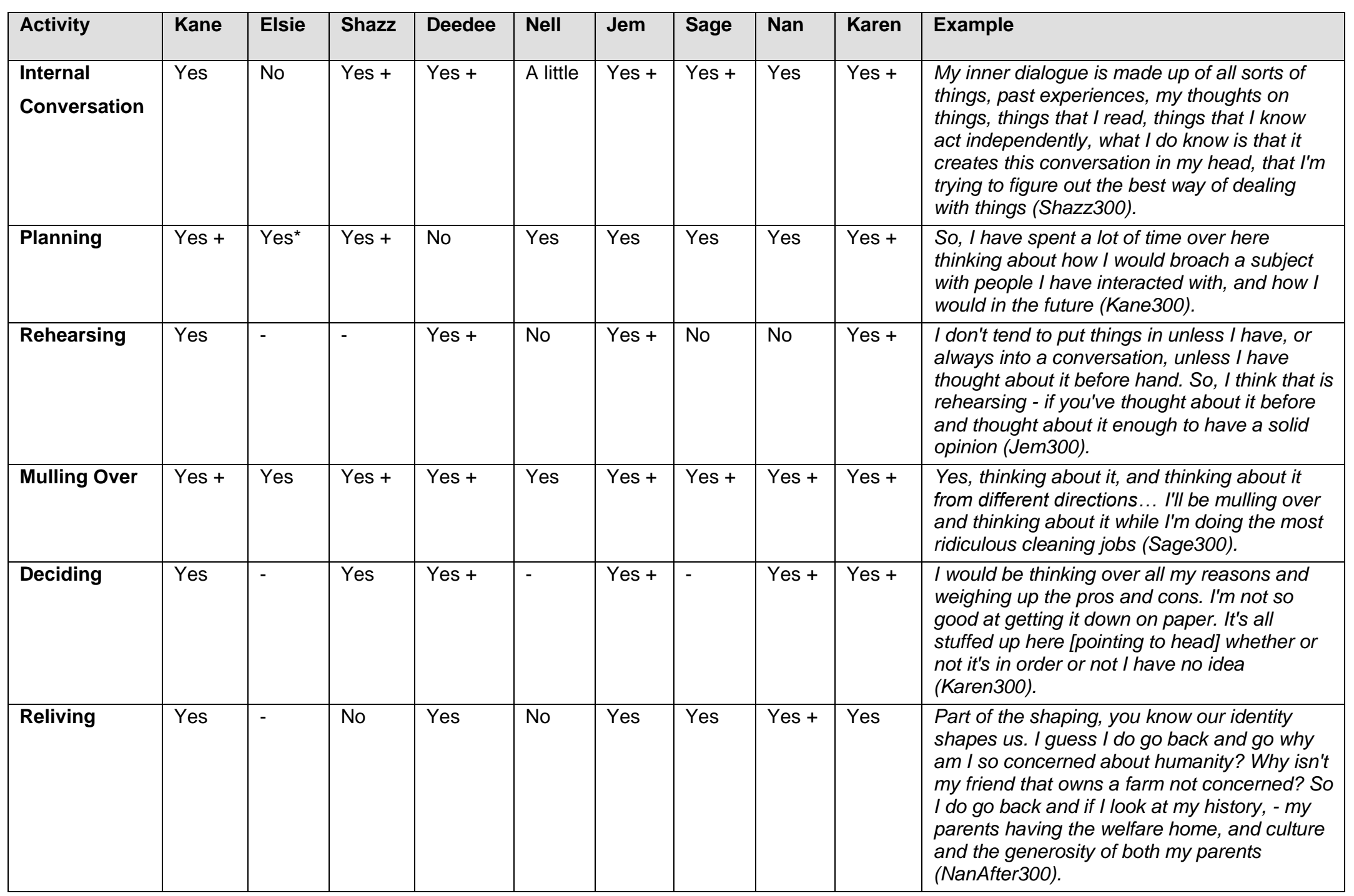




\begin{tabular}{|c|c|c|c|c|c|c|c|c|c|c|}
\hline Imagining & No & Yes & No & No & No & Yes + & Yes + & Yes + & Yes & $\begin{array}{l}\text { I'm definitely doing lots of ... and internalising } \\
\text { with how it might play out and changes and } \\
\text { trying to figure out what reactions might be in } \\
\text { response and how our situation is going to } \\
\text { play out (Jem300). }\end{array}$ \\
\hline Clarifying & Yes + & Yes & Yes + & Yes + & No & Yes & Yes + & Yes + & Yes + & $\begin{array}{l}\text { Because I'm quite aware that people think } \\
\text { quite differently. ... I've got some really great } \\
\text { friends that think really differently from me, so } \\
\text { I like to clarify. I like to think where they are } \\
\text { coming from, because I don't always think } \\
\text { that people think like me (Shazz300). }\end{array}$ \\
\hline Prioritising & Yes & Yes* & Yes + & Yes + & Yes & Yes + & Yes & No & Yes + & $\begin{array}{l}\text { To keep the relationship, I prioritise whether it } \\
\text { is worth fighting over something or bringing } \\
\text { something up. Because I never used to ... I } \\
\text { used to just speak my mind whether it was a } \\
\text { convenient time or not (Deedee300). }\end{array}$ \\
\hline $\begin{array}{l}\text { Imaginary } \\
\text { Conversation }\end{array}$ & Yes + & Yes + & Yes & No & No & Yes + & No & No & Yes & $\begin{array}{l}\text { If I'm trying to understand a personal situation } \\
\text { then yeah, I will have mental imagery and } \\
\text { imaginary conversations with people } \\
\text { (Elsie200). }\end{array}$ \\
\hline Budgeting & No & Yes & No & Yes + & Yes + & Yes & Yes + & Yes + & Yes + & $\begin{array}{l}\text { I spend a lot of time budgeting. I wouldn't call } \\
\text { it budgeting at all. I would call it juggling. I live } \\
\text { in the world where I have to juggle all the } \\
\text { time. There is never enough (Sage300). }\end{array}$ \\
\hline
\end{tabular}


The use of mental activities varied between participants. Jem affirmed that she employed all activities. She explained in detail how the activity was used. On the other hand, Nell identified with only a few activities and citied the advice of her counsellor to restrict her use of internalised thinking to help maintain stable mental health. All but one participant identified with the notion of an inner conversation or internal dialogue. Elsie, instead, referred to 'embodied conversation' a process of communication and mediation used within her intentional community. In interviews, the others described internal conversation in various ways, having found it "on-going and exhausting" (Shazz300), "internalises everything" (Deedee300), "constant" (Sage300) and "intrudes into all aspects of life" (Karen300).

All participants identified with the mental activity of mulling over. Mulling over was described as "letting something sit and filter out in body" (Elsie200), "considering a problem" (NanAfter300), looking at "different directions" (Sage300), considering "security and concerns for the future" (Deedee300), and something that "is natural to me" (Shazz300). The participants felt some of Archer's definitions overlapped, particularly imagining and imaginary conversations and deciding - clarifying - prioritising. However, Deedee made clear distinctions in prioritising relationships while clarifying understandings to decide which way she would act. In the next section, I present how students used the mental activities and their internal conversations.

\subsection{Internal conversations}

The regularity and intent of their internal conversations were analysed from participants' interview transcripts. This allowed me to consider the purpose, focus and nature of the use of the mental activities (Table 5.4). I differentiated between self-critique and consideration of other ideas to moderate and expand thinking, in the use of internal conversations. The regularity of participants' engagement with internal conversation and the level of intensity of those conversations were categorised into three frequency groups: high-on-goinginvolvement, episodic-for-a-purpose, and limited- or restrained-use. 
Table 5.4 Frequency, focus and use of internal conversation

\begin{tabular}{|l|l|l|l|l|}
\hline \multicolumn{5}{|l|}{ Internal Conversation } \\
\hline Frequency & Focus & Purpose & Nature & Participant \\
\hline $\begin{array}{l}\text { High-on- } \\
\text { going- } \\
\text { involvement }\end{array}$ & Others & $\begin{array}{l}\text { To mull over all views. } \\
\text { To consider possibilities. } \\
\text { To find solutions to improve the } \\
\text { lives of family and close friends. }\end{array}$ & $\begin{array}{l}\text { Emotionally intense. } \\
\text { Emotionally intense needing } \\
\text { management. }\end{array}$ & $\begin{array}{l}\text { Karen } \\
\text { Shazz } \\
\text { Sage }\end{array}$ \\
\hline $\begin{array}{l}\text { Episodic- } \\
\text { for-a- } \\
\text { purpose }\end{array}$ & Open & $\begin{array}{l}\text { To prepare for external } \\
\text { to others. } \\
\text { To consider theory. } \\
\text { close friends. }\end{array}$ & $\begin{array}{l}\text { Eogith family and } \\
\text { Origin of ideas important. } \\
\text { Concerned with not offending. }\end{array}$ & $\begin{array}{l}\text { Kane } \\
\text { Nan }\end{array}$ \\
\hline $\begin{array}{l}\text { Low-limited } \\
\text { or } \\
\text { restrained }\end{array}$ & Open & $\begin{array}{l}\text { In the moment consideration of } \\
\text { ideas. }\end{array}$ & $\begin{array}{l}\text { Directed towards the future. } \\
\text { Random thoughts. }\end{array}$ & $\begin{array}{l}\text { Nell } \\
\text { Elsie }\end{array}$ \\
\hline
\end{tabular}

\subsubsection{High-on-going-use of internal conversation}

Individuals in the high on-going use category were aware of sustained discourse within their heads. This deliberation took place either in direct words or thoughts which intruded into their processing of information, both as they were active throughout the day and as they rested at night. Five participants indicated they were aware of constant, on-going conversations inside their heads. All the views presented in the courses and by other people (family, close friends) were open to consideration in mulling over of ideas. In their daily activities Karen, Shazz, Deedee, Jem and Sage considered solutions and possible opportunities for action in situations they considered as either constraining or enabling. The focus of their internal conversations was on others - friends, family or social groups (for example, the poor or solo parents).

Three indicated the emotional intensity of these internal conversations could be overwhelming at times. Shazz found her internal conversation on-going, exhausting and stressful as stated in her interview: 
my inner dialogue is constantly checking and saying and looking at me through other people's eyes. I don't think it, but it has been helpful for me. But I don't think it's particularly healthy. I'd like to be able to not be considering other people all the time, when I speak (Shazz300).

Shazz held aspirational views of herself in all her roles - as a mother, daughter, partner, and member of two (her partner's and her own) extended immigrant families. Her inner dialogue constantly measured herself against other people's perceptions of those aspirations. Although Shazz claimed the reflexive thinking had "been helpful for me", her father had cautioned her about trying to do too much as she had "been burnt-out before" (Shazz300) by attempting to do too much. The mental activities of planning, mulling over, clarifying, and budgeting primarily featured in Shazz's constant inner dialogue. Shazz struggled with the possibilities for enacting citizenship projects against the realities of her life. Consequently, she saw a gap between her internal conversation of possibilities for improving her local community, the time and financial resources available to her, and her parenting and relationship priorities. Shazz could see an abundance of opportunities but was cautious about acting.

The constraints of existing socio-cultural, political or economic circumstances, of 'what was' and 'what could be' were mentioned by all in this group. Sage's internal conversation focused on the potential for improving the social conditions of her family, friends and social group (solo parents). She used the mental activities of planning, mulling over, reliving past conversations, and visually imagining, to be able to consider information from different directions. Discussing her thinking over the period of the citizenship courses, Sage said "I spent time imagining a better society with a different dominant story" (Sage300). She was mentally planning - budgeting resources and trying to clarify the intentions behind others' words. The courses provoked Sage, a mature learner, to scrutinise her personal situation with her internal conversation flow often interrupting her study tasks.

Mundane daily family-care activities provided a sense of solitude for Karen, where the complexities of family-work-study were worked through in her internal discussions. "Everything is a part of my internal conversation" (Karen300). It intruded into all aspects of her life, from hanging the washing on the clothesline - where she talked to herself out-loud, rehearsing discussions needed with family members - to prioritising, budgeting and 
organising family resources, activities and events. Internal conversation helped to keep Karen motivated about her own activities and goals.

Karen constantly mulled over external conversations to clarify what others meant and how this related to her own thinking. The citizenship course concept of reframing provided another level for elucidation. Karen was busy, fulfilled many roles and kept the emotions of her roles under control within her internal conversation. Despite attempts to keep their internal conversations and concerns for others contained within their own thoughts, Karen and Sage both found thoughts often slipped out. They found themselves speaking out-loud the concerns they had been mulling over.

In contrast, Jem and Deedee focused only on their own thoughts. Deedee used her internal conversations as a place for creative thinking which was not overwhelming. She was careful to limit its flow out into external conversation to maintain the relationships important to her. Jem's on-going internal conversation was restricted to working through what she considered logical arguments and considerations. This containment of internal considerations generated less emotion.

Jem valued her internal conversation as "everything is planned" (Jem300) at a high level of detail which helped her as a high achiever. She rehearsed her thinking through both internal and external conversations, especially in her discursive writings. Mulling over allowed her to work through big ideas, as she elaborated on in the interview:

It was there was in the back of my head, but I wasn't actively thinking about it. I was just letting it mull over and then when it popped up, I would let myself think about it. It wasn't like I wasn't letting myself think about it, it was just that I wasn't putting out at the forefront of my mind, and I found that while doing especially monotonous tasks sometimes my subconscious would work it out a bit better for me (Jem300).

Jem believed she needed to mull things over to have an opinion. Using her internal conversation also allowed her to budget time and mentally prioritise the areas she would work on.

Occasionally, there appeared to be a detachment between Jem's internal conversation and what she expressed externally, until she was confronted by others. "I do tend to be a little 
bit of an external person these days. I used to internalize everything... I can't switch off" (Jem200). In response to negative feedback from other students to her postings on the discussion forum, Jem was "learning to internalise more when externalising won't get you anywhere" (Jem200). She was slowly moderating between her internal conversation and her external conversation.

The main distinction between the two high on-going internal conversation subgroups was in the difference in focus. The three (Karen, Shazz and Sage) with strong community ties identified with their social roles as mothers and focused on the interest of others. The concerns of the others two (Jem and Deedee) were more self-centred.

\subsubsection{Episodic-for-a-purpose internal conversation}

Two participants used their internal conversations episodically (rather than all the time) to prepare for external conversations with whānau/family and close friends. Kane was concerned about presenting ideas and eventualities clearly, and not offending anyone. He distinguished between awareness at a subconscious level (where an idea might pop-up) and an in-depth conversation:

I have to define it in two ways - like in a conversation it is there in your subconscious and something that is happening in the background. And if I'm having a more in-depth conversation or reading or watching something or interacting with people, I do often think about what is going on without voicing anything... I, probably more than double or triple the words that actually come out in the interaction. In common normal interactions I will be mulling things over, I guess, on a pretty consistent basis (Kane300).

He planned his intended conversations, first, internally to avoid conflict with other people, while also trying to clarify the ideas mulling over in his mind. Kane had high empathy and concern for others' feelings and valued his relationships, so he used internal conversation to assist his actions.

I avoid conflict especially in discussions with people. Like you don't even raise the topic half the time. So, I have spent a lot of time over here thinking about how I would broach a subject with people I have interacted with, and how I would in the future ... 'cause I don't want to put them in a position where they are 
embarrassed or, or they embarrass themselves or I embarrass myself (Kane300).

Nan was concerned with empowering whānau and extended communities in considering possible solutions to social concerns. The emotion in her internal dialogues reflected her passion to bring about change. The idea of a constant on-going conversation was absent. However, Nan recognised her on-going internal conversation operated when she participated in external conversations and was processing ideas. In the interview, Nan stated:

I'm sort of, trying to work it out. I am processing because I'm working out what you're doing, for your research and I'm thinking, ok so now when we're wrapping up you've done the three, this is about the three papers, but and then thinking about how this is all coming together, you know these three papers [courses], and then how it all comes together, and the patterns and peoples thinking, and you know are they going to go out and do something to do with this or, I don't know I'm just, that's what I was processing (NanAfter300).

Nan mulled over ideas, looking for responses or solutions to problems, forming imagined projects. She relived previous experiences and spent time imagining "a new world", clarifying her understandings with people, and budgeting the resources she had available to act on issues or concerns. Nan was aspirational, thinking about the future with "dreams of a better place" (Nan100) environmentally and socially, and pursuing the solutions she had found in her internal conversation.

\subsubsection{Low or restrained use of internal conversation.}

Two participants limited their engagement in internal conversation for different reasons. Elsie saw internal conversation as counter to the "open, eye-heart sharing methods" used in her community. Instead, she believed her community's concept of embodied conversation allowed people "to respond instinctively, being present in the here and now" (Elsie200). Elsie saw citizenship as a reflexive concept, loaded with power and assumptions, fluid in its requirements of people and negotiated in space. As she worked by instinct, there was no need to clarify the understanding of others, rather she gave her attention to what was taking place in the moment. 
After her relationship break-up, like Kane and Deedee, Elsie used imaginary conversations on "highly personal stuff" (Elsie200) to prepare for external conversations. Although the embodied method she followed prioritised authenticity and collective intelligence over individual pursuits, Elsie's concerns for future employment beyond her community had entered into her internal thinking.

Nell, however, restrained her internal dialogue to control her anxiety and to maintain good mental health. Advised by counsellors to limit reflection and critical reflection, Nell acknowledged her mind peacefully drifted to mull over ideas when she was relaxed in places of solitude. Nell also described jotting down notes of her thoughts, connecting citizenship theory and ideas to her own experience. An example she used was her new understandings of ethics and how to act ethically.

In the final interview, Nell identified with Archer's mental activities of planning and mulling over, where "my mind dances around things" (Nell300), and prioritising as a means of budgeting. Planning for a purpose was a strong part of Nell's internal conversation, particularly how she would act to fulfil the requirements of social interactions. She used lists as a tool to deal with anxiety. Mental lists were ordered, locations and tasks prioritised to provide comfort and assurance - to limit discomfort. Internalised lists were examined each day and often externalised mid-conversation to her partner as "voicing it also helps like...kind of like comforts me" (Ne/l300). Upon completion of the list Nell could relax. Handling her depression and anxiety was more important to Nell than developing her reflexive thinking.

This section has covered the participants' explanations of the use of mental activities in their inner dialogue. The next section presents participants' use of external conversations of reflexivity distinguished by the type of discourse.

\subsection{Comparison with Archer's modes of reflexivity}

Archer $(2007,2012)$ identifies three dominant types of reflexive modes: communicative, autonomous and meta-reflexive. A fourth category - fractured reflexive - represents the underdeveloped reflexivity of younger or uneducated people, or interim confusion brought on by disruptive events in people's lives (Archer, 2012). At different stages in their reflexive development individuals are expected to display a dominant reflexive mode. Meta-reflexivity is at the pinnacle of Archer's modes and associated with higher levels of education. Thus, 
over a period of undergraduate studies an individual would be expected to progress from fractured or communicative reflexivity to autonomous reflexivity as they focused on their immediate goals, reaching meta-reflexivity as they planned for their future careers (Archer, 2012). Other researchers considered Archer's modes just as a starting or bridging point, rather than a finishing point, for considering people's reflexive interpretations of themselves, others and their own circumstances (Caetano, 2015a; Leibowitz, Garraway, \& Farmer, 2015; Nico \& Caetano, 2015).

Over the two-year period of this study only three of the nine participants' modes of reflexivity changed despite all completing their studies or a substantial part thereof. Despite the distribution of age and social and personal circumstances, at the start of my study metareflexivity was the dominant mode for all those with continuous internal conversation regardless of their study level (Table 5.5). In Table 5.5, the brackets around the second abbreviated reflexive mode indicates the mode was present but not as constant or dominant as the first mode. The use of two mode abbreviations separated by a ' $/$ ' indicates modes that appeared to be co-present at the time of the interview: for example, FR/MR modes were co-present for Deedee in interview one.

Table 5.5 Participant alignment with Archer's modes of reflexivity (2007).

\begin{tabular}{|l|c|c|c|}
\hline Participant & $\begin{array}{c}\text { Reflexive } \\
\text { mode at } \\
\text { interview 1 }\end{array}$ & $\begin{array}{c}\text { Reflexive } \\
\text { mode at } \\
\text { interview 2 }\end{array}$ & $\begin{array}{c}\text { Reflexive } \\
\text { mode at } \\
\text { interview 3 }\end{array}$ \\
\hline Kane & MR (CR) & MR (CR) & MR (CR) \\
\hline Elsie & AR/MR & MR (CR) & FR ${ }^{*}$ \\
\hline Shazz & FR/MR & FR & AR \\
\hline Deedee & FR & FR & CR \\
\hline Nell & AR & AR & AR \\
\hline Jem & MR & MR & MR \\
\hline Sage & MR & MR & MR \\
\hline Nan & MR/CR & MR & MR (CR) \\
\hline Karen
\end{tabular}

* Meta-reflexivity (MR), Autonomous reflexivity (AR), Communicative reflexivity (CR) and Fractured reflexivity $(\mathrm{FR})$

Communicative reflexivity was an additional mode for three of these people. Two people showed traits of autonomous reflexivity and a further two fractured reflexivity. Only three 
showed a shift in reflexive mode over the study period. These higher education students already exhibit high levels and stability in their reflexivity. As Bovill (2012) found in looking at the impact of mature students already engaged in work and study, it is natural for these students to consider their complex work-life situations in their internal conversations.

In the next five sections I describe how Archer's modes of reflexivity were exhibited by the participants in this study.

\subsubsection{Meta-reflexivity}

For individuals with meta-reflexive traits their internal reference points are values and the commitment people have to those values. Individuals demonstrating meta-reflexivity see the future as an unknown but all the possible actions they can think of are within their limits. They engage in critical thinking about their own internal conversations and the effectiveness of social actions. They ignore or challenge structural constraints and take advantage of enablements (Archer, 2007; 2012). To act on values is important in meta-reflexivity and to fail to act on one's values is disheartening. Developing and upholding their values is so important that there is no point at which their inner dialogue reaches a point of satisfaction (Archer, 2007). Participants in this study who appeared to act with meta-reflexivity have strong identities, confidence in their place and/or contexts and are community focused.

The first position was evident in the ways that Sage, Nan, Karen and Kane discussed citizenship and its rights and responsibilities.

- Sage's values were on improving the social conditions for her family and friends. In the first course she criticised the political influences that held people back. She stepped-out based on her new knowledge of citizen rights, hoping that encouraging people to vote would empower them and bring about social change.

- Karen's faith-based values were enacted in her attention to and care of those around her and her community. She volunteered and moved in many social circles and held plans to bring about change in her local society through her future career.

- Kane's community-centred upbringing permeated all aspects of his thinking, work and future plans, but he was uncomfortable that he was not upholding his environmental concerns sufficiently.

Nan's story demonstrated the strength of values and identity that characterise metareflexivity and its actions. Nan placed her mokopuna/grandchildren and their future and 
society's care for Aotearoa/New Zealand's environment at the top of her list of values. She considered a citizen to be someone who responded to the needs of society, was always on the lookout for creative and innovative solutions to problems and was reflective of the past. After participating in the final project, in interview three she said,

we now have the skills to practice that engagement, I think it was awesome. I loved how it all fell into place, and I liked how we can now take those skills that we've learned, out into the real world so we're going to know how to work with people, we're going to know how to ask for things when we want something, who do we network with, and they're all transferrable skills that we'll be able to use (Nan300).

She discussed herself as a responsible citizen who looked after the environment and upheld the Aotearoa/New Zealand identity of a society who cared for its people. Her motivation for her degree came from engaging in her community.

In the citizenship project, Nan led a successful environmental initiative. After completion of her studies and while applying for a public sector job, she researched, planned and applied for regional funding for a small rural community project she had been thinking about. Her internal conversations were the dreams, the imaginings she had of the future:

I'd like to see people not living on the streets. I'd like to see you know people out and about more enjoying themselves, or to go to the movies and all of the things that a lot of poor people have missed out on, and even actually a lot of working people. So, just that feel good about who we are as a country. So, I'm really big on our national identity, which is bicultural, so you know l'd like to see that grow even stronger and not change and go. ... You know we make our own decisions as a people, rather than go by what other people [say], ... we're helping people to create ideas and you know work for themselves (Nan200).

Nan's on-going reflexivity and focus on projects, which allowed her to build on her values, was typical of these participants.

However, Sage did not have the on-going meta-reflexive strength described by Karen, Kane and Nan. The socio-economic constraints of Sage's position held her back from engaging in further projects and the actions considered in her internal deliberations. She still retained 
the characteristics of meta-reflexivity but was unable to pursue her values at the level she dreamed of.

\subsubsection{Autonomous reflexivity}

Individuals with autonomous reflexive traits engage in self-contained internal conversations that build their confidence, motivation and self-reliance (Archer, 2007, 2012). They plan out and adopt strategies to deal with enablements and constraints to address their own concerns and priorities. As suggested by the title they are self-focused and self-referential, taking responsibility for their own actions. They readily consult with experts or look for independent information if required. They are risk-takers. Archer saw this mode as a stage in shifting social class in response to a growing understanding of the world (Archer, 2007). I believe Deedee, Jem and Elsie showed strong characteristics of this reflexivity mode under different circumstances. Jem and Deedee came from broken natal backgrounds and have had to be resilient and resourceful to make their own way through life. After her family split

up and one parent's health deteriorated, Jem cared for her teen-aged siblings to keep the family together. As she rationalised in interview two, she still felt responsible for their wellbeing.

I had a really big part, I guess, in bringing [them] up so, so for me especially especially being one of [their] caregivers - it is very easy for me to go to that place, because I don't always see [them] as a person. I see him as my baby [sibling]. And it's so different from seeing [them] just as a person (Jem200).

From an early age, Deedee moved between the state care system and her solo parent's neglect, eventually setting out to carve her own path as teenager. Deedee wrote in explaining her identity:

A personal example of [identity] fluidity is; that as welfare child of an alcoholic single [parent] of three, my home environment went very much against what was considered socially normal. Hence as a child, my sense of self was shaped by various negative experiences of exclusion, consequences of my class thread. However, with time, changes to my location, and with my role no longer being that of a powerless child, my sense of self is no longer controlled by that particular experience of home (DeedeeWW). 
There was an underlying strength of character and determination in Deedee's approach to life which had helped her to successfully carve out a career managing businesses and working with the public.

Both Deedee and Jem had developed strong self-confidence despite the lack of parental or state support at times. Jem described her privileged early life, providing access to good schools and activities, which built self-confidence and strength of character. At the completion of the third course, Jem was self-assured and self-focused in her view, and clear in her role as a citizen:

I feel more confident about responding to those things and being involved in those things. And not even just projects that I've started myself, but seeing things like that where they are asking, like asking for your opinion, and before even with that knowledge I didn't feel like I, I don't know. I don't know if I had the right to say things, but now I kind of understand it. I have a right to say how my country is structured especially when things are being over-looked, and I have a right to engage and trying to fix this. Fix it and try to participate in my country and in my community and in my area to try and improve those things, if I feel that's necessary. That's not just my right it's my responsibility really. And that's something that I didn't really perceive before the paper (Jem300).

In the past, both Jem and Deedee had needed to find and activate the resources to find solutions to problems. Jem spent a period of time homeless while holding down employment. The goal of her course of study was to upskill to become an advocate for those who do not have representation in society, for example, the homeless or other low socio-economic groups.

While Jem's circumstances and life direction had settled down, Deedee was again going through a period of change and instability. Deedee lived overseas in a culture where she had limited ability to communicate in the local language, no rights as a foreigner, no employment due to the lack of a university degree, and no income. She was financially reliant on her partner (who was also struggling to reintegrate into his natal culture), and emotionally reliant on him to help her communicate. She valued her national identity and the political rights she still retained even though out of the country. To vote in the 2017 Aotearoa/New Zealand elections she 
made the 6-hour journey up to the embassy to vote and turned around and came back and my family thinks that I'm crazy... so they thought I was absolutely crazy to travel one day to vote and turn around and come home the next day (Deedee200).

However, as new cultural and political limitations on her freedom were revealed, Deedee needed to keep her wits about her. She became a "master[s] of contingency" (Archer, 2007, p. 286). With no maternal rights to take her child out of the country, she needed to formulate a strategy for her family's future. To do so, she identified and worked on her language ability and status concerns. She identified the current political and social structures, enablements and constraints on her actions. As a part of her strategy to understand her options for action, Deedee used her social resources to gather more information and consulted expatriates in her local social networks (and on Facebook) who had more knowledge than she did. She asked questions on how they had dealt with their personal situations. She acted strategically.

To prepare for action, Deedee conducted long internal conversations. In these, she worked through the language constructs she needed, to make herself understood in undertaking daily tasks. Aware of the possibility for volatile situations to arise from misunderstandings about her concerns, she prepared for conversations with her partner. Her studies on citizenship had helped to build her inner reserves through developing her understanding her own identity and lack of ontological security (see Chapter 5.1).

Like Deedee, Elsie also fitted the definition of the autonomous reflexivity mode. Elsie's lifestyle choices were based on her values. She lived in a remote rural community group intentionally set up to pursue the environmental and life-style goals. As explained in interview one, Elsie considered the group "counter-culture" to outside society.

It's a very rich way of living, it's great. ... in terms of connections - living with streams, rivers and what not, and rich in terms of experiences of connections and togetherness. We do a lot of working on the land. For me it's very rich because it is close to the earth, close to the land and we have a lot of people coming in to learn how to grow food or live together; something people are very curious about. Meaningful, for me, a meaningful way to live (Elsie100).

As a long-term member of the group, Elsie had the status and the skills useful to practicing their alternative lifestyle. Her focus was on upholding her community ethos. "Living with 
people with similar values and objectives shapes our community identity and behaviour" (Elsie100).

Despite living within this tight-knit community where activity was by consensus agreements, Elsie displayed high confidence in her own ideas and analysis of external culture. She thrived on exploring academic ideas and looking at alternative thinking. She considered herself as independent and an "idealist person with good ideas" (Elsie200).

My sense of myself as a person that I can work with, that likes to work with ideas and in the world of ideas - reading, writing, conceptualizing, integrating and particularly thinking about taking that and pulling together, I have got a lot of skills in that area (Elsie300).

She had a clear opinion that the outside world did not hold the solution to the problems she sought. In the past Elsie had

been involved in lots of political movements for change and felt like they have made no difference but [studying the past political actions of citizens] makes sense that political movements happen, and change is over time. You might not see anything immediate, but if you look back over a span of time there's been shifts, and that shift has helped change something over time. And that was quite inspiring as someone who's thought - Ahh, this is not working. So, it is working. It's just a slow process and you have to, I guess, just keep on chipping away if you want to see things change (Elsie100).

Over the time of her study, Elsie had minimal engagement with the discussion forums or her student group work. She preferred to consult the articles of experts who she considered were useful to building her understanding and providing the tools she could use:

I think I would use some of those ideas about framing and story and how to connect to people emotionally through, to tell stories, to bring core messages through and not just using facts and figures and logic, but kind of using that deeper connection (Elsie300).

Developing an understanding of the concept of framing (introduced in the 300-level course) allowed Elsie to position herself both objectively and subjectively in evaluating her situation. She used this new tool to understand what occurred in her community meetings and 
people's presentations of their ideas. When her relationship ended, she recognised an attitude shift in community members towards her as a solo parent. She became more outward, focused strategically on looking for ways to become financially independent while retaining linkages to her community's aspirations.

All three autonomous reflexive participants' main focus was on their individual positions and maintaining the circumstances which helped them to achieve their goals. They had a strong sense of identity, confidence in their place and context, and prioritised their own concerns.

\subsubsection{Communicative reflexivity}

Communicative reflexivity is distinguished in individuals spending more time in 'thought and talk' processes, seeking others' ideas to develop or to confirm their own thinking than in internalised deliberations (Archer, 2007, 2012). This mode is a feature of reflexivity exhibited by most individuals at some point in their lives, but the dominant mode in younger people. The goal of this process is to reinforce or refine their own concerns, staying with the familiar rather than moving towards change or action. It is dependent on the presence of a trusted dialogical partner. In Archer's $(2007,2012)$ studies, people in this group used their inner conversation for short-term planning, talking first and deciding after consulting. They did not rely on longer mulling over or clarification of others' ideas. They sought confirmation of their place within their social class, wanting to stay within their social group (Archer, 2007). All the participants in my study demonstrated some strong characteristics of the mode of communicative reflexivity, with some showing more concern about staying within a social group. Four participants demonstrated strong resonance with communicative reflexivity.

By the third interview, Nell had made significant changes in her life - she had shifted cities and was in a committed relationship. While she did not see herself as politically focused, Nell had stepped out to join a political group. The focus of her attention was now outward looking, as she sought to secure places where her new views on citizenship could be tested and discussed. She was cautious looking for conversational partners she can trust, as she was uncertain that her views were acceptable to others. As she discussed in the final interview having changed cities and joined a political party:

I don't think I've changed too much. Oh, I've always been not very politically focused, but passionate about some politics. You know I will yell and voice my opinion. I will make people vote. I will debate with people on topics. I'm very passionate about it and I have started doing some volunteer work for a political 
party. ... But then I can't see the other side of it - like how it all affects money and everything else. I can only see one side, so it is hard to kind of think - is this selfish? Then am I trying to spread the word about it or how is it going to be enough to affect everyone? Or a majority? Or is it just going to benefit of small amount of people? There's like a lot of questions and analysing things a bit more rather than, yes, the student loan living costs should go up it's a good idea. It's more, well, maybe that might help people who have children on it (Nell300).

Nell was doing what Archer identifies as common to communicative reflexivity in staying put, securing her place in a group of similars; her activities and actions were focused around her own interests and concerns. She did not engage in the mental activities of rehearsing, mulling over, reliving, deciding and clarifying. She had connected with like-minded others on social media platforms and joined a social group. She had volunteered for a political party in her new local community where "it's like it is its own community, everyone has similar thinking" (Nell300). Nell had become a politically aware citizen interested in other global issues and action, and she wanted to confirm her ideas with others like herself.

Karen, Shazz and Kane also exhibited some characteristics of communicative reflexivity. They had links to familiar networks. These networks provided the base from which they worked out, searching out others' views in order to generate new thinking rather than just confirming their own views. Karen did not share her ideas on the discussion forums, instead she talked to elderly people in her church, the parents seated next to her at children's activities, other volunteers in her community and the young people attending the youth group she led. She explained in the first interview:

Talking to school community and teachers in the staffroom, it's always good to get different people's viewpoints on stuff. And I went out to some people and said hey if we had a really big earthquake who do you think is going to come and save you? (Karen 100).

Initially, Shazz discussed the citizenship threads with her close-knit family and friends. The topics had stirred up her thinking, but rather than clarifying and confirming her ideas, these discussions added to the turmoil of her internal conversations. She wanted to affirm her place in her extended family but was trying to reconcile the two worlds of her intellectual reality and social worlds. In interview three, she was fearful of moving beyond secure boundaries. 
[M]y inner dialogue is made up of all sorts of things - past experiences, my thoughts on things, things that I read, things that I know act on independently. What I do know is that it creates this conversation in my head that I'm trying to figure out the best way of dealing with things. So, I guess instead of just that primitive anger I'm having all these reasonable thoughts - like trying to see it from the other person's point of view. Trying to see it from an academic view, trying to see it from another. So, there's all these conversations going on and it makes me a bit stuck sometimes, because there is almost so much conversation that I'm going - so what? I don't know the best way to do it. So instead of just feeling the feeling and letting that out, I continue with this inner dialogue that doesn't actually. It does eventually get me somewhere, but it kinda makes me wish that I could have no inner dialogue to start with and I could just be angry (Shazz 300).

Meanwhile, Kane leaned heavily on discussion of citizenship concepts with his sisters, who he respected, trusted and admired. Their common upbringing was a secure platform. He also discussed the course ideas with selected work mates and his new friends while overseas. But Kane mentally planned for these conversations. He wanted to discuss and find out people's views but without causing offense:

I avoid conflict especially in discussions with people. Like you don't even raise the topic half the time. So, I have spent a lot of time over here thinking about how I would broach a subject with people I have interacted with, and how I would in the future (Kane300).

And so, he planned, imagining how the conversation might go:

I don't go through a rehearsed set of actions or discussion in my head directly as preparation to engage in a conversation, but I would say that I do them as part of my thinking processes. Basically, a discussion thinking of how a discussion might roll out, ... [Not] I'm going to meet this person in 10 minutes, this is how I'm going to go about the conversation, this is what they will say, this is what I will say in response. Not like that ok. More the conversation will happen in my head though and possible responses as part of the way that I think of it, I guess, and try to work out what I'm thinking about (Kane300). 
While these three showed some indications of engagement with their social groups to gather ideas, they also ascribed strongly with other mental activities that are not associated with communicative reflexivity.

\subsubsection{Fractured reflexivity moves to communicative reflexivity}

People with the features of the fractured reflexivity mode are those who are not able to use their personal characteristics to generate projects and act on them. They are not able to draw on their own strengths or social enablements to select a way forward (Archer, 2003). Underlying this insecurity Archer found, are disruptive events or circumstances which overwhelm their choices and means to act. Time spent in internal conversation on their social circumstances only intensities their distress.

In the first two interviews, Nell appeared to have fractured reflexivity traits. She did not dwell on ideas from the course. She did not reflect on her identity or belonging, to manage her anxiety and depression. This lack of reflexive processing was based on the advice of counsellors. She recognised her sense of self and connections could be distorted dependent on her mental health.

Belonging is not limited to the household but can be in any other form of community...I have clinical depression. My sense of belonging is often skewed depending on my mood. A sense of belonging is necessary to aid me in understanding my identity. The sense of belonging relates closely to the community, or citizenship, and to participation (NellWW100).

She did not rehearse or imagine situations or conversations before they occurred or relived previous experiences. Instead, she was a passive agent uncertain of her identity. Ontological security was an issue for Nell, the result of her family break-up, early departure from home and on-going fragmented contact with family. This uncertainty had impacted on her mental health and consequently her reflexivity, however, over the research project she formed a stable relationship and moved to a new city. Where previously she had exhibited characteristics of a fractured reflexivity mode, Nell had a home, company and plans for the future. Within this stability she was able to extend her thinking, plan and join with a new social group, which she wanted to belong to. Her shift to communicate reflexive mode also reflected this settled period in her life. 


\subsubsection{Fractured reflexivity and meta-reflexivity}

Contextual uncertainty also acts as a major constraint on reflexivity through an insecure identity. While Archer (2007) ascribes an insecure identity to young people, and attributes it to disruptive events and broken homes (which reflected Deedee's earlier background) it was not the cause of Deedee's current initial confusion and fractured reflexivity. It was the newness and uncertainty of her identity and role within her new environment, and as a distance student at university, that formed Deedee's struggle to make sense of everything and to plan ahead. Deedee's initial situation was similar to what Hung and Appleton (2016) found in their study of young people in-care moving out into society on their own. These shifts are destabilising as known reference points are removed or must be reimagined, which take time. However, unlike Hung and Appleton's (2016) participants who had limited internal conversation, Deedee's internal conversation was on-going. Deedee became more selfcontained and controlled in her reflexive thinking as she settled into her new country and her motherhood role. Over time, she regained a sense of identity and purpose and accepted her role in her community as a mother and an outsider. This allowed her to focus on her priorities and concerns particularly in identifying the constraints of her situation. Over the period of the study she moved on to demonstrate autonomous reflexive attributes.

In this section, I have presented an analysis of findings on the reflexivity modes used by higher education students in this study as they considered concepts and values of citizenship. In contrast to Archer's participants tending to exhibit just one dominant reflexivity mode, I found many participants showed characteristics of dual modes as they considered their citizenship studies and moved between the facets of their work, study and home lives. Changes in their personal and social contexts had a marked impact on their opportunity for thinking and acting on their citizenship values as personal values reflected their priorities. In the next section, I look at how external consideration was used in conjunction with internal deliberation. 


\subsection{External Conversations}

Both oral conversation and writing are used in different ways to help reflexive thinking. Archer's internal conversation and reflexivity modes refer to external conversation as having a subordinate place in deliberation. However, other researchers suggest people use a variety of mechanisms to gather or share ideas, to test the logic of their thinking or to organise ideas (Caetano, 2017a, 2019; Chalari, 2007). External conversations are believed to enrich internal deliberation. Therefore, I analysed the forms of social interaction exchanges my interviewees participated in.

Verbal external conversations were also subject to regularity, and the nature and conditions of use described in Chapter 5.3. Again, I use regularity here as a qualitative observation of how participants felt they engaged in external discourse. I also describe the level of intensity of their conversations. The purposes of the external conversations are grouped into three areas (Table 5.6). First, to gather or understand other ideas, to clarify others' views, or to critique by looking at the framing of ideas. Second, a place to process one's ideas with or without consideration of others' viewpoints. Third, a place to try out the logic of their thinking or to disseminate their own views. Two groupings, similar to the internal conversation groupings, have been identified: Highon-going-involvement, and Episodic-for-a-purpose.

Table 5.6 Frequency, focus and use of oral external conversation

\begin{tabular}{|l|l|l|l|l|}
\hline \multicolumn{5}{|c|}{ External Conversation: Oral } \\
\hline Regularity & Focus & Purpose & Nature & Person* \\
\hline $\begin{array}{l}\text { High-on- } \\
\text { going- } \\
\text { involvement }\end{array}$ & Community & $\begin{array}{l}\text { To draw on others' expertise. } \\
\text { To gather ideas. } \\
\text { To mull over all views. } \\
\text { To compare or consider } \\
\text { possibilities. }\end{array}$ & Reserves self to hear others & $\begin{array}{l}\text { Karen } \\
\text { Shazz } \\
\text { Can be emotional }\end{array}$ \\
\cline { 2 - 6 } & Individual & $\begin{array}{l}\text { To compare own ideas } \\
\text { To disseminate own views }\end{array}$ & $\begin{array}{l}\text { Own reality impinges on } \\
\text { conversation }\end{array}$ & $\begin{array}{l}\text { Deedee } \\
\text { Jem }\end{array}$ \\
\hline $\begin{array}{l}\text { Episodic-for } \\
\text {-a-purpose }\end{array}$ & Purpose & $\begin{array}{l}\text { To give intuitive whole person } \\
\text { responses. } \\
\text { Managed to gather ideas. }\end{array}$ & $\begin{array}{l}\text { Functional } \\
\text { Socially constrained }\end{array}$ & $\begin{array}{l}\text { Elsie } \\
\text { Nell }\end{array}$ \\
\hline $\begin{array}{l}\text { * Nan, Sage and Elsie did not identify as using external conversations as a part of their reflexive } \\
\text { processes but I found evidence of Elsie doing show in the data }\end{array}$ \\
\hline
\end{tabular}




\subsubsection{High-on-going-involvement}

In this grouping, individuals looked for opportunities to discuss ideas with other people throughout their daily routines. The majority of participants took part in open discussions with others, but the content focus of these discussions on communal or individual ideas distinguished two subgroups. The first, the community focused subgroup, sought to draw on others' interests, experiences and understandings to inform their own thinking. Other people are a resource that may be tapped into. For example, Karen was involved in social ventures on an on-going basis through her family activities and her voluntary work. She discussed citizenship course ideas with fellow workers, church members and her work colleagues. She then tried to incorporate all these views into her considerations of future actions.

Shazz's external communication was also important for her, particularly in relation to her sense of belonging in her family groups and understanding of ideas. She explained in the first interview:

I discussed it [citizenship] a lot with a lot of people, with people from all sorts of different parts of my life. ... I would just get really passionate about things or it brought up things that I was really interested in, and so I would talk to different people about it. My Mum - I talked to her a lot about it - I talk to her a lot anyway, but it was good to have some discussions with her about it. My Dad, and my friends who have jobs in public policy, marketing, and things like that. And I felt like I suddenly had some stuff. I mean I was really interested so I had some questions to ask them, to find out what they were doing and suddenly their stuff was a whole lot more interesting (Shazz100).

Shazz described herself as a talker and wanting to have a voice to bring about change. Yet she did not feel she had anything to offer in conversations. The course readings had given her ideas to consider and she could then discuss these with her parents and friends to question ideas and process her own thoughts. "I just think the more you can understand about how people think, because they do things really differently, and how different they work" (Shazz300).

Kane similarly recognised conversations with others as a way to work out what he and others think. In the first interview, for example, he used the research interview with me to elaborate on his own ideas, to think in the moment, and to talk aloud the about ideas of citizenship he found challenging.

I saw some similarity to my circumstances of being a first-generation Kiwi and associating so strongly with NZ and everything because of it. l've just been thinking 
of ... but perhaps the view I haven't thought of so much or the other view ... Guess I've always just lumped myself in with them, ... I hadn't really considered that much in the past ... and I guess that that was something that popped up [now] that I thought was quite interesting. [It's] pretty obvious, but we don't think about it that often (Kane100).

Participants used various social interactions to broaden their own understandings and to compare what others had experienced against their own life experiences and understandings. In doing so, they held back their own thoughts to first listen to others, sometimes seeking out people beyond their immediate confidants to draw more widely on others' experiences. While listening they sometimes shared their own positions and thinking to gauge another's response to their ideas. The emotions triggered in these conversations could be fully embraced and shared or held incheck. Kane's thoughtfulness in preparing for discussions was because he admired and valued the honesty of his siblings and wanted to hear what they had to say.

The second 'high-on-going-involvement' subgroup also compared their thinking with others, but more to endorse or confirm their own position. In her internal conversation, Deedee prepared for external conversations, so she felt more confident crossing language barriers to ensure she can get the resources she needed from people. Deedee had a strong internal deliberation and externally made lists, planned and prioritised what actions she needed to take in response to challenges as they arise. She also rehearsed every conversation within the privacy of her mind to battle the challenges of language difference in her adopted country. She continuously mulled over feelings of insecurity and concerns for the future arising from her family's mixed citizenship and rights. In carefully controlled conversations, she consulted with other expatriates on their citizenship concerns and how they have managed their situations. She considered all the possibilities that she might act on, and the impact of particular stances, before externalising with her partner, justifying to me:

I won't bring anything up until I've kind of thought about every single possible option, probably should have been a lawyer. You know every possible outcome. And if I can't figure it right out then I don't usually bring it up. But not all the time. No, I think that I mostly, I mostly keep things to myself (Deedee300). 
Deedee pushed emotions aside as pragmatism prevailed in finding a comfortable path to negotiate difficult subjects in cultural or personal relations (for example, discussions with family members on culturally different gender roles and their expectations).

Jem used external conversation to disseminate her views to enlighten others. She saw herself as the only one prepared to stand up against popular opinion:

Everybody shoots the messenger, but I only highlight my perception on a subject that already exists. I don't 'create problems' I just remind us all that they haven't gone away. I create shame, guilt, anger and even resentment (Jem300).

In contrast, Jem used external conversation regularly as a place to assert her own ideas regardless of the responses. She believed having academic knowledge or university experience validates her thinking as 'the' informed understanding. This sanctioned any emotions Jem's words invoked in others through what she said.

\subsubsection{Episodic-for-a-purpose}

This episodic-for-a-purpose group used their external conversations with specific intent, when they were ready to do so. Its characteristics differentiate it from the previous group by the conversation being restrained, functional, or even constrained.

Elsie's community context and its emphasis on 'eye-heart-meaningful' conversation means casual or spontaneous conversations to discuss issues external to the community were not prioritised. Conversations to engage in discussions might be initiated by those outside the community but 'full sharing' may not occur with those people. Elsie believed a person moved between social interactions in an "intuitive" manner, committing completely to the current context rather than following on-going internal or external discussions. "I've tried to be present with what I'm doing rather than going over into different spaces" (Elsie200).

Nell managed her external conversations (as she did her internal conversations) to limit her anxiety and social interaction pressures. She was selective in activities and outings which removed her from her home context, but open to new social exchanges within the security of her home. Maintaining a good state of mental health made quality conversations a priority that could be planned for and managed to gather others' ideas, including social media conversations. 
This section completes the findings on oral external discussion as a resource, or means, of reflexive processing. Most of the students in my study used this form of external conversation as a means to complement the resources provided in their course, to extend the knowledge available to them. In the next three sections, I consider how participants used written external conversation in course assessments, on discussion forums and social media as a part of their reflexive processes.

\subsection{Course assessment as written external conversation}

The process of putting thoughts into writing generates new ideas or forces the writer to revisit their current position. In considering or explaining their own thoughts, previous conversations are reconsidered, and their content informs current thinking. In this way, writing as external conversation can be creative or used purposefully - to process or test relationships between ideas, to organise ideas such as making lists, which may or may not be followed (Caetano, 2017b). More formal writing like course assessment is targeted towards an audience and purpose.

In the citizenship courses, participants had to prepare written documents as course assessment. The writing of these were purposeful responses to assessment requirements and the teacher as the audience but may contain elements of creativity. Writing creatively was used to generate a schema to examine thinking, through the processes of planning, summarising, and the consideration of evidence. Elsie used writing processes as a part of her reflexive processes in preparing, planning out and summarising ideas and her thinking, to present her final argument. She believed her internal understanding was developed in writing as she allowed ideas to "sit and filter out of the body" (Elsie 200), prioritising ideas for writing. As demonstrated in the 100-level assignment, Elsie focused on big ideas and related them to her own experience:

Citizenship is usually associated with sanctioned membership of a country (Kahu, 2015). For example, I am a New Zealand citizen, and this comes with responsibilities such as paying taxes and obeying laws, as well as privileges such as paid healthcare while pregnant. ... However, my community citizenship can conflict with my identity as an 'everyday' New Zealander because we have developed a strong internal 'counter' culture reflecting an absence of common national heritage and a more communal approach to wealth and land management. This illustrates that identity threads, even around something as broad as citizenship, can contradict (ElsieWW100). 
Discursive writing acted as the catalyst for Elsie's reflection, acknowledging realities and considering future possibilities. Deedee also used writing to process and organise ideas - as a means to compare her experiences with course ideas and to present her viewpoint. In addition, she looked at assessment feedback as a means of affirming her place as a student in academia. She then had the confidence to use the understandings she had reasoned out (in earlier critical thinking and assessments) to present them on social media platforms and discussion forums.

\subsection{Discussion forums as written external conversation}

Generally, online course discussion forums provided an opportunity for students to create a learning community of peers. The asynchronous nature of discussion forums provided more regular opportunities for student discussion and feedback than assessment work, as the frequency and nature of engagement could be fitted within personal schedules (Kahu et al., 2014; Poskitt et al., 2011). This distance learning community may be the only contact students had with their peers and teachers. However, use of the discussion forums also required time. Nan, Shazz, Sage and Karen made postings to the forums. Nan read and observed what was being written (the topics, tones and responses) and reflected on the content. At a later point she connected seemingly disparate ideas in drawing to the conclusion that a sense of loss of place was the cause for other contributors posting racist comments.

Deedee who felt the isolation of physical, temporal and cultural distance regularly posted questions, comments or discussion to the forum. Over the 12-week period of the 100-level course she made more than 50 posts. She responded to other students' contributions and offered her own ideas for discussion. She compared her current cultural context and constraints with her experience of the Aotearoa/New Zealand context, as she worked to make sense of what citizenship meant to her. In the 200- and 300-level courses, there were fewer opportunities for discussion with her wider group of peers as group forums were used more to organise assessment tasks than to discuss ideas.

Kane and Jem were also regular contributors to the 100-level forum. Kane was more reserved but read posts and was encouraging in his responses to others' opinions. He was cautious in his wording and clearly framed ideas as his opinion or understanding of materials and was open to others' thoughts. In total, Kane made over 25 posts engaging in discussions. On the other hand, Jem used the forums as a means to disseminate her own views and perspective. She made more than 20 lengthy postings, several pages long, of personal opinions illustrated by her experiences 
in the first course. Jem used writing on the posts as an informal venting of ideas, as demonstrated in the following post:

It took a long time to undo the attitudes my dad had normalised for me. In my late teens, I went through the typical "I'm not racist but....." sentences (thinking myself enlightened but really still as racist as ever). It wasn't until I started taking university social science papers, in my early twenties that I really learnt about the depths of racism in New Zealand and within myself (JemDF100).

Jem's discussion forum dialogue was one-sided. Her position was that her study had helped her understand racism. Jem did not respond to or comment on others' responses to the discussion thread until she presented a meme - an image which she believed humorous which was challenged by others as 'racist'. Jem then felt the need to justify her actions, arguing the message was rushed and may not have been articulated as clearly as she was thinking. She saw this, and readers taking her comments personally, as the reasons for the reactions to her post. While she believed her intentions were to open up the dialogue on racism and to challenge public discussions of racism, she saw the course forum as an opportunity to present her own thoughts independently of the social element of the course. In the interview with me, Jem remained defiant on her position and that her role in the discussion was to illuminate others' thinking.

Shazz used the 100-level discussion forum as a way to be reflexive about topics, by looking at other respondents' comments. She noted tension in the postings and cautiously posted her own perspective, delighting in receiving a reply. As she explained in the interview:

There was only one where there was a bit of tension, ... I wanted to express my spiritual side. Cause I feel spiritually connected to places and sometimes l've been told that it wasn't my place to be spiritually connected... or I couldn't work out if it wasn't my place. My tutor wrote back something amazing to me [joy in voice] .... Even the forums, I loved. I loved the way people were brave enough to say what their truth was - even though it was unpopular (Shazz100).

Where Shazz used writing on the forum as a means to be reflective, Nell was more cautious. Writing down notes of what she wanted to say first allowed her to actively engage in wider conversations on selected social media platforms. In the 300-level course, Nell used the more intimate in-group software and chat opportunities provided for group work extensively. Less intimidated by the more personal format, she found the "open dialogue with the group" or external 
discussions on the course useful as "it's not like...thinking alone, where you might miss considering ideas from a particular angle" (Nell300). Nell wanted to confirm her own ideas and interests within a broader range of people's thinking. Overall, the course discussion fora and alternatives offered were participated in and used in quite different ways by these participants.

\subsection{Social media as written external conversation}

Some individuals regularly used social media platforms to follow political and personal interests, and as social networking systems. Users identified with the social groups they joined online or could use the platforms to gather specific information, presented by interest groups. Nan, Jem and Nell were regular contributors to social media forums, but for different purposes.

Nan used her voice in political polls and associated social forums to offer alternatives and solutions to the dominant voices she read, and to represent her community-based values. Nan's emphasis was on keeping her contribution consistent with her values of improving others' situations for social change. She used social media to make representations for those who could not stand up for themselves "lifting people from the bottom up" (Nan200) and to ensure a balance of perspectives was presented. Jem also felt she was representing those without a voice in her use of Facebook and other community forums to present her own thinking. She considered "everything I write is reflective and reflexive" (Jem300) as she had an awareness of her own cultural beliefs and assumptions. Informed by her past experiences and current studies Jem considered herself an authoritative voice in balancing discussions. However, Nell was less confident in presenting her own voice on social media. It was only after working together with likeminded people in the 300-level group project that she felt more comfortable to specifically advocate for understanding of mental health conditions of anxiety and depression on public forums. The key uses of social media platforms by these participants were to offer alternatives to the prevalent voices on forums, to present their own opinion, or to advocate for a particular interest group. Social media provided a venue for both gathering ideas and voicing concerns. 


\subsection{Chapter summary}

I have outlined the main findings on internal and external conversation and participants' reflexivity in this chapter, using examples from the nine individuals' stories gathered over time.

Internal conversation was a part of all these participants' lives and was used broadly to compare and contrast ideas presented in their studies with their daily life. Using internal conversation in a more focused manner allowed these individuals to examine particular citizenship ideas, or to allow ideas to float or mull around without predetermination. Intense and focused inner deliberation allowed for the examination of specific ideas and for participants to critique their own or others' ideas. Of particular focus were those ideas which were confronting, or when the participant wanted to prepare for external conversations. These inner spaces served as places of moderation, or to expand on an individual's thinking, in particular in consideration of identity and place in society.

Archer's modes of reflexivity do provide a platform for further exploration of university students' reflexive processing of value-laden ideas presented in this study. This diverse group of participants did not fit with Archer's theory of a reflexive progression (from communicate reflexivity, through autonomous reflexivity to meta-reflexivity) by age and education. However, disruptive events and uncertainty about one's identity and belonging does appears to produce fractured reflexivity, which may be temporary.

Characteristics of concurrent modes of reflexivity were present in some participants' reflexive thinking. Changes in modes of reflexivity were found and appear to depend on changes in an individual's environment, disruptive events or variations in their circumstances. Temporary changes in context and events affecting their family affected several participants priorities for study and had an impact on their reflexive processes.

In external oral discussion, the participants viewed other people as a source of information and perspectives; their thinking was valued. Ideas gathered from immediate family and friends, of from strangers encountered in everyday life or on social media, contributed to widen their understandings of social concerns. These new perspectives could be examined in the solitude of participants' inner conversation. External conversation was also a place for participants to process their own ideas and to test the logic of their thinking. A few 
participants used external conversations as platforms for disseminating their views to their peers or a wider audience.

Participants used written discourse in assessment writing or their own note-making in a manner similar to Caetano's 'creativity' (Caetano, 2017a) to clarify their understandings or to test new thinking. Some were very directed, purposefully responding to particular tasks and audience, rather than demonstrating the connection of academic ideas to their own lived experience. Others used writing as a means of processing and organising thinking. A few saw the process of writing as an opportunity to present their opinions meaningfully to represent groups they perceived as having no voice.

To be reflexive about citizenship and to act on one's inner deliberations requires an individual to have a good understanding of their identity, values, concerns and priorities. Drawing on knowledge of their fit within society and understanding their own and others' ontological security enabled individuals in my research to be more receptive to the diversity of people and circumstances that they encountered.

In the next chapter I discuss these findings in more detail and how they relate to the ongoing conversation on Archer's reflexivity theory and internal conversation methodology. 


\section{Chapter 6. Discussion}

\subsection{Introduction}

My qualitative longitudinal research project looked at the reflexive processes used by higher education students to consider value-laden ideas they encountered in their university courses. In order to answer this question, I asked students about their lived experiences of learning, their values and priorities, who they discussed their course ideas with, and how they went through the processes of weighing up the ideas introduced in the course or with their peer group. In this chapter, I discuss the findings and explain their contribution to current understanding of reflexivity and internal conversation theory and methodology.

First, I discuss the study contexts and the students' personal contexts which influenced their identities, values and reflexive experiences. I build on this information to consider how these students 'fit' Archer's modes of reflexivity $(2007,2012)$ including their ability to act on their new understandings and values of citizenship. I then present my emerging framework that seeks to explain the conditions that affect diverse higher education students' reflexive deliberations on their values and concerns in a set of courses intentionally designed to challenge their existing knowledge and assumptions. Reflexive processes involve the individuals deliberating through their internal conversations and external discourses on their values, concerns and life goals. They may rationalise, merge or discard concerns and modify priorities in order to reach a life position that is a temporal and "sustainable modus vivendi" (Archer, 2012, p.124), suitable for confronting the challenges of the globalised world. The framework is based on my synthesis of findings from the participants' experiences, comparing and contrasting their accounts, and with reference to the literature presented in Chapter 2.

One important finding introduced in Chapter 4, was that while some participants' attitudes remained resistant to the restriction on their choices, all participants were open to considering the ideas on citizenship offered in the courses and in their interactions with their peers. This meant in Chapter 5, I was able to examine more closely and describe the 
reflexive processes used by nine participants in considering citizenship and their personal circumstances over time. I found internal conversation was considered as a normal part of many of their lives. These students used their external conversations with others as a means to fill in the gaps in their knowledge, to check their understanding and to test the new ideas that evolved out of their reflexive thinking. However, those who lacked dialogic partners due to personal circumstances tended to use writing as a means of reflexive thinking of the ideas encountered. To engage in reflexive thinking, all these students required a clear sense of their identity and understanding of their concerns.

\section{Reporting on internal conversation}

Reflexivity theory places internal conversation as a first-person encounter. As such it is private and not accessible to others (Archer, 2003, 2007). However, it is made accessible to a researcher by examining an individual's narratives of their thinking processes and looking for particular reflexive characteristics or mental activities of thinking and deliberation on projects, values and concerns. While Archer focused solely on the internal conversation or deliberation, other studies (Caetano, 2017b; Chalari, 2007) have included narratives from external conversations and writings. By using segments of the participants' interviews, postings on discussion forums and written narratives, I also wanted to present a fuller picture of the whole. For while,

narratives are good for conveying life and colour, ... there is always a danger of losing the plot...[and] there is a large debt owing to the respondents to incorporate their own takes upon their own narratives (Archer, 2007, p. 269).

This discussion chapter takes a "distanced overview and attempts to synthesize the material present" (Archer, 2007, p. 269) back to other research, rather than relying solely on interviewees' dialogue.

The following discussion of reflexivity focuses on the following areas:

- How context and change in circumstances set the scene for reflexive processing

- How social interactions build resources which inform internal conversation

- The communal or individual foci of reflexivity

- The presence of dual, multiple or single modes of reflexivity, and

- How reflexivity enables but does not determine action and agency. 


\subsection{Reflexivity and contextual continuity and discontinuity}

To engage in reflexive processing an individual must be aware of their thinking about themselves and their context (Archer, 2007). Yet reflexive lives are not lived out in single contexts, but multiple, sometimes visibly intersecting arenas, or social networks (Dyke et al., 2012). The embedded case for this study was a set of three citizenship courses in an Aotearoa/New Zealand university. As students encounter the formal and informal structures of academia, they develop the affective, cognitive and behavioural characteristics of 'a student' to engage and achieve their goals (Kahu, 2013; Weidman et al., 2014). However, being 'a student' is just one of many of the identity roles and characteristics described by the participants in my study. Each characteristic of being a distant, online, mature, part-time or full-time student is another subset of identity and therefore influences both the nature of reflexive thought and the time spent in reflexive processing.

The distance learners in my study were not young, so-called 'traditional' undergraduate students who retained contextual continuity with family and childhood backgrounds while in the process of developing their independence, as described by Archer $(2007,2012)$ and Luckett and Luckett (2009). Nor were they surrounded by the tight social networks of old, traditional societies enclosed by national borders, that were undergoing dramatic or disruptive changes (studied by Caetano, 2015b, 2017a; Case, 2013; Mrozowicki, 2011). Instead, these distance students had diverse study-work-family-life networks and social and study commitments (see Chapter 4), and most were already contextually distanced from their natal contexts. They were older, some first-in-the-family university students, or students with gaps in educational experiences, study periods and knowledge of university study. These characteristics are typical of the distance study cohort at the contributing university (Kahu \& Gerrard, 2018). Many participants had spent time living in other cultures or countries. The diverse, 'non-traditional' participants in my study may be in different states of contextual continuity or discontinuity from that of their so-called 'traditional' student counterparts.

Contextual continuity is demonstrated in people who seek to remain within the stable social environments of their childhood (Archer, 2012). Such individuals manifest communicative reflexive traits as they engage in conversations with others from their natal contexts to reaffirm or check their views are consistent with their associates and their shared values. As discussed in Chapter 4, these non-traditional students studying in the citizenship courses already had a strong sense of identity reflecting their varied life experiences and roles. They 
no longer self-identified with their natal contexts. Separation from their childhood, family and local community contexts had already taken place once or many times. They chose the parts of their identity they wished to reveal (Oyserman, Elmore, \& Smith, 2012) to their study peers. Only one of the four participants who had demonstrated communicative reflexivity (CR) characteristics showed contextual continuity. Shazz still lived in her childhood community, close to her parents and relied on their input into her thinking and actions. On the other hand, Deedee and Nell, with their Fractured Reflexivity (FR) traits, provided evidence of contextual incongruity. They had values, lifestyles or other arrangements which clashed with those of their upbringing (Archer, 2012), but they also showed Communicative Reflexive (CR) traits in seeking groups with common values to which they could belong. However, there was a characteristic from their study context which cut across all 31 participants' situations. Using the technology available as distance students, they had had no need to move from their homes to attend classes. Therefore, being a university student was an additional or temporary role, set apart from their natal backgrounds and integrated with their other roles as workers, parents and members of communities.

Contextual discontinuity arises when people encounter the diversity of values and beliefs of new environments (Archer, 2003) and when their own beliefs do not reflect those of the people around them (Archer, 2007). Individuals then need to be constantly evaluating and arbitrating between these new ideas, ways of being, and their existing values and beliefs. In this, they consider what is important to them and the possibilities and courses of action. Having well-established beliefs and principles as adults did not stop participants in my study from encountering new ideas or different viewpoints; particularly new ideas on the impacts of current and historical events on their citizenship. Nan's realisation of the connection between her ties to whenua/the land and refugees' loss of rights to live in their own countries had a substantial impact on her thinking. For Māori as tangata whenua (indigenous to the land) connection to the land is paramount. In being challenged to think about the plight of refugees, Nan appears to have drawn back on her Māori culture in accepting people from other cultures because of their displacement from their homelands. Her action is consistent with Elder-Vass's (2007) claim that habitus in a person identifying with their social origins, needs to be considered in the structure-agency relationship. Similarly, the male student (Chapter 4.4.2.) who was contextually dislocated from his family upbringing and had established the connection to his extended family member's principled protest, was once again being pulled back into his wider family. Neither of these positions reflect social 
conditioning which Archer believed was the substitute for habitus (Archer, 2003). Instead their social origins are integral to their identity even though they are contextually distanced from their childhood environments. In both cases, contextual discontinuity was an operational position that allowed Nan and Billy to reconsider and reconnect their values to the influences of their natal cultures.

Technological advancements and movements of people as triggers of rapid social change were considered to contribute to the breakdown of national cultures and their inherent structures (Archer, 2007; Beck et al., 1994). As structures break down, individuals must become more self-contained and self-reliant. Archer's fears of the disintegration of the family as a structure may have been somewhat pessimistic. The widespread prevalence of technological advancements means person-to-person contact is no longer limited to specific places and times within traditional social structures, which Archer also believed were breaking down (Archer, 2000, 2003). Instead, all participants in this study used the technologies of video conferencing and social media as a means for extended contact with friends and families. In particular, they maintain more frequent contact across large distances and with greater ease. The participants also joined social interest groups and networks beyond those that they might have otherwise encountered. They used the resources of these extended contacts to inform their thinking, planning and decision making. This finding supports the argument that Archer's individualist concept of reflexivity places too much emphasis on social isolation as a consequence of change (Tsekeris \& Lydaki, 2011). It could also be argued that in following Giddens and Beck's reaction to change Archer, in turn, places too much emphasis on the constraints of structural change over individuals, rather than granting agents the ability to make their own decisions about how they adapt to change.

The diverse learners in this study appeared flexible and open to change. They were mobile, comfortable with moving between cities and countries, high users of technology in their social engagements and interested in understanding dissimilar viewpoints. Yet, when confronted with restrictions on their university subject choices (see Chapter 4.3) the attitudes of some of rejection and resistance indicated they were not all as open to changes in their personal circumstances. The question then is: did this affect their agency? To be agentic there needs to be some form of compatibility or incompatibility between structures and a proposed project and hence an agent's response (Archer, 2012). In this situation, the citizenship courses were considered an imposed structure, out of step with some 
participants' concerns and study goals. The programme changes impacted on their choices and would affect the time it would take them to complete their project - their undergraduate degree. The change brought with it other financial and social implications. The changes were also believed to affect the quality of their degree. These students' attitudes of acceptance, resistance and rejection did not necessarily indicate a lack of agency; as in keeping with Domecka's (2017) finding, resistance, action, adaptation and 'reception' are responsive choices. The academic structures in place within the university had assumed people were suitably independent and unaffected by contexts outside of study (Baker, 2019), and that the changes to study programmes would be the same for everyone. The participants' reactions indicate that they were not and their choices were affected, but they were still able to respond to the situation.

Contrary to Archer's beliefs that people movements and national boundary changes in a postmodern world would be negative and disrupt traditional society (Archer, 2003), this study shows that contextual discontinuity between cultures can be normal and non-threatening, although it can be uncomfortable. Archer recognised change had differing impacts on individuals depending on how equipped they were to deal with changes. Hence, those with communicative reflexivity $(C R)$ traits evade change and autonomous reflexive $(A R)$ develop strategic approaches towards change, while those with meta-reflexivity (MR) traits subvert the structural enablements and constraints of change (Archer, 2007).

In Aotearoa/New Zealand, citizens encounter a range of other peoples, cultures and beliefs in daily life, even if they are not aware of it. Aotearoa/New Zealand is a young country, with a bicultural commitment and multi-cultural population. Many people identify with belonging to multiple ethnic identities, though their mixed ethnicity may not be evident to others in normal social interaction (University of Auckland, 2017). Aotearoa/New Zealand history has been influenced by on-going globalisation, successive waves of immigration and trade reliance on overseas markets since the first European occupation in the 1800s (Belich, 2007). The country does not have a monoculture with a single tradition or social class system on which Archer bases her theory of change; rather, Aotearoa/New Zealand society reflects the confluences and influences of encounters between Māori and non-Māori immigrants (K. Sullivan, 1994). The common-ness of cross-cultural encounters in daily life are evident in my participants' stories (Chapter 5.1). Shazz (MR-CR) had strong commitments to her extended families from European and Pacific Peoples heritage and a commitment to building a multi-cultural community. Elsie's (AR) lifestyle community drew in people from around the 
world, Karen (MR) had enrolled in a te reo Māori (language) course. Meanwhile, Jem (AR) was strategic in her approach when challenged as racist.

The evidence supporting Archer's reflexivity theory is based on UK studies where individual concerns of upward social mobility drove the reflexive projects (Archer, 2003). The traditional culture, class-system applications of reflexivity theory do not sit well within the Aotearoa/New Zealand context; the focus is not on moving between social classes. Reflecting on the nation's bicultural foundation, Nan, who firmly felt the country to be bicultural first, described the population as multicultural and able to continue to be open to refugees as there is "room here for more with the same values and care for the land" (Nan300). Her experiences with course group work had brought her into contact with new immigrants and new thinking, and she recognised the presence of common values. The aspirations of this group of Aotearoa/-New Zealand university students appeared not to be focused on social class mobility but on societal improvement through collective good, a finding similar to Bovill's (2012) study on work-based students studying in UK higher education.

In Aotearoa/New Zealand it is also common for people to move between cities and towns or other countries for work, leisure or study (Wilson, 2005). This transitory disposition means while national citizenship may be attached to identity, there are wider global and social experiences that influence an individual's consideration of structures and their effects on agency. However, strong ties in belonging to the land remain for both tangata whenua/ Māori indigenous to the land and tangata tauiwi/ non-indigenous people with strong environmental principles of caring for the land. Half of the original 31 participants identifying as Aotearoa/New Zealand citizens had lived overseas, were living overseas or had moved overseas during this study (see Chapter 4.4.2). A further three people identified as recent immigrants. Six participants discussed moving cities during the period of study for work or personal reasons. Thus, locality is not necessarily a strong indicator of connecting or belonging to natal contexts for this population. The passage of time and changes in place had allowed these individuals to step away from their familial backgrounds, choose those parts of their lives they wished to identify with and establish their own ways of living. As Deedee described in her written reflection, "with my role no longer being that of a powerless child, my sense of self is no longer controlled by that particular experience of home" (DeedeeWW100). The concerns and projects of the people in my study tended to be wide 
ranging, similar to Bovill's participants (2012), in looking at how they might contribute back into their increasingly diverse communities.

\subsubsection{On-going contextual discontinuity and agency}

Archer (2003) speculated that changes in circumstances "would precipitate a transformation in the agent's type of internal conversation" (p. 319). She argued "various combinations of contexts and concerns" (Archer, 2007, p. 315) produce different types of internal conversation, but her expectations were that a dominant reflexivity was normal. Jem (AR), Sage (MR) and Nan (AR) demonstrated this to be true in having a dominant reflexive mode. However, the dominant modes were temporary and changed between interview periods for others; for example, Nell (FR-FR-CR) and Deedee (FR+MR-FR-AR). Despite a broken natal background, prior to her study, Deedee had a strong identity based on her nationality, environmental concerns and her work in tourism. Her change in context and culture, with its contextual discontinuity, had left her adrift. The content of her reflexive deliberations was on her loss of identity and independence, how to connect with her new family and local community, and how to uphold her environmental concerns while economically constrained (see Chapter 5.1). In Deedee's case the structural influences were also political, physical and psychological. Deedee's experience was similar to that of international students in UK higher education, where the absence of trusted relationships pushed individuals towards autonomous reflexivity due to a lack of opportunity to discuss their concerns with others (Matthews, 2017). Unlike Archer's fractured reflexive participants (FR), who appeared to be passive agents (Archer, 2007, 2012), Deedee used the limited resources available to her but moved quickly from showing passive fractured reflexivity attributes to active autonomous reflexivity features. Goal-focused in planning her next steps, she had the self-determination to handle complex situations and with time, small manageable projects were considered, set up and achieved.

Contextual discontinuity from an individual's natal environment can occur, but it appears possible to build a secure replacement as a person moves along their life course. Nell's experiences seem to suggest that she gained agential capacity (Biesta \& Tedder, 2007) in constructing a new life with her partner. Disruption occurred in Nell's teenage years when her parents' marriage split, they took different paths and Nell felt left adrift. Nell considered her mental health conditions as a permanent part of her life leaving her unsettled. Nell's feelings of powerless to make decisions or act for herself is typical of some individuals with an 'impeded' fractured reflexive state (Archer, 2007). Nell saw herself as a passive agent. 
She initially lacked conversational partners and personal emergent powers. The fractured reflexivity traits (demonstrated in the first two interviews) meant she was unable or unprepared to engage in internal conversations about herself and her circumstances (Archer, 2003), and therefore unable to act for herself in looking for a place to belong. Besides her studies, Nell had no other projects and was not prepared to consider possibilities. Over time, having entered a stable relationship, Nell appeared to reflect more on herself and her interests (Archer, 2003), rather than just seeing herself as unwell. She became active in planning her future.

As Nell and Deedee's reflexivity changed over time in response to changes in personal and social circumstances (see Chapter 5.1), it seems likely it will change again should circumstances change. Thus, the presence of a dominant reflexive role may be temporary and located within a specific context and operational modus vivendi. The recognition of alignment between communal possibilities for action and personal values appeared to be an important trigger in Nell and Deedee moving to a new mode of reflexivity.

\subsubsection{Changes in circumstances and modus vivendi}

On-going changes in immediate circumstances, as well as wider societal change, can alter an individual's sense of self and their reflexive commitments. Life is lived out in the natural, practical and social orders where personal identity is formed in the social order and social identity in the practical order (Archer 2003). Even to be able to think about one's identity is a reflexive activity (Rossiter, 2007). Feelings of powerlessness in dealing with family issues may be the result of reflexive thinking about a situation and from recognising a shift in the nature of one aspect of a person's current identity. As a parent of a young-adult family member in a difficult situation, Karen realised her power to intervene on her child's behalf had changed. Her modus vivendi was undergoing change. Her relational or social self (Oyserman et al., 2012) as a mother formed a strong part of her identity. The stability of knowing this brought meaning to Karen's activities. Institutional separation from this role had temporarily disrupted Karen's sense of identity and agency, and her reflexive focus. The change in social conditions impinged on her agency. With time she had accepted the situation and re-envisaged her thinking on her roles, recreated a new image of herself and her parental role, and where that fitted with her view of her future self. The structural contextual cues of her relationship had altered (Rossiter, 2007), but her agentic view of her wider relationships was enhanced. 
Archer sees social identity as a subset of personal identity and agency, where personal identity is developed internally and social identity developed as the person must deal with the social and cultural structures they encounter (Archer, 2007). As a primary agent, Karen had new awareness of the social limitations acting on her choices as a parent. She still had a strong parental role, but her active role had changed. Overall, her view of her identity had adjusted to incorporate understanding of structural aspects of the wider world. As Karen stated at the end of the courses,

I think I'm still fairly secure in terms of identity - mother wife, professional person with a degree hopefully... I would expand my identity to include a global citizen, a person who lives in this world shared with millions and millions of others all equally living in this world. We're all entitled to be here and I still ... believe strongly in the issues of fairness, but perhaps being a lot more aware of it than I was before, and more aware of where my place is in it. That I am one member, and that we are in this huge community of people around the world all living our lives. But mostly believing that we are individuals when really, we are connected individuals. We just might not know where and how that connection is yet (Karen300).

In her consideration of the structural and personal values of citizenship, Karen found the freedom to act and shape her immediate situation and to think about her contribution to wider social change (Giddens \& Cassell, 1993). Karen's social resources remained the same, but she was able to connect her current actions to long-term social good. While continuing to grow her family, Karen could envisage herself acting in different areas. The layering of her reflexive thinking had provided an awareness of different opportunities to act on the values which formed her identity.

\subsection{Internal conversation and social interactions}

All participants associated their internal deliberations to some extent with Archer's definition of internal conversation as self-dialogue and as an "emergent personal power" (2007, p. 3). However, my study suggests the input of other ideas is an important trigger to deepening reflexivity and identifying ways forward. All respondents indicated they compared or contrasted the citizenship course ideas presented with their own concerns and the views from their social and study networks in their internal conversations, but at various levels of 
intensity. Thus, it appears that their agency is interdependent and influenced by others, in keeping with Burkitt's (2016) argument that agents do not act alone in their reflexive thinking. While they would mull-over or examine a presented idea or reading, the participants would then seek out others' understandings, so they could critique the information from multiple perspectives. Archer acknowledged (in countering King's (2010) description of her agent) that people are not solitary beings, but she continued to diminish the influence of social conversation on agency while elevating the influence of structures (Archer, 2012). What was important to the participants in my study was that the process of mulling-over was an acknowledgment of their social connectedness and that others held different perspectives from their own. What differed was whether internal conversation was a continuous spontaneous feature, used irregularly but purposefully, or episodic and even carefully controlled to reduce the emotional effects of reflexive thoughts (Holmes, 2015).

\subsubsection{Individual or communal needs and concerns.}

On-going internal conversation was a strong and routine reflexive process for managing life priorities, organising one's thinking, gathering ideas and debating possibilities. Five case study participants, similar to those in Archer's Coventry interviews, assumed internal conversation was universal and everyone's experience of it was the same (Archer, 2003, p. 161). However, in my study I found there were two sub-groups based on whether the focus of their internal conversation was their own views and needs (individual) or other people's views and needs (communal). This is something only alluded to by Archer, in those with communicative reflexivity traits checking and ensuring their concerns fit with their natal group as they work to maintain their self-worth (Archer, 2003). Self-focus is the defining characteristic of autonomous reflexive thinking and behaviours.

Meta-reflexives uphold their ideals on social improvement; thus, their focus is on improving the lives of others. Three female participants with complex work-life-study situations (Karen, Shazz and Sage) focused on others. They had many identity roles, including parental and/or family relationship roles, work, volunteer and/or community roles, being a student and a citizen. Biographical studies (Caetano, 2017a; Mrozowicki, 2011) also identified this communal-individual dualism as a distinguishing feature in their models of reflexivity processes. "Communitarian" covers an individual's place within their extended family, family ethos, roles and use of resources, but was typical of those living within a small geographic area (Mrozowicki, 2011). In my investigation the communal focus of reflexivity covered extended family but moved beyond the immediate to incorporate specific groups 
(beneficiaries, senior citizens, refugees) and the social groups participants identified with (tangata whenua, Pākehā, students, prospective employees).

Roles within social groups provide social identification in which actors monitor their key values, aspirations and concerns in their self-dialogue in the natural, practical and social worlds (Archer, 2007). These roles are reinforced in an individual's interactions with their social environment, in the language they used to describe themselves, and provide a continuous sense of self and personal identity based on their activities (Archer, 2007; Berger \& Luckmann, 1966; Luckett \& Luckett, 2009). Two participants in my study with autonomous reflexive qualities (Deedee and Jem), who focused on their own lives, identified with a smaller number of roles. They presented themselves in interviews, on discussion forums and in other writings as students or by their past employment or civic activism. For example, Jem's described herself as a BA student, single, intelligent high achiever with strong opinions based on life experience. Uncharacteristically of Archer's autonomous reflexivity mode, those with autonomous reflexive modes in my research project backed their own judgment and worked on accommodating and respecting the expectations of others. This was conditional on others' concerns fitting with their concerns; they did not prioritise others' concerns over their own (Archer, 2003). They were skilled at balancing these two spheres in their inner dialogue. However, where family might have provided an emotional barrier between her self-focus (Mrozowicki, 2011) and other reference points for interactions with the world, Jem's contextual discontinuity and contextual incongruity meant her interactions with others were raw and her concerns absolute.

\subsubsection{Prioritising others' needs first}

Despite claims of increased individualism and those with meta-reflexivity tendencies being Ioners (Archer, 2007, 2012), understanding others' concerns and needs was a priority for Karen, Nan and Sage. If a situation was unclear or not understood, then the various angles and arguments were examined through holding discussions with others until they clarified the issue at hand. They might then listen to the voice of those others in a semi-autonomous, but conscious manner in their minds, their dialogical unconscious (Burkitt, 2010). Only then could these individuals make their decision on how to act. They sought the opinions of those in their near social networks - family, friends and regular groups. Where immediate exchanges in the local community were limited for Deedee, she extended her consultation to contacts on social media platforms. There was a strong sense of optimism and solution seeking in these external conversations; participants appeared to be strategic and focused 
thinkers with a modus vivendi open to reconsideration (Dismore, 2016). Possibilities for enacting or applying values were considered and the benefits and constraints examined in conscious thought.

Similar to attributes shown in Caetano's (2017a) pragmatic reflexivity, practical aspects of life were the focus of Karen's, Nan's and Sage's reflexivity. Thoughts and discussions about themselves and their circumstances were orientated around family and work priorities, schedules and requirements. Those who prioritised others first could identify the resources needed and manage present resources towards the things they held as important in their lives. Budgeting of resources - an important part of internal conversation (Archer, 2007) was also important for those with strong family commitments. Financial resources were a primary concern. The availability of funds entered into thinking primarily as a constraint on what could be achieved or could only be achieved in the future if circumstances were to change. Despite having autonomous reflexive characteristics, at a practical level Nell demonstrated that the possibilities for her actions could be considered collectively in external conversation and spread through social media. Social media provided a window to another world of likeminded people for Nell to share her views. In this case, technological advancements offered a stronger possibility for connectedness and social change than Archer and her contemporaries had considered.

\subsubsection{Dove-tailing identity and citizenship}

Initially, most participants' perceptions of themselves as citizens were associated with place and identity. Place was considered in terms of the 'near' as an individual or member of a community or family, and 'far' membership in an ethnic or national group, or as a global "citizen of the world" (Elsie100). Consequently, their values were associated with the concerns of those groups.

As they moved through their studies, internal conversation of identity and identity roles changed from 'who I am' to run across both personal and social roles of 'where I fit'. From an identity-based motivation perspective, personal identity roles characterise images individuals have of their future self while social roles affix a place (Oyserman, 2004; Oyserman et al., 2012). Sage discussed her personal role as a mother studying to bring up "conscious children" (Sage100), attributing this to the actions of a responsible citizen - a social role. This is also an example of meta-reflexive characteristics working towards establishing a modus vivendi where an individual, or actor, prioritises their ideals, aware that 
others do not hold the same values (Archer, 2007). Shazz aligned how her natal immigrant background had influenced her thinking on how she could support new refugees, and how it was helping her to contribute to her local multi-cultural community. Her social and personal roles dovetailed. Karen's social and personal roles also melded in the mixed age groups she volunteered and worked with. Her goal was to create a better environment for all the age groups she interacts with - characteristic of her meta-reflexivity. Although struggling with her identity in her current circumstances, Deedee also expressed social responsibility in her "continuous consciousness" (Deedee300) of her contribution to and responsibility for the global environment. All four participants wanted to live up to their values and pass them onto their families or others in the community. In identifying where they fitted and their future selves, these participants were more reflexively focused on the opportunities for active citizenship projects that were consistent with their internal conversations.

These people were firmly connected, despite predictions that the loss of social structures and socialisation processes with distance from traditional ties of family and locality would leave them adrift (Archer, 2003; Beck et al., 1994; Giddens, 1991). Rather than the "dynamics of the transformation of society" (Caetano, 2017a, p. 1) overwhelming social structures and requiring Deedee and Karen to be more reflexive, they are both reflexive and connected to familiars and community even if the longevity of time is missing. Karen volunteered in schools and church, participated in jury service and looked for opportunities where she could contribute after relocating to a provincial town. While focused on the immediate context, she saw herself embedded more widely - "we need to be aware of what's happening in our community, our world and our society and close by, as it all impacts on the whole" (Karen300). Shazz and Sage were locally well connected to their family and communities, and aware of opportunities for further involvement. There did not appear to be any social transformation that required them to be more reflexive as Archer (2003) proposed; rather, their heightened reflexive awareness seems to be a personal characteristic.

Identity formation is a reflexive process that continues over time as personal circumstances and concerns and social networks change. Identity theorists Stryker and Burke (2000) posit that social structures are necessary in identity formation and on-going self-awareness. The connection between structure and agency is a part of identity production and verification. Over time, encounters between structures and agents change. Consequently, the interaction changes. However, Archer believes social identity to be a substructure of personal identity, where the primary agent compares "me" with the social "you". If there is a change in position 
in society then there is a change in identity and instability in reflexivity occurs (Archer, 2000). Deedee's early disrupted natal upbringing and contextual incongruity meant she had to develop new identity reference points. Her experience appears to fit the positions of both Archer (2000) and Stryker and Burke (2000). However, cultural differences in identity and residency between Aotearoa/New Zealand and Deedee's adopted country, and her pregnancy have had a much bigger impact - hence at the second interview her loss of identity and features of fractured reflexivity were evident. As Archer (2000) explained, social roles must align with personal commitments and concerns to produce a modus vivendi but these are not always controllable. However, Deedee was demonstrating agency through consciously taking small steps in sharing her values, building environmental awareness and caring for her new community and settling into her new identity role as a mother.

My small-scale study found strong variability in the nature of each individual's internal conversation, how it was used and how useful it was in defining possibilities for action. It was dependent on social networks, and internal deliberations were impacted by external circumstances. It is possible that the reflexivity profiles of this group are unusual and might not be found in a wider cohort. Future research on the nature of individuals' internal conversation with a wider cohort would verify if this was so.

\subsubsection{Confluences in personal reflexivity and citizenship}

In analysing the contribution of external conversation and writing to internal deliberation, I decided to compare Caetano's personal reflexivity types (Caetano, 2017a) with my study group. I wanted to find another lens, which included the analysis of writing and other conversations (Caetano, 2017b), to consider the reflexive characteristics that were prominent in my study.

The context for reflexivity in my study was citizenship and its values rather than the decisions made over variable lengths of life-course, which was Caetano's focus. Whereas, Caetano was interested in the variability of family backgrounds and social class on life projections, social class was not a consideration in my study although socioeconomic circumstances do feature. Caetano's group ( 21 to 80 years of age), varied in educational levels, occupations and levels of privilege (Caetano, 2017b). My participants (ranging in age from 20s through to 50s) were all undergraduate higher education students in a country with relative stability rather than Caetano's context of a society that had undergone dramatic political and social change. Many of Caetano's subjects had lived within or close to their childhood home for 
most of their lives or for generations (Caetano, 2017b). In contrast, the participants in my study were more mobile, travellers, or open to moving to follow their career paths. They were less likely to have links to a long history of local and familial traditions.

Although individuals in my study fit some characteristics of Caetano's personal reflexivity (2017a), there was inconsistency across all the types. For example, six of my participants had high qualifications and strong reflexivity features in different contexts yet their match on the remaining characteristics within Caetano's typology was variable. Jem showed the strongest match with Caetano's self-referential type matching all of the seven characteristics defined. Jem was focused on the present (maintaining her high grades), her accomplishments and long-term goals, constantly questioning ideas and activities around her, identifying and rationalising the choices she had made so far, and what she could and could not control in her life path (Caetano, 2017a). However, where Caetano's selfreferential group had on-going social contacts that reinforced their views, Jem did not appear to have a social sphere which was reinforcing her views. This difference makes her more consistent with Archer's autonomous reflexivity mode, as she was highly independent and believed she had the power to accomplish what she sets out to do (Archer, 2007).

Caetano uses the length of a project to differentiate between reflexivity characteristics (2017a). However, the goal of getting a degree was a long-term project all my participants had committed to and were pursuing, despite interruptions or being uncertain about how they would use the qualification. Participants also had short term or intermediate projects many which were being acted on immediately. Using project length to distinguish between Caetano's reflexive groups was not possible.

Continuous reflexive thinking on the values of citizenship in internal conversation, however, was reflected in participants' external discussions and writing, similar to Caetano's findings (2017b). Like the participants in Caetano's (2015a) study, in my study external conversation in specific contexts provided opportunities for mediating concerns and priorities. In these conversations, expectations, goals and projects could be negotiated with others (Chalari, 2007). Similar to Luckett and Luckett's (2009) findings with first-year South African higher education students, the participants in my study also externalised ideas to test their views and to hear the views of others; in doing so, they built their confidence. As corporate agents (Archer, 2007) in discussion with others, participants could critically reflect on their personal situations and strategic possibilities for engagement. My study demonstrates there was a 
strong interconnectedness between these diverse higher education students' reflexive ideas and social interactions in their discussions and writing, as there was in their internal conversations (Chapter 6.3).

\subsection{Dual or multiple reflexivity modes}

Enjoyment in learning in higher education can be pivotal in understanding how wider enablements and constraints are framed in reflexive thought and acted on (Dismore, 2016). From her various studies, Archer proposed adults used one dominant reflexivity mode at particular points in their lives following their social development and aspirations to move up the British social class system (Archer, 2007). This was based on the assumption social mobility was the driving force behind individual and social changes. In her study, Archer concluded that university students progressed from communicative reflexivity to show autonomous reflexivity, pinnacling as meta-reflexivity over the period of their undergraduate degree as a result of their increased education (Archer, 2012). This development was the result of moving from their stable social contexts to encounter a different range of values through intermingling with people from other backgrounds and social classes. Having developed meta-reflexive qualities, they were then considered to have broken away from their natal backgrounds and the associated values and concerns maintained there.

As I have shown in Chapter 5.4, Archer's single dominant mode of reflexivity and progression of reflexivity over time is not supported by my study. In my research project, age range as an indicator of participants' dominant reflexivity and life stage, does not concur with Archer's (2003) proposition. Life and roles within stages can vary in sequence and length, as demonstrated in Jem's increasing autonomous reflexivity over the study period as her family's reliance on her had shifted, and her life goals were elevated. These changes in wider social and personal circumstances were found to have a greater effect on reflexivity and reflexive mode than their educational level. Baker (2019) also questioned whether dual modes of reflexivity were related to age, experience or context.

Five participants demonstrated characteristics of using two reflexive modes at any time over the study. Kane showed the continuous use of two modes - meta-reflexivity and communicative reflexivity - across time. His meta-reflexive characteristic of an "exploratory outlook towards the social" (Archer, 2012, p. 201) was in upholding the values from his upbringing. Rather than separating him from his natal, communal-based values, Kane had 
embraced and committed to them, acting on them after considering ideas on global rights presented in the citizenship courses. His communal values are the common factor in his employment, his studies and plans for a career in enacting his global values. Whether Kane's upholding of these values was the result of social conditioning (Archer, 2003) or habitus (Bourdieu, 1984) could not be determined in this study. A full biographical study may have revealed more. Kane's meta-reflexive traits reflected his priority in bringing about meaningful action and social change. Combined with Archer's communicative reflexive (Archer, 2007, 2012), he consulted, checked and tested his thoughts against his siblings' common values. In contrast, Elsie displayed both autonomous reflexivity in her linear thinking of her commitment to her community and working towards her own concerns, and meta-reflexive traits in critical detachment from wider social concerns (Archer, 2012). However, it was not clear whether this reflexivity combination affected her agency.

Several studies have shown that higher education students used more than one reflexive mode (Baker, 2019; Bovill, 2012; Dyke et al., 2012). My study of diverse students (ranging in age from 20s to 50s) does not support Baker's findings of traditional higher education students (16-19 years) demonstrating multiple reflexive modes in decision-making over very short periods of time, dependent on the topic being discussed in their courses and who they were talking to (Baker, 2019). In the diverse students in my study, there does not appear to be a shift based on rapid decision-making. Rather, this study supports Dyke, et al's (2012) suggestion of the presence of dual-modes of reflexive attributes as a response to shifting circumstances. Changes in Deedee (FR/MR-FR-AR), Shazz (MR/CR-MR/CR-FR) and Karen's (MR/CR-MR- MR/CR) (see Table 5.5 in Chapter 5.4) lives and changes in their understandings of their situations implies the presence of a "dynamic relationship between individuals and changing circumstances and how individuals adopt different approaches to reflexivity to help them navigate their way through emotional and career pathways" (Dyke et al., 2012, p. 846). Troublesome events affected and constrained their agency, and this intensified their distress (Matthews, 2017); however, the presence of the fractured reflexivity mode was temporary. Dyke saw the temporary nature of reflexivity as consistent with Archer's view of emergent personal properties that can change over a life-course, and therefore the co-presence of reflexivity modes should be expected (Dyke et al., 2012). The limited participant numbers in my study do not provide a clear indication of this, it will be interesting to see if the remaining 22 participants who have yet to finish their 300-level course have single, dual or multiple reflexive modes. 
The participants in my study also "reflexively interpreted their circumstances and navigated courses through them" as their circumstances changed (Dyke et al., 2012, p. 847). However, rather than the change extending their reflexive modes as Dyke found, Karen and Deedee became more focused and reverted to the characteristics of a single and more dominant reflexive mode as disruption in their lives occurred. Once Karen's situation and routine settled between interviews, she returned to using both meta-reflexive and communicative reflexive traits again. During the period where she needed to focus on the issue at hand, her energy and her thinking on her studies focused only on what was necessary. Deedee, similarly, used autonomous reflexive attributes in single-mindedly looking for solutions to the structural implications of her citizenship residency status. In keeping with her autonomous reflexive characteristic she could experiment in her thinking and action without worrying others (Archer, 2007). This intense focus was also evidenced in Elsie's move to autonomous reflexive characteristics after her relationship broke down, and she realised that she would need a job to support her children. In all three cases, there appeared to be a possibility that parental roles and responsibilities moved these individuals to one particular reflexive direction in making plans to protect or support their children. The restraints of their situations were external and needed to be negotiated carefully if they were to have the agency to act in their children's best interests. This is an interesting development on shifts in reflexivity modes that requires further investigation.

Three participants did meet Archer's expectations of using traits of a dominant single reflexivity mode where "the dominant mode of reflexivity practiced by singular subjects did not appear to be psychologically determined" (Archer, 2012, p. 16), but stability in the structural and cultural characteristics of their background influenced reflexive processes. Living and studying situations and goals remained constant for Nan and Sage (both demonstrating meta-reflexivity characteristics) and Jem (autonomous reflexivity traits) as they completed their degrees. Sage's meta-reflexivity mode features appeared to weaken slightly, as she recognised her current social and financial constraints would remain upon completion of her degree. To widen employment opportunities, she would have to leave the only stable accommodation she had had in twenty years. This potential change had her troubled as it compromised her future citizenship in making a difference in the lives of the social welfare beneficiaries she interacts with in her local community. Meanwhile, Nan's meta-reflexive characteristics remained strong despite being in circumstances similar to Sage. Nan was open to relocating to find employment. Nan and Sage held societal 
improvement ideals and were working to uphold and act on these. Their depth of life experience and resources established with time (although limited) seemed to help in forming the manifestations of a single reflexivity mode (Baker, 2019). This finding agrees with Dyke's proposal that people should not be seen as "harbouring one type of reflexivity", but reflexivity should be "seen as approaches that are context specific, where individuals are capable of multiple approaches" (Dyke et al., 2012, p. 846)

In their biographical studies of life choices over time, Caetano (2017a) and Mrozowicki (2011) suggest that marginalised people's social circumstances and political influences play a wider role in their reflexive thinking and responses than Archer's methodology incorporates. Structural traditions and societal views of people who receive social support is often that beneficiaries are passive agents. This societal view may be integrated into one's consciousness and may be a strong psychological constraint on agency. Another consideration in Aotearoa/New Zealand education is the discourse of Māori and Pacific Peoples underachievement (Ministry of Education, 2014; Ministry of Social Development, 2016). In this, a deficit view positions Māori and Pacific Peoples students as passive agents requiring additional support (K. Sullivan, 1994). However, Nan's actions in continuously pursuing possibilities for action and acting on projects suggests she was Māori and an active agent, as was Deedee, a former social welfare child. Further investigation of the reflexivity and action in the remaining participant group who receive social support or identify as Māori, Pacific Peoples or refugees may provide further understanding on this.

\subsubsection{Cross-checking reflexivity modes}

My study's findings of the presence of dominant modes, dual modes and shifting reflexive modes over time in the nine respondents requires further exploration. I hope this may be possible in the near future as the data from the remaining original participant group is analysed. Four aspects are worth exploring further- what are the situations of those with stable, dominant modes? Does parental role result in fluctuations in modes for all parents? Are particular shifts in traits between modes associated with similar changes in circumstances? Or, do the reflexive modes demonstrated by Māori and Pacific Peoples students fit or belie the deficit 'passive agents' assumption? 


\subsection{Controlled reflexivity}

Three people in quite different circumstances demonstrated the use of a form of controlled reflexivity for specific purposes. They exhibit some of Caetano's (2017a) resistance reflexivity characteristics in developing and using their reflexive competencies just as needed at the internal, external and written conversation levels. External conversation where the self is presented may be strong or weak. Elsie, revealing her autonomous reflexive traits, had a strong personal ethic and values (Archer, 2007, 2012), and selectively shared her views. However, her internal conversation was limited, managed and centred on her own priorities and experience. Her use of the embodied conversation practices followed in her community was a higher priority than focusing on herself. As a result, writing was her main reflexive process of citizenship concepts, used to focus, order and deliberate ideas within a formal structure.

Jem also liked to be in control (Archer, 2007, 2012). Therefore, writing was a highly functional space to present her ideas and to meet her priority of maintaining her high achiever status. Her own perspective was central to all internal and external conversations. These findings support arguments (Caetano, 2017b; Chalari, 2007) for the further examination of the role of external conversations and writings in considering reflexive deliberations. In higher education, the main purpose of written assessments is to gather specific information on each individual's knowledge for grading; this matches the focus of self-determined autonomous reflexives. Rather than using the critical reflections to make real-life connections and applications of their knowledge with their personal values and goals to make a difference in society, as those with meta-reflexive tendencies do, autonomous reflexives can distance themselves and their emotions. In their study of online post-graduate students' reflexivity and engagement in their studies, Kahn et al. (2017) concluded those who actively engaged reflexively 'flourished' compared with those with restricted reflexivity. However, controlling reflexivity and the emotions associated with reflexivity (Holmes, 2010, 2015) could also be seen as an agentic means of coping.

Prior biographical events do appear to have an impact on reflexive commitments and the values individuals are prepared to commit themselves to (Archer, 2003). Nell's internal conversation and external dialogue were both constrained and managed to maintain her mental health and lessen her anxiety which is her primary concern. Initially, she appeared resistant to developing reflexive thinking as she had not been able to control difficulties in 
her past life, and the impact they have had on her life (Caetano, 2017a). Once her personal circumstances changed, she engaged in more internal pondering and presentation of her ideas on social media. The orientation of her reflexive thoughts was now on her present and future self rather than past experiences; the constraints of guarding her emotions were loosened. Nell was able to acknowledge how writing discussion forum posts and stepping outside for a cigarette had become external spaces for internal reflexive thinking and she was now clearly showing the attributes of Archer's communicative reflexivity mode (Archer, 2007, 2012).

The use of controlled reflexivity ensures the actor balances their concerns against their projects and goals. They manage and contain both their internal and external deliberations. The social interaction itself is not important to Elsie and Jem as they preferred to consult with those with similar viewpoints and authoritative positions. In turn, they shared their thoughts in select environments where their views count. However, sociability in the interaction had become an important part of Nell's reflexivity. The findings on controlled reflexivity brings new light on the use of reflexivity to achieve personal goals and emotional stability. Controlled reflexivity reflects different levels of agency. The exploration of the effects of the wider social processes and experiences of this diverse group of higher students, contrasts with the findings of Archer's (2012) study on a homogeneous group, and offers possibilities for a broader understanding of how reflexivity affects agency.

\subsection{Writing extends the internal conversation}

Internal reflexivity is not the only means of mediating concerns and priorities (Caetano, 2017b). In the citizenship courses, writing creatively was used to generate reflective schemas to examine thinking. It assisted Deedee, who was socially isolated, in processing and laying out her unspoken current concerns and priorities regarding her own citizenship status. In her coursework, the feedback from her tutor provided reassurance that (as a new student) she was meeting the university expectations and her reflections were valid. The critical reflections also encouraged agentic practices in participants demonstrating the connections between their own ideas and life experience (Kahu \& Gerrard, 2018). This challenge was also taken up by Karen, Sage and Shazz in demonstrating clear associations between the course ideas and their own circumstances. However, by the end of their degrees, Sage and Jem focused on the functional purposes of assessment writing to achieve grades disregarding any connections to personal reflections. Thus, the use of 
writing as an extension of internal reflexivity's debates in citizenship understandings produced variable results.

The use of self-reflective commentaries is not a reliable indicator of reflexive development, nor of the change that academics might be seeking in their students as a consequence of their studies. Frequently self-reflection is used as an experiential learning process to engage the learner in connecting knowledge and its application, focused on feelings and responses to issues (like racism or equity) or as an evaluation of learning growth, including what has or has not changed (Macfarlane, 2017). This "emotional performativity" requires students to publicly demonstrate the way in which learning has taken place (Macfarlane, 2017). It is reliant on the student's written academic ability and willingness to talk about their values or feelings. As this personal and often private information is to be judged by another, what is revealed may be monitored by the person. Like Jem and Sage, learners may take a functional approach to assessment or respond based on their prediction of what the tutor requires in an answer. Consequently, self-reflective written assessments and discussion forum posts such as those used in this study need to be used carefully. Without some form of triangulation (Bazeley, 2013; Ritchie et al., 2014) their value in determining modes of reflexivity is limited.

\subsection{Higher education students' reflexivity}

The participants in my study supported the notion that reflexive consideration of themselves, circumstances and possibilities to act took place within their inner conversation. In addition, I found this contemplation was informed by their external conversations and their values, and consideration of their personal circumstances. My research was situated in Aotearoa/New Zealand a bicultural country with an increasingly multi-cultural population. The Aotearoa/New Zealand context is more representative of an integrated society that reflects the effects of globalisation (Belich, 2007) than those envisaged by Archer and her contemporaries of cultural invasion on traditional societies. The question this raises is how citizens consider and settle differences in cultural and social values as they encounter them in society. Archer's critical realist theory of morphogenesis, reflexivity and internal conversation (Archer, 2003, 2007) provided a useful tool for understanding the structureagency relationships experienced by individuals. However, as discussed in Chapter 2, I believed Archer's approach lacked sufficient consideration of the wider social elements and interactions which inform people's decision-making in reflexive processing. This research 
sought to address this gap by looking at how the social affected higher education students' reflexive processes as they considered their values and possibilities for acting within the complex realities of their lives.

In Aotearoa/New Zealand post-compulsory education, the development of personal citizenship characteristics and skills to promote social cohesion, equity and democratic values is seen as a necessity for twenty-first century life and employment (Ministry of Education, 2014). In this section, I present my tentative thoughts on higher education students' considerations of their citizenship values and concerns. These self-selected participants were members of a wider cohort of the diverse new majority of higher education students studying at university. My framework offers my research thoughts on the complexities of the internal conversation revelations of the structure-agency relationship of this group. I propose that reflexivity is a more complex relationship between the social interactions, personal contexts and opportunities for agency than that presented in Archer's typified modes of reflexivity within traditional societies. As Caetano argues, a person can be highly reflexive in certain social contexts "but strongly guided by structural constraints in others", and "activation of reflexivity cannot be thought of as a uniform and transversal process" (2017a, p. 68).

\subsubsection{Structure-agency in individual reality}

Understandings of individual reality and the interplay of structure-agency relationships inform higher education students' reality. Archer's methodological dualism provides a means of recognising the influence of structures and agency as separate but related parts of individuals' realities in the natural, practical and social orders (Archer, 2003). On the other hand, the recognition from social constructionists of the social tensions and differences of people's view of reality (Berger \& Luckmann, 1966) balances Archer's (2000) reliance on structures in social conditioning in rejecting Bourdieu's habitus. Society's structures have properties or features which influence an individual's freedom to act within and on society. However, as argued by Archer and demonstrated by the participants in my study, this influence is neither homogeneous nor static. This variability in context and the nature, use and characteristics of reflexive processing means any presentation of 'typified' modes reflexivity is inherently unstable.

This variability is reflected in the different structural influences on participants. Economic structures and resources are mirrored in discussions on social position, opportunity for 
employment or acting on beliefs and family commitments. Economic structures formed a barrier for Sage, who couldn't afford to relinquish the stability of her state-funded home to pursue employment in other regions. This illustrates the decisions individuals need to make in weighing up their choices and priorities. This contextual incongruity where as a metareflexive she couldn't realise her dreams (Archer, 2007), was a site of immense frustration for Sage as she felt she was letting her society down. In contrast, Kane's financial position permitted him to develop and implement a project away from home to pursue his personal commitment to his values. Family and cultural structures were reflected in conversations on maternal and paternal roles and individual responsibilities. For most participants family structures were both enabling and constraining. Parental roles offered possibilities for Deedee and Shazz to engage with their local communities but also restricted their ability to act in their wider cultural structures. However, both were aware that this disjuncture should change over time (Cieslik, 2006). Nan's role as tangata whenua, with cultural responsibility for the land, and her concerns for wider whanau/family drove her opportunist mindset to look for means by which she could act on her values. Variability in encounters with political structures - in petitioning local and national governing bodies - left some (for example, Karen and Nell) uncertain, while others (for example, Nan and Jem) flourished. These structures are referenced in both their internal conversation and external discussions as individuals try to 'make sense', to identify or position themselves within society, and what is within and outside their capabilities and resources.

One way of looking beyond the constraints of structures is in dreaming or imagining what could be achieved. Thinking about the possible or future self (Rossiter, 2007), in what can be emotionally positive projections (Burkitt, 2012), has the potential to bring about actions that quickly move beyond individual lives. The reflexive imagining of the self beyond the structures of higher education and employment can provide a mechanism for seeking social solutions to the complications that have arisen from rapid globalisation. The development of students' agency through higher education can be a partnership in learning between students and staff (Felten et al., 2019). In balancing social agendas against economic agendas, the entwined nature of structure and agency remains, but it should provide a context which offers diverse people greater opportunities to act on their values, and to flourish (Kahn, 2017). They may not have the freedom to act at will but can act in small and bigger ways on their values within the resources and opportunities that exist. 


\subsubsection{The importance of context}

Life contexts influence the opportunities for social interaction and are therefore more important for reflexivity than identification of dialogic partners. The context in which reflexive thinking takes place influences the possibilities for action. People as agents have varied contexts within which they operate. The stimulation for reflexive thought differs according to where the person 'fits' within the social world and in the 'intra' and 'inter' variations (Caetano, 2019) in the different aspects of their social world (for example, in roles as workers, financial providers, friends or as a student). The exercise of reflexivity through external conversations indicates people do not see themselves as isolated from society but operating in multiple social networks where they must consider their actions (Caetano, 2019).

\section{Personal context}

Within their varied settings, individuals' dream of possibilities, plan for eventualities and decide on their approach to the future. In doing so, agents have the potential to endorse, replicate and modify existing social structures in their daily interactions. Three participants Nan (Māori), Elsie and Kane (Pākehā) saw the structures and actions of living in community as a norm. Nan was brought up by her parents to acknowledge her culture where Mātauranga Māori knowledge and culture connects all Māori people through their ancestry to Ranginui sky father and Papatūānuku earth mother. Nan upheld the communal values of her parents; she identified with both the land and people in her interactions at both local and national community level. Nan's wider vision meant she did not see structural features as personal constraints. Rather, she constantly thought about the possibilities for using various governance structures to bring about change to improve her community. Kane shared a similar position to Nan but with a strong commitment to global communal values and priorities. Their contexts reinforced their values and wider concerns, similar to those enacted in Elsie's chosen lifestyle.

Internalised understandings of social structures are open to challenge and reconstruction when the individual is not fully committed to them (Berger \& Luckmann, 1966). In their reflexive deliberations, people exercise their personal agency to decide what they think about external reality (Archer, 2003) and how they will respond. To understand others' viewpoints in a rapidly changing world requires the socialiser to be a "strong evaluator", reflexively selective about their concerns and activities and those of others in their relationships and interactions (Archer, 2012, p. 124). Karen was fully committed within her 
social reality - a relational world of family, friends, work, community and faith contexts - but she was also a strong evaluator and open to others' views. The richness of her external communications helped her to recognise how structures and agency worked in multiple social contexts. It is through these sorts of interactions that participants in my study defined, negotiated and reworked their goals and projects (Caetano, 2017b). While her various social contexts have intersecting and disparate values and priorities, it was Karen's personal commitments and values that tied these layers together.

The richness of past and present contextual layers was different for each participant. This influenced their perspectives of the past, current engagement and agency, and orientations to the future (Biesta \& Tedder, 2007). However, it was also dependent on their goals, relationships and emotional connectedness to their current environment. Thus, it was these intersecting and disparate contexts and the academic environment which provided the reference points of their reflexive thinking.

\section{Higher education context}

Higher education, the context in which the stimulus for participants' reflexivity took place, reflects particular structural traditions and expectations of students. The western university system encourages the individualistic characteristics of the autonomous reflexive mode (self-motivation, self-regulation and self-promotion). Self-development is the reflexive project (Biesta \& Tedder, 2007) and social replication of existing social structures is the goal (Ashwin, 2008). An extended period of university study can also transform the individual, in such a way that they can develop holistically - in critically examining ideologies, contesting hegemony, power, alienation - and contribute back to society in upholding democratic principles (Cranton, 2006). However, rather than preparing students for a dynamic and social global environment, the current focus of western higher education appears to be following monetarist goals, with a focus on improving wider economic and political agendas (Macfarlane, 2017).

Despite these wider national goals, the expressed focus of all nine participants' reflexivity in my study was on societal improvement rather than employability or economic values. While the online learning environment may have reduced the opportunity for students to interact directly with academics and the university environment, it helped my participants retain their connections with their community and families. As Caetano (2017) elaborates, individuals may have "different levels of reflexivity in different social contexts because some settings 
can do more than others to stimulate the development and activation of reflexive competences" (Caetano, 2017b, p. 4). If they were 'traditional' on campus students, it could well be their reflexive commitments would reflect those of other university students as found in studies by Archer (2012), Luckett and Luckett (2009) and Cieslik (2006), or individuals moving through life transitions (Barratt et al., 2019; Dismore, 2016; Domecka, 2017; Hung \& Appleton, 2016). Instead, my participants had more in common with Bovill's (2012) workbased students who were able to employ reflexivity and remain "knowledgeably embedded" within their social contexts (p. 687). In contradiction to King's analogy of Archer's reflexive individual wandering alone in the world (King, 2010) (see Chapter 2.5), these distance learning students were firmly connected to the wider world.

\section{Contextual continuity, discontinuity and incongruity}

Contextual continuity, discontinuity and incongruity as defined by Archer $(2003,2007)$ offered reasonable explanations of the cohesion or breaks between an individual's natal context and their values. In contrast to her earlier studies, my study found the impacts on participants' reflexivity are not as simple or linear as Archer suggested. Deedee's position indicated contextual discongruity (and incongruity with her parent) was multi-faceted: in relationships within her adopted culture, in her citizenship status, in her hopes for her career, and in her environmental concerns, values and projects. The self as a reflexive project was enormous. Yet, Jem had a more self-contained and aligned project for her future, through shaping her contexts to fit her values and goals. Deedee's reflexivity was similar to that found in other studies (Hung \& Appleton, 2016; Matthews, 2017) of individuals who are voluntarily or involuntarily displaced in society. As people find their place in society and recognise the potential within their circumstances then the total context of their lives becomes more important than one part of it. However, knowledge of contextual stability, instability and breaks in sequence are important when considering individuals' reflexive projects, possibilities for action, and whether or not as agents they are able to flourish in a changing world.

\subsubsection{The future self}

Consideration of possibilities for the future self or a modus vivendi seems to be an important part of agency and identity. A "possible selves perspective is congruent with a narrative understanding of identity in which the self is understood as an unfolding story rather than a static state" (Rossiter, 2007, p. 92). This view of the self as static or continuously developing 
appears to be critical in a person's ability to act on knowledge and values. Kane continued to demonstrate the unfolding of his identity from his earlier upbringing, in his current social action in public service and change in direction in study and hopes for the future:

I admit I'd love to have a job at the UN one day even though that's a very vague statement because I don't even know half the crap that they do. But, just the general concept of working for an organisation with worldwide reach and involvement would just be amazing, dealing with people as people (Kane300).

In turn, it was reflected in his thinking about others and the potential for lives in an open communal world:

If an Indian family moved to New Zealand today and said they wanted to embrace what the culture is and wanted to call this place their home, I don't think they should have any less rights than any other person who is living here currently whether they are European or Māori (Kane200).

All 31 participants in the citizenship courses expressed hopes for or visualised themselves in a better world. Some were able to see how they could contribute meaningfully to this goal, while others struggled. Study in higher education should provide the opportunity for citizens to realise this improved society. In the current performative environment (Macfarlane, 2017), to achieve at an advanced level in higher education a student needs to have some autonomous reflexive characteristics in resolutely pursuing their goals. The idealism and focus on societal improvement of meta-reflexives is unlikely to see fruition within the current academic environment. However, the development of tools to recognise the often invisible graduate attributes of citizenship (for example Kensington-Miller et al. (2018)), generated as a result of reflexive consideration in a course of study, extends the possibilities for universities to encourage the growth of personal agency that is necessary to build societies in times of rapid change.

\subsubsection{Possibilities for action}

Context and considerations of the future provide the basis for consideration of the possibilities for acting on one's values and beliefs and hence possibilities for agency. Socioeconomic and cultural factors frame decision-making and shape possibilities for implementing projects for action regardless of the existing mode or modes of reflexivity. Agency exists in 
the transformation of concerns into projects [and] represents the transition from desires and priorities of a more abstract nature regarding what individuals want to be and do, to the precise definition of life goals and ways to accomplish them (Archer, 2007, p. 4).

Concerns become projects dependent on the subject's life contexts. Their contexts are where they define their priorities and goals, identify what resources they need, and the strategies required to achieving their goals. They then look at the options that best meet what they want to have, do and be (Caetano, 2019). Kahn argues that following one combination of priorities and actions can lead to "more profound and far-reaching emancipation" (2017, p. 377) than might result had a different priority and project been followed.

In this study, people were very aware of what was inside, outside or marginal in the landscape of their lives. First they decided if something is a possibility, then, as Dyke et al. (2012) found, it might become their project, or one of their projects. Following this, the participants developed a more detailed plan of what must happen for their project to become a possibility. Karen's openness to ideas and alternatives reflected her security in her identity as a family member and contributor to the social functioning of her community, and the possibilities before her. She was able to use her financial security to pursue her mediumterm project in her studies as a stepping-stone towards her longer goal - helping to improve society. Shazz was also open to new ideas and thinking and, like Karen, these were based on her community and family-based values. Both used their personal and economic resources to consider and form projects to ensure that they contributed to wider social goals. Despite this, the turn in Shazz's personal circumstances, lack of time and finances meant she let go of her longer-term community project to deal with what was a higher priority and, hopefully, shorter-term personal project. She recognised what was possible within her physical, financial and emotional resources. This finding supports the idea that possibilities for action may be directed towards individual benefit and/or communal benefit (Caetano, 2019; Mrozowicki, 2011), dependent on the resources available under the present conditions. 


\section{Enablements and constraints}

Structural enablements and constraints of possibilities for action must be worked with or around if projects are to progress. An interesting finding in my study of nine participants total stories over time (Chapter 5.1) was that barriers to action were considered in five ways:

1. as a minor complication that could be manoeuvred around, as demonstrated by Sage in driving people to the polling booth to vote;

2. flexible meaning the individual must seek to find a way through, as Nan did in looking for financial support for her community projects;

3. fixed but temporary, therefore the person could still plan an action while waiting for circumstances to change, as Deedee viewed the limitations on her circumstances while planning for employment in her new country; or

4. barriers that are considered fixed totally impeding action, as Nell's mental health condition had become; and

5. the view that barriers don't really exist, as reflected in Kane's boldness in stepping out on a new career direction.

Archer (2007) defined three steps in internal conversation - identifying concerns, deliberations in ranking concerns and working these deliberations into the modus vivendi. It is this reflexive deliberation which drives the movement through these three stages allowing scope for one's attitude to affect all these stages of agency. My study shows, contrary to expectations, that attitudes to barriers can remain fixed and can hinder agency at any step within this internal conversation, stopping reflexive progress until conditions or attitudes change.

Having the right resources aided individuals' consideration of choices and helped to decide on actions, enhancing agency. People with meta-reflexive characteristics want to act on their values and beliefs and express frustration or disappointment when projects cannot be formed or completed (Archer, 2007). Kane had the resources he needed, acted and completed a moderate-sized personal project during this study. Sage had big dreams and could see how she might help improve her own and others' conditions but lacked the social and financial resources to do so. Consequently, the emotional aspects of her reflexivity (similar to those discussed by Holmes, 2010) frustration and disappointment in her ability to act - remained with her. 
Constraints and enablements, and the attitude a person had toward structural restrictions and empowerments, appeared to determine the approach taken and the outcome towards projects. While Dyke et al. (2012) found individuals in their study "adopted" (p. 846) different reflexive approaches over short periods of time in response to their changing circumstances while developing their career project, the participants in my study did not adopt such a reactive reflexive approach. Instead, it seems the changes in situation necessitated different, more considered approaches. Further investigation of the nature and temporal effects of barriers and the immediacy or delay in reflexive responses, from existing and new biographical studies, would be beneficial in advancing understandings of these effects.

\section{Importance of self-knowledge}

Agency is dependent on agents having self-knowledge and awareness of their values, concerns and the capability or personal emergent powers to consider their self in relation to both the visible and invisible structures they encounter in daily life. People have a sense of knowing what structures exist and how they work. Knowledge of structures can be implicit or explicit; embodied as habitus, consciously and unconsciously held (Bourdieu, 1984) or in habits (Archer, 2012). Despite Archer's premise that the rapid pace of change means habitus cannot be formed, habitus remained current in participants' thinking about their social roles. Shazz accepted that within her extended family and its cultural heritage, her role as a woman meant she had limited opportunities to voice her concerns. In this context, her personal emergent powers were constrained by social structures. However, those same cultural restraints do not limit her voice and agency in another context where she was a member of a community governance board. Similarly, Dismore (2016) found part-time workplace students typically demonstrated morphogenetic processes in accepting the limitations of one situation and moving on to act in different agentic situations. Sage, meanwhile, felt she had little personal power over her situation or possibilities as her on-going financial restrictions limited her capabilities for action. Unlike Nan who had similar financial limitations, the restrictions on Sage's agency were located within her thinking. However, the ability to reflexively adapt to circumstances might be context and confidence dependent as Matthews (2017) found in international students' ability to form projects in new study environments.

\subsubsection{Project choice and implementation}

The development of personal projects depends upon what the individual feels they are capable of doing, and their interest or motivation for achieving the project. These students 
enrolled in university as a personal project, something they have control over. However, the smaller course projects did not necessary align with personal projects. For example, Karen's student group project did not resonate with her values and concerns. While she participated and shared her opinions with her younger team-mates, she did not feel sufficiently committed to pursue a careful analysis of the steps required to implement the project. In contrast, a highly motivated Jem used the group project as an opportunity to demonstrate her capabilities on a path to gain a postgraduate position. Kane secured a scholarship to study in another culture. Shazz joined a community group and volunteered to become a board member. The selection of a project and its implementation has a lot to do with personal characteristics and approaches to barriers or opportunities (Caetano, 2019). Social conditions will spur some individuals towards greater projects while similar conditions will act to restrain others.

Individual variability in projects and their flexibility or permanency will differ between people in similar contexts. Caetano (2019) found some people attempted to pursue projects that were incompatible with their lives and concerns. Deedee discussed difficulties she had in maintaining her environmental values in a new culture: planning to use only natural products with her child she found the few products that were available were well outside her budget. Projects can also change over time to match changes in the individual's concerns, for example Elsie's new project to find paid employment in the local community rather than just volunteering as a social good. Projects can be reworked and adapted as circumstance change (Caetano, 2019). They move from small-scale, short-term activities to have a larger or more permanent nature, as time and circumstance change. A person's concerns may change or need to adapt to other conditions. In stable contexts, habits and acceptance of 'what is' can limit vision, thus projects are not solely reliant on agential abilities and reflexive competencies (Caetano, 2019).

\section{Completion of projects}

Just because an individual pursues a project, it does not mean it will be successful. It depends on multiple factors: the type and timing of project - whether in the near future (hours, days, weeks, months), 'day-to-day', larger projects (Caetano, 2019), or 'future projects' (Hung \& Appleton, 2016). The closer in time to implementation the more likely preparations and plans are to be more complete and therefore the likelihood of completion increases. The type of projects considered by participants in my study ranged. Karen was half-way through 
a large career project, whereas Kane's new career project was being developed and first steps were being taken. Sage had a one-off, small project at the start of the study in getting her family members to vote. Over the period of the study, Deedee and Nan worked through a number of small one-off projects.

Projects which are further away, more complex, require more planning and preparation, or have implications for lives may not be completed (Caetano, 2019). Shazz's longer-term project had been interrupted by the change in her personal situation; other than completing their studies, Elsie and Jem had no immediate projects but long term, future projects. Projects must align with values, concerns and personal priorities and depend on the presence or absence of appropriate resources (Matthews, 2017), and other contextual constraints (Archer, 2007). The present findings suggest that using the completion, or noncompletion of projects within a set time period to determine reflexive modes and agency must be done with caution. Agency can exist in attitudes towards changes in circumstance (in the form of rejection, acceptance and resistance as discussed in Chapter 6.1) independently of project formation.

\subsection{Chapter summary}

In this chapter I discussed my study findings on higher education students' reflexive deliberations of their personal values and concerns when challenged within a course of study. I drew on the literature from chapter 2 and findings from other studies. I discussed how different approaches in reflexivity and internal conversation demonstrated how diverse students navigated the constraints and enablements of structures in their own lives and wider social networks. I elaborated on how this affected their agency to work towards a modus vivendi.

I found Archer's methodology for understanding the enablements and constraints in the structure-agency relationship, and her reflexivity theory, valuable additions to theoretical approaches used to understand the impact of higher education on transformation at personal and social levels, with modifications. However, if the goal of reflexivity is solely to create a modus vivendi (Archer, 2012, p. 223) then it is likely to fail. My findings show that reflexivity is open to revision and adjustment as new situations and combinations of factors are encountered. The new majority of higher education students have varied work-study-life roles, any combination of which may be presented in different circumstances. This reflects 
the complexity and changing contextual conditions which occur in everyday life and study for distance learning students in higher education.

Archer's use of contextual continuity and discontinuity from natal contexts did not resonate well with this group of diverse higher education students studying within the Aotearoa/New Zealand's bicultural education system in an increasingly multi-cultural population. The mobility of these students indicated natal context was part of but not a determinant of their reflexive approaches. Contrary to Archer's expectations of technological and global change separating individuals from their natal context, or those important to them, this study found participants use of technological advances and social media allowed them to retain connection to those important to them and to extend their networks.

Changes in personal circumstances do affect modus vivendi and reflexivity. Two individuals limited their reflexive processing based on their situations or changes in priorities. Others demonstrated reflexive deliberation increased or decreased as their circumstances changed. Of particular interest was the response of mothers in limiting wider reflexivity to deal with immediate family events, only to return to prior levels of reflexivity when circumstances changed. Also, of interest was the social acceptance of mental health as a part of a person's identity permitted the individual to increase their reflexive thought processes and empowered them to act.

Contrary to Archer's proposal of a dominant mode of reflexivity developed and sustained over time in association with increased education, this study did not support this idea. It concurs with other researchers that dual or multiple modes of reflexivity can exist as circumstances and opportunities for reflexivity vary. Despite the higher education culture promoting individualistic characteristics, the majority of students in this study have communal values or interests, and an interest in overall societal improvement rather than merely personal development.

Examination of values and concerns through study in a set of citizenship courses which challenged existing beliefs, produced some highly reflexive deliberations and immediate action. It also encouraged consideration of possibilities for the future self and the kind of society individuals hoped to participate in as graduates.

Finally, I presented my preliminary thinking on higher education students' reflexivity. The discussion highlighted the influence of structures on individual reality and the important of context, change and disruption on reflexive processes. I suggested that imagining the future 
self was important in pursuing a course of action or project. My study also recognised that variability in projects occurred across participants and across time and was not only tied to beliefs but to personal commitments at points in time.

Other aspects of the contribution of this research project, limitations of this study and future developments are presented in the conclusion chapter. 


\section{Chapter 7. Conclusion}

In this chapter I draw on the findings presented in Chapters 4 and 5, and discussion from Chapter 6, to identify the contribution my research project makes to existing work on reflexivity and internal conversation. I acknowledge what this research brings to build understanding of the reflexive processes of diverse higher education students as they consider value-laden knowledge in light of their personal realities. This chapter contains both my reflections and research reflexivity. I suggest more research needs to be done to understand reflexivity's place in the development of social knowledge, and in the invisible graduate skills and attitudes in the lived realities and experiences of diverse student learners. Kahn (2017) believes university study should be a place in which learners can flourish, to develop as members of society. Following this, I acknowledge the limitations of my study in the application of Archer's reflexivity methodology to develop understandings of higher education students' reflexive processes during study. I suggest further possibilities for research in light of my study's findings.

\subsection{Evolution of my research project}

My research project looked at how a group of diverse higher education students reflexively made sense of value-laden knowledge of citizenship and its place in their lives, when their personal values and concerns were challenged. The diversity within the 31 participants in this study is representative of the 'new majority' of university students who engage in lifelong learning (Felten \& Lambert, In press) to develop and maintain the employment and life skills considered necessary for living in the twenty-first century. The current emphasis within higher education is on the development of "visible" employability skills rather than the "invisible" social skills that allow members of society to operate together (Kensington-Miller et al., 2018; Macfarlane, 2017). A difficulty then for policy makers, institutions and teachers, is how to recognise and acknowledge the development of invisible social attributes. The findings from this longitudinal qualitative research suggests further consideration of students' 
reflexive focus on their own development should be included when assessing invisible attributes.

The introduction of citizenship courses in higher education is a postmodern political and cultural response to the perceived widening gap between traditional structures and the disruptions and unknowns of the globalised social world (Ahier et al., 2003; Barnett, 2009; Klemenčič, 2018). In looking at a group of diverse students' participation in a set of citizenship courses in Aotearoa/New Zealand, I wanted to understand the 'invisible', in how these students made sense of knowledge and views that differed to their own. I wanted to see if they felt that they could act on their new understandings, and if their social contexts allowed them to do so. To observe this, I understood that I needed some means of stepping inside their minds to understand their circumstances and thinking.

My theoretical approach recognised that reflexive processing takes part as an internal conversation about the structure and agential enablements and constraints of reality, as described by Archer (2003). Such internal conversation is informed by socially constructed knowledge of what reality is (Berger \& Luckmann, 1966) and could be. Critical realist ontology acknowledges that the natural, practical and social orders of reality exist outside of the person, and knowledge of that reality exists within the person (Archer, 2000). Therefore, to understand how ideas are reflexively processed by people, I considered the contribution of their wider social contexts and how individuals used their external interactions to assist their reflexivity. Archer's reflexivity methodology (which separates an individual's considerations of structures from their actions, (Archer, 2007, 2012)) allowed me to observe the inner deliberations of the participants in my study project. Modifications and adaptions in methods used in other studies (including Caetano, 2017b; Dyke et al., 2012) suggested that the collection of multiple sources of data would help to triangulate findings on an individual's reflexive processing of ideas.

The research question I wanted to answer was: How do diverse higher education students' reflexively process and act on value-laden knowledge acquired through their participation in three citizenship courses? My sub-questions to answer this question considered the knowledge, values and concerns important to these individuals, how social interactions assisted their thinking, the nature of their internal and external conversations, and how free they felt to act on their reflexive processing. 
In Chapter 2, I argued that diverse university students already have rich lives and experiences formed in primary and on-going secondary socialisation processes. These experiences and conditions informed their sense of identity and the beliefs they hold about their self and society. Their values in life, concerns and priorities in the immediate and goals for the future are integrated into their being. Critical realist Archer and structuralist Giddens thought that a rapidly changing world would lead to a more reflexive society - a society that reified the self as separate from traditional structures and inherited culture. Their concerns were that people were too embedded in their existing social structures and would not be able to cope in a changing world unless they developed higher reflexive capabilities (Archer, 2000; Giddens, 1994). Archer proposed a typology of reflexivity of the forms of individuals' reflexive processing she expected to see. She tested these on specific social groups and observed certain mental activities were associated with the individuals who fit her reflexivity modes (Archer, 2003). These mental activities were used to see how individuals fit Archer's modes of reflexivity (Archer, 2007). Archer believed her model portrayed a progression from less to more sophisticated reflexive processes. She considered study in higher education as a place where individuals develop higher order thinking and the characteristics of metareflexivity. Therefore, students entering a period of study of an undergraduate degree should develop meta-reflexivity over time.

Archer's work on progression in reflexivity through education raised questions for me: what reflexive characteristics do diverse students in Aotearoa/New Zealand higher education studying citizenship knowledge and values demonstrate? Do they fit Archer's reflexivity modes or are there other factors at play? And do they feel that they can act on new understandings from their studies?

In Chapter 3, I justified my novel use of qualitative longitudinal research methodology (QLR), embedded case study design and narrative methods to answer these questions. The setting up of the case study, recruitment and retention of 31 distance learning participants enrolled in three citizenship courses proved challenging. Showing manaakitanga, a culturally and socially responsible approach to research in Aotearoa/New Zealand, fostered the researcher-participant relationship and allowed for the collection of a rich set of data. The novel use of framework analysis (Lewis, 2007; J. Smith \& Firth, 2011) as a sole researcher allowed me to look for themes at both points in time and changes across time and to retain participant stories. The core citizenship courses and activities intentionally challenged 
students' beliefs and values and encouraged students to think about the knowledge presented in terms of a wider range of peoples' beliefs and experiences.

Broader findings from the embedded case study in Chapter 4, showed how encountering new citizenship ideas, values and beliefs had challenged participants' thinking at a deeper level. One third of students described on-going, deep reflective thinking, questioning and testing ideas; allowing ideas to jostle within life demands. In this thinking, the presented concepts were compared with the learners' own life experiences including, but not exclusively, their natal contexts. Half of the students felt it was important to talk to close family members and friends to gather other ideas, to build their own resources or to test their thinking against other ideas. These actions led to further reflexive activities and empowerment.

This finding led me to consider using Archer's internal conversation and reflexivity typology as the method of analysis. In subsequent interviews, I asked participants whether they used Archer's ten mental activities and how. I then looked for confirmatory evidence of these activities from their interviews and written data. I compared participants' reflexivity data to see if there was a fit with Archer's descriptions of her four reflexivity modes (Archer, 2003, 2007, 2012).

In Chapters 4 and 5, I presented my findings on the social encounters diverse university students drew on to inform, test or spread their thoughts based on their interview and written data. I recognised how they built on their existing knowledge of social activities to dream about actions which they believed would improve their social world. I analysed their agency in terms of their lived realities and changing circumstances, and the effects these changes had on their actions, dreams and desires. Participants' narratives gave me a fuller picture of their lived realities, their reflexive experiences and the possibilities they had to act on their new understandings. It appears personal circumstances have a greater influence on reflexivity than signified by Archer. These findings on distance students, a subset of the higher education population, extend and contrast the existing data on students' use of reflexivity during their studies. The findings deepen and confirm the empirical work of Dyke et al. (2012), which also critiqued Archer's framework.

In Chapter 6, I discussed these findings in terms of Archer's assumption that a dominant and progressive mode of reflexivity prevailed as educational level increased. I discussed the relationship between dual, multiple and changing modes of reflexivity exhibited by my 
participants as their social and personal circumstances changed. I came to understand that the foundations for agency are not equal nor accessible for everyone. Nor did their modes of reflexivity mirror those put forward by Archer and other researchers (Caetano, 2017a; Mrozowicki, 2011). Theoretical and practical understandings of reflexivity, its modes, their traits and processes are works in progress. In Chapter 6, I also presented a conceptual framework of the layers, factors and relationships that, I believe, need further consideration as understandings of reflexivity progress.

\subsection{Contribution of my research}

My study brings new information to sociological discussions on structure-agency and how reflexive deliberations inform the actions of agents. Specifically, it considers the interaction between diverse higher education students' reflexive processing of their beliefs and values when these are challenged within their study context.

\subsubsection{Reflexivity and internal conversation theory}

In Chapter 2, I drew together a wide body of scholarly material on structure-agency, reflexivity and internal conversation theory to examine the developments, debates and gaps in understanding. This discussion will be of use to other higher education scholars with interest in the topic.

\section{Multiple modes and combinations of reflexivity}

My research indicates that diverse Aotearoa/New Zealand higher education students' reflexive practices are highly contextual and influenced by changes in personal circumstances. The specificity of reflexive processing means typologies of reflexivity need to be examined for their robustness across cultures and contexts. Globalised worlds sit within, across and beyond traditional borders; understandings of reflexivity beyond AngloSaxon and European contexts are in their infancy. In comparing my findings from this small study with Archer's modes of reflexivity, I showed that dual modes of reflexivity were present at any one time as people worked through the complexities of their work-study-life realities in Aotearoa/New Zealand, a country with a bicultural foundation and multi-cultural population. I showed that reflexive processes and therefore alignment with Archer's reflexivity modes changed over time. Multiple modes and combinations may be used across time. I suggest diverse higher education students' circumstances and experiences may be more important 
in determining reflexive typologies than Archer's and Caetano's focus on educational attainment and distance from natal contexts. This complexity adds to the higher education and sociological literature by highlighting the multiple and temporal nature of reflexivity.

\section{Controlled reflexivity}

A significant finding from this study is the use of a controlled form of reflexivity by three individuals to balance their concerns against their personal projects and goals within difficult or challenging situations. This reflects different levels of agency and adjustment to the delicacies of personal circumstances such as relationship establishment and mental health challenges. The finding on controlled reflexivity brings new understandings to reflexivity theory on the use of personal reflexivity to achieve both personal goals and emotional stability.

\section{Connection to natal contexts}

My study showed that contextual continuity, discontinuity and incongruity in the structureagency relationship is not a simple sum of individuals remaining with or stepping away from their natal contexts. It involves other aspects of priorities, social connectedness, culture, age and expectations rather than just significant events. While changes between home life and on-campus university life can have a great impact in younger lives (Baker, 2019; Barratt et al., 2019; Matthews, 2017), differences between natal and present social and study contexts is not as prominent for older, more experienced distance students. Participants demonstrated the important use of technology to retain rich connections with one's natal settings despite physical displacement. This analysis illustrates how Archer's conceptions of social disruption in late modernity were overstated and need not have the same effect in bicultural or multicultural societies.

\section{External conversations enhanced internal conversations}

Internal conversations were a part of my participants' lives. However, the intensity and nature of the inner deliberation differed and changed with personal circumstances. In keeping with Archer's definition of internal conversations, mental activities of internal conversation were used broadly to compare and to contrast ideas presented in academic study and daily life. They were used in a more focused manner to examine ideas that were challenging personal values or were of a complex nature. To understand complex ideas, 
most participants also used external conversations to build their knowledge bank. They then considered this new knowledge in the privacy of their internal conversations. To be reflexive about citizenship and to act on these inner deliberations required an individual to have a good understanding of their identity, values, concerns or priorities.

Internal conversation and the mental activities of reflexivity were the primary data that Archer used to determine her modes of reflexivity. However, my study showed that external conversation also built individuals' internal conversations through supplying alternative viewpoints and additional resources to inform thinking. Writing activities complemented or structured thinking, which aided reflexive thinking. My study supports Caetano (2017b) and Chalari's (2007) work on gathering wider evidence of reflexive processing in external conversations and reflective writing, rather than relying solely on interviews. However, I believe external conversation and written data sources need to be used carefully.

\section{Community focus reflexivity produces action}

The focus of my participants' reflexive processing of concerns at individual level or community level was not associated with one reflexive mode. My study supports Mrozowicki's (2011) elaboration on this characteristic of reflexivity for societies under change. An individual's focus on wider community concerns, or dreams for wider society, appeared to generate more creative thinking of the possibilities for action. It affected the scale, timing and types of projects considered. Community-focused reflexivity appears to produce more positive and firmer actions, and to build agency. Small projects focused on others were selected and implemented within the period of this study. Further evidence of reflexivity's focus on the self or others may be found in future analysis of data from the remainder of my original participant group or in other studies.

These findings extend our understanding of reflexivity theory and its application in social and cultural contexts in younger, non-traditional countries where the impacts of late modernisation are moderated in the presence of societal diversity.

\subsubsection{Higher education students' development of invisible skills}

Throughout my research I present Aotearoa/New Zealand's higher education context, like UK, Portugal, Poland and any other cultural grouping, as a unique setting. Aotearoa/New Zealand's recent history, geographic location and bicultural focus generates its own set of beliefs and circumstances which influence reflexive views of self and others. The findings 
from this study are representative of the current cultural, social and temporal contexts of this specific group of diverse Aotearoa/New Zealand students. This was evident in the reflexive experiences of the two participants living in other countries during this inquiry. They needed to translate their values and priorities between different cultural contexts in their reflexive deliberations. However, the implications from the findings in this study have relevance to the development of wider understandings of reflexivity and reflexive processes in other groups. The ability to reflexively process different values and beliefs and adjust one's thoughts on reality to accommodate others' viewpoints is an invisible skill all university students should develop over time. How this characteristic is recognised and assessed in university graduate profiles and attributes needs to be considered by universities and teachers.

The case study of a set of citizenship courses intentionally designed to challenge students' existing ideas and values on citizenship and its structures, provided a useful context for examining reflexivity and approaches to assessments. Having been challenged, students' recognition of personal and social identities produced a layering of reflexive thinking on their personal priorities and social concerns. Students' primary concerns and reflexive positions matched those described in Archer's reflexivity typology. Students with meta-reflexive characteristics focused on moral concerns and issues, looking for solutions to problems. However, in this study, while autonomous reflexives' primary concerns were their selffocused goals and solution seeking strategies, they did not lack social consciousness. They saw their current commitments as part of a long-term solution to social issues. This reflexive commitment to long term societal good is currently under-valued as a defining attribute of university study and graduate students. It needs to be elevated.

Understanding that reflexive processes appear to be managed by some individuals to suit their particular work-study-lives situations, means current evaluations looking only at visible measures of university student engagement will likely miss the complex realities of a student's study world. Of particular interest from the findings of my study were the impacts that changes within family groups, in living conditions, or societal recognition of roles, had on the reflexive processes of students. In particular, those who were parents or dealing with mental health difficulties did not have the same reflexive focus on their studies when they were coping with the wider realities of their lives. At a practical level, their ability to join and contribute to group work was severely restricted by lack of understanding of their circumstances. Reflexivity and agency should not be inextricably bound together as it is possible to be reflexive but not agentic and to demonstrate agency without reflexivity. 
Recognising such constraints is important when gathering evidence of students' development of the visible and invisible skills and attitudes.

This research on reflexivity when values and beliefs were intentionally challenged contributes to conversations on the manner in which teachers can recognise and evaluate personal growth and development as an outcome of university study. Not all student development may be assessed by traditional means. While the visible skills and attributes are the focus of the "performative turn" (Macfarlane, 2017) of higher education, current assessment practices continue to compromise understandings of students' social development and their agency to contribute to social progress. Providing opportunities for learners to recognise and understand growth in their own reflexive practice (the invisible graduate capabilities) over a consecutive period of study would be an enhancement on the reflective exercises currently used in assessments.

\subsection{Limitations of my study}

There are a number of study limitations that need to be acknowledged in reflecting on the outcomes of the project. The findings from my study represent the reflexive deliberations of a group of self-selected students with an interest in participating in this research study. This may have biased the study in two ways. First, over half were seeking to push-back against an imposed structure in being required to take the citizenship courses. The opportunity to take part in the study may have generated particular reflexive thoughts. Second, the giving of vouchers as a koha/gift also touched on a primary concern - having enough resources to get by on. While the koha/gift was small, it was seen as significant, which may have resulted in some participants framing their responses in return as a koha/gift.

I do not present any typified modes of reflexivity and internal conversation for this small group of diverse higher education students. The variability in their contexts, nature and use of reflexive processing means general representation is not possible with such a small number.

This study gathered some biographical data, but only what was offered by participants. Other studies have drawn on extended or multiple, open conversational interviews (Caetano, 2015b; Mrozowicki, 2011) or interviews with extended networks (Dyke et al., 2012; Matthews, 2017) to gather in-depth biographical information. The semi-structured interview techniques used in my research were flexible enough to allow some extended conversations. However, 
the time constraints on my research project and the participants' busy lives limited the possibilities for longer or divergent discussions that may have revealed more personal information relevant to their reflexive processes.

\subsection{Future directions for research}

The exploratory nature of this research and reflexivity theory raises more questions and additional possibilities for further investigation. Underlying social theory is the assumption that life is lived out in all three orders - the natural, the practical and the social. Yet, circumstances change which individuals have no control over. Social limitations restrict choices, and the demands of life roles and priorities affects reflexive activities and outcomes.

Archer's concerns that societal change and technological advancements would cut people off from family connections in modern industrialised societies, requiring them to use higher orders of reflexive processing, still requires further exploration. There is no doubt that the rapid pace of globalisation and its effects are changing the natural, social and practical orders. Social disruption means the predictable, predetermined paths of social and cultural interactions have changed. Technology has altered the way individuals interact with each other, and the structures they encounter. Constraints on privacy and the use of technology now require individuals to sometimes interact with systems on their own, rather than always drawing on the collective knowledge, values and support of their social groups. Further investigation needs to ask: how have technological advancements in access to the internet and social media contributed to an individual's understanding of the structure-agency relationship and their social connectedness or social separation?

The presence of multiple modes of reflexive characteristics in this small but diverse group leaves me to ask - how representative is this finding of different or similar higher education student groups? Likewise, is the change in reflexive processing when life routines are disrupted typical of those higher education students who are parents or deal with mental health issues, or a general characteristic of the wider population? The appearance of controlled reflexivity and underlying communal or individual foci of reflexive processing within Aotearoa/New Zealand bicultural and multicultural environment also requires further investigation. Further analysis of the data from the remainder of the original participant group may provide more information towards answering these questions. 
Different approaches in considering reflexivity and the use of internal conversation, external dialogue and writing have demonstrated how diverse university students navigate the constraints and enablements of structures within their own lives, and the intersections of their lives with wider social networks. It is through the use of social network processes that fully informed reflexivity allows a person to understand their agency and how much freedom they do have to work towards a modus vivendi. There is considerable potential in developing a reflexivity tool for self-assessment that would extend higher education learners' understandings of their own agency and possibilities for social action across multiple facets of twenty-first century society.

\subsection{Final words}

Higher education's purpose is not solely to build the economic prosperity of society. Most people will only spend a third of each day in employment and most of their jobs will involve social interaction with others. All people need to know how to engage with others and to work towards collective goals, despite individual differences in beliefs and goals. As my study demonstrates, this can only happen if people are prepared to consider what is important to others.

Archer's consideration of reflexivity and understandings of agency offers a way to understand what happens when higher education students encounter other people, and values and ideals that differ from their own. Global uncertainty and change should not restrict individuals' growth; rather it should enhance their meaningful contribution to society. If higher education continues to provide an environment that emphasises autonomy and personal gain over societal improvement, then diverse learners with communicative and metareflexive ways of thinking are likely to be frustrated by the lack of opportunities to contribute meaningfully to societal change. 
If we believe our knowledge, values and beliefs are socially constructed, then we need some means of recognising the social braids which inform that construction.

If we recognise our diverse higher education student cohorts bring with them bodies of experience and cultures which inform and define them, then we need some means of understanding how new knowledge intersects with their lived experience.

If we want our learners to rise beyond the teaching and learning interaction to develop the skills and capabilities that build societies, then we need to recognise the mastery they already have and the ways in which they contribute to society.

\section{Equally so,}

if we want people to grow as citizens through their higher education then we need to know what social circumstances advance this cause or hold them back from meaningful participation.

(Linda Rowan, Research Journal 2019) 


\section{References}

Adams, M. (2003). The reflexive self and culture: A critique. British Journal of Sociology, 54(2), 221-238. doi:https://doi-org/10.1080/0007131032000080212

Adamson, L., \& Chojenta, C. (2007). Developing relationships and retaining participants in a longitudinal study. International Journal of Multiple Research Approaches, 1(2), 137-146. doi:https://doi.org/10.5172/mra.455.1.2.137

Adamson, L., Young, A., \& Byles, J. E. (2007). Recruiting for a longitudinal study: Who to choose, how to choose and how to enhance participation? International Journal of Multiple Research Approaches, 1(2), 126-136. doi:https://doi.org/10.5172/mra.455.1.2.126

Ahier, J., Beck, J., \& Moore, R. (2003). Graduate citizens? Issues of citizenship and higher education. London, UK and New York, NY: RoutledgeFalmer.

Akram, S., \& Hogan, A. (2015). On reflexivity and the conduct of the self in everyday life: Reflections on Bourdieu and Archer. The British Journal of Sociology, 66(4), 605624. doi:https://doi.org/10.1111/1468-4446.12150

Archer, M. S. (2000). Being human: The problem of agency. Cambridge, UK: Cambridge University.

Archer, M. S. (2003). Structure, agency and the internal conversation. Cambridge, UK: Cambridge University.

Archer, M. S. (2007). Making our way through the world: Human reflexivity and social mobility. Cambridge, UK: Cambridge University.

Archer, M. S. (2008). The internal conversation: Mediating between structure and agency: Full research report ESRC End of Award report, RES-000-23-0349. Retrieved from Swindon, UK:

Archer, M. S. (2010a). Routine, reflexivity, and realism. Sociological Theory, 28(3), 272-303. doi:https://doi.org/10.1111/i.1467-9558.2010.01375.x

Archer, M. S. (2012). The reflexive imperative in late modernity. Cambridge, UK: Cambridge University.

Archer, M. S. (2013). Collective reflexivity: A relational case for it. In C. Powell \& F. Dépelteau (Eds.), Conceptualizing relational sociology: Ontological and theoretical issues (pp. 145-161). New York, USA: Palgrave Macmillan.

Archer, M. S. (Ed.) (2010b). Conversations about reflexivity. New York, NY: Routledge.

Archer, M. S., Bhaskar, R., Collier, A., Lawson, T., \& Norrie, A. (Eds.). (1998). Critical realism: Essential readings. London, UK and New York, NY: Routledge.

Archer, M. S., Decoteau, C., Gorski, P., Little, D., Porpora, D., Rutzou, T., . . . Vandenberghe, F. (2016). "What is Critical Realism?". Perspectives, 38(2), 4-9.

Arthur, J., \& Bohlin, K. E. (2004). Citizenship and higher education: The role of universities in communities and society. New York, NY: RoutledgeFalmer.

Ashwin, P. (2008). Accounting for structure and agency in 'close-up' research on teaching, learning and assessment in higher education. International Journal of Educational Research, 47(3), 151-158. doi:https://doi.org/10.1016/i.ijer.2008.01.002

Ashwin, P. (2014). Knowledge, curriculum and student understanding in higher education. Higher Education, 67, 123-126. doi:https://doi.org/10.1007/s10734-014-9715-3 
Austin-Wells, V., McDougall, G. J., \& Becker, H. (2006). Recruiting and retaining an ethnically diverse sample of older adults in a longitudinal intervention study. $\begin{array}{lll}\text { Educational } \quad \text { Gerontology, } & \text { 32(2), }\end{array}$ doi:https://doi.org/10.1080/03601270500388190

Australian Government Ministry of Education. (2018). Selected higher education statistics 2018 Student data. Retrieved from https://www.education.gov.au/selected-highereducation-statistics-2018-student-data

Baker, Z. (2019). Reflexivity, structure and agency: Using reflexivity to understand further education students' higher education decision-making and choices. British Journal of Sociology of Education, $40(1), \quad$ 1-16. doi:https://doi.org/10.1080/01425692.2018.1483820

Barnes, N. (2017). Navigating social integration into university on Facebook: Insights from a longitudinal study. Student Success, 8(1), 1-11. doi:https://doi.org/10.5204/ssj.v8i1.362

Barnett, R. (2009). Knowing and becoming in the higher education curriculum. Studies in Higher Education, 34(4), 429-440. doi:10.1080/03075070902771978

Barratt, C., Appleton, P., \& Pearson, M. (2019). Exploring internal conversations to understand the experience of young adults transitioning out of care. Journal of Youth Studies, 1-17. doi:https://doi.org/10.1080/13676261.2019.1645310

Barrie, S. C. (2006). Understanding what we mean by the generic attributes of graduates. Higher Education, 51(2), 215-241. doi:https://doi.org//10.1007/s10734-004-6384-7

Bauman, Z., \& Vecchi, B. (2004). Identity. Cambridge, UK: Polity.

Bazeley, P. (2013). Qualitative data analysis: Practical strategies. London, UK: Sage.

Beck, U. (1994). The reinvention of politics: Towards a theory of reflexive modernization. In U. Beck, A. Giddens, \& S. Lash (Eds.), Reflexive modernization: Politics, tradition and aesthetics in the modern social order (pp. 1-55). Cambridge, UK: Polity.

Beck, U., Bonss, W., \& Lau, C. (2003). The theory of reflexive modernization: Problematic, hypothesis and research programme. Theory, Culture \& Society, 20(2), 1-33. doi:https://doi.org/10.1177/0263276403020002001

Beck, U., Giddens, A., \& Lash, S. (1994). Reflexive modernization: Politics, tradition and aesthetics in the modern social order. Cambridge, UK: Polity.

Belich, J. (2007). Globalization and the nation. Paper presented at the Concepts of the Nation Symposium, Wellington, New Zealand. https://nzhistory.govt.nz/files/documents/JamesBelich-GlobalizationandNation.pdf

Berger, P. L., \& Kellner, H. (1981). Reinterpreted: An essay on method and vocation. Garden City, NY: Anchor/Doubleday.

Berger, P. L., \& Luckmann, T. (1966). The social construction of reality: A treatise in the sociology of knowledge. New York, USA: Penguin.

Berger, P. L., \& Pullberg, S. (1965). Reification and the sociological critique of consciousness. History and Theory, 4(2), 196-211. doi:https://doi.org/10.2307/2504151

Bhaskar, R. (2014). An introduction to critical realism: Part one transcendental realism.

Biesta, G., Bouverne-De Bie, M., \& Wildemeersch, D. (2014). Civic learning, democratic citizenship and the public sphere. [electronic resource]: Dordrecht : Springer, [2014].

Biesta, G., \& Simons, M. (2009). Higher education and European citizenship as a matter of public concern. European Educational Research Journal, 8(2), 142 - 1455. doi:https://doi.org/10.2304/eeri.2009.8.2.142

Biesta, G., \& Tedder, M. (2007). Agency and learning in the lifecourse: Towards an ecological perspective. Studies in the Education of Adults, 39(2), 132-149. doi:https://doi.org/10.1080/02660830.2007.11661545 
Bourdieu, P. (1984). Distinction: A social critique of the judgement of taste (translated by Richard Nice). Cambridge, MA: Harvard University.

Bourdieu, P. (1998). Practical reason. Cambridge, UK: Polity with Blackwell.

Bourdieu, P., \& Wacquant, L. J. D. (1992). The practice of reflexive sociology (The Paris Workshop). In An invitation to reflexive sociology (pp. 216-260). Chicago, IL: University of Chicago.

Bovill, H. (2012). The importance of internal conversations and reflexivity for work-based students in higher education: Valuing contextual continuity and 'giving something back'. International Journal of Lifelong Education, 31(6), 687 - 703. doi:https://doi.org/10.1080/02601370.2012.723049

Brown, M., Hughes, H., Keppell, M., Hard, N., \& Smith, L. (2015). Stories from students in their first semester of distance learning. International Review of Research in Open and Distributed Learning, 16(4), 1-17. doi:https://doi.org/10.19173/irrodl.v16i4.1647

Buissink-Smith, N., Mann, S., \& Shephard, K. (2011). How do we measure affective learning in higher education? Journal of Education for Sustainable Development, 5(1), 101114. doi:https://doi.org//10.1177/097340821000500113

Buissink, N., Diamond, P., Hallas, J., Swann, J., \& Sciascia, A. D. (2017). Challenging a measured university from an indigenous perspective: Placing 'manaaki' at the heart of our professional development programme. Higher Education Research \& Development, 36(3), 569-582. doi:https://doi.org/10.1080/07294360.2017.1288706

Burkitt, I. (2010). Dialogues with self and others: Communication, miscommunication, and the dialogical unconscious. Theory \& Psychology, 20(3), 305-321. doi:https://doi.org/10.1177/0959354310362826

Burkitt, I. (2012). Emotional reflexivity: Feeling, emotion and imagination in reflexive dialogues. Sociology, 46(3), 458-472. doi:https://doi.org/10.1177/0038038511422587

Burkitt, I. (2013). Self and others in the field of perception: The role of micro-dialogue, feeling, and emotion in perception. Journal of Theoretical and Philosophical Psychology, 33(4), 267-279. doi:https://doi.org/10.1037/a0030255

Burkitt, I. (2016). Relational agency: Relational sociology, agency and interaction. European $\begin{array}{llll}\text { Journal of Social } & \text { Theory, }\end{array}$ doi:https://doi.org/10.1177/1368431015591426

Burkitt, I. (2018). Relational agency. In F. Dépelteau (Ed.), The Palgrave handbook of relational sociology (pp. 523-538). doi:https://doi.org/10.1007/978-3-319-66005926

Caetano, A. (2014). Reflexivity and social change: A critical discussion of reflexive modernization and individualization theses. Portuguese Journal of Social Science, 13(1), 93-109. doi:https://doi.org/10.1386/pjss.13.1.93 1

Caetano, A. (2015a). Defining personal reflexivity: A critical reading of Archer's approach. European Journal of Social Theory, 18(1), 60-75. doi:https://doi.org/10.1177/1368431014549684

Caetano, A. (2015b). Personal reflexivity and biography: Methodological challenges and strategies. International Journal of Social Research Methodology, 18(2), 227-242. doi:https://doi.org/10.1080/13645579.2014.885154

Caetano, A. (2017a). Coping with life: A typology of personal reflexivity. The Sociological Quarterly, 58(1), 32-50. doi:https://doi.org/10.1080/00380253.2016.1246904

Caetano, A. (2017b). Reflexive dialogues: Interaction and writing as external components of personal reflexivity. Sociological Research Online, 22(4), 66-86. doi:https://doi.org/10.1177/1360780417732956 
Caetano, A. (2018). O léxico das crises biográficas: The lexicon of biographical crises. Analise Social, 53(226), 88-111. doi:http://dx.doi.org/10.31447/AS00032573.2018226.04

Caetano, A. (2019). Designing social action: The impact of reflexivity on practice. Journal for the Theory of Social Behaviour, 49, 146-160. doi:https://doi.org/10.1111/itsb.12196

Case, J. M. (2013). Researching student learning in higher education: A social realist approach. London, UK: Routledge.

Case, J. M. (2015). A social realist perspective on student learning in higher education: the morphogenesis of agency. Higher Education Research \& Development, 34(5), 841852. doi:https://doi.org/10.1080/07294360.2015.1011095

Chalari, A. (2007). The relationship between internal and external conversation. (Doctor of Philosophy in Sociology). University of Warwick,

Chalari, A. (2013). The casual impact of resistance: Mediating between resistance and internal conversation about resistance. Journal for the Theory of Social Behaviour, 43(1), 66-86. doi:https://doi.org/10.1111/jtsb.12003

Cieslik, M. (2006). Reflexivity, learning identities and adult basic skills in the United Kingdom. British Journal of Sociology of Education, 27(2), 237-250. doi:https://doi.org/10.1080/01425690600556461

Clegg, S. (2005). Theorising the mundane: The significance of agency. International Studies in Sociology of Education, 15(2), 149-163. doi:https://doi.org/10.1080/09620210500200137

Clegg, S. (2015). Adventures in meaning making: Teaching in higher education 2005-2013. Teaching in Higher Education, 20(4), 373-387. doi:https://doi.org/10.1080/13562517.2015.1020781

Coetzee, M., \& Oosthuizen, R. M. (2012). Students' sense of coherence, study engagement and self-efficacy in relation to their study and employability satisfaction. Journal of $\begin{array}{llll}\text { Psychology in } & \text { 22(3), }\end{array}$ doi:https://doi.org/10.1080/14330237.2012.10820536

Colby, A., Ehrlich, T., \& Beaumont, E. (2003). Educating undergraduates for responsible citizenship. Change, 35(6), 40-48. doi:10.1080/00091380309604127

Cousin, G. (2009). Researching learning in higher education: An introduction to contemporary methods and approaches. New York, NY: Routledge.

Cranton, P. (2006). Understanding and promoting transformative learning $\left(2^{\text {nd }}\right.$ ed.). San Francisco: Jolly and Bass.

Cross, S., \& Markus, H. (1991). Possible selves across the life span. Human Development, 34(4), 230-255. doi:https://doi.org/10.1159/000277058

Crotty, M. (1998). The foundations of social research: Meaning and perspective in the research process. Crows Nest, NSW: Allen \& Unwin.

Cuthbert, R. (2017). The future of global HE: More (than) research is needed. Retrieved from https://srheblog.com/2017/09/15/the-future-of-global-he-more-than-researchis-needed/\#more-1104

D'Cruz, H., Gillingham, P., \& Melendez, S. (2007). Reflexivity, its meanings and relevance for social work: A critical review of the literature. British Journal of Social Work, 37, 73-90. doi:https://doi.org/10.1093/bjsw/bcl001

Denzin, N. K., \& Lincoln, Y. S. (Eds.). (2000). Handbook of qualitative research (2 ed.). USA: Sage.

Denzin, N. K., \& Lincoln, Y. S. (Eds.). (2008). Collecting and interpreting qualitative materials (3rd ed.). Thousand Oaks, CA: Sage.

Denzin, N. K., \& Lincoln, Y. S. (Eds.). (2013). The landscape of qualitative research (4th ed.). Los Angeles, CA: Sage. 
Dismore, H. (2016). Learning to learn in higher education: developing a modus vivendi. International Journal of Lifelong Education, 35(3), 254-269. doi:https://doi.org/10.1080/02601370.2016.1165746

Domecka, M. (2017). Agency, reflexivity and structure: Biographical patterns of coping and non-coping of young Italians. In A. Spanò (Ed.), I giovani del Sud di fronte alla crisi: Strategie di sopravvivenza e capacità di innovazione. Italy: FrancoAngeli.

Donati, P. (2016). The 'Relational Subject' According to a Critical Realist Relational Sociology. Journal of Critical Realism, 15(4), 352-375. doi:10.1080/14767430.2016.1166728

Donati, P., \& Archer, M. S. (2015). The relational subject. Cambridge, UK: Cambridge University.

Dyke, M. (2015). Reconceptualising learning as a form of relational reflexivity. British Journal of Sociology of Education, 36(4), 542-557. doi:https://10.1080/01425692.2013.843445

Dyke, M., Johnston, B., \& Fuller, A. (2012). Approaches to reflexivity: Navigating educational and career pathways. British Journal of Sociology of Education, 33(6), 831-848. doi:https://doi.org/10.1080/01425692.2012.686895

Elder-Vass, D. (2007). Reconciling Archer and Bourdieu in an emergentist theory of action. Sociological Theory, 25(4), 325-346. doi:https://doi.org/10.1111/i.14679558.2007.00312.x

Elder-Vass, D. (2012). The reality of social construction. Cambridge, UK and New York, NY: Cambridge University.

Ellery, K., \& Baxen, J. (2015). 'I always knew I would go to university': A social realist account of student agency. South African Journal of Higher Education, 29(1), 91-107. Retrieved from https://hdl.handle.net/10520/EJC172796

Elliott, A. (2014). Contemporary social theory: An introduction (Routledge Ed. 2 ed.). London, UK: Routledge.

Elliott, J., Holland, J., \& Thomson, R. (2012). Longitudinal and panel studies. In P. Alasuutari, L. Bickman, \& J. Brannen (Eds.), The Sage handbook of social research methods (pp. 228-248). London, UK: Sage.

Elster, J. (2017). The temporal dimension of reflexivity: Linking reflexive orientations to the stock of knowledge. Distinktion: Journal of Social Theory, 18(3), 274-293. doi:https://doi.org/10.1080/1600910X.2017.1397527

Emirbayer, M., \& Mische, A. (1998). What is agency? American Journal of Sociology, 103(4), 962-1023. doi:https://doi.org/10.1086/231294

Farrugia, D., \& Woodman, D. (2015). Ultimate concerns in late modernity: Archer, Bourdieu and reflexivity. The British Journal of Sociology, 64(4), 626-644. doi:https://doi.org/10.1111/1468-4446.12147

Felten, P., Abbot, S., Kirkwood, J., Long, A., Lubicz-Nawrocka, T., Mercer-Mapstone, L., \& Verwoord, R. (2019). Reimagining the place of students in academic development. International Journal for Academic Development, 24(2), 192-203. doi:https://doi.org/10.1080/1360144X.2019.1594235

Felten, P., \& Lambert, L. M. (In press). Relationship-rich education: How human connections drive success in college. Maryland, USA: John Hopkins Press.

Gergen, K. J. (2011). The self as social construction. Psychological Studies, 56(1), 108-116. doi:https://doi.org/10.1007/s12646-011-0066-1

Gergen, K. J. (2015). An invitation to social construction (3rd ed.). London, UK: Sage.

Giddens, A. (1984). The constitution of society: Outline of the theory of structuration. Cambridge, UK: Polity.

Giddens, A. (1991). Modernity and self-identity: Self and society in the late modern age. Stanford, CA: Stanford University. 
Giddens, A. (1994). Living in a post-traditional society. In U. Beck, A. Giddens, \& S. Lash (Eds.), Reflexive modernization: Politics, tradition and aesthetics in the modern social order (pp. 56-109). Cambridge, UK: Polity.

Giddens, A., \& Cassell, P. (1993). The Giddens reader. London, UK: Macmillan.

Giddens, A., \& Sutton, P. W. (2014). Essential concepts in sociology (2nd ed.). Malden, MA: Polity.

Gronow, A. (2008). The over- or the undersocialized conception of man? Practice theory and the problem of intersubjectivity. Sociology, 42(2), 243-259. doi:https://doi.org/10.1177/0038038507087352

Guba, E. G., \& Lincoln, Y., S. (1998). The landscape of qualitative research: Theories and issues. In N. K. Denzin, \& Lincoln, Y.S. (Ed.), Competing paradigms of qualitative research (pp. 195-220). Thousand Oaks, CA: Sage.

Guest, G., MacQueen, K. M., \& Namey, E. E. (2012). Applied thematic analysis. Thousand Oaks, CA: Sage.

Guiney, P. (2014). Extramural students' participation and achievement: Trends, patterns and highlights. Retrieved from Wellington, N: https://www.educationcounts.govt.nz/ data/assets/pdf file/0007/145744/Extramur al-Students-Participation-and-Achievement.pdf

Haggis, T. (2009). What have we been thinking of? A critical overview of 40 years of student learning research in higher education. Studies in Higher Education, 34(4), 377-390.

Harland, T., \& Pickering, N. (2011). Values in higher education teaching. New York, NY: Routledge.

Hickey, A., \& Austin, J. (2007). Pedagogies of self: Conscientising the personal to the social. International Journal of Pedagogies and Learning, 3(1), 21-29. doi:https://doi.org/10.5172/ijpl.3.1.21

Holmes, M. (2010). The emotionalization of reflexivity. Sociology, 44(1), 139-154. doi:https://doi.org/10.1177/0038038509351616

Holmes, M. (2015). Researching emotional reflexivity. Emotion Review, 7(1), 61-66. doi:https://doi.org/10.1177/1754073914544478

Hung, I., \& Appleton, P. (2016). To plan or not to plan: The internal conversations of young people leaving care. Qualitative Social Work, 15(1), 35-54. doi:https://doi.org/10.1177/1473325015577408

James, A., Taylor, B., \& Francis, K. (2014). Researching with young people as participants: Issues in recruitment. Contemporary Nurse, 47(1-2), 36-41. doi:https://doi.org/10.5172/conu.2013.4564

Jarvis, P. (2007). Globalisation, lifelong learning and the learning society: Sociological perspectives. London, UK and New York, NY: Routledge.

Josselson, R. (2013). Interviewing for qualitative inquiry. New York, USA: Guildford.

Kahn, P. E. (2013). The informal curriculum: A case study on tutor reflexivity, corporate agency and medical professionalism. Teaching in Higher Education, 18(6), 631-642. doi:https://doi.org/10.1080/13562517.2013.774356

Kahn, P. E. (2017). The flourishing and dehumanization of students in higher education. Journal of Critical Realism, 16(4), 368-382. doi:https://doi.org/10.1080/14767430.2017.1347444

Kahn, P. E., Everington, L., Kelm, K., Reid, I., \& Watkins, F. (2017). Understanding student engagement in online learning environments: the role of reflexivity. Education Technology Research Development, 65, 203-218. doi:https://doi.org/10.1007/s11423-016-9484-z

Kahu, E. R. (2013). Framing student engagement in higher education. Studies in Higher Education, 38(5), 758-773. doi:https://doi.org/10.1080/03075079.2011.598505 
Kahu, E. R., \& Gerrard, H. (2018). The use of personal experience as a strategy for critical reading and writing. A practice report. Student Success, 9(3), 71-77. doi:https://doi.org/10.5204/ssj.v9i3.470

Kahu, E. R., Stephens, C., Zepke, N., \& Leach, L. (2014). Space and time to engage: Mature-aged distance students learn to fit study into their lives. International Journal of Lifelong Education, 33(4), 523-540. doi:https://doi.org/10.1080/02601370.2014.884177

Kaufman, P. (2014). The sociology of college students' identity formation. New Directions for Higher Education, 2014(166), 35-42. doi:https://doi.org/10.1002/he.20093

Kensington-Miller, B., Knewstubb, B., Longley, A., \& Gilbert, A. (2018). From invisible to SEEN: A conceptual framework for identifying, developing and evidencing unassessed graduate attributes. Higher Education Research \& Development, 37(7), 1439-1453. doi:https://doi.org/10.1080/07294360.2018.1483903

Kiernan, M. D., \& Hill, M. (2018). Framework analysis: A whole paradigm approach. Qualitative Research Journal, 18(3), 248-261. doi:https://doi.org/10.1108/QRJ-D-17$\underline{00008}$

King, A. (2010). The odd couple: Margaret Archer, Anthony Giddens and British social theory. The British Journal of Sociology, 61(s1), 253-260. doi:https://doi.org/10.1111/j.1468-4446.2009.01288.x

Klemenčič, M. (2018). Higher education in Europe in 2017 and open questions for 2018. The European Journal of Higher Education, 8(1), 1-4. doi:https://doi.org/10.1080/21568235.2013.871206

Krathwohl, D. R. (2009). Methods of educational and social science research: The logic of methods (3rd ed.). Long Grove, III.: Waveland.

Kuh, G. D., \& Bridges, B. K. (2010). What do university students learn? In International Encyclopedia of Education (pp. 479-484): Elsevier.

Lash, S. (1994). Reflexivity and its doubles: Structure, aesthetics, community. In U. Beck, A. Giddens, \& S. Lash (Eds.), Reflexive modernization: Politics, tradition and aesthetics in the modern social order (pp. 110-173). Cambridge, UK: Polity.

Leibowitz, B., Garraway, J., \& Farmer, J. (2015). Influence of the past on professional lives: A collective commentary. Mind, Culture \& Activity, 22(1), 23-36. doi:https://doi.org/10.1080/10749039.2014.979949

Lewis, J. (2007). Analysing qualitative longitudinal research in evaluations. Social Policy and Society, 6(4), 545-556. doi:https://doi.org/10.1017/S1474746407003880

Luckett, K., \& Luckett, T. (2009). The development of agency in first generation learners in higher education: A social realist analysis. Teaching in Higher Education, 14(5), 469481. doi:https://doi.org/10.1080/13562510903186618

Macfarlane, B. (2017). Freedom to learn: The threat to student academic freedom and why it needs to be reclaimed. Oxon, UK: Routledge.

Markus, H., \& Nurius, P. (1986). Possible selves. American Psychologist, 41(9), 954-969. doi:https://doi.org/10.1037/0003-066X.41.9.954

Massey University. (2014). Redesigning the BA: The core papers. Retrieved from Palmerston North, New Zealand:

Massey University. (2017a). Bachelor of Arts: Graduate profile. Retrieved from Palmerston North, New Zealand:

Massey University. (2017b). Code of ethical conduct for research, teaching and evaluations involving human participants: Revised code. Retrieved from http://www.massey.ac.nz/massey/fms/Human\%20Ethics/Documents/Revised\%20C ode.pdf?BBFDDEDDA9C2DBF2BAAC9C6951C58ED4

Massey University. (2017c). Massey University Annual Report 2017. Retrieved from Palmerston North, NZ: 
Matthews, B. (2017). "I wouldn't imagine having to go through all this and still be the same person. No way": Structure, reflexivity and international students. Journal of Research in International Education, 16(3), 265-278. doi:https://doi.org/10.1177/1475240917745611

Mayhew, M. J., Wolniak, G. C., Rockenbach, A. N., Bowman, N. A., \& Seifert, T. A. D. (2016). How college affects students. Volume 3, 21st century evidence that higher education works (Second edition.. ed.). San Francisco, CA: Jossey-Bass.

McGregor, L., Parker, K., LeBlanc, P., \& King, K. M. (2010). Using social exchange theory to guide successful study recruitment and retention. Nurse Researcher, 17(2), 7482. doi:https://doi.org/10.7748/nr2010.01.17.2.74.c7464

Mclnnis, C. (2004). Studies of student life: An overview. European Journal of Education, 39(4), 383-394.

McLeod, J., \& Thomson, R. (2009). Researching social change. Thousand Oaks, CA: Sage.

Mead, G. H. (1934). Mind, self \& society (C. W. Morris, D. R. Huebner, \& H. Joas Eds.). Chicago, IL: University of Chicago.

Menard, S. (2011). Longitudinal research. In M. S. Lewis-Beck, A. Brynam, \& T. F. Lio (Eds.), The Sage encyclopedia of social science research methods (Vol. 2, pp. 598-601). Thousand Oaks, CA: Sage.

Merriam, S. B. (1998). Qualitative research and case study applications in education. San Francisco, CA: Jossey-Bass.

Ministry of Education. (2014). Tertiary Education Strategy 2014 - 2019. Wellington, NZ Retrieved from http://www.education.govt.nz/further-education/policies-andstrategies/tertiary-education-strategy/

Ministry of Education. (2018). Education counts. Retrieved from https://www.educationcounts.govt.nz/statistics/tertiary education/participation

Ministry of Social Development. (2016). The Social Report 2016. Te pūrongo oranga tangata. Retrieved from New Zealand:

Moorfield, J. C. (2019). Te Aka online Māori dictionary. Retrieved from https://maoridictionary.co.nz/. Retrieved 30 October 2019, from Te Ipukarea, Pearson, Netvalue https://maoridictionary.co.nz/

Mrozowicki, A. (2011). Coping with social change: Life strategies of workers in Poland's new capitalism. Leuven, Belgium: Leuven University.

Mutch, A. (2004). Constraints on the internal conversation: Margaret Archer and the structural shaping of thought. Journal for the Theory of Social Behaviour, 34(4), 429445. doi:https://doi.org/10.1111/j.1468-5914.2004.00257.x

Nico, M., \& Caetano, A. (2015). Untying conceptual knots: The analytical limits of the concepts of de-standardisation and reflexivity. Sociology, 1-19. doi:https://doi.org/10.1177/0038038515604107

Ogilvie, A. D. (2017). Personal reflexivity and the construction of adolescent vocational identity. (Doctor of Philosophy). Queensland University of Technology, Australia,

Oyserman, D. (2004). Self-concept and identity. In M. B. Brewer \& M. Hewstone (Eds.), Self and social identity (pp. 5-24). Malden, MA: Blackwell.

Oyserman, D., Elmore, K., \& Smith, G. (2012). Self, self-concept, and identity. In M. R. Leary \& J. Price Tangney (Eds.), Handbook of self and identity (pp. 69-104). New York, NY: Guilford.

Pascarella, E. T., \& Terenzini, P. T. (2005). How college affects students (Vol. 2. A third decade of research). San Francisco: Jossey-Bass.

Poskitt, J., Rees, M., \& Suddaby, G. (2011). Engaging with university at a distance: The differences in levels of student engagement among extramural and campus-based students. In A. Radloff (Ed.), Student engagement in New Zealand's universities. Melbourne, AUS: Australian Council for Educational Research (ACER). 
Rainford, J. (2016). Making internal conversations public: Reflexivity of the connected doctoral researcher and its transmission beyond the walls of the academy. Journal of applied social theory, 1(1), 44-60. doi:https://doi.org/10.1177/2042753016672380

Rhoads, R. A., \& Szelenyi, K. (2011). Global citizenship and the university: Advancing social life and relations in an interdependent world. Stanford, CA: Stanford University

Ribisl, K. M., Walton, M. A., Mowray, C. T., Luke, D. A., Davidson II, W. S., \& Bootsmiller, B. J. (1996). Minimizing participant attrition in panel studies through the use of effective retention and tracking strategies: Review and recommendations. Education and Program Planning, 19(1), 1-25. doi:https://doi.org/10.1016/01497189(95)00037-2

Riessman, C. K. (1993). Narrative analysis. California, USA: Sage.

Ritchie, J., Lewis, J., McNaughton Nicholls, C., \& Ormston, R. (Eds.). (2014). Qualitative research practice: A guide for social science students and researchers. London, UK: Sage.

Ritchie, J., \& Spencer, L. (1994). Qualitative data analysis from applied policy research. In A. Bryman \& B. Burgess (Eds.), Analyzing qualitative data (pp. 173-194). London, UK: Routledge.

Rossiter, M. (2007). Possible selves and adult learning: Perspectives and potential. San Francisco, CA: Jossey-Bass.

Saldana, J. (2003). Longitudinal qualitative research: Analyzing change through time. Lanham, MD and Oxford, UK: Oxford.

Sayer, A. R. (2010). Reflexivity and the habitus. In M. S. Archer (Ed.), Conversations about reflexivity (pp. 108-122). New York, NY: Routledge.

Scholz, R. W., \& Tietje, O. (2002). Embedded case study methods: Integrating quantitative and qualitative knowledge. Thousand Oaks, CA: Sage.

Sharrock, W., \& Tsilipakos, L. (2013). A return to 'the inner' in social theory: Archer's 'internal conversation". In T. R. Racine \& K. L. Slaney (Eds.), A Wittgensteinian perspective on the use of conceptual analysis in psychology (pp. 195-213). Basingstoke: Palgrave McMillian.

Smith, C. (2010). What is a person?: Rethinking humaility, social life, and the moral good from the person up. Chicago, II: University of Chicago

Smith, G., Ottewill, R., Jubb, E., Sperling, E., \& Wyman, M. (2008). Teaching citizenship in higher education. European Political Science, 7(2), 135-143. Retrieved from http://ezproxy.massey.ac.nz/login?url=http://search.ebscohost.com/login.aspx?dire ct=true\&db=edsgao\&AN=edsgcl.179978247\&site=eds-live\&scope=site

Smith, J., \& Firth, J. (2011). Qualitative data analysis: Application of the framework approach. Nurse Researcher, 18(2), 52-62. doi:https://doi.org/10.7748/nr2011.01.18.2.52.c8284

Sparkes, A. C., \& Smith, B. (2015). Narrative analysis as an embodied engagement with the lives of others. In J. A. Holstein \& J. F. Gubrium (Eds.), Varieties of narrative analysis (pp. 53-74). Thousand Oaks, CA: Sage.

Spronken-Smith, R., Bond, C., McLean, A., Frielick, S., Jenkins, M., \& Marshall, S. (2013). How to engage with a graduate outcomes agenda: A guide for tertiary education institutions. Wellington, NZ: Ako Aotearoa: National Centre for Tertiary Teaching Excellence.

Stake, R. E. (1994). Case studies. In N. K. Denzin \& Y. Lincoln, S. (Eds.), Handbook of Qualitative Research. Thousand Oaks, CA: Sage.

Stake, R. E. (1995). The art of case study research. Thousand Oaks, CA: Sage.

Stets, J. E., \& Burke, P. J. (2012). A sociological approach to self and identity. In M. Leary \& J. Tangney (Eds.), Handbook of self and identity. New York, NY: Guilford. 
Stryker, S., \& Burke, P. J. (2000). The past, present, and future of an identity theory. Social Psychology Quarterly, 63(4), 284-297. Retrieved from http://ezproxy.massey.ac.nz/login?url=http://search.ebscohost.com/login.aspx?dire ct=true \&AuthType $=$ ip, cookie, url, uid\&db=ccm\&AN=2011942483\&site=ehostlive\&scope $=$ site

Sullivan, C. M., Rumptz, M. H., Campbell, R., Eby, K. K., \& Davidson II, W. S. (1996). Retaining participants in longitudinal community research: A comprehensive protocol. Journal of Applied Behavioral Science(3), 262. Retrieved from http://ezproxy.massey.ac.nz/login?url=http://search.ebscohost.com/login.aspx?dire $\mathrm{ct}=$ true\&db=edsgao\&AN=edsgcl.18740021\&site=eds-live\&scope=site

Sullivan, K. (1994). Bicultural education in Aotearoa/New Zealand: Establishing a Tauiwi side to the partnership. New Zealand Annual Review of Education, 3, 191-222.

Susen, S. (2016). The sociological challenge of reflexivity in Bourdieusian thought. In D. Robbins (Ed.), The Anthem companion to Pierre Bourdieu (pp. 49-94). Cambridge University: Anthem.

Thomson, R., \& Holland, J. (2003). Hindsight, foresight and insight: The challenges of longitudinal qualitative research. International Journal of Social Research Methodology, 6(3), 233-244. doi:https://doi.org/10.1080/1364557032000091833

Toledano, M. B., Smith, R. B., Brook, J. P., Douglass, M., \& Elliott, P. (2015). How to establish and follow up a large prospective cohort study in the 21st Century - Lessons from UK COSMOS. PLOS ONE, 27(2), 85-96. doi:https://doi.org/10.1371/journal.pone.0131521

Tolich, M. (2016). Qualitative ethics in practice. Walnut Creek, CA: Left Coast.

Tracy, S. J. (2013). Qualitative research methods : Collecting evidence, crafting analysis, communicating impact. Chichester, UK: Wiley-Blackwell.

Tsekeris, C., \& Lydaki, A. (2011). The micro-macro dilemma in sociology: Perplexities and perspectives. Sociologija, 53(1), 67-82. doi:https://doi.org/10.2298/SOC1101067T

UNESCO World Conference on Education. (1998). World declaration on higher education for the twenty-first century: Vision and action. Retrieved from www.unesco.org/education/educprog/wche/declaration eng.htm

University of Auckland. (2017). The New Zealand Attitudes and Values Study. Retrieved from https://www.psych.auckland.ac.nz/en/about/our-research/researchgroups/new-zealand-attitudes-and-values-study.html

Usher, R., Bryant, I., \& Johnston, R. (1997). Adult education and the post modern challenge: Learning beyond the limits. London, UK: Routledge.

Veugelers, W., de Groot, I., Llomovatte, S., \& Naidorf, J. (2017). Higher education, educational policy and citizenship development. Education and Society, 35(1), 2742.

Victoria University of Wellington. (2016). Human Ethics. Retrieved from https://www.wgtn.ac.nz/research/support/ethics/human-ethics

Vogl, S., Zartler, U., Schmidt, E.-M., \& Rieder, I. (2018). Developing an analytical framework for multiple perspective, qualitative longitudinal interviews (MPQL). International Journal of Social Research Methodology, 21(2), 177-190. doi:https://doi.org/10.1080/13645579.2017.1345149

Weidman, J. C., DeAngelo, L., \& Bethea, K. A. (2014). Understanding student identity from a socialization perspective. In New Directions for Higher Education [Article] (Vol. 166, pp. 43-51). doi:https://doi.org/10.1002/he.20094

Williams, K. F. (2012). Rethinking 'learning' in higher education: Viewing the student as social actor. Journal of Critical Realism, 11(3), 296-323. doi:https://doi.org/10.1558/icr.v11i3.296 
Wilson, J. (2005). Story: Society. In Te Ara - the Encyclopedia of New Zealand (2005 ed.). Wellington, NZ: Manatū Taonga Ministry for Culture and Heritage.

Yin, R. K. (2009). Case study research design and methods (4th ed. Vol. 5). Thousand Oaks, CA: Sage.

Zoom Video Communications. (2020). Zoom. Retrieved from https://zoom.us/ 


\section{Appendices}




\section{Appendix A: Recruitment phases and recruitment outcomes}

\begin{tabular}{|c|c|c|c|}
\hline Phase 1 & Type of contact & $\begin{array}{l}\text { Distribution and } \\
\text { Responses }\end{array}$ & Consideration \\
\hline $\begin{array}{l}\text { Pre-course } \\
\text { commencement } \\
\text { and Week } 2\end{array}$ & $\begin{array}{l}\text { Third Party Contact } \\
\text { (Bulk email to all } \\
\text { students from } \\
\text { external } \\
\text { administrator) }\end{array}$ & $\begin{array}{l}300 \text { emailed } \\
2 / 3 \text { responders } \\
\text { provide consent }\end{array}$ & $\begin{array}{l}\text { Students not ready for } \\
\text { study yet } \\
\text { High number of pre- } \\
\text { course \& institutional } \\
\text { emails } \\
\text { Spam filters respond to } \\
\text { bulk email } \\
\text { Email 'bounces' not } \\
\text { collected }\end{array}$ \\
\hline Course Week 4 & $\begin{array}{l}\text { Third Party Contact } \\
\text { (Grouped email to } \\
50 \text { students at a } \\
\text { time from external } \\
\text { administrator) }\end{array}$ & $\begin{array}{l}300 \text { emailed } \\
3 / 6 \text { responders } \\
\text { provide consent }\end{array}$ & $\begin{array}{l}\text { High number of pre- } \\
\text { course \& institutional } \\
\text { emails } \\
\text { Spam filters respond to } \\
\text { bulk email } \\
\text { Email 'bounces' not } \\
\text { collected }\end{array}$ \\
\hline $\begin{array}{l}\text { Course Week 4- } \\
5\end{array}$ & $\begin{array}{l}\text { Second Party } \\
\text { Contact } \\
\text { (Researcher } \\
\text { identity \& banner } \\
\text { established and } \\
\text { included) } \\
\text { Facebook page - } \\
\text { Citizenship } \\
\text { Research 2017-18 } \\
\text { created } \\
\text { Links to } \\
\text { independent } \\
\text { students Facebook } \\
\text { page established }\end{array}$ & & $\begin{array}{l}\text { Creation of researcher } \\
\text { and research identity to } \\
\text { authenticate research } \\
\text { Alternative contact } \\
\text { approaches within the } \\
\text { scope of ethics approval } \\
\text { identified } \\
\text { Ethics amended to allow } \\
\text { offer of gifts to } \\
\text { participants }\end{array}$ \\
\hline Course Week 7 & $\begin{array}{l}\text { First Party Contact } \\
\text { - Course } \\
\text { Coordinator } \\
\text { introduces } \\
\text { research, familiarity } \\
\text { with researcher } \\
\text { and independent } \\
\text { nature of research } \\
\text { in student } \\
\text { messages }\end{array}$ & $\begin{array}{l}300 \text { emailed in } \\
\text { batches of } 5 \\
9 / 11 \text { responders } \\
\text { provide consent }\end{array}$ & $\begin{array}{l}\text { Email includes Research } \\
\text { banner, and personal } \\
\text { introduction } \\
\text { Researcher collects } \\
\text { 'bounces' and re- send } \\
\text { individual emails } \\
\text { Repeat follow-up emails } \\
\text { sent }\end{array}$ \\
\hline
\end{tabular}




\begin{tabular}{|l|l|l|l|}
\hline & $\begin{array}{l}\text { Second party } \\
\text { contact email in } \\
\text { batches of 5 }\end{array}$ & \\
\hline $\begin{array}{l}\text { Course Weeks } \\
8-12\end{array}$ & $\begin{array}{l}\text { First Party Contact } \\
-\end{array}$ & $\begin{array}{l}\text { On-going email } \\
\text { exchanges } \\
\text { eialogue } \\
\text { established by } \\
\text { email. Thank-you } \\
\text { gift sent to } \\
\text { participants. } \\
\begin{array}{l}\text { Facebook posting } \\
\text { for more male } \\
\text { participants }\end{array}\end{array}$ & $\begin{array}{l}\text { Facebook 2/3 } \\
\text { responders } \\
\text { provide consent } \\
\text { first party contact }\end{array}$ \\
\hline
\end{tabular}




\section{Appendix B: Participant contexts and change over time}

\begin{tabular}{|c|c|c|c|c|c|}
\hline Participant & Gender & Age & Self-described Roles & $\begin{array}{l}\text { Work - study context } \\
\text { (Start of data collection) }\end{array}$ & $\begin{array}{c}\text { Change in work - study context } \\
\text { (End of data collection) }\end{array}$ \\
\hline 1 & M & $30 \mathrm{~s}$ & Public Service & $\begin{array}{l}\text { Full-time employment } \\
\text { Part-time study }\end{array}$ & $\begin{array}{l}\text { Work sabbatical } \\
\text { Full-time international study }\end{array}$ \\
\hline $\begin{array}{c}2 \\
\text { Māori }\end{array}$ & $\mathrm{F}$ & $40 \mathrm{~s}$ & Student Mother & Full-time study & Completed degree, looking for employment \\
\hline $\begin{array}{c}3 \\
\text { Māori }\end{array}$ & $\mathrm{F}$ & $30 s$ & $\begin{array}{l}\text { Living overseas, unable } \\
\text { to get employment }\end{array}$ & Full-time study & $\begin{array}{l}\text { New baby } \\
\text { Move to part-time study }\end{array}$ \\
\hline 4 & $\mathrm{~F}$ & 40s & $\begin{array}{l}\text { Student } \\
\text { Mother }\end{array}$ & Full-time study & Completed degree, looking for employment \\
\hline 5 & $\mathrm{~F}$ & $30 s$ & $\begin{array}{l}\text { Part-time student } \\
\text { Mother }\end{array}$ & Part-time study & $\begin{array}{l}\text { Relationship breakdown } \\
\text { Completed degree, looking for employment }\end{array}$ \\
\hline 6 & $\mathrm{~F}$ & $20 s$ & Full-time student & Full-time study & $\begin{array}{l}\text { Moved to a new city, new relationship } \\
\text { Completed degree, looking for employment }\end{array}$ \\
\hline 7 & $\mathrm{~F}$ & $30 s$ & $\begin{array}{l}\text { Full-time student } \\
\text { Mother }\end{array}$ & Full-time study & $\begin{array}{l}\text { Involved in children's school } \\
\text { Returned to full-time employment, part-time study }\end{array}$ \\
\hline 8 & $\mathrm{~F}$ & $30 \mathrm{~s}$ & $\begin{array}{l}\text { Full-time student } \\
\text { Mother } \\
\text { Community volunteer }\end{array}$ & $\begin{array}{l}\text { Full-time study } \\
\text { Shift to part time study and } \\
\text { employment }\end{array}$ & $\begin{array}{l}\text { Part-time employment in new job } \\
\text { Completed degree }\end{array}$ \\
\hline 9 & $\mathrm{~F}$ & $20 \mathrm{~s}$ & Full-time student & Full-time student & No change \\
\hline $\begin{array}{c}10 \\
\text { Māori }\end{array}$ & $\mathrm{F}$ & $30 \mathrm{~s}$ & $\begin{array}{l}\text { Work for charitable trust } \\
\text { Mother }\end{array}$ & $\begin{array}{l}\text { Full-time employment } \\
\text { Part-time student }\end{array}$ & $\begin{array}{l}\text { New baby } \\
\text { Part-time employment } \\
\text { Break from study }\end{array}$ \\
\hline $\begin{array}{l}11 \\
\text { European } \\
\text { immigrant }\end{array}$ & $\mathrm{F}$ & $20 s$ & $\begin{array}{l}\text { Full-time work for } \\
\text { political group } \\
\text { Study part-time }\end{array}$ & $\begin{array}{l}\text { Full-time employment, part- } \\
\text { time study }\end{array}$ & Job changes, break from study \\
\hline $\begin{array}{c}12 \\
\text { Māori }\end{array}$ & $\mathrm{F}$ & $30 s$ & $\begin{array}{l}\text { Public Service } \\
\text { Mother of } 3\end{array}$ & $\begin{array}{l}\text { Full-time employment, part- } \\
\text { time study }\end{array}$ & No change \\
\hline 13 & $\mathrm{~F}$ & $50 s$ & Public Service & $\begin{array}{l}\text { Full-time employment, part- } \\
\text { time study }\end{array}$ & No change \\
\hline $\begin{array}{l}14 \\
\text { European } \\
\text { immigrant }\end{array}$ & $\mathrm{F}$ & $40 s$ & & Part-time study & $\begin{array}{l}\text { Travel overseas } \\
\text { Part-time study }\end{array}$ \\
\hline
\end{tabular}




\begin{tabular}{|c|c|c|c|c|c|}
\hline 15 & $\mathrm{~F}$ & $20 s$ & $\begin{array}{l}\text { Single Mother and } \\
\text { Student }\end{array}$ & Part-time student. & No change \\
\hline $\begin{array}{c}16 \\
\text { Māori }\end{array}$ & M & $40 s$ & $\begin{array}{l}\text { Factory worker } \\
\text { Father }\end{array}$ & $\begin{array}{l}\text { Full-time employment, part- } \\
\text { time study }\end{array}$ & $\begin{array}{l}\text { Working longer hours } \\
\text { Part-time study }\end{array}$ \\
\hline $\begin{array}{c}17 \\
\text { Māori }\end{array}$ & $\mathrm{F}$ & $20 s$ & $\begin{array}{l}\text { Part-time Public Service } \\
\text { Student }\end{array}$ & $\begin{array}{l}\text { Part-time employment, part- } \\
\text { time study }\end{array}$ & $\begin{array}{l}\text { Moved overseas for employment } \\
\text { No longer studying }\end{array}$ \\
\hline 18 & $\mathrm{~F}$ & $50 \mathrm{~s}$ & Full-time Teacher & $\begin{array}{l}\text { Full-time employment, part- } \\
\text { time study }\end{array}$ & No change \\
\hline 19 & $\mathrm{~F}$ & $30 \mathrm{~s}$ & $\begin{array}{l}\text { Manufacturing work } \\
\text { Student }\end{array}$ & $\begin{array}{l}\text { Full-time employment, part- } \\
\text { time study }\end{array}$ & $\begin{array}{l}\text { Moved city } \\
\text { Full-time employment, part-time study }\end{array}$ \\
\hline $\begin{array}{c}20 \\
\text { Pacific person }\end{array}$ & M & $20 s$ & $\begin{array}{l}\text { Full-time work } \\
\text { Lives overseas }\end{array}$ & $\begin{array}{l}\text { Full-time employment, part- } \\
\text { time study }\end{array}$ & Withdrawn from study. \\
\hline 21 & $\mathrm{~F}$ & $<20$ & Student & Full-time student & Moved into flat. Full-time student \\
\hline 22 & M & $50 s$ & $\begin{array}{l}\text { Work in retail } \\
\text { Student }\end{array}$ & $\begin{array}{l}\text { Part-time employment, part- } \\
\text { time student }\end{array}$ & $\begin{array}{l}\text { Health interrupts study } \\
\text { Part-time employment, part-time student }\end{array}$ \\
\hline 23 & $\mathrm{~F}$ & $50 s$ & Mother & Part-time student & No change \\
\hline 24 & M & $20 s$ & $\begin{array}{l}\text { Full-time teacher } \\
\text { International sports }\end{array}$ & $\begin{array}{l}\text { Full-time employment, part- } \\
\text { time student }\end{array}$ & No change \\
\hline 25 & $\mathrm{~F}$ & $20 s$ & Part-time teacher & $\begin{array}{l}\text { Part-time employment, part- } \\
\text { time student }\end{array}$ & Move to full-time employment, part-time student \\
\hline $\begin{array}{c}26 \\
\text { Pacific person }\end{array}$ & $\mathrm{F}$ & $20 s$ & $\begin{array}{l}\text { Part-time student } \\
\text { Part-time work, Mother }\end{array}$ & $\begin{array}{l}\text { Part-time employment, part- } \\
\text { time student }\end{array}$ & $\begin{array}{l}\text { Change in degree, part-time student } \\
\text { Full-time employment one semester }\end{array}$ \\
\hline 27 & $\mathrm{~F}$ & $40 \mathrm{~s}$ & $\begin{array}{l}\text { Full-time public service, } \\
\text { part-time student } \\
\text { Mother }\end{array}$ & $\begin{array}{l}\text { Full-time employment, part- } \\
\text { time student }\end{array}$ & $\begin{array}{l}\text { Relationship breakdown } \\
\text { Full-time employment, part-time student }\end{array}$ \\
\hline 28 & M & $30 \mathrm{~s}$ & $\begin{array}{l}\text { Full-time in public } \\
\text { service, part-time student } \\
\text { Father }\end{array}$ & $\begin{array}{l}\text { Full-time employment, part- } \\
\text { time student. }\end{array}$ & $\begin{array}{l}\text { Moved locations, job change } \\
\text { Health issues Not studying }\end{array}$ \\
\hline 29 & $\mathrm{~F}$ & $50 s$ & $\begin{array}{l}\text { Business owner } \\
\text { Mother \& Grandmother }\end{array}$ & $\begin{array}{l}\text { Full-time employment, part- } \\
\text { time student }\end{array}$ & $\begin{array}{l}\text { Finished study } \\
\text { Completed diploma }\end{array}$ \\
\hline $\begin{array}{c}30 \\
\text { Māori } \\
\end{array}$ & $\mathrm{F}$ & $20 \mathrm{~s}$ & $\begin{array}{l}\text { Business owner } \\
\text { Mother }\end{array}$ & $\begin{array}{l}\text { Self-employed, part-time } \\
\text { student, part-time volunteer }\end{array}$ & $\begin{array}{l}\text { New baby, health issues } \\
\text { Not studying }\end{array}$ \\
\hline $\begin{array}{c}1 \\
\text { European } \\
\text { immigrant }\end{array}$ & $\mathrm{F}$ & $50 \mathrm{~s}$ & "Stay-at-home" Mother & $\begin{array}{l}\text { Part-time employment, part- } \\
\text { time student } \\
\text { Part-time volunteer }\end{array}$ & $\begin{array}{l}\text { Relationship breakdown } \\
\text { Full-time student, } \\
\text { Part-time volunteer }\end{array}$ \\
\hline
\end{tabular}




\section{Appendix C: Interview questions}

Q1. Who are you? Tell me about yourself?

Q2. What have been/are the influences on your life?

Q3. What the most important things to you?

Q4. Why are you studying?

Q5. How did you introduce yourself at the start of [the course]?

Q6. What did you like about [the course]?

Q7. Were there any ideas [in the course] that were challenging?

Q8. Was there anything in [the course] that changed your ideas of citizenship?

Q9. How would you describe yourself? As a citizen?

Q10. How would you introduce yourself now?

Q11. What do you think about this course being a core course?

Q13. How did you go about processing the ideas in the course?

Q14. What has changed for you since the last interview? 


\section{Appendix D: Additional questions for interviews: Archer's 10 mental activities of internal conversation}

The theorists that I am consulting in my research believe that we have internalised ways of looking at, deciding on, dealing with, new and old ideas presented to us, and in considering the things that are of value and important to us.

Some of the terms that Margaret Archer used are internal conversation, inner dialogue, inner conversation, inner deliberation, internalised considerations, contemplation, to consider inside one's head.

Q.1. Does this sound familiar to you?

Q.2. Describe to me how this would happen?

Archer has defined some 'mental activities', and I will provide a loose definition. Then I will ask for your thoughts about the activity like:

Q.3. Does this sound familiar to you?

Q.4. Can you please describe to me how this would happen?

Q.5. How often would you do this?

Q.6. When are you most likely to do this?

Q.7. Can you give me an example of when you do this?

\begin{tabular}{|l|l|}
\hline \multicolumn{1}{|c|}{$\begin{array}{c}\text { Archer's Mental } \\
\text { Activities: }\end{array}$} & \multicolumn{1}{c|}{ Definition } \\
\hline Planning & $\begin{array}{l}\text { Mental preparation in the act or process of making a plan, course of } \\
\text { action or strategy, in advance }\end{array}$ \\
\hline Rehearsing & $\begin{array}{l}\text { To practice through making a speech, relating facts or anticipating } \\
\text { actions, in private } \\
\text { Internalised preparation for an external exchange }\end{array}$ \\
\hline Mulling Over & $\begin{array}{l}\text { To think about carefully; to consider a particular object or thought; to } \\
\text { allow the mind to wander or ponder over unspecified thoughts }\end{array}$ \\
\hline Deciding & $\begin{array}{l}\text { Settling a question, uncertainty or dispute, leading to a final decision } \\
\text { on which to act }\end{array}$ \\
\hline
\end{tabular}




\begin{tabular}{|l|l|}
\hline Reliving & $\begin{array}{l}\text { To experience something again, with or without associated emotion. } \\
\text { Derived from thinking about your own history }\end{array}$ \\
\hline Prioritising & $\begin{array}{l}\text { To arrange, order and reject, organize or deal with something } \\
\text { according to its importance, urgency or other priority }\end{array}$ \\
\hline Imagining & $\begin{array}{l}\text { To form a mental image of (something not actually present to the } \\
\text { senses). To suppose, think or conjecture/imagine } \\
\text { Imagining extends possibility beyond existing boundaries }\end{array}$ \\
\hline Clarifying & $\begin{array}{l}\text { Sorting out what you think about some issue, problem or person, } \\
\text { performed internally for and subject to subject's own satisfaction } \\
\text { To make (an idea, statement, etc.) clear or intelligible; to be free from } \\
\text { ambiguity, confusion or uncertainty }\end{array}$ \\
\hline $\begin{array}{l}\text { Imaginary } \\
\text { Conversations }\end{array}$ & $\begin{array}{l}\text { Where you run through a conversation or discussion with people you } \\
\text { know or have heard of }\end{array}$ \\
\hline Budgeting & $\begin{array}{l}\text { To plan or allocate the distribution of resources - In terms of money, } \\
\text { time or effort }\end{array}$ \\
\hline
\end{tabular}


Appendix E: Framework analysis worked chart: Elsie

\begin{tabular}{|c|c|c|}
\hline Values and beliefs & Identity & Attitudes \\
\hline $\begin{array}{l}\text { T2 I love studying. } \\
\text { Community psychology, } \\
\text { Indigenous psychology. } \\
\text { Need to examine narratives } \\
\text { and counter-narratives in } \\
\text { ideologies \& society } \\
\text { Knowledge development and } \\
\text { interest personalised to be } \\
\text { consistent with values of } \\
\text { critique and examining } \\
\text { perspective (El200 I.130-5) } \\
\text { All interested in social } \\
\text { injustice and environmental } \\
\text { concerns, then } \\
\text { acknowledges personal } \\
\text { value not held by all in the } \\
\text { community }\end{array}$ & $\begin{array}{l}\text { T2 - Living context in } \\
\text { community - hippy/activist, } \\
\text { more than most people } \\
\text { Idealist person with BIG } \\
\text { ideas (El200 I.401-2) }\end{array}$ & $\begin{array}{l}\text { T2 Attitudinal struggle } \\
\text { between course requirement } \\
\text { and reality - the whole idea } \\
\text { of citizenship and how to fix } \\
\text { things in the world - which is } \\
\text { very much what was in the } \\
\text { course and about - } \\
\text { controlling capitalist resource } \\
\text { mongering in our own } \\
\text { western countries, rather } \\
\text { than going over and helping } \\
\text { people in other countries } \\
\text { (El200 I.113-7) } \\
\text { Attitude - full embodiment } \\
\text { more important than internal } \\
\text { thinking (El200 I.280-86) } \\
\text { using instinct } \\
\text { Mulling over done in body } \\
\text { not mind (EI200 I.316-18) } \\
\text { Mental imagery and mental } \\
\text { conversations if trying to } \\
\text { understand people, not } \\
\text { situations or knowledge } \\
\text { (El200 I.322-325) }\end{array}$ \\
\hline $\begin{array}{l}\text { T3 Value } \\
\text { - equal access to same } \\
\text { resources (El300 I.7) } \\
\text { Mental health resources \& } \\
\text { distance students). } \\
\text { - a goal of living in } \\
\text { communities is to share. } \\
\text { (El300 I.317). } \\
\text {..Underneath qualities of } \\
\text { connection and being able } \\
\text { to connect well with people } \\
\text { and about having the } \\
\text { capacity to connect with } \\
\text { people (El300 I 20-321) } \\
\text { - Intentional community is for } \\
\text { curious and engaged } \\
\text { optimists, this is the world } \\
\text { for those who are curious - } \\
\text { about how to make their } \\
\text { world a lot better (El300 } \\
\text { l.327-8) }\end{array}$ & $\begin{array}{l}\text { T3 Separated, solo parent } \\
\text { Interested in the learning - } \\
\text { Identity student } \\
\text { Living in wider community not } \\
\text { just immediate } \\
\text { Years of study have changed } \\
\text { thing. } \\
\text { Identity - recognition - like to } \\
\text { work with ideas, lot of skills, } \\
\text { able to be proactive in the } \\
\text { world (El300 I.350-54) }\end{array}$ & $\begin{array}{l}\text { T3 I think I would use some } \\
\text { of those ideas about framing } \\
\text { and story and how to } \\
\text { connect to people } \\
\text { emotionally through, to tell } \\
\text { stories, to bring core } \\
\text { messages through and not } \\
\text { just using facts and figures } \\
\text { and logic, but kind of using } \\
\text { that deeper connection } \\
\text { (El300 I.138-41). Use in } \\
\text { activism to create projects to } \\
\text { tell the stories of more } \\
\text { marginalised narratives and } \\
\text { that stuff (El300l.144-5) } \\
\text { Understanding of framing } \\
\text { has changed the way } * * * \\
\text { sees what is occurring in her } \\
\text { community meetings and } \\
\text { people's presentations. Then } \\
\text { in wider community in how } \\
\text { mental health is being } \\
\text { framed }\end{array}$ \\
\hline
\end{tabular}




\begin{tabular}{|c|c|c|}
\hline $\begin{array}{l}\text { Changes in values: Over } \\
\text { time the importance of this } \\
\text { shifts as focus of discussion } \\
\text { becomes more external, next } \\
\text { steps }\end{array}$ & $\begin{array}{l}\text { Changes in identity - I } \\
\text { guess my sense of myself as } \\
\text { a person that can work with, } \\
\text { that likes to work with ideas } \\
\text { and in the world of ideas - } \\
\text { reading, writing, } \\
\text { conceptualizing, integrating } \\
\text { and particularly thinking } \\
\text { about taking that and pulling } \\
\text { together, I have got a lot of } \\
\text { skills in that area [laughs] } \\
\text { and I'm the sort of person } \\
\text { that is able to do that and } \\
\text { that is has grown. Yeah. And } \\
\text { I do feel more able to use } \\
\text { those skills in the world to - to } \\
\text { be more proactive in the } \\
\text { world (El300 I. 350-354) }\end{array}$ & $\begin{array}{l}\text { Changes in attitude of } \\
\text { activism as something in the } \\
\text { past which didn't appear to } \\
\text { work to something that can } \\
\text { be done using the resources } \\
\text { gathered in the interim and in } \\
\text { future research. }\end{array}$ \\
\hline
\end{tabular}




\section{Appendix F: Research ethics approval}

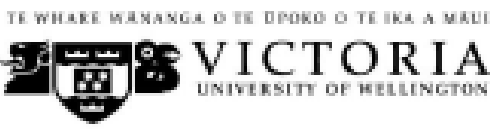

MEMORANDUM

\begin{tabular}{|c|c|}
\hline TO & Unda Rowan \\
\hline COPYTO & Dr Stephanle Doyle \\
\hline FROM & AProf Susan Corbett, Convener, Human Ethics Committee \\
\hline DATE & 15 December 2016 \\
\hline PAGES & 1 \\
\hline SUENECT & $\begin{array}{l}\text { Ethics Approval: } 22478 \\
\text { The social construction of ctizenship through a dedcated } \\
\text { programme of studty in higher education: Internal conversaton and } \\
\text { socletal influences }\end{array}$ \\
\hline
\end{tabular}

Thank you for your appilcation for ethical approval, which has now been considered by the Standing Commintee of the Human Etrics Committee.

Your apolication has been approved from the above date and this approval continues until 1 ivarch 2019 . If your data collection is not completed by this date you should apply to the Human Ethics Committee for an extension to this approval.

Best wshes wth the research.

Kind reqards

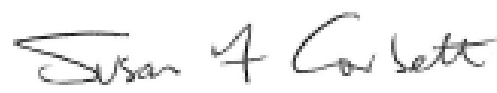

Susan Corbett

Convener, Vctorla Unlversity Human Ethics Committee 


\section{Appendix G: Memo prepared from interview one transcript for interview two}

\section{Memo from Ch100 interview transcript Ch200 interview}

For the next interview I have three objectives:

1. To find out about experience of learning about citizenship in [the course]?

2. To determine any changes in circumstances.

3. To clarify some comments made in transcript 1 relating to priorities.

Background: Ch is a life-long learner with a number of qualifications and experiences of [work]. This involves a lot of contact and training in working with diverse cultures. Brought up with strong Christian values and community focus. Core values - "work, can't expect others to prop you up, if you want something you work for it, parents help where they can" (Ch100 l.82-84).

Lives in a smaller community and involved in community activities. Is the first port of call in emergency due to recognition of [work] expertise.

Important - family, work and work-life balance.

Social justice important. "I have a good sense of social justice I suppose" (Ch100 I.65)

Aware of issues of power.

Discusses course ideas with family and siblings, other [work people] - a wide pool of people. Provides examples of critiquing ideas with family.

Discussion is in the present with reflection back to provide examples, nothing on future thinking.

Liked the course. $\mathrm{E}^{* * *}$ interactions, flow and the way "you felt that you were building a relationship with the tutors rather than them there saying you have to learn $x, y, z \ldots$

"(Ch100 I. 110 -114). Course makes you think. "The course makes you think about how you've been brought up ..." (Ch100 I 71 -75) to be active in your community

Processes - reading - mostly while running, talks about mulling over. One of the ${ }^{* * \star}$ will read an article and they will discuss it.

Talked about core values from parents and core values from [work]

Q. What are these core values and their influence on $C^{* * *}$ ?

Q. Need to collect more in-depth information on citizenship and ideas of what citizenship is about.

Q. Need to collect more information on priorities and concerns. 
